

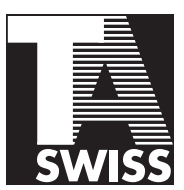

Brunngasse 36

$\mathrm{CH}-3011$ Bern

TA-SWISS 69/2019

Sarab Fässler, Regina Aebi-Müller,

Franziska Müller, Vera Hertig, Alexander Lueger,

Christian Kind, Andreas Balthasar

\section{Social Freezing - Kinderwunsch auf Eis}




\section{Bibliografische Information der Deutschen Nationalbibliothek}

Die Deutsche Nationalbibliothek verzeichnet diese Publikation in der Deutschen Nationalbibliografie; detaillierte bibliografische Daten sind im Internet über http://dnb.d-nb.de abrufbar.

Dieses Werk einschliesslich aller seiner Teile ist urheberrechtlich geschützt. Jede Verwertung ausserhalb der engen Grenzen des Urheberrechtsgesetzes ist ohne Zustimmung des Verlages unzulässig und strafbar. Das gilt insbesondere für Vervielfältigungen, Übersetzungen, Mikroverfilmungen und die Einspeicherung und Verarbeitung in elektronischen Systemen.

This work is licensed under creative commons license CC BY-NC-ND $2.5 \mathrm{CH}$.

SOME RIGHTS RESEERVED

\section{Zitiervorschlag}

Fässler, S., Aebi-Müller, R., Müller, F., Hertig, V., Lueger, A., Kind, Ch., Balthasar, A. (2019). Social Freezing - Kinderwunsch auf Eis. In TA-SWISS Publikationsreihe (Hrsg.): TA 69/2019. Zürich: vdf

\section{Danksagung}

Das Projektteam möchte allen Personen danken, die sich Zeit für unsere Befragungen und Interviews genommen haben. Sie haben wichtige Grundlagen für die Studie geliefert. Auch TA-SWISS und allen Mitgliedern der Begleitgruppen sind wir für die konstruktive Zusammenarbeit, die anregenden Diskussionen und die wertvollen Rückmeldungen zu Dank verpflichtet.

Coverabbildungen:

(C) Links: iStock.com/Tsuji

(C) Rechts: iStock.com/igohugo

\section{() 2019 vdf Hochschulverlag AG an der ETH Zürich}

ISBN 978-3-7281-3962-7 (Printausgabe)

Download open access:

ISBN 978-3-7281-3963-4 / DOI 10.3218/3963-4

www.vdf.ethz.ch

verlag@vdf.ethz.ch 


\section{Inhaltsverzeichnis}

Zusammenfassung 11

Executive Summary $\quad 19$

Résumé $\quad 27$

Sintesi $\quad 36$

1. Einleitung 45

$\begin{array}{lll}1.1 & \text { Forschungsfragen } & 46\end{array}$

1.2 Methodisches Vorgehen 48

2. Technologie 59

2.1 Beschreibung der Technologie $\quad 59$

2.2 Risiken 63

2.3 Erfolgsaussichten $\quad 80$

2.4 Anwendung der Technologie in der Schweiz 88

3. Aktuelle Nachfrage nach Social Freezing 99

3.1 Internationale Studien zur Nachfrage in anderen Ländern 99

3.2 Empirische Befunde zur Nachfrage in der Schweiz 110

4. Potenzielles Interesse am Social Freezing 117

4.1 Internationale Studien zum potenziellen Interesse $\begin{array}{ll}\text { am Social Freezing } & 117\end{array}$

$\begin{array}{ll}\text { 4.2 Empirische Befunde zum potenziellen Interesse } & 122 \\ \text { am Social Freezing in der Schweiz }\end{array}$

5. Diskussion des Social Freezing in der internationalen Literatur 135

5.1 Diskussion der ethischen Dimension des Social Freezing 135

5.2 Diskussion der gesellschaftlichen Dimension des Social Freezing 148

5.3 Diskussion der finanziellen Dimension des Social Freezing 156 
6. Diskussion des Social Freezing in den Schweizer Medien

6.1 Diskussion der ethischen Dimension in den Schweizer Medien 162

6.2 Diskussion der gesellschaftlichen Dimension des Social Freezing in den Schweizer Medien

6.3 Diskussion der finanziellen Dimension des Social Freezing in den Schweizer Medien

6.4 Diskussion der rechtlichen Dimension des Social Freezing in den Schweizer Medien

7. Meinungstypen zum Social Freezing bei Schweizer Akteuren

7.1 Beschreibung der drei Meinungstypen zum Social Freezing 174

7.2 Konsens und Kompromisse im politischen Meinungsdiskurs 180

$\begin{array}{lll}\text { 8. } & \text { Rechtsvergleichung } & 185\end{array}$

$\begin{array}{lll}8.1 & \text { Übersicht } & 185\end{array}$

$\begin{array}{lll}8.2 & \text { Schweiz } & 185\end{array}$

$\begin{array}{lll}8.3 \text { Österreich } & 188\end{array}$

8.4 Frankreich 190

8.5 Italien 200

8.6 Deutschland 204

$\begin{array}{lll}8.7 & \text { Belgien } & 206\end{array}$

$\begin{array}{lll}8.8 \text { Spanien } & 210\end{array}$

8.9 Tschechische Republik 213

$\begin{array}{ll}\text { 9. Schlussfolgerungen } & 217\end{array}$

9.1 Beantwortung der Forschungsfragen 217

$\begin{array}{lll}9.2 & \text { Fazit } & 249\end{array}$

9.3 Empfehlungen 254

A1 Anhang: Literaturverzeichnis 257

A2 Anhang: Medienartikel 269

A3 Anhang: Q-Methode 275

A4 Anhang: Rechtsvergleichung 283

A4.1 Österreich 283

$\begin{array}{ll}\text { A4.2. Frankreich } & 285\end{array}$

A4.3, Italien 287 
$\begin{array}{lll}\text { A4.4 Deutschland } & 288\end{array}$

$\begin{array}{ll}\text { A4.5 Belgien } & 289\end{array}$

A4.6 Spanien und Tschechische Republik 291

Mitglieder der Begleitgruppe $\quad 293$

Mitglieder der Projektgruppe 294

Projektmanagement TA-SWISS 294 


\section{Abbildungsverzeichnis}

Abbildung 1: Likert-Skala eines erzwungenen Verteilungsverfahrens von 51 Statements .......................................................... 56

Abbildung 2: Die drei Phasen des Social Freezing .................................. 60

Abbildung 3: Übersicht über die medizinischen Risiken für Frau und Kind in Zusammenhang mit Social Freezing .................. 64

Abbildung 4: Beratungsinhalte und vertragliche Regelungen.................... 91

Abbildung 5: Alterslimite für die Eizellentnahme...................................... 94

Abbildung 6: Änderung der gesetzlichen Rahmenbedingungen ............... 96

Abbildung 7: Kenntnis über Social Freezing nach soziodemografischen

Variablen, in Prozent .................................................... 123

Abbildung 8: Beeinflussung der Einstellung zum Social Freezing durch Informationen, in Prozent.......................................... 126

Abbildung 9: Interesse am Social Freezing: Könnten Sie sich grundsätzlich vorstellen, Ihre Eizellen vorsorglich einfrieren zu lassen?

Abbildung 10: Wichtigster Beweggrund für Social Freezing nach Alterskategorie, in Prozent............................................. 128

Abbildung 11: Alter für die Einfrierung der Eizellen ................................. 130 


\section{Tabellenverzeichnis}

Tabelle 1: Anzahl und Anteil kinderloser Frauen nach Altersgruppen (Vergleich Bevölkerung mit Stichprobe) ................................... 51

Tabelle 2: $\quad$ Erfolgsaussichten des Social Freezing ....................................... 84

Tabelle 3: Übersicht über die Kryokonservierung von Eizellen in 17 europäischen Ländern .................................................. 100

Tabelle 4: $\quad$ Szenarien zur potenziellen Entwicklung der Nachfrage nach Social Freezing .......................................................... 133

Tabelle 5: Übersicht über die Rechtslage in acht Ländern ...................... 245

Tabelle 6: Statements zu Social Freezing mit Faktorenwerten (Q-sort values) für die Faktoren........................................... 277 


\section{Zusammenfassung}

Unter Social Freezing wird das vorsorgliche Einfrieren eigener Eizellen bezeichnet, um einen Kinderwunsch auch noch in höherem Alter zu verwirklichen. Dabei nehmen Frauen ohne medizinische Notwendigkeit fortpflanzungsmedizinische Verfahren in Anspruch, die eigentlich für die Behandlung von Unfruchtbarkeit entwickelt wurden. Dies erlaubt innen, ihre Schwangerschaft zeitlich nach hinten zu versetzen, um beispielsweise einen passenden Partner zu finden oder die Karriere voranzutreiben. Zum Zeitpunkt des Einfrierens der Eizellen sind entsprechend soziale und nicht medizinische Gründe ausschlaggebend für die Nutzung der Technologie.

Die medizinische Technologie des Social Freezing beruht auf drei zeitlich abgegrenzten Phasen. In der ersten Phase erfolgt die Hormonbehandlung zur Stimulation der Eierstöcke (ovarielle Stimulation). Danach werden diese über die Scheide mit einer Nadel punktiert und die Eizellen abgesaugt. Die zweite Phase umfasst die Kryokonservierung der Eizellen. Darunter wird das Einfrieren von Eizellen in flüssigem Stickstoff bei minus $196 \mathrm{Grad}$ verstanden. In der dritten Phase werden die Eizellen aufgetaut und mit den Spermien des Wunschvaters in der Glasschale (in vitro) befruchtet. Die erste und die dritte Phase entsprechen einer klassischen In-vitro-Befruchtung, wie sie mittlerweile seit 40 Jahren praktiziert wird. Das Einfrieren der Eizellen wird hingegen erst seit gut zehn Jahren in grösserem Stil praktiziert und hat einen breiten Einsatz des Social Freezing erst ermöglicht. Ausschlaggebend für diese Entwicklung war ein neues Verfahren zur Kryokonservierung der Eizellen. Mit der Vitrifikation werden die Eizellen schockgefroren, wodurch die Bildung von zerstörerischen Eiskristallen in den Eizellen vermieden wird. Die Schwangerschaftsraten bei der (in vitro) Befruchtung von kryokonservierten Eizellen unterscheiden sich dadurch kaum von den Raten, die mit frischen Eizellen erreicht werden.

Die Technologie verspricht, den Druck der biologischen Uhr auf die Frauen zu reduzieren. Gleichzeitig wirft Social Freezing Fragen zu medizinischen Risiken auf, löst ethische Bedenken bezüglich des Kindeswohls aus und untergräbt möglicherweise gesellschaftliche Bestrebungen zur Vereinbarkeit von Beruf und Familie. 
Um diese Chancen und Risiken der Technologie aus einer umfassenden Perspektive zu beleuchten, wurden in der vorliegenden Studie verschiedene methodische Zugänge kombiniert. Für eine Auslegeordnung der medizinischen Chancen und Risiken sowie der ethischen, gesellschaftlichen, finanziellen und rechtlichen Folgen des Social Freezing wurde eine Literaturanalyse realisiert. Um das Angebot und die Nutzung des Social Freezing in der Schweiz besser zu verstehen, wurde eine Onlinebefragung bei allen Fertilitätskliniken in der Schweiz durchgeführt. Ergänzend wurden mit einzelnen Reproduktionsmedizinern und Frauen, die ihre Eizellen einfrieren liessen, explorative Hintergrundinterviews geführt. Um das Interesse am Social Freezing in der Schweiz besser zu verstehen und das Potenzial dieser Technologie abzuschätzen, wurden 408 kinderlose Frauen im Alter zwischen 25 und 39 Jahren online befragt. Die Einstellungen zum Thema Social Freezing in der Schweizer Gesellschaft wurden einerseits über eine Medienanalyse aufgearbeitet. Andererseits wurden 23 Akteure interviewt, die alle politischen Parteien sowie für das Thema Social Freezing relevanten gesellschaftlichen Gruppierungen repräsentieren. Schliesslich wurde die Gesetzgebung der Schweiz mit derjenigen in Österreich, Frankreich, Italien, Deutschland, Belgien, Spanien und der Tschechischen Republik verglichen. Nachfolgend werden die wichtigsten Erkenntnisse aus der Studie präsentiert.

\section{Chancen und Risiken der Technologie noch wenig erforscht}

Die ovarielle Stimulation und die Befruchtung der Eizellen in vitro werden seit mittlerweile 40 Jahren praktiziert. Die Kryokonservierung von Eizellen ist hingegen eine neuere Erscheinung. Entsprechend dünn ist die Datenlage zu den gesundheitlichen Risiken der Kryokonservierung der Eizellen für die Kinder. Besser erforscht sind die Risiken, die mit den beiden anderen Phasen der Technologie verbunden sind. Allerdings werden diese in den Diskussionen zum Social Freezing häufig ausgeblendet. Die ovarielle Stimulation und die Befruchtung der Eizellen in vitro sind mit Risiken wie Schwangerschaftsvergiftungen, Frühgeburtlichkeit, niedrigem Geburtsgewicht und einer leicht erhöhten Fehlbildungsrate verbunden. Weiter bestehen Hinweise, dass diese Verfahren zu mehr Krebserkrankungen im Kindesalter führen und die kognitive Entwicklung der Kinder beeinträchtigen. Schliesslich begünstigen die reproduktionsmedizinischen Verfahren das Auftreten von Veränderungen am Herz-Kreislauf-System, die ursächlich mit chronischen Erkrankungen und Todesfällen im Erwachse- 
nenalter in Zusammenhang stehen. Das Ausmass und die Bedeutung dieser Forschungsergebnisse müssen allerdings noch in weiteren, gross angelegten epidemiologischen Studien geklärt werden.

Schätzungen zur Wahrscheinlichkeit, durch Social Freezing ein Kind zur Welt zu bringen, beruhen sowohl international als auch in der Schweiz auf unterschiedlichen Grundlagen. Bei Frauen unter 35 Jahren wird die Chance auf ein Kind mit 60 bis 80 Prozent angegeben, wenn 15 bis 20 Eizellen eingefroren worden sind. Sowohl im Ausland als auch in der Schweiz lassen Frauen allerdings aus Kostengründen oder wegen Nebenwirkungen der Behandlung teilweise deutlich weniger Eizellen einfrieren. Da bisher nur wenige Frauen auf ihre eingefrorenen Eizellen zurückgegriffen haben, kann die Erfolgsaussicht dieser Technologie noch nicht zuverlässig beurteilt werden.

\section{Geringe und vorwiegend durch fehlenden Partner motivierte Nachfrage}

Aktuell ist die Nachfrage nach Social Freezing gering. Jährlich lassen geschätzt 300 bis 400 Frauen ihre Eizellen in der Schweiz kryokonservieren. Im Ausland sind diese Frauen tendenziell über 35 Jahre alt, alleinstehend und gut ausgebildet. Auch in der Schweiz dürfte das Profil der Frauen ähnlich sein.

Nur rund die Hälfte der befragten kinderlosen Frauen zwischen 25 und 39 Jahren kennen die Technologie, die Nachfrage steigt allerdings kontinuierlich. In der Schweiz sind 34 Prozent der befragten kinderlosen Frauen grundsätzlich am Social Freezing interessiert. Sieben Prozent dieser Frauen zeigen ein konkretes Interesse an dem Verfahren. Das Potenzial des Social Freezing dürfte sich somit auf zwischen 2000 und 10'000 Frauen belaufen, die jährlich ihre Eizellen kryokonservieren lassen.

Deutlich weniger Frauen dürften ihre eingefrorenen Eizellen in vitro befruchten lassen. Gemäss internationalen Studien haben bisher lediglich 10 Prozent der Frauen auf ihre kryokonservierten Eizellen zurückgegriffen. Rund ein Drittel der Frauen mit eingefrorenen Eizellen erachten es als sehr wahrscheinlich, dass sie ihre Eizellen in vitro befruchten lassen werden.

Als Motivation für die Kryokonservierung der Eizellen wird von den befragten Frauen nicht die Karriere, sondern der fehlende Partner genannt. Gesucht wer- 
den Partner, welche dem Bild des «neuen Vaters» entsprechen. Diese Väter nehmen gemeinsam mit den Müttern die Verantwortung für die Kindererziehung wahr und möchten sich intensiv in der Kinderbetreuung engagieren.

Dämpfend auf das Interesse am Social Freezing wirken sich insbesondere Informationen zu den Kosten und den Risiken für Frau und Kind aus. Aufgrund der raschen technologischen und sozialen Veränderungen in diesem Bereich sind Nachfragevoraussagen allerdings mit grosser Unsicherheit behaftet.

Grundlagen für einen informierten Entscheid aus ethischer Sicht zentral, jedoch nicht in jedem Fall gegeben

Im Zentrum der ethischen Diskussion über Social Freezing stehen die Bedeutung eines informierten Entscheides für die Technologie sowie die Berücksichtigung des Kindeswohls. Alle 23 gesellschaftlichen Akteure, die im Rahmen der Studie interviewt wurden, erachten eine ausführliche Beratung der Frauen als sehr wichtige Grundlage für einen informierten Entscheid. Eine umfassende Aufklärung ist gerade bei Wahleingriffen ohne medizinische Indikation grundsätzlich von grosser Bedeutung. Allerdings besteht bei einer Mehrheit der interviewten gesellschaftlichen Akteure die Befürchtung, dass die Anbieter aufgrund von kommerziellen Interessen die Entscheidung der Frauen beeinflussen. Eine Minderheit der Interviewten schliesst eine solche Beeinflussung durch die Anbieter aus. Die vorliegende Studie liefert Hinweise, dass einzelne Frauen nicht umfassend über die Risiken für Frau und Kind informiert waren.

\section{Das fortgeschrittene Alter der Mutter im Zentrum der ethischen Diskussion zum Kindeswohl}

In der Literatur wird vor allem das Alter der Mutter bei der Befruchtung der Eizellen in vitro und die damit verbundenen medizinischen Risiken für das Kind betont. Auch die 23 interviewten Schweizer Akteure sprechen sich in erster Linie aufgrund der medizinischen Risiken für eine Altersgrenze bei der In-vitroBefruchtung aus. Andere Begründungen für eine Altersgrenze wie die Chancengleichheit der Geschlechter im Zugang zur Reproduktionsmedizin, die Ressourcen älterer Mütter in der Kindererziehung sowie veränderte Familienbilder 
und Generationenbeziehungen spielen eine untergeordnete Rolle. Eine Mehrheit der interviewten Schweizer Akteure befürwortet eine fixe Altersgrenze.

Einfluss des Social Freezing auf die gesellschaftlichen Bemühungen zur Vereinbarkeit von Beruf und Familie im Zentrum der gesellschaftlichen Diskussionen

In der gesellschaftlichen Diskussion steht der Einfluss des Social Freezing auf die Vereinbarkeit von Beruf und Familie im Mittelpunkt. Bei den interviewten gesellschaftlichen Akteuren sowie in den Schweizer Medien überwiegt dabei die Skepsis. Es wird befürchtet, dass Social Freezing anstelle von arbeitsrechtlichen, politischen oder gesellschaftlichen Massnahmen zur Vereinbarkeit von Beruf und Familie tritt und sich negativ auf die Chancengleichheit der Geschlechter auswirkt. Ausschlaggebend für die Inanspruchnahme des Social Freezing sind gemäss Aussagen von den befragten Frauen und Reproduktionsmedizinern jedoch nicht primär Karriereüberlegungen, sondern vielmehr der fehlende passende Partner. Vor diesem Hintergrund wäre eine umfassende und systematische Reflexion über das Zusammenspiel von Social Freezing und den Familiengründungsabsichten nicht nur von Frauen, sondern auch von Männern dringlich. Die Auswirkungen einer breiten Anwendung des Social Freezing auf die öffentliche Gesundheit, insbesondere die Häufigkeit von nicht übertragbaren Krankheiten, sind weder international noch in der Schweiz Gegenstand der Diskussion.

Weder Arbeitgeber noch soziale Krankenversicherung kommen für die Kostenübernahme infrage

Sowohl international als auch bei den interviewten gesellschaftlichen Akteuren in der Schweiz besteht weitgehend ein Konsens, dass weder der Arbeitgeber noch die soziale Krankenversicherung einen Beitrag an die Kryokonservierung von Eizellen leisten sollten. Dafür müssten primär die Frauen selbst aufkommen. Im Zentrum steht das Argument, dass Sozial Freezing aus sozialen und nicht aus medizinischen Gründen eingesetzt wird. Auch eine Beteiligung an den Kosten der In-vitro-Befruchtung im Rahmen des Social Freezing wird von den befragten Akteuren abgelehnt. Nur eine Minderheit könnte sich eine solche 
Kostenbeteiligung im Sinne der Chancengleichheit zugunsten von weniger gut verdienenden Frauen vorstellen.

\section{Altersgrenze statt Aufbewahrungsfrist im Fokus der rechtlichen Regelung des Social Freezing}

Eine Rechtsvergleichung des Social Freezing in sieben anderen europäischen Ländern zeigt zwei wesentliche Unterschiede: Einerseits sehen die meisten Länder eine Altersgrenze für die In-vitro-Befruchtung der kryokonservierten Eizellen vor. Andererseits gibt es mit Ausnahme von Belgien kein Land, das eine Aufbewahrungsfrist für die Eizellen vorsieht. Im Gegensatz zur Schweiz kann diese Lagerungsdauer in Belgien allerdings im Ermessen der Fertilitätskliniken jederzeit verlängert werden. Während die Aufbewahrungsfrist medizinisch nicht begründet werden kann, sprechen nicht nur medizinische, sondern auch ethische, gesellschaftliche und rechtliche Überlegungen für eine Altersgrenze bei der In-vitro-Befruchtung.

Kein Verbot des Social Freezing, aber noch viele Bedenken und Unsicherheiten

Insgesamt zeigt sich: Beim Social Freezing besteht ein weites Meinungsspektrum. Es handelt sich um ein sehr persönliches Thema, das stark von individuellen Faktoren wie Alter, Geschlecht und der eigenen Situation bezüglich Kinderwunsch geprägt wird. Ein Verbot des Social Freezing stellt für die Mehrheit der 23 interviewten gesellschaftlichen Akteure keine Option dar, dennoch gibt es viele Bedenken und Unsicherheiten rund um die Anwendung dieser Technik. Die Meinungsbildung in der Schweiz scheint noch nicht sehr weit fortgeschritten zu sein und es besteht weiterhin ein Diskussionsbedarf.

Gestützt auf die in der Studie gewonnenen Erkenntnisse hat die Projektgruppe zusammen mit der Begleitgruppe sechs Empfehlungen formuliert:

\section{Empfehlung 1: Zurückhaltende Anwendung des Social Freezing}

Durch Social Freezing werden fortpflanzungsmedizinische Technologien bei gesunden Frauen angewendet - ohne medizinische Notwendigkeit. Dies bringt eine erhöhte ethische Verantwortung mit sich, insbesondere im Hinblick auf die 
Risiken für die Kinder und die Auswirkungen auf die öffentliche Gesundheit. Zu den Folgen der Kryokonservierung liegen erst wenige Daten vor. Zudem weisen neuere Studienergebnisse darauf hin, dass die reproduktionsmedizinischen Verfahren sich negativ auf das Herz-Kreislauf-System betroffener Kinder auswirken. Vor einer breiten Anwendung des Social Freezing sind deshalb verlässlichere Informationen über die medizinischen, sozialen und psychologischen Risiken für die betroffenen Kinder notwendig. Bis dahin sollte die Technologie nur zurückhaltend genutzt und angewendet werden.

\section{Empfehlung 2: Förderung der Forschung zu Risiken des Social Freezing}

Um verlässliche Informationen zu medizinischen und anderen Risiken der Fortpflanzungsmedizin zu erhalten, sollte die Forschung zum Social Freezing gefördert werden, beispielsweise im Rahmen eines nationalen Forschungsprogramms (NFP) des Schweizerischen Nationalfonds zur Fortpflanzungsmedizin. In einem solchen Programm könnten neben medizinischen auch ethische, soziale und rechtliche Aspekte des Social Freezing untersucht werden. Weiter sind internationale Kooperationen zur Erforschung der Langzeitfolgen wichtig, damit genügend grosse Zahlen von Studienteilnehmern erreicht und aussagekräftige Ergebnisse erzielt werden können.

\section{Empfehlung 3: Die Erfassung der Daten zum Social Freezing gewährleisten}

Um die Nachfrage nach Social Freezing sowie dessen Folgen besser einschätzen zu können, sind verlässliche Daten seitens der Kliniken und Labore notwendig. Es sollten jährlich Informationen zur Anzahl und zum Alter der Frauen vorliegen, die ihre Eizellen aus sozialen Gründen kryokonservieren lassen. Weiter sollte erfasst werden, wie viele Frauen die eingefrorenen Eizellen in vitro befruchten lassen und in welchem Alter sie dies tun. Schliesslich sollten Daten zu den Schwangerschafts- und Geburtenraten im Rahmen des Social Freezing gesammelt und ausgewertet werden.

\section{Empfehlung 4: Einen informierten Entscheid sicherstellen}

Interessierte Frauen sollten umfassende Informationen zum medizinischen Verfahren, zu den Risiken für Frau und Kind, den Erfolgsaussichten, den Kosten 
und den rechtlichen Rahmenbedingungen erhalten. Es gibt Hinweise, dass interessierte Frauen heute von Ärzten und Kliniken teilweise ungenügend informiert werden. Idealerweise werden Frauen unabhängig und umfassend zu sämtlichen Aspekten des Social Freezing beraten. Prüfenswert wäre, ein Informationsangebot zum Social Freezing bei den kantonalen Fachstellen für Familienplanung und sexuelle Gesundheit anzusiedeln.

Empfehlung 5: Aufhebung der Aufbewahrungsfrist zugunsten einer Altersgrenze

Die Frist für die Aufbewahrung der eingefrorenen Eizellen von maximal zehn Jahren sollte zugunsten einer Altersgrenze aufgehoben werden. Eine Schwangerschaft in fortgeschrittenem Alter birgt medizinische Risiken mit gesundheitlichen Folgen für Mutter und Kind. Zudem ist eine Elternschaft in fortgschrittenem Alter mit sozialen Risiken verbunden. Im Adoptionsrecht wurde ein maximaler Altersunterschied zwischen Adoptivkind und Adoptiveltern von 45 Jahren vorgesehen. Um eine Wertungskongruenz zwischen Adoptions- und Fortpflanzungsrecht sicherzustellen, sollte eine maximale Altersgrenze von 45 Jahren für Schwangerschaften nach einer In-vitro-Befruchtung festgelegt werden. Mit der Einführung einer Altersgrenze würden die eingefrorenen Eizellen nicht mehr nach Ablauf der Aufbewahrungsfrist, sondern nach Erreichen der Altersgrenze vernichtet.

Empfehlung 6: Keine Finanzierung des Social Freezing durch die Krankenkassen

Die Kosten für das Einfrieren von Eizellen sollten nicht von den Krankenversicherungen übernommen werden. Krankenkassen sind primär für die Kostenfolgen von Krankheiten oder deren Prävention zuständig. Eine individuelle Vorsorge für den eventuellen Fall, dass bei altersbedingter Unfruchtbarkeit noch ein Kinderwunsch besteht, kann jedoch nicht als Krankheitsprävention bezeichnet werden. 


\section{Executive Summary}

Social freezing refers to the precautionary freezing of a woman's own eggs in order to realise a desire for children at an older age. Women thereby take advantage of reproductive medical procedures without medical necessity, although these procedures were actually developed for the treatment of infertility. This allows women to postpone pregnancy in order to find a suitable partner, for example, or to advance a career. When freezing the eggs, the corresponding social and non-medical reasons are the decisive factors for the use of this technology.

The medical technology of social freezing is based on three distinct phases. In the first phase, hormonal treatment is used to stimulate the ovaries (ovarian stimulation). Then, they are punctured by a needle drawn through the vagina and the eggs are sucked out. The second phase involves cryopreservation of the eggs. This means freezing the eggs in liquid nitrogen at minus 196 degrees. In the third phase, the eggs are thawed and fertilised (in vitro) with the sperm of the desired father in a glass petri dish. The first and the third phases correspond to classic in vitro fertilisation, as has been practised now for 40 years. Egg freezing, on the other hand, has only been practised for a good ten years on a larger scale and has just paved the way to the wider use of social freezing. A new process for the cryopreservation of eggs was crucial for this development. The eggs are snap-frozen with vitrification, thus avoiding the formation of destructive ice crystals in the eggs. Pregnancy rates in the (in vitro) fertilisation of cryopreserved eggs hardly differ from the rates achieved with fresh eggs.

The technology promises to reduce the pressure of the biological clock for women. At the same time, social freezing raises questions about medical risks, triggers ethical concerns about the child's well-being, and possibly undermines social efforts to achieve compatibility of career and family.

Various methodological approaches are combined in the present study using a comprehensive perspective in order to shed light on the opportunities and risks created by this technology. A literature review was performed to analyse the medical benefits and risks as well as the ethical, social, financial and legal consequences of social freezing. An online survey was conducted at all the fertility 
clinics in Switzerland in order to better understand the offer and use of social freezing in Switzerland. In addition, exploratory background interviews were carried out with individual reproductive health professionals as well as with women who had had their eggs frozen. In order to better understand the interest in social freezing in Switzerland and to assess the potential of this technology, 408 childless women between the ages of 25 and 39 were interviewed online. Firstly, the attitudes on the issues of social freezing in Swiss society were appraised via a media analysis. Then, 23 actors - representing all the political parties as well as the civic groups relevant for the subject of social freezing were interviewed. Finally, Swiss legislation was compared to that of Austria, France, Italy, Germany, Belgium, Spain and the Czech Republic. The main findings from the study are presented below.

There is still little research on the benefits and risks of the technology

Ovarian stimulation and the fertilisation of eggs in vitro have been practised for 40 years now. The cryopreservation of eggs, however, is a recent phenomenon. Correspondingly, the data is sparse on the health risks of egg cryopreservation for the children. The risks that are associated with the other two phases of the technology are better understood. However, these are often disregarded in the discussions on social freezing. Ovarian stimulation and in vitro fertilisation of eggs are associated with risks such as pre-eclampsia, prematurity, low birth weight and a slightly increased malformation rate. There is also evidence that these procedures lead to more childhood cancers and impairments in children's cognitive development. Finally, the reproductive medical procedures facilitate the onset of changes in the cardiovascular system, which are causally linked to chronic diseases and deaths in adulthood. Nevertheless, the extent and significance of these research results remain to be clarified in other, large-scale epidemiological studies.

The assessments on the probability of giving birth to a child with the help of social freezing are based on different foundations both internationally and in Switzerland. In women under 35 years of age, the chances of a child are indicated as being 60 to 80 percent when 15 to 20 eggs have been frozen. Both abroad and in Switzerland, however, women sometimes freeze significantly fewer eggs due to cost reasons or because of the treatment side effects. So far, 
because only a few women have resorted to using their frozen eggs, the success prospects of this technology cannot be reliably assessed yet.

Demand is low and primarily due to lack of partner

Currently, the demand for social freezing is low. An estimated 300 to 400 women have their eggs cryopreserved in Switzerland every year. Abroad, these women tend to be over 35 years of age, single and well educated. The profile of the women in Switzerland is likewise similar.

Only about half of the interviewed childless women between the ages of 25 and 39 know of the technology; nonetheless, the demand is rising steadily. In Switzerland, 34 percent of the childless women interviewed are generally interested in social freezing. Seven percent of these women show a concrete interest in the procedure. The potential of social freezing may likely amount to between 2,000 and 10,000 women who would have their eggs cryopreserved each year.

Significantly fewer women are likely to have their frozen eggs fertilised in vitro. According to international studies, so far only ten percent of women have used their cryopreserved eggs. Around one-third of women with frozen eggs consider it as very likely that they would have their eggs fertilised in vitro.

The women interviewed indicate that it is the lack of a partner, rather than the advancement of their careers, which serves as the motivating factor for egg cryopreservation. Women are looking for partners who correspond to the image of the 'new father'. These fathers take responsibility for raising children together with the mothers and wish to be intensively involved in the childcare.

Information on the costs and the risks for women and children in particular has a dampening effect on the interest in social freezing. Due to the rapid technological and social changes in this field, demand forecasting is admittedly fraught with great uncertainty. 
Basis for an informed decision is central from an ethical point of view, but not always given

The importance of an informed decision in terms of the technology as well as the consideration of the child's well-being are central to the ethical discussion about social freezing. All 23 social actors interviewed in the study were of the opinion that an in-depth consultation with the women is a crucial basis for making an informed decision. In principle, comprehensive information on this subject is of great importance, especially in the case of elective interventions without medical indication. However, a majority of the social actors interviewed fear that providers would influence women's choices on account of commercial interests. A minority of the interviewees exclude such influence by the providers. The present study provides evidence that individual women were not fully informed about the risks to women and children.

The advanced age of the mother at the centre of the ethical discussion on the child's well-being

The literature predominantly emphasises the age of the mother during the fertilisation of the eggs in vitro as well as the associated medical risks to the child. The 23 interviewed Swiss actors first and foremost speak out in favour of an age limit for in vitro fertilisation due to medical risks. Other justifications for an age limit such as equal opportunities for the sexes in accessing reproductive medicine and the resources of older mothers in child-rearing, as well as the changing perception of family and intergenerational relationships, play a subordinate role. A majority of the Swiss actors interviewed advocate a fixed age limit.

Influence of social freezing on social efforts to achieve compatibility between career and family at the centre of the social debate

The influence of social freezing on achieving compatibility between career and family life is at the heart of the social debate. Scepticism prevails among the social actors interviewed as well as in the Swiss media. It is feared that social freezing will replace employment laws, or political or social measures aimed at achieving compatibility between career and family, and will have a negative 
impact on equal opportunities for the sexes. However, according to statements made by women and reproductive health professionals, the decisive factor for the use of social freezing is the lack of a suitable partner rather than career deliberations. In this light, a comprehensive and systematic reflection on the interplay of social freezing and the intentions of establishing a family would be deemed urgent not only by women, but also by men. The effects of widespread use of social freezing on public health - the incidence of non-communicable diseases in particular - are not being discussed either internationally or in Switzerland.

Neither employer nor social health insurance comes into question in terms of the cost coverage

There is broad consensus both internationally and among the interviewed social actors in Switzerland that neither the employer nor the social health insurance should contribute to the cryopreservation of eggs. Essentially, the women themselves should pay for it. The central argument is that social freezing is used for social rather than medical reasons. A contribution to the cost of in vitro fertilisation in the context of social freezing is likewise rejected by the actors interviewed. Only a minority could envision such cost sharing in the sense of equal opportunities in favour of less well-paid women.

Age limit instead of retention period as the focus of the legal regulations on social freezing

A comparison of social freezing laws with seven other European countries shows two major differences: firstly, most countries have an age limit for the in vitro fertilisation of cryopreserved eggs. And secondly, with the exception of Belgium, there is no country which has a retention period for the eggs. Unlike with Switzerland, however, in Belgium this storage period may be extended at the discretion of the fertility clinics at any time. There is no medical justification for the retention period; however, not only medical but also ethical, social and legal considerations call for an age limit for in-vitro fertilisation. 
No prohibition of social freezing but still many concerns and uncertainties

Overall, we see that there is a broad range of opinions on social freezing. It is a very personal issue which is heavily influenced by individual factors such as age, gender and one's own situation regarding the desire to have children. A prohibition of social freezing is not an option for the majority of the 23 social actors interviewed; nevertheless, there are many concerns and uncertainties surrounding the application of this technique. Opinion-formation in Switzerland does not seem to be very advanced yet and there is still a need for discussion.

Based on the findings obtained in the study, the project group formulated six recommendations together with the supervisory group.

\section{Recommendation 1: Restrained application of social freezing}

Social freezing uses reproductive technologies for healthy women without any medical necessity. This brings increased ethical responsibilities, in particular with regard to the risks to children and the impact on public health. There is only limited data on the consequences of cryopreservation. Moreover, recent study results indicate that reproductive medical procedures have a negative effect on the cardiovascular system of affected children. More reliable information on the medical, social and psychological risks for the affected children is therefore necessary prior to a wider application of social freezing. Until then, the technology should be used and applied with restraint.

\section{Recommendation 2: Promote research on the risks of social freezing}

In order to obtain reliable information on medical and other risks of reproductive medicine, research on social freezing should be encouraged, for example as part of a National Research Programme (NRP) of the Swiss National Science Foundation on reproductive medicine. In addition to the medical aspects, the ethical, social and legal aspects of social freezing could also be examined through such a programme. Furthermore, international collaboration on the research of long-term consequences is important in order to acquire a sufficiently large number of study participants so as to attain meaningful results. 
Recommendation 3: Ensure the collection of data on social freezing

In order to better assess the demand for social freezing and its consequences, reliable data is required from clinics and laboratories. There should be annual information on the number and the ages of the women who have their eggs cryopreserved for social reasons. The number of women who have the frozen eggs fertilised in vitro - and at what age they do so - should also be recorded. Finally, data on pregnancy and birth rates should be collected and evaluated within the framework of social freezing.

Recommendation 4: Verify that the decision is an informed one

Interested women should receive comprehensive information on the medical procedure, the risks for the woman and the child, the prospects of success, the costs and the legal framework conditions. At present, there is evidence that interested women are sometimes inadequately informed by doctors and clinics. Ideally, women should be given independent and comprehensive advice on all aspects of social freezing. It may be worthwhile to implement a proper offer of information on social freezing in the specialist cantonal offices for family planning and sexual health.

Recommendation 5: Cancellation of the retention period in favour of an age limit

The deadline of the retention of frozen eggs for a maximum of ten years should be lifted in favour of an age limit. A pregnancy at an advanced age carries medical risks with health-related consequences for both mother and child. Additionally, parenthood at an advanced age is associated with social risks. The adoption law provides for a maximum age difference of 45 years between adoptive children and adoptive parents. In order to ensure congruency between adoption and reproductive rights, a maximum age limit of 45 years should be set for pregnancies after the in vitro fertilisation. With the introduction of an age limit, the frozen egg would no longer be destroyed after expiry of the retention period, but rather after reaching the age limit. 
Recommendation 6: No funding of social freezing by the health insurance companies

The cost of freezing eggs should not be covered by health insurance. Health insurance funds are primarily responsible for the cost implications of diseases or their prevention. An individual provision for the possible eventuality of agerelated infertility and the continued desire for a child, however, cannot be designated as disease prevention. 


\section{Résumé}

La congélation préventive d'ovocytes ou "Social Freezing » fait référence à la congélation de ses propres ovocytes en prévision de réaliser ultérieurement un désir d'enfant même à un âge avancé. Certaines femmes qui n'ont pas besoin de traitement médical, recourent à des méthodes de procréation médicalement assistée qui ont initialement été développées pour traiter l'infertilité. Cela leur permet de reporter leur grossesse dans le temps pour, par exemple, trouver le bon partenaire ou pour poursuivre leur carrière professionnelle. Ce sont des raisons sociales et non médicales qui ont été déterminantes pour le recours à la technologie au moment de la congélation des ovocytes.

La technologie médicale de la congélation préventive d'ovocytes repose sur trois phases distinctes. Pendant la première phase, un traitement hormonal est utilisé pour stimuler les ovaires (stimulation ovarienne). Une aiguille est ensuite insérée dans le vagin pour atteindre les ovaires, et les ovocytes sont aspirés. La deuxième phase implique la cryoconservation des ovocytes. Cette procédure implique la congélation des ovocytes dans de l'azote liquide à moins 196 degrés Celsius. Lors de la troisième phase, les ovocytes sont décongelés et fécondés avec le sperme du père choisi par la femme dans une coupelle en verre (in vitro). La première et la troisième phase correspondent aux étapes d'une fécondation in vitro classique, telle qu'elle est pratiquée depuis 40 ans maintenant. En revanche, la congélation des ovocytes n'est pratiquée à grande échelle que depuis une dizaine d'années, et elle a permis une généralisation de la congélation préventive des ovocytes. Un nouveau procédé de cryoconservation des ovocytes a été déterminant pour le développement de la congélation préventive des ovocytes. La vitrification permet de surgeler les ovocytes, ce qui évite la formation de cristaux de glace destructeurs dans les ovocytes. Les taux de grossesse de la fécondation (in vitro) des ovocytes cryo-conservés ne sont donc guère différents des taux de grossesse obtenus avec des ovocytes frais.

La technique promet de réduire la pression de l'horloge biologique qui pèse sur les femmes. Dans le même temps, la congélation préventive d'ovocytes soulève des questions sur les risques médicaux. Elle suscite des inquiétudes d'ordre éthique quant au bien-être de l'enfant, et elle peut nuire aux efforts que la société fait pour concilier la vie professionnelle et la vie familiale. 
Différentes approches méthodologiques sont présentées ensemble dans la présente étude pour faire la lumière sur les possibilités et les risques de cette technologie d'un point de vue plus général. Une analyse bibliographique a été réalisée pour analyser les possibilités et les risques médicaux, ainsi que les conséquences éthiques, sociales, financières et juridiques de la congélation préventive d'ovocytes. Afin de mieux comprendre l'offre et le recours à la congélation préventive d'ovocytes en Suisse, une étude en ligne a été menée dans toutes les cliniques suisses qui sont spécialisées dans le traitement de l'infertilité. Des entretiens exploratoires ont également été effectués avec quelques médecins spécialistes de la procréation médicalement assistée et des femmes, qui ont fait congeler leurs ovocytes. Pour mieux comprendre l'intérêt de la congélation préventive d'ovocytes en Suisse et estimer le potentiel de cette technologie médicale, 408 femmes sans enfant et âgées entre 25 et 39 ans ont répondu à un questionnaire en ligne. L'attitude de la société suisse envers la congélation préventive d'ovocytes a été étudiée, d'une part, par une analyse des médias et, d'autre part, par une enquête menée auprès de 23 parties prenantes qui représentent tous les partis politiques, ainsi que des groupes sociaux concernés par la congélation préventive d'ovocytes. Enfin, la législation suisse a été comparée aux législations de l'Autriche, de la France, de l'Italie, de l'Allemagne, de la Belgique, de l'Espagne et de la République tchèque. Les principales conclusions de l'étude sont présentées ci-dessous.

\section{Les possibilités et les risques de la technologie sont encore peu étudiés}

La stimulation ovarienne et la fécondation in vitro des ovocytes sont désormais pratiquées depuis 40 ans. Cependant, la cryoconservation des ovocytes est une technologie récente. Les données disponibles sur les risques pour la santé liés à la cryoconservation des ovocytes chez les enfants conçus de cette façon sont donc très limitées. Les risques associés aux deux autres phases de la technologie sont mieux compris. Néanmoins, ils sont souvent occultés dans les discussions sur la congélation préventive d'ovocytes. La stimulation ovarienne et la fécondation in vitro des ovocytes sont associées à des risques comme p. ex. l'éclampsie, les accouchements prématurés, le faible poids de naissance et une légère augmentation du taux de malformations. II y a également des signes montrant que ces procédures causent davantage de cancers infantiles et qu'elles nuisent au développement cognitif des enfants. Enfin, les procédures de la procréation médicalement assistée favorisent le développement d'anoma- 
lies du système cardio-vasculaire qui ont un lien de causalité avec les maladies chroniques et les décès à l'âge adulte. Cependant, l'importance et la signification de ces résultats de recherche doivent encore être clarifiées par la réalisation d'études épidémiologiques approfondies et à plus grande échelle.

Les estimations de la probabilité de donner naissance à un enfant à l'aide de la congélation préventive d'ovocytes reposent sur plusieurs bases, tant en Suisse qu'à l'étranger. Chez les femmes âgées de moins de 35 ans, la probabilité d'avoir un enfant serait de 60 à $80 \%$ lorsque 15 à 20 ovocytes ont été congelés. Cependant, en Suisse comme à l'étranger, le nombre d'ovocytes congelés par femme est parfois beaucoup plus faible pour des raisons de coût ou à cause des effets secondaires du traitement. Étant donné que seules quelques femmes ont déjà eu recours à leurs ovocytes congelés, le succès de cette technique ne peut pas encore être évalué de manière fiable.

Les demandes sont faibles et surtout motivées par le fait de ne pas avoir trouvé de partenaire

La demande pour la congélation préventive d'ovocytes est actuellement faible. Le nombre des femmes qui cryo-conservent leurs ovocytes en Suisse, est compris entre 300 et 400 par an. À l'étranger, ces femmes ont généralement plus de 35 ans, et elles sont célibataires et d'un non niveau de formation. Le profil de ces femmes est probablement similaire en Suisse.

Parmi les femmes sans enfant et âgées entre 25 à 39 ans interrogées, seule la moitié d'entre elles connaissent cette technique, mais la demande est en constante augmentation. En Suisse, $34 \%$ des femmes nullipares interrogées sont en principe intéressées par la congélation préventive d'ovocytes. Sept pour cent de ces femmes manifestent un intérêt concret pour la procédure. Chaque année, la congélation préventive d'ovocytes concernerait potentiellement entre 2000 et 10000 femmes qui vont faire cryo-conserver leurs ovocytes.

Mais le nombre de femmes qui procèdent à la fécondation in vitro leurs ovocytes congelés, est nettement plus faible. Selon les études internationales, seules $10 \%$ des femmes ont utilisé leurs ovocytes cryo-conservés. Environ un tiers des femmes ayant fait congeler leurs ovocytes estiment que leurs ovocytes seront très probablement fécondés in vitro. 
Pour justifier la cryoconservation de leurs ovocytes, les femmes interrogées n'ont pas cité leur carrière professionnelle, mais plutôt l'absence de partenaire. Elles recherchent des partenaires qui correspondent à l'image du "nouveau père ». Ces pères sont responsables de l'éducation des enfants conjointement avec les mères, et ils souhaitent participer activement à la garde des enfants.

Les informations sur les coûts et les risques pour les femmes et les enfants à naître ont un effet particulièrement dissuasif sur l'intérêt des femmes pour la congélation préventive d'ovocytes. En raison des développements technologiques et des changements sociaux rapides dans ce domaine, les prévisions de la demande restent empreintes d'une grande incertitude.

Les informations sont fondamentales pour prendre une décision éclairée d'un point de vue éthique, mais elles ne sont pas toujours transmises

L'importance de la décision éclairée par rapport à la technologie, ainsi que la prise en compte du bien-être de l'enfant à naître sont au centre du débat sur la congélation préventive d'ovocytes. L'ensemble des 23 parties prenantes de la société interrogées dans le cadre de l'étude considèrent que les entretiens approfondis avec les femmes constituent un fondement très important pour la prise d'une décision éclairée. II est essentiel de leur donner des informations complètes, en particulier dans le cas d'une intervention élective qui est réalisée sans raison médicale. Cependant, la majorité des parties prenantes interrogées craignent que les prestataires de la congélation préventive d'ovocytes influencent les choix des femmes pour des intérêts commerciaux. Une minorité des personnes interrogées exclut cette influence de la part de ces prestataires. La présente étude montre que certaines femmes n'étaient pas pleinement informées des risques qu'elles encouraient pour elles-mêmes et leurs enfants.

L'âge avancé de la mère est au centre du débat éthique sur le bien-être de l'enfant à naître

La littérature spécialisée met particulièrement l'accent sur l'âge de la mère au moment de la fécondation in vitro des ovocytes et sur les risques médicaux associés pour l'enfant à naître. Les 23 parties prenantes suisses interrogées sont également favorables à l'établissement d'une limite d'âge pour la féconda- 
tion in vitro, principalement en raison des risques médicaux encourus. Les autres raisons pour l'établissement d'une limite d'âge, telles que l'égalité des sexes dans l'accès à la procréation médicalement assistée, les meilleures ressources des mères plus âgées pour élever leurs enfants, et la modification de la représentation de la famille et des relations entre les générations, jouent un rôle mineur. Une majorité des parties prenantes suisses interrogées préconisent d'établir une limite d'âge fixe.

L'impact de la congélation préventive d'ovocytes sur les efforts que la société fait pour concilier la vie professionnelle et la vie familiale, est au cœur du débat social.

L'impact de la congélation préventive d'ovocytes sur la compatibilité entre la vie professionnelle et la vie familiale est au cœur du débat social. Le scepticisme prévaut au sein des parties prenantes de la société interrogées, ainsi que dans les médias suisses. Ils ont peur que la congélation préventive d'ovocytes ne remplace les mesures prises dans les domaines professionnels, politiques ou sociaux pour concilier la vie professionnelle et la vie familiale, et que cela nuise à l'égalité des sexes. Toutefois, selon les femmes et les médecins spécialistes de la procréation médicalement assistée qui ont été interrogés, le principal facteur qui détermine le recours à la congélation préventive d'ovocytes, n'est pas le développement de la carrière professionnelle, mais bien le fait de ne pas avoir trouvé le bon partenaire. Dans ce contexte, il est urgent d'avoir une réflexion globale et systématique sur les interactions entre la congélation préventive d'ovocytes et l'intention de fonder une famille, non seulement pour les femmes, mais également pour les hommes. Les effets du recours généralisé à la congélation préventive d'ovocytes sur la santé publique, en particulier la prévalence des maladies non transmissibles, ne sont discutés ni à l'échelle internationale, ni en Suisse.

L'employeur et l'assurance maladie ne prennent pas en charge les coûts générés

II existe un large consensus parmi les parties prenantes de la société interrogées, tant en Suisse qu'à l'étranger, selon lequel ni les employeurs ni l'assurance maladie sociale ne devraient contribuer au coût de la cryoconservation 
des ovocytes. Les femmes devraient payer elles-mêmes le coût pour la congélation préventive de leurs ovocytes. L'argument majeur est que la congélation préventive d'ovocytes est utilisée pour des raisons sociales, et non pour des raisons médicales. La participation aux coûts de la fécondation in vitro dans le cadre de la congélation préventive d'ovocytes est également rejetée par les parties prenantes interrogées. Seule une minorité des personnes interrogées pourraient envisager un tel partage des coûts dans un souci d'égalité des chances en faveur des femmes à revenus modestes.

Une limite d'âge au lieu de la durée de conservation est le point de mire de la réglementation de la congélation préventive d'ovocytes

Une étude comparative sur la conservation préventive d'ovocytes dans sept autres pays européens montre deux différences majeures: premièrement, la plupart des pays prévoient une limite d'âge pour la fécondation in vitro des ovocytes cryo-conservés et, deuxièmement, aucun pays ne prévoit de durée de conservation des ovocytes à l'exception de la Belgique. Contrairement à la Suisse, cette durée de conservation peut être prolongée à tout moment en Belgique, à la discrétion des cliniques spécialisées dans le traitement de l'infertilité. Bien que la durée de conservation ne puisse pas être justifiée médicalement, des considérations non seulement d'ordre médical, mais également éthique, social et juridique plaident en faveur de l'établissement d'une limite d'âge pour la fécondation in vitro.

La congélation préventive des ovocytes n'est pas interdite, mais il reste toujours de nombreuses préoccupations et incertitudes

Dans l'ensemble, cela montre qu'il existe un large spectre d'opinions sur la congélation préventive d'ovocytes. C'est un sujet très personnel qui est fortement influencé par des facteurs personnels tels que l'âge, le sexe et la situation de chacun par rapport au désir d'enfant. L'interdiction de la congélation préventive d'ovocytes n'est pas une option envisageable pour la majorité des 23 parties prenantes interrogées. Cependant, de nombreuses préoccupations et incertitudes entourent l'utilisation de cette technologie. La formation de l'opinion en Suisse ne semble pas suffisamment avancée et un débat sur le sujet reste nécessaire. 
En se basant sur les conclusions de l'étude, le groupe de projet et le groupe d'accompagnement ont formulé six recommandations :

Recommandation 1 : Application mesurée de la congélation préventive d'ovocytes

La congélation préventive d'ovocytes utilise des techniques de la procréation médicalement assistée chez des femmes en bonne santé - sans nécessité médicale. Cela implique une responsabilité éthique accrue, notamment en ce qui concerne les risques pour les enfants concernés et l'impact sur la santé publique. II n'existe encore que peu de données sur les conséquences de la cryoconservation. En outre, les résultats des études récentes suggèrent que les procédures de la procréation médicalement assistée ont un impact négatif sur le système cardio-vasculaire des enfants conçus avec ces méthodes. Avant de généraliser davantage la congélation préventive d'ovocytes, il est donc nécessaire de disposer d'informations plus fiables sur les risques médicaux, sociaux et psychologiques pour les enfants concernés. Pour le moment, la technique devrait être utilisée et appliquée avec modération.

Recommandation 2 : Encourager la recherche sur les risques de la congélation préventive d'ovocytes

Pour obtenir des informations fiables sur les risques médicaux et les autres risques liés à la procréation médicalement assistée, il est important d'encourager les recherches sur la congélation préventive d'ovocytes, par exemple dans le cadre d'un programme de recherche national (PRN) du Fonds national suisse sur la procréation médicalement assistée. Dans un tel programme, il serait possible d'étudier non seulement les aspects médicaux, mais également les conséquences éthiques, sociales et juridiques de la congélation préventive d'ovocytes. Les collaborations internationales seront également importantes pour étudier les conséquences à long terme, afin de pouvoir atteindre un nombre suffisant de participants aux études et d'obtenir des résultats significatifs. 
Recommandation 3 : Assurer la collecte de données sur la congélation préventive d'ovocytes

Il est nécessaire de disposer de données fiables provenant des cliniques et des laboratoires pour mieux évaluer la demande pour la congélation préventive d'ovocytes et ses conséquences. Les données annuelles informant sur le nombre et l'âge des femmes qui ont cryo-conservé leurs ovocytes pour des raisons sociales, doivent être rendues disponibles. II convient également de consigner le nombre de femmes ayant procédé à la fécondation in vitro de leurs ovocytes et l'âge qu'elles avaient à ce moment-là. Enfin, les données sur les taux de grossesse et de natalité suite à la congélation préventive d'ovocytes devraient être collectées et évaluées.

\section{Recommandation 4 : Garantir la prise de décision éclairée}

Les femmes intéressées par la congélation préventive d'ovocytes devraient recevoir des informations complètes sur la procédure médicale, les risques pour elles-mêmes et les enfants à naître, les probabilités de réussite, les coûts engendrés et le cadre légal. Certaines données montrent que les femmes intéressées sont parfois mal informées par les médecins et les cliniques. Dans l'idéal, les femmes devraient recevoir des conseils indépendants et des informations complètes sur tous les aspects de la congélation préventive d'ovocytes. II serait donc intéressant d'examiner la possibilité de mettre à disposition des informations sur la congélation préventive d'ovocytes dans les centres de planning familial et de santé sexuelle des cantons.

Recommandation 5 : Suppression de la durée de conservation au profit de l'établissement d'une limite d'âge

La durée maximale de dix ans pour la conservation des ovocytes congelés devrait être levée au profit d'une limite d'âge. Mener une grossesse à un âge avancé comporte des risques médicaux, avec des conséquences sur la santé de la mère et de l'enfant. En outre, la parentalité à un âge avancé est associée à des risques sociaux. Le droit en matière d'adoption prévoit une différence d'âge maximale entre l'enfant adopté et les parents adoptifs de 45 ans. Afin d'harmoniser les droits en matière d'adoption et les droits en matière de pro- 
création médicalement assistée, il convient de fixer une limite d'âge maximale de 45 ans pour les grossesses qui sont le fruit d'une fécondation in vitro. Avec la mise en place d'une limite d'âge, les ovocytes congelés ne seraient plus détruits après l'expiration de la durée de conservation, mais après avoir atteint la limite d'âge.

Recommandation 6 : Aucun financement de la congélation préventive d'ovocytes par les caisses d'assurance maladie

Le coût de la congélation des ovocytes ne devrait pas être pris en charge par une assurance maladie. Les caisses d'assurance maladie sont, avant tout, responsables des conséquences financières des maladies ou de leur prévention. Un acte préventif individuel qui est réalisé en prévision de la réalisation d'un désir d'enfant malgré l'infertilité liée à l'âge avancé, ne peut pas être considéré comme un acte de prévention des maladies. 


\section{Sintesi}

Per social freezing s'intende il congelamento precauzionale di propri oociti per poter realizzare il desiderio di procreare anche in età avanzata. A tal fine, le donne ricorrono a un metodo di procreazione con assistenza medica sviluppato in realtà per curare la sterilità, senza che ciò sia necessario dal punto di vista medico. Così facendo possono rimandare la gravidanza ad esempio in attesa di trovare un partner adatto o per dedicarsi alla carriera. Al momento del congelamento degli oociti, il ricorso alla tecnologia è quindi dettato da motivi sociali e non da motivi medici.

La tecnologia medica del social freezing si articola su tre fasi successive. Durante la prima, le ovaie sono sottoposte a un trattamento di stimolazione ormonale (stimolazione ovarica), dopodiché sono punte per via vaginale mediante un ago e sono aspirati gli oociti. La seconda fase comprende la crioconservazione degli oociti, ossia il loro congelamento in azoto liquido a meno 196 gradi. Nella terza fase, gli oociti sono scongelati e fecondati in provetta (in vitro) con lo sperma del padre prescelto. La prima e la terza fase corrispondono alla classica fecondazione in vitro, praticata ormai da 40 anni. II congelamento degli oociti è invece praticato diffusamente (rendendo possibile la diffusione del social freezing) solo da poco più di dieci anni. A spianare la strada a questa evoluzione è stato un nuovo metodo di crioconservazione degli oociti: con la vitrificazione, gli oociti sono congelati rapidamente, in modo da evitare la formazione di pericolosi cristalli di ghiaccio al loro interno. I tassi di gravidanza nell'ambito della fecondazione (in vitro) di oociti crioconservati sono praticamente equivalenti a quelli raggiunti con oociti non congelati.

La tecnologia del social freezing permette di ridurre la pressione dell'orologio biologico sulle donne. Al tempo stesso solleva però interrogativi sui rischi medi$\mathrm{ci}$, suscita dubbi etici in relazione all'interesse del bambino e vanifica eventuali sforzi sociali volti a conciliare lavoro e famiglia.

Per illustrare le opportunità e i rischi della tecnologia in un'ottica globale, il presente studio ha combinato vari approcci metodologici. Per valutare le opportunità e i rischi medici nonché le conseguenze sociali, finanziarie e giuridiche del social freezing si è proceduto a un'analisi della letteratura. Per capire meglio 
l'offerta e la domanda di social freezing in Svizzera è stata realizzata un'indagine online interrogando tutti i centri di fertilità svizzeri. A titolo complementare sono inoltre state condotte interviste esplorative con medici della riproduzione e donne che hanno fatto congelare i loro oociti. Per sondare l'interesse nei confronti del social freezing in Svizzera e stimare il potenziale di questa tecnologia sono state intervistate online 408 donne in età compresa tra 25 e 39 anni senza figli. Gli atteggiamenti nei confronti del social freezing in seno alla società svizzera sono stati rilevati da un lato attraverso un'analisi dei media e dall'altro mediante interviste a 23 attori in rappresentanza di tutti i partiti politici nonché dei gruppi sociali rilevanti ai fini del social freezing. Infine, la legislazione svizzera è stata confrontata con quella applicabile in Austria, Francia, Italia, Germania, Belgio, Spagna e nella Repubblica Ceca. Qui di seguito sono presentati i principali risultati dello studio.

\section{Opportunità e rischi della tecnologia tuttora poco studiati}

La stimolazione ovarica e la fecondazione in vitro degli oociti sono praticate da ormai 40 anni. La crioconservazione degli oociti è invece un fenomeno più recente. I dati disponibili sui rischi della crioconservazione degli oociti per la salute del bambino sono quindi scarsi. I rischi associati alle altre due fasi della tecnologia sono invece stati studiati maggiormente. Nella discussione sul social freezing, tali rischi sono tuttavia spesso messi in secondo piano. I rischi associati alla stimolazione ovarica e alla fecondazione in vitro degli oociti sono preeclampsia, parto prematuro, basso peso alla nascita e un tasso di malformazioni leggermente superiore. Si ipotizza inoltre che questi metodi provochino più malattie tumorali in età pediatrica e compromettano lo sviluppo cognitivo del bambino. I metodi della medicina riproduttiva favoriscono infine la comparsa di alterazioni del sistema cardiocircolatorio correlate a malattie croniche e decessi in età adulta. L'entità e l'importanza di questi risultati della ricerca devono tuttavia ancora essere chiariti in altri studi epidemiologici di ampia portata.

Sia a livello internazionale sia a livello svizzero, le stime della probabilità di mettere al mondo un bambino con l'aiuto del social freezing si basano su ipotesi non sempre corrispondenti alla realtà. Per le donne di meno di 35 anni si stima una probabilità del 60-80 per cento congelando 15-20 oociti. Sia all'estero sia in Svizzera, tuttavia, per motivi di costi o a causa degli effetti collaterali del trattamento talvolta le donne fanno congelare nettamente meno oociti. Siccome 
finora sono poche le donne che hanno fatto ricorso ai loro oociti congelati, le prospettive di successo di questa tecnologia non possono ancora essere valutate in modo attendibile.

Domanda scarsa e motivata prevalentemente dall'assenza di un partner

Attualmente, la domanda di social freezing è scarsa. Si stima che le donne che fanno crioconservare i loro oociti in Svizzera siano da 300 a 400 all'anno. All'estero, tali donne hanno tendenzialmente più di 35 anni, sono single e hanno un buon livello di formazione. Anche in Svizzera il loro profilo dovrebbe essere simile.

Solo circa la metà delle donne in età compresa tra 25 e 39 anni senza figli intervistate conosce la tecnologia, la domanda è tuttavia in continua crescita. In Svizzera il 34 per cento delle donne senza figli intervistate è sostanzialmente interessata al social freezing. II sette per cento di esse manifesta un interesse concreto. II potenziale del social freezing dovrebbe quindi essere compreso tra 2000 e 10000 donne, che ogni anno farebbero crioconservare i loro oociti.

Le donne che farebbero fecondare in vitro i loro oociti congelati dovrebbero essere nettamente meno. Stando a studi internazionali, finora solo il 10 per cento delle donne ha fatto ricorso ai propri oociti crioconservati. Eppure circa un terzo delle donne con oociti congelati considera molto alta la probabilità di far fecondare in vitro i propri oociti.

Quale motivo per la crioconservazione degli oociti, le donne intervistate non menzionano la carriera, bensì l'assenza di un partner. Cercano un partner che corrisponda all'immagine dei «nuovi papà», che condividono con la madre la responsabilità per l'educazione dei figli e desiderano impegnarsi a fondo nella cura dei figli.

Ad attenuare l'interesse nei confronti del social freezing sono soprattutto le informazioni sui costi e i rischi per la donna e il bambino. Visti i rapidi mutamenti tecnologici e sociali in questo settore, le previsioni relative alla domanda sono tuttavia molto incerte. 
Basi per una decisione informata fondamentali dal punto di vista etico, ma non sempre garantite

La discussione etica sul social freezing ruota attorno all'interesse del bambino e all'importanza di una decisione informata a favore della tecnologia. Tutti i 23 attori sociali intervistati nell'ambito dello studio ritengono che l'offerta alle donne di una consulenza dettagliata costituisca una premessa molto importante per una decisione informata. Un'informazione esaustiva è particolarmente importante proprio per gli interventi facoltativi, senza un'indicazione medica. La maggior parte degli attori sociali intervistati teme però che gli offerenti influenzino la decisione delle donne a causa degli interessi commerciali in gioco. Una minoranza degli intervistati esclude invece tale influenza da parte degli offerenti. Dal presente studio emerge che singole donne non sono state informate in modo esaustivo sui rischi per la donna e il bambino.

L'età avanzata della madre al centro della discussione etica sull'interesse del bambino

La letteratura mette l'accento soprattutto sull'età della madre al momento della fecondazione in vitro degli oociti e i conseguenti rischi medici per il bambino. Anche i 23 attori svizzeri intervistati si schierano a favore di un limite di età per la fecondazione in vitro, principalmente a causa dei rischi medici. Altri motivi a favore di un limite di età, come le pari opportunità tra i sessi in relazione all'accesso alla medicina riproduttiva, le risorse delle madri più in là con gli anni nell'educazione del figlio nonché l'immagine della famiglia e le relazioni intergenerazionali mutate, svolgono un ruolo secondario. La maggioranza degli attori svizzeri intervistati è favorevole a un limite di età fisso.

Influsso del social freezing sugli sforzi sociali volti a conciliare il lavoro e la famiglia al centro della discussione sociale

La discussione sociale è imperniata sull'influsso del social freezing sulla conciliabilità tra lavoro e famiglia. Tra gli attori sociali intervistati e nei media svizzeri prevale lo scetticismo. Si teme che il social freezing subentri alle misure politiche, sociali o di dritto del lavoro volte a conciliare il lavoro e la famiglia e si ripercuota negativamente sulle pari opportunità tra i sessi. In base alle risposte 
delle donne e dei medici della riproduzione, tuttavia, il fattore determinante per il ricorso al social freezing è l'assenza di un partner adatto piuttosto che considerazioni sulla carriera. Stando così le cose s'impone una riflessione completa e sistematica sull'interazione tra il social freezing e le intenzioni di creare una famiglia sia delle donne sia degli uomini. Gli effetti di un ampio ricorso al social freezing per la salute pubblica, in particolare la frequenza di malattie non trasmissibili, non sono oggetto di discussione né a livello internazionale né a livello svizzero.

Esclusa un'assunzione dei costi sia del datore di lavoro sia dell'assicurazione malattie sociale

Sia a livello internazionale sia tra gli attori sociali intervistati in Svizzera vi è un ampio consenso sul fatto che né il datore di lavoro né l'assicurazione malattie sociale debbano essere chiamati a fornire un contributo alla crioconservazione degli oociti. Tale spesa deve essere assunta dalle donne stesse. L'argomento principale consiste nel dire che il ricorso al social freezing è dettato da motivi sociali e non da motivi medici. Gli attori intervistati si oppongono anche a una partecipazione ai costi della fecondazione in vitro nell'ambito del social freezing. Solo una minoranza non esclude una partecipazione ai costi per garantire pari opportunità alle donne con un reddito basso.

Disciplinamento giuridico del social freezing imperniato su un limite di età piuttosto che su un termine di conservazione

Un confronto con la legislazione sul social freezing in sette altri Paesi europei rivela due differenze essenziali: da un lato, la maggior parte dei Paesi applica un limite di età per la fecondazione in vitro degli oociti crioconservati. Dall'altro non vi è alcun Paese, ad eccezione del Belgio, in cui vige un termine per la conservazione degli oociti. A differenza della Svizzera, in Belgio la durata di conservazione può tuttavia essere prorogata in qualsiasi momento dai centri di fertilità. Se per il termine di conservazione non vi sono motivi medici, un limite di età per la fecondazione in vitro è invece giustificato sia da considerazioni mediche sia da considerazioni etiche, sociali e giuridiche. 
Nessun divieto del social freezing, ma ancora molti dubbi e incertezze

Nel complesso, in relazione al social freezing sussiste un ampio ventaglio di opinioni. Si tratta di una questione molto personale, strettamente legata a fattori individuali, come l'età, il sesso e la propria situazione in relazione al desiderio di procreare. Per la maggioranza dei 23 attori sociali intervistati, un divieto del social freezing è escluso, sussistono tuttavia molti dubbi e incertezze sull'applicazione di questa tecnica. Le opinioni in Svizzera non sembrano ancora definitive e occorrerà proseguire la discussione.

Sulla scorta dei risultati emersi dallo studio, il gruppo di progetto ha formulato, in collaborazione con il gruppo di accompagnamento, sei raccomandazioni.

\section{Raccomandazione 1: Applicazione moderata del social freezing}

Mediante il social freezing, tecnologie della medicina della procreazione sono applicate su donne sane - senza che ciò sia necessario dal punto di vista medico. Ciò comporta un'accresciuta responsabilità etica, in particolare a causa dei rischi per il bambino e degli effetti sulla salute pubblica. I dati disponibili sulle conseguenze della crioconservazione sono ancora scarsi. I risultati di studi recenti indicano inoltre che i metodi della medicina riproduttiva si ripercuotono negativamente sul sistema cardiovascolare del bambino. Prima di generalizzare l'applicazione del social freezing sono pertanto necessarie informazioni più affidabili sui rischi medici, sociali e psicologici per il bambino. Nel frattempo la tecnologia dovrebbe essere utilizzata e applicata con moderazione.

\section{Raccomandazione 2: Promozione della ricerca sui rischi del social freezing}

Per ottenere informazioni affidabili sui rischi medici e di altra natura della medicina della procreazione, bisognerebbe promuovere la ricerca sul social freezing, ad esempio nell'ambito di un programma nazionale di ricerca (PNR) del Fondo nazionale svizzero dedicato alla medicina della procreazione. Un programma del genere consentirebbe di studiare anche gli aspetti etici, sociali e giuridici del social freezing, oltre a quelli medici. Per studiare le conseguenze a lungo termine sono inoltre importanti cooperazioni internazionali, in modo da raggiungere un numero di soggetti sufficientemente alto e ottenere risultati rappresentativi. 
Raccomandazione 3: Garantire la registrazione di dati sul social freezing

Per poter valutare meglio la domanda di social freezing e le sue conseguenze sono necessari dati affidabili da parte delle cliniche e dei laboratori. Dovrebbero essere disponibili informazioni sul numero e l'età delle donne che ogni anno fanno crioconservare i loro oociti per motivi sociali. Bisognerebbe inoltre rilevare il numero e l'età delle donne che fanno fecondare in vitro gli oociti congelati. Dovrebbero infine essere raccolti e analizzati dati sui tassi di gravidanza e nati vivi nell'ambito del social freezing.

\section{Raccomandazione 4: Garantire una decisione informata}

Le donne interessate dovrebbero ricevere informazioni esaustive sul metodo medico, i rischi per la donna e il bambino, le prospettive di successo, i costi e le condizioni giuridiche quadro. Vi sono indicazioni secondo cui oggi i medici e le cliniche non sempre informano le donne interessate in misura sufficiente. L'ideale sarebbe offrire alle donne una consulenza indipendente ed esaustiva su tutti gli aspetti del social freezing. Converrebbe esaminare la possibilità di creare un'offerta informativa sul social freezing presso i centri cantonali di pianificazione familiare e salute sessuale.

Raccomandazione 5: Sostituzione del termine di conservazione con un limite di età

II termine massimo di dieci anni per la conservazione degli oociti congelati dovrebbe essere sostituito da un limite di età. Una gravidanza in età avanzata comporta rischi medici con conseguenze per la salute della madre e del bambino. La genitorialità in età avanzata è inoltre associata a rischi sociali. II diritto in materia di adozione prevede una differenza di età tra figli e genitori adottivi non superiore a 45 anni. Per garantire la congruenza tra il diritto in materia di adozione e quello in materia di procreazione, per le gravidanze dopo fecondazione in vitro bisognerebbe fissare un limite massimo di età di 45 anni. Con l'introduzione di un limite di età, gli oociti congelati sarebbero distrutti una volta raggiunto il limite di età e non più alla scadenza del termine di conservazione. 
Raccomandazione 6: Nessun finanziamento del social freezing attraverso le casse malati

I costi del congelamento degli oociti non dovrebbero andare a carico dell'assicurazione malattie. Per principio, le casse malati devono assumere i costi causati da malattie o dalla loro prevenzione. Una previdenza individuale per l'eventualità che sorga il desiderio di procreare una volta raggiunta l'infertilità dovuta all'età non può tuttavia rientrare nel concetto di prevenzione delle malattie. 


\section{Einleitung}

Social Freezing - auch Eigen-Eizellenspende genannt - ermöglicht Frauen dank der Kryokonservierung von Eizellen, ihren Kinderwunsch auch noch im höheren Alter zu verwirklichen. Damit nehmen eigentlich fruchtbare Frauen medizinisch unterstützte Fortpflanzung in Anspruch, um ihre Schwangerschaft zeitlich nach hinten zu versetzen, weil sie beispielsweise aufgrund ihrer Karriere oder eines fehlenden Partners vorher dazu nicht bereit sind.

Dem Alter der biologischen Mütter war bisher durch das Zeitfenster der natürlichen Fertilität Grenzen gesetzt, denn die Fruchtbarkeit wird stark vom Alter der Frau beeinflusst. Durch Social Freezing können heute Frauen ihre fruchtbare Phase verlängern, indem sie ihre Eizellen konservieren lassen. Hierzu werden innen, idealerweise vor einem Alter von 35 Jahren, reife Eizellen entnommen, eingefroren und eingelagert. Bei Bedarf können die Frauen die Eizellen dann Jahre später auftauen und per In-vitro-Fertilisation (IVF) befruchten lassen.

Entwickelt wurde diese Methode ursprünglich für Krebspatientinnen, um innen nach einer Chemo- oder Strahlentherapie eine Mutterschaft zu ermöglichen. Doch auch immer mehr gesunde Frauen wollen heute ohne medizinischen Grund dieses Fortpflanzungsverfahren in Anspruch nehmen - sozusagen als Versicherung für ihren späteren Kinderwunsch. Social Freezing ermöglicht damit Schwangerschaften bis ins fortgeschrittene Alter und erlaubt Frauen, auch nach der Menopause noch biologisch eigene Kinder zu bekommen.

Die Technologie verspricht, Frauen vom Zeitdruck der biologischen Uhr zu befreien, und ermöglicht innen dadurch, ihre Karriere voranzutreiben respektive einen passenden Partner zu finden. Gleichzeitig wirft Social Freezing Fragen zu medizinischen Risiken auf, löst ethische Bedenken bezüglich des Kindeswohls aus und untergräbt möglicherweise gesellschaftliche Bestrebungen zur Vereinbarung von Beruf und Familie. Ausgehend von diesem Spannungsfeld werden in der vorliegenden wissenschaftlichen Studie die Chancen und Risiken des Social Freezing aufgezeigt. 


\section{$1.1 \quad$ Forschungsfragen}

Die Studie wurde durch folgende Fragestellungen zur Technologie, zur Nachfrage sowie zu den ethischen, gesellschaftlichen, finanziellen und rechtlichen Rahmenbedingungen geprägt:

\section{Technologie}

- Welche Risiken bestehen für Frauen bei der ovariellen Stimulation und der Eizellentnahme?

- Welche Risiken bestehen für das Kind nach der In-vitro-Befruchtung?

- Wie werden diese Risiken durch die Technologie beeinflusst?

- Wie lange können die kryokonservierten Eizellen aufbewahrt werden und welche Folgen hat die Konservierung für die daraus entstandenen Kinder?

- Wie werden die gesundheitlichen Risiken für das Kind durch das Alter der Frau beeinflusst?

- Wie beeinflussen die Kryokonservierung und die Erfahrung der behandelnden Klinik die Erfolgsaussichten?

- Wie beeinflusst das Alter der Frau die Erfolgsaussichten?

- Welche Aspekte sollten im medizinischen Beratungsgespräch behandelt werden, um eine informierte Entscheidung für oder gegen Social Freezing sicherzustellen?

\section{Nachfrage}

- Wie viele Frauen nehmen in der Schweiz aktuell Social Freezing in Anspruch? Wie viele reisen dafür ins Ausland?

- Aus welchen Gründen nehmen Frauen Social Freezing in Anspruch? Welche Erwartungen haben Frauen an die Technologie?

- Wiegt Social Freezing Frauen in falscher Sicherheit?

- Wie, wann und unter welchen Voraussetzungen wollen Frauen später auf ihre kryokonservierten Eizellen zurückgreifen?

- Wie gross ist das Potenzial dieser Technologie in Zukunft? 


\section{Ethische Dimension des Social Freezing}

- Reicht es, wenn Frauen oder Paare eine gut informierte Entscheidung treffen, oder ist es Aufgabe der Gesellschaft oder des Staats, die Rahmenbedingungen wie den Verwendungszweck von Eizellen oder das Höchstalter der Frau festzulegen?

- Wie muss damit umgegangen werden, wenn Fortpflanzungsfreiheit und Kindeswohl in Konflikt zueinander stehen? Was ist höher zu werten und warum?

- Wie ist das Kindeswohl in Bezug auf die physische und psychische Entwicklung zu beurteilen, wenn ein grosser Altersunterschied zwischen Mutter und Kind besteht?

- Gibt es ein Recht auf ein Kind in jedem Lebensalter?

- Was soll mit den überzähligen eingefrorenen Eizellen passieren?

- Sind eine rein vorsorgliche Eizellentnahme und die anschliessende Vernichtung ethisch vertretbar?

- Sollte es möglich sein, entnommene Eizellen für eine allfällige Spende oder die Forschung zur Verfügung zu stellen?

- Sollen entnommene Eizellen für eine allfällige Eizellspende zur Verfügung gestellt werden?

- Stellt Social Freezing eine akzeptablere Alternative zur Eizellspende dar?

\section{Gesellschaftliche Dimension des Social Freezing}

- Wie wirkt sich Social Freezing auf die gesellschaftlichen Bemühungen zur Vereinbarkeit von Beruf und Familie sowie zur Förderung der Chancengleichheit zwischen den Geschlechtern aus?

- Inwiefern leistet Social Freezing einer Medikalisierung sozialer Probleme Vorschub?

- Wie würde sich eine Zunahme des Social Freezing auf die Familienbilder in der Gesellschaft auswirken?

Finanzielle Dimension des Social Freezing

- Wer hat welche ökonomischen Interessen am Social Freezing? 
- Können sich Unternehmen eine Kostenbeteiligung vorstellen und wie würde diese beurteilt?

- Sollen die Kosten von den Frauen oder von den Krankenkassen übernommen werden?

\section{Rechtliche Dimension des Social Freezing}

- Wie ist Social Freezing in der Schweiz zurzeit gesetzlich in Bezug auf Indikation, soziale Kriterien, Aufbewahrungsfrist sowie Alterslimite geregelt und wie unterscheidet sich diese Regelung von anderen Ländern?

- Inwiefern besteht ein Reformbedarf? Sollte statt der Aufbewahrungsfrist für die Eizellen eine Alterslimite für die Frauen festgelegt werden?

- Wenn ja, welche Alterslimite ist aus medizinischer, ethischer, gesellschaftlicher und rechtlicher Sicht angemessen?

- Soll die Befruchtung der Eizellen durch In-vitro-Befruchtung weiterhin nur heterosexuellen Paaren mit diagnostizierter medizinischer Unfruchtbarkeit vorbehalten bleiben oder sollten diese rechtlichen Voraussetzungen gelockert werden?

\subsection{Methodisches Vorgehen}

Zur Beantwortung der Forschungsfragen wurden sechs verschiedene Methoden angewendet, welche nachfolgend beschrieben werden.

\subsubsection{Literaturanalyse}

Für eine Auslegeordnung der medizinischen Chancen und Risiken sowie der ethischen, gesellschaftlichen, finanziellen und rechtlichen Folgen des Social Freezing wurde eine Literaturanalyse durchgeführt. Den Ausgangspunkt der Literaturanalyse bilden die Stellungnahmen von ärztlichen Fachgesellschaften wie der American Society for Reproductive Medicine (ASRM) sowie der European Society of Human Reproduction and Embryology (ESHRE) aus dem Jahr 2012. Diese Stellungnahmen bieten eine gute Übersicht über die bereits publi- 
zierten Artikel zu den medizinischen und ethischen Rahmenbedingungen des Social Freezing. Ergänzend zu diesen Stellungnahmen wurde nach wissenschaftlichen Artikeln gesucht, welche nach 2012 publiziert wurden. Die Suche erfolgte in den Datenbanken von Google Scholar, PubMed, JSTOR, SocialScience und PsychINFO. Als Schlagwörter wurden primär "Social Freezing», «Egg Freezing» und «Elective oocyte preservation» verwendet. Um Informationen zu den medizinischen Chancen und Risiken des Social Freezing zu finden, wurde zusätzlich nach Artikeln zu den perinatalen Outcomes, Health Outcomes und epigenetischen Risiken in Zusammenhang mit IVF und ICSI gesucht. Für die Beurteilung der medizinischen Risiken wurden hauptsächlich Übersichtsarbeiten wie Metaanalysen und systematische Reviews und keine Einzelstudien berücksichtigt. In den sozialwissenschaftlichen Datenbanken wurde zudem nach Informationen zu älteren Müttern gesucht. In den im Literaturverzeichnis aufgeführten Artikeln wurde entlang der Forschungsfragen nach Informationen gesucht, anschliessend wurden diese zusammengefasst (vgl. Anhang 9). Die Literaturanalyse wurde Ende 2017 abgeschlossen und im Laufe des Jahres 2018 punktuell ergänzt. Die Ergebnisse aus der Literaturanalyse sind in die Kapitel 2 bis 5 eingeflossen.

\subsubsection{Medienanalyse}

Um ein erstes Meinungsbild der Einstellung zum Social Freezing in der Schweizer Gesellschaft zu gewinnen, wurde eine Medienanalyse durchgeführt. Die Suche nach relevanten Artikeln geschah via Stichwortsuche ${ }^{1}$ in verschiedenen Datenbanken, wie WISO, LexisNexis und Factiva, mit Medien der deutschsowie französischsprachigen Schweiz. Diese wurde ergänzt mit einer auf die Schweiz begrenzten Internetrecherche zum Thema Social Freezing. Die Suche wurde auf Artikel beschränkt, die in der Periode von Ende 2011 bis Anfang 2017 erschienen sind. Nicht in die Analyse miteinbezogen sind Artikel, bei denen der Fokus auf der Reproduktionsmedizin im Generellen liegt und die Technik Social Freezing zwar erwähnt, aber nicht separat diskutiert wird. Reine

1 Stichwörter wie «Social Freezing», «egg freezing», «Kryokonservierung», «Eizellen einfrieren», «Congélation d'octocytes». 
Newsmeldungen und im Wortlaut identische Artikel wurden ebenfalls nicht in die Analyse integriert. Insgesamt umfasst die Medienanalyse 44 Artikel. 32 Artikel stammen aus Tages- und Wochenzeitungen, zwölf Artikel aus verschiedenen (Fach-)Zeitschriften und Onlinemedien (siehe Verzeichnis). Falls vorhanden, wurden auch Meinungen von Leserinnen und Lesern in die Analyse miteinbezogen. Die Medienartikel wurden entlang der Forschungsfragen ausgewertet. Die Ergebnissen der Medienanalyse werden in Kapitel 6 beschrieben.

\subsubsection{Onlinebefragung bei kinderlosen Frauen}

Zur Beurteilung des Potenzials von Social Freezing bei kinderlosen Frauen zwischen 25 und 39 Jahren in der Schweiz wurde Anfang 2018 von DemoSCOPE eine Onlinebefragung durchgeführt. Die Grundgesamtheit bestand aus allen Mitgliedern der DemoSCOPE-Community sowie dem Adresspool des DemoSCOPE-Partners CINT. Daraus wurde eine Zufallsauswahl von Frauen zwischen 25 und 39 Jahren kontaktiert.

Die Zielpersonen wurden per E-Mail eingeladen, an der Befragung teilzunehmen. Die E-Mail beinhaltete einen direkten Link zur Befragung. Das Befragungsthema wurde bewusst nicht erwähnt. Hingegen war es nötig, die Zielgruppe möglichst genau zu umschreiben, damit möglichst keine Person sich veranlasst sah, in die Befragung einzusteigen, die nicht zur Zielgruppe gehörte. Gesucht wurden deshalb «Frauen zwischen 25 und 39, die keine eigenen Kinder haben». Am Anfang der Befragung wurden diese Merkmale abgefragt und auf diese Weise sichergestellt, dass nur Personen, die gemäss ihren Angaben zur Zielgruppe gehören, an der Befragung teilnehmen können. Alle anderen wurden mit Dank verabschiedet. Beantworteten die Zielpersonen die Befragung nicht innert nützlicher Frist bis zum Schluss, wurden sie - teilweise mehrmals mit weiteren E-Mails erinnert. Knapp 90 Prozent der Frauen, die in die Umfrage einstiegen, beendeten sie auch. Insgesamt füllten 408 Frauen den Fragebogen aus. 


\section{Beschreibung der Stichprobe}

Wie aus Tabelle 1 hervorgeht, sind in der Stichprobe kinderlose Frauen im Alter von 35 bis 39 Jahren mit 28 Prozent übervertreten im Vergleich zur Bevölkerung. Kinderlose Frauen im Alter von 25 bis 29 Jahren sind hingegen untervertreten. Der Anteil der kinderlosen Frauen im Alter von 30 bis 34 Jahren entspricht mit 29 Prozent in etwa dem Anteil in der Bevölkerung.

Tabelle 1: $\quad$ Anzahl und Anteil kinderloser Frauen nach Altersgruppen (Vergleich Bevölkerung mit Stichprobe)

\begin{tabular}{|l|l|l|l|l|l|l|}
\hline & $\begin{array}{l}\text { Total } \\
\text { Frauen* }\end{array}$ & $\begin{array}{l}\text { Anteil } \\
\text { Frauen } \\
\text { ohne Kind }\end{array}$ & $\begin{array}{l}\text { Total } \\
\text { Frauen } \\
\text { ohne Kind }\end{array}$ & $\begin{array}{l}\text { Verteilung } \\
\text { Bevölkerung }\end{array}$ & $\begin{array}{l}\text { Total } \\
\text { Befragte }^{\star * *}\end{array}$ & $\begin{array}{l}\text { Verteilung } \\
\text { Befragung }\end{array}$ \\
\hline 25-29 Jahre & $280^{\prime} 238$ & $81 \%$ & $227^{\prime} 127$ & $51 \%$ & 178 & $44 \%$ \\
\hline 30-34 Jahre & $294^{\prime} 810$ & $46 \%$ & $136^{\prime} 648$ & $31 \%$ & 117 & $29 \%$ \\
\hline 35-39 Jahre & $292^{\prime} 197$ & $27 \%$ & $77^{\prime} 790$ & $18 \%$ & 113 & $28 \%$ \\
\hline Total & & & $441^{\prime} 565$ & $100 \%$ & 408 & \\
\hline
\end{tabular}

Quelle: ${ }^{*}$ BFS (2016), ${ }^{* *}$ BFS (2013), ${ }^{* \star *}$ Befragung Social Freezing 2018; $n=408$.

74 Prozent der befragten Personen wohnen in der Deutschschweiz, 26 Prozent in der Romandie. Ein Drittel verdient zwischen 4000 und 5999 Franken, ein Viertel unter 4000 Franken, 17 Prozent zwischen 6000 und 7999 Franken und jeweils 4 Prozent zwischen 8000 und 9999 Franken beziehungsweise über 10'000 Franken. 35 Prozent der Frauen sind Singles, 52 Prozent leben mit dem Partner oder der Partnerin zusammen und 13 Prozent sind verheiratet. Fast die Hälfte (48 \%) hat einen Bachelor oder Master als Abschluss, 23 Prozent haben das eidgenössische Fähigkeitszeugnis, 9 Prozent die Maturität, 17 Prozent einen Lehrabschluss und 3 Prozent die obligatorische Schulbildung.

Ausser dem Alter liegen keine Vergleichszahlen zur Grundgesamtheit (kinderlose Frauen im Alter zwischen 25 und 39 Jahren) vor. Zahlen zum Bildungsstand von Frauen in der Schweiz (mit/ohne Kinder) deuten jedoch darauf hin, dass in unserer Stichprobe höher ausgebildete Frauen stärker vertreten sind als in der Grundgesamtheit. Gemäss Zahlen des BFS verfügten 201740 Prozent der 25- bis 34-jährigen Frauen und 32 Prozent der 35- bis 44-jährigen Frauen 
über einen Hochschulabschluss. Eine obligatorische Schulbildung haben 8 respektive 12 Prozent der Frauen (BFS 2018).

Bei der Auswertung der Daten wurde auf eine Gewichtung verzichtet. Durch die Gewichtung einer Stichprobe reduziert sich deren Genauigkeit, denn sie erhöht Varianz, Standardabweichung und Standardfehler in den Berechnungen. Dies ist umso problematischer, je kleiner die Stichprobe ist. Da in dieser Analyse nicht nur auf der Ebene der gesamten Stichprobe $(n=408)$, sondern auch in Bezug auf Antworten oder Charakteristika Auswertungen gemacht wurden, schien die Gefahr eines Verlustes an Präzision zu hoch. ${ }^{2}$ Die Resultate der Onlinebefragung bei potenziell interessierten Frauen werden in Kapitel 4.2 dargelegt.

\subsubsection{Onlinebefragung bei Reproduktionsmedizinern}

Um Informationen zu Angebot und Nachfrage des Social Freezing, zum Beratungsgespräch und zum Reformbedarf aus Sicht der Reproduktionsmedizinerinnen und -mediziner zu gewinnen, wurde eine Onlinebefragung bei allen 30 Fertilitätskliniken in der Schweiz durchgeführt. Die Onlinebefragung wurde mit Qualtrics programmiert, was eine Beantwortung der Fragen mittels Computer, Tablet und Smartphone in verschiedenen Sprachen ermöglicht. Den Reproduktionsmedizinerinnen und -medizinern wurde der Link zur Umfrage in einem Mail zugestellt, welches auch ein Informationsschreiben von TA-SWISS umfasste. Die Onlinebefragung wurde zwischen dem 10. April und dem 8. Mai 2018 durchgeführt. Aufgrund der geringen Rücklaufquote wurde zweimal ein Erinnerungsmail an die Reproduktionsmedizinerinnen und -mediziner gesandt. Zudem wurde der Präsident der Schweizerischen Gesellschaft für Reproduktionsmedizin gebeten, die Reproduktionsmedizinerinnen und -mediziner zu einer Teilnahme an der Onlinebefragung zu motivieren. Im zweiten Erinnerungsmail wurde auf seinen Aufruf verwiesen. Trotz dieser Bemühungen liegen vollständige

2 Auch ist es nicht immer eindeutig, hinsichtlich welcher Komponente gewichtet werden soll. In dieser Analyse wäre wohl eine Gewichtung bezogen auf die Alterskategorie am sinnvollsten gewesen, weil das Alter einen Einfluss auf die Einstellung zum Social Freezing hat. Dies ist aber letztendlich eine Annahme. 
Angaben nur aus acht Fertilitätskliniken vor. Zu einzelnen Fragen liegen allerdings Antworten aus zwölf Fertilitätskliniken vor. Die Antworten wurden mit dem Statistikprogramm SPSS deskriptiv ausgewertet. Aufgrund des geringen Rücklaufs sind die in den Kapiteln 2.4 und 3.2.1 beschriebenen Ergebnisse allerdings mit Vorsicht zu interpretieren.

\subsubsection{Explorative Interviews}

Um vertiefte Informationen zu den Anbietern des Social Freezing zu gewinnen, wurden zusätzlich zur Onlinebefragung explorative qualitative Interviews mit je zwei Reproduktionsmedizinern aus der Deutschschweiz sowie je einem Reproduktionsmediziner aus der französisch- und italienischsprachigen Schweiz geführt (vgl. Kapitel 2.4). Die interviewten Reproduktionsmediziner gaben auch Auskunft zu den soziodemografischen Merkmalen sowie der Motivation der Frauen, die in der Schweiz ihre Eizellen einfrieren lassen. Über einen der interviewten Reproduktionsmediziner konnten zudem zwei Frauen rekrutiert und zu ihren Erfahrungen mit Social Freezing befragt werden (vgl. Kapitel 2.4 und 3.2). Die Rekrutierung von weiteren Frauen verlief erfolglos. Auf ein Informationsschreiben zur Studie, welche in einer Klinik an die Frauen abgegeben wurde, antwortete niemand. Auch die Rekrutierungsversuche über den Blog «SocialFreezing.de» und das Forum von Swissmom verliefen erfolglos.

\subsubsection{Q-Methode}

Zur Beurteilung der Akzeptanz des Social Freezing bei verschiedenen gesellschaftlichen Gruppierungen in der Schweiz wurde die Q-Methode angewendet. Die Q-Methode ist ein qualitativer Ansatz, welcher sich quantitativer Methodenanalyse bedient, um die Ergebnisse zu interpretieren. Durch das Clustering der Antworten interviewter Akteure können Meinungstypen zu einem Thema gebildet werden. Die Durchführung der Q-Methode lässt sich in vier Arbeitsschritte aufteilen: erstens die Auswahl der Statements zum Thema Social Freezing, zweitens das für die Q-Methode spezifische Sortierungsverfahren der Statements, drittens die Anwendung von statistischen Analyseverfahren zur Identifikation der Meinungstypen und viertens die Interpretation der Ergebnisse. 
Das Vorgehen der vorliegenden Studie entlang der vier Arbeitsschritte wird im Folgenden kurz beschrieben.

\section{a) Generieren von Statements}

Der erste Arbeitsschritt besteht aus dem Zusammenstellen einer möglichst breiten Auswahl von subjektiven Aussagen, die zum Thema Social Freezing in der Schweiz existieren (sog. Q-sample). Ziel ist es, dass das Q-sample die bestehenden Meinungen zu dem untersuchten Thema möglichst breit abbildet, auch wenn eine solche Auswahl nie vollständig sein kann. Nach Watts und Stenner (2005, S. 75) soll das Q-sample aus insgesamt 40 bis 80 Statements bestehen. Die Auswahl in der vorliegenden Studie besteht aus einer Sammlung von 51 Statements. Diese beinhalten eine jeweils unterschiedliche Aussage beziehungsweise Einstellung zum untersuchten Thema Social Freezing (siehe Liste mit den Statements in Tabelle 6 im Anhang).

Die Statements können auf unterschiedlichen empirischen Quellen basieren (Müller und Kals 2004). Entweder stammen sie direkt aus dem Alltagskontext der Akteure und finden sich in Interviews oder Medienartikeln wieder (naturalistic samples). Oder die Statements stammen aus Quellen ohne direkten Bezug zum Alltagskontext, wie aus Fachliteratur oder empirischen Studien (readymade Q-samples). In der vorliegenden Studie wurde eine Kombination von beiden Typen gewählt (hybrid type). Als Quelle dienten die Protokolle der explorativen Interviews mit Nutzerinnen des Social Freezing, die Medienanalyse sowie die Analyse der wissenschaftlichen Literatur. Bei der definitiven Auswahl der Statements wurde darauf geachtet, dass Aussagen zu den relevanten Dimensionen des Themas Social Freezing (ethische Dimension, gesellschaftliche Dimension, finanzielle und rechtliche Dimension) berücksichtigt werden (siehe Coogan und Herrington 2011, S. 25). 


\section{b) Gesprächsteilnehmende und Sortierungsverfahren}

In der vorliegenden Studie wurden persönliche Gespräche mit 23 Akteuren ${ }^{3}$ geführt. Diese wurden so ausgewählt, dass alle politischen Parteien, die auf nationaler Ebene vertreten sind, sowie möglichst alle für dieses Thema relevanten gesellschaftlichen Gruppierungen abgedeckt sind (siehe Liste der Gesprächsteilnehmerinnen und -teilnehmer in Anhang A3). ${ }^{4}$ Damit kann gewährleistet werden, dass ein möglichst breites Meinungsspektrum berücksichtigt wird.

Die im ersten Arbeitsschritt ausgewählten Statements wurden auf Karten gedruckt und den Gesprächspartnerinnen und -partnern vorgelegt. Die Personen hatten die Aufgabe, die Statements zu ordnen, indem sie diese auf einer LikertSkala entsprechend ihrer persönlichen Einstellung wie folgt priorisierten:

- In einem ersten Schritt mussten die Gesprächspartnerinnen und -partner die Aussagen auf drei Kategorien verteilen: erstens diejenigen Aussagen, mit denen sie einverstanden sind; zweitens diejenigen, mit denen sie nicht einverstanden sind; und drittens Aussagen, bei denen sie unsicher sind oder keine relevante Meinung haben.

- In einem zweiten Schritt mussten die Gesprächspartnerinnen und -partner eine weitere Differenzierung vornehmen. Jede Aussage musste in Abhängigkeit ihres Zustimmungsgrads auf der Likert-Skala platziert werden.

- In einem dritten Schritt wurde die sich dadurch ergebende Auslegeordnung der Statements (Q-sort) fotografisch festgehalten. Diese wurde anschliessend in die Software PQ-Method eingegeben, mit der die statistischen Berechnungen durchführt wurden.

In der vorliegenden Studie wurde ein erzwungenes Verteilungsverfahren (sog. forced Q-sort, siehe Müller und Kals 2004) angewendet. Dabei wird die Anzahl der Karten, die den jeweiligen Kategorien auf der Likert-Skala zugeteilt werden mussten, begrenzt (vgl. Abbildung 1). Die Gesprächspartnerinnen und -partner wurden gebeten, eine Verteilung gemäss der Abbildung vorzunehmen.

3 Die Referenzzahl für die Teilnehmenden einer Q-Studie liegt gemäss Müller und Kals (2004) zwischen 10 und 50 Teilnehmenden.

4 Die Gesprächsteilnehmenden gehören insgesamt 8 politischen Parteien und 17 gesellschaftlichen Gruppierungen an. 
Dieses Sortierungsverfahren zwingt die befragten Akteure zu einer zusammenhängenden Priorisierung der Statements. Die Teilnehmenden können die einzelnen Statements nur im Vergleich zu den anderen Statements einordnen. Dieser Entscheidungsprozess resultiert im subjektiven Meinungsbild der Person (Q-sort). Die Befragten wurden zusätzlich gebeten, ihren Entscheidungsprozess mündlich zu kommentieren. Die Kommentare wurden protokolliert und bei der anschliessenden Interpretation der Ergebnisse berücksichtigt.

Abbildung 1: $\quad$ Likert-Skala eines erzwungenen Verteilungsverfahrens von 51 Statements

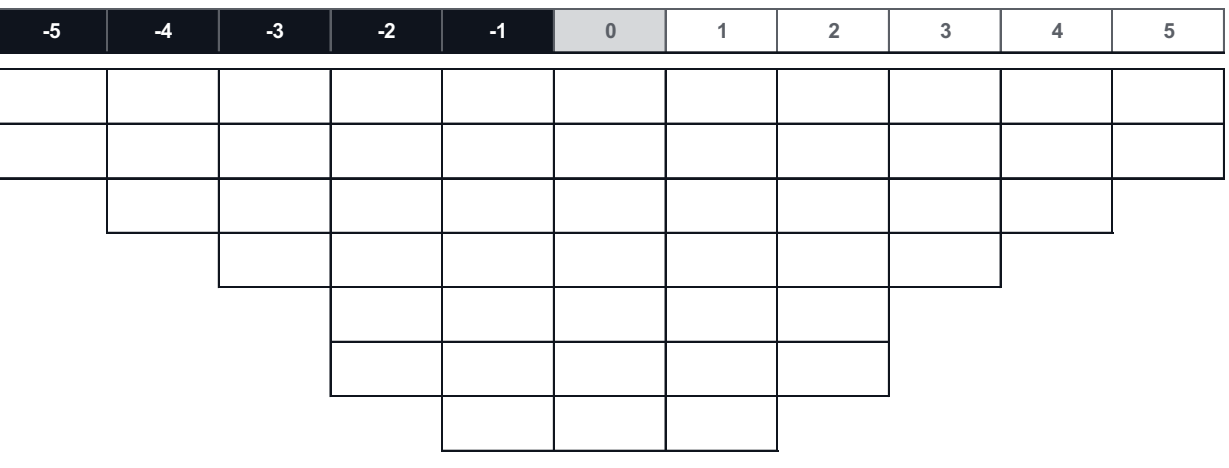

Quelle: eigene Darstellung. Legende: Auf den leeren Flächen wurden insgesamt 51 Statements auf einer Likert-Skala von -5 bis +5 platziert.

\section{c) Statistische Analyse}

Die statistische Analyse wurde mithilfe des Softwareprogramms PQ Method (Schmolck 2002) durchgeführt. Die Q-Methode kombiniert zwei statistische Verfahren, erstens eine Korrelationsberechnung sowie zweitens die Durchführung einer abgewandelten Form der Faktorenanalyse. ${ }^{5}$ Dabei werden nicht wie üblich Variablen, sondern Personen beziehungsweise ihre Meinungsbilder miteinander verglichen (Müller und Kals 2004). 
- Berechnung von Q-Korrelationen zwischen den Q-sorts: Für jedes Q-sortPaar wird ein Korrelationskoeffizient berechnet. Dadurch entsteht eine Korrelationsmatrix, welche die Beziehung eines jeden Q-sorts zu jedem anderen Q-sort zeigt (Watts und Stenner 2005, S. 80). Diese Korrelationsmatrix dient als Basis für die anschliessende Faktorenanalyse.

- Identifikation von Faktoren: Über die Anwendung von Faktorrotationstechniken werden ähnlich strukturierte Q-sorts zu einem gemeinsamen Faktor (Meinungstyp) zusammengefasst. ${ }^{6}$ Jedem Faktor können diejenigen Q-sorts zugewiesen werden, die dem identifizierten Meinungstyp am meisten entsprechen.

In der vorliegenden Studie wurden in der Interpretation der Ergebnisse nur diejenigen Faktoren berücksichtigt, die einen Eigenwert («eigenvalue») von deutlich grösser als 1.00 aufwiesen, ${ }^{7}$ sowie Faktoren, denen mindestens drei Qsorts mit signifikanter Faktorenladung ${ }^{8}$ zugewiesen werden konnten.

\section{d) Interpretation der Ergebnisse}

Folgende Punkte sind relevant, um die Meinungstypen zu interpretierten und qualitativ zu beschreiben, die den Faktoren zugrunde liegen.

- Beziehung der Meinungsbilder der Gesprächsteilnehmenden zu den Meinungstypen: Diese wird über die sogenannten Faktorenladungen (factor loadings) der einzelnen Q-sorts untersucht. Je höher die Faktorenladung, desto mehr korrespondiert das Q-sort mit dem identifizierten Meinungstyp.

- Beziehung einzelner Statements zu den Meinungstypen: Dies wird über die berechneten Faktorenwerte (factor scores) ausgedrückt. Ein hoher Faktorenwert bedeutet eine überdurchschnittlich positive Bewertung des State-

$6 \quad$ In der vorliegenden Studie wurde eine automatisierte Faktorenrotation (Varimax) durchgeführt.

7 Vgl. Watts und Stenner (2005), S. 81: «A standard requirement is to select only those factors with an eigenvalue in excess of 1.00. [...] A second standard requirement is that an interpretable $Q$ methodological factor must ordinarily have at least two $Q$ sorts that load significantly upon it alone.»

8 Die Formel für die Berechnung einer signifikanten Faktorenladung (mit $P<0.01$ ) ist 2.85 $(1 / \sqrt{ } \mathrm{n})$, wobei $\mathrm{n}$ der Anzahl Statements entspricht (Watts und Stenner 2011, S. 88). 
ments für den entsprechenden Meinungstyp, ein tiefer Wert eine überdurchschnittlich negative Bewertung (siehe Tabelle 6 im Anhang). Die Faktorenwerte der Statements, insbesondere die sehr hohen und sehr tiefen, verdeutlichen, welche Einstellungen typisch sind für jeden Meinungstyp. Schliesslich wird ein idealtypisches Q-sort für jeden Meinungstyp berechnet (Coogan und Herrington 2011, S. 26).

- Konsens- und Dissens-Statements: Charakteristische Aussagen («distinguishing statements») sind solche, welche die Teilnehmenden, die einen Meinungstyp repräsentieren, signifikant anders bewerten als Teilnehmende anderer Meinungstypen. Sie machen Unterschiede zwischen den Meinungstypen sichtbar. Genauso wichtig sind sogenannte «consensus statements», die auf Statements hinweisen, die bei allen Faktoren ähnlich bewertet wurden (vgl. dazu Coogan und Herrington 2011, S. 27).

Für die Interpretation der Ergebnisse wurden zusätzlich die Protokolle der Gespräche berücksichtigt. Die Ergebnisse und ihre Interpretation finden sich in Kapitel 7.

\subsubsection{Rechtsvergleichung}

Um die rechtlichen Rahmenbedingungen des Social Freezing in der Schweiz besser einordnen zu können, wurde eine Rechtsvergleichung mit sieben Ländern erstellt. Neben den vier Nachbarländern (Deutschland, Frankreich, Italien, Österreich) wurden drei weitere Länder miteinbezogen, welche eine wichtige Rolle im Fortpflanzungstourismus einnehmen (Belgien, Spanien, Tschechische Republik). Für die Rechtsvergleichung wurde nach wichtigen Literaturangaben, Erlassen und Materialien in den sieben Ländern gesucht. Aufgrund von sprachlichen Barrieren wurden für das Fallbeispiel Spanien, Italien und Tschechische Republik Expertinnen und Experten beigezogen, welche die relevanten Quellen sichteten und eine rechtliche Beurteilung des Social Freezing in ihrem Land vornahmen. Die Rechtsvergleichung wurde Ende 2017 abgeschlossen. Die Ergebnisse des Rechtsvergleichs werden im Kapitel 8 präsentiert. 


\section{Technologie}

In diesem Kapitel wird zuerst die Technologie im Zusammenhang mit Social Freezing erläutert (vgl. Kapitel 2.1). Danach werden die Risiken (vgl. Kapitel 2.2) und Erfolgsaussichten (vgl. Kapitel 2.3) beschrieben. Schliesslich wird auf die Anwendung der Technologie in der Schweiz eingegangen (vgl. Kapitel 2.4).

\subsection{Beschreibung der Technologie}

Unter Social Freezing wird das vorsorgliche Einfrieren eigener Eizellen bezeichnet, ohne dass dafür zum Zeitpunkt der Kryokonservierung eine medizinische Notwendigkeit vorliegt. Dies soll zur Überwindung des altersbedingten, ab 35 Jahren schnellen Abfalls der Fruchtbarkeit dienen. Die Eizellen können zu einem späteren Zeitpunkt aufgetaut und in vitro befruchtet werden. Dadurch können Frauen in einem Alter schwanger werden, in dem sie normalerweise bereits unfruchtbar wären. Anstelle von Social Freezing wird aus diesem Grund teilweise von einer Fertilitätsreserve aus sozialer Indikation gesprochen (Roth 2016). Unter sozialer Indikation werden nicht medizinische Gründe subsumiert, die gesunde Frauen dazu bewegen, ihre Eizellen einfrieren zu lassen, wie berufliche Pläne oder der fehlende Partner für die Familiengründung. Demgegenüber lassen Krebspatientinnen ihre Eizellen gestützt auf eine medizinische Indikation einfrieren.

Social Freezing stützt sich auf die Technologie der Kryokonservierung. Darunter wird das Einfrieren von Zellen oder Gewebe in flüssigem Stickstoff bei minus 196 Grad verstanden. Dadurch können diese über einen längeren Zeitraum ohne Beeinträchtigung ihrer Lebens- und Funktionsfähigkeit konserviert werden. Die Ursprünge der Kryokonservierung reichen bis in die Mitte des 20. Jahrhunderts zurück. Die ersten Versuche mit einer einfachen Kühltechnologie waren nicht erfolgreich, weil sie zu Zellschäden führte. In den 1940erJahren wurde allerdings entdeckt, dass die Beigabe von Glyzerol Spermien vor Schäden während der Kryokonservierung und des Auftauens schützt. In der Folge wurde 1953 von der ersten Geburt eines Babys berichtet, das mittels eines eingefrorenen Spermiums gezeugt wurde. In den 1970er-Jahren wurden 
weitere Substanzen identifiziert, welche die Zellschäden im Rahmen der Kryokonservierung minimieren. Zudem wurde die langsam programmierte Kryokonservierung (Slow Freezing) entwickelt. Dank dieser Verbesserungen war es möglich, Embryonen und Eizellen einzufrieren. Im Jahr 1984 wurde das erste Kind geboren, das aus einem eingefrorenen Embryo hervorging. Zwei Jahre später folgte die Geburt des ersten Kindes aus einer kryokonservierten Eizelle. Mit der damals praktizierten langsamen Einfriermethode waren die Erfolgsaussichten für das Überleben und die Befruchtung der Eizelle sowie für eine Schwangerschaft allerdings gering. Seit der Jahrtausendwende wurde jedoch mit der Vitrifikation ein neuartiges und Erfolg versprechenderes Verfahren entwickelt. Die Eizellen werden schockgefroren, wodurch die Bildung von zerstörerischen Eiskristallen in der Eizelle vermieden wird (ASRM 2012). ${ }^{9}$

Die Abbildung 2 bietet einen Überblick über die drei zeitlich abgegrenzten Phasen, die Bestandteil der Technik des Social Freezing sind. Grundsätzlich entspricht der Gesamtprozess eines Social Freezing einer klassischen In-vitroFertilisation, wobei beim Social Freezing der Ablauf mit der Gewinnung und anschliessenden Kryokonservierung der Eizellen unterbrochen wird.

Abbildung 2: $\quad$ Die drei Phasen des Social Freezing

\begin{tabular}{|c|} 
1. Phase: \\
$\begin{array}{c}\text { Ovarielle Stimulation und } \\
\text { Eizellentnahme }\end{array}$
\end{tabular}$\rightarrow \begin{gathered}\text { 2. Phase } \\
\begin{array}{c}\text { Kryokonservierung der } \\
\text { Eizellen }\end{array}\end{gathered} \rightarrow$\begin{tabular}{c} 
3. Phase \\
$\begin{array}{c}\text { Künstliche Befruchtung } \\
\text { der Eizellen }\end{array}$ \\
\hline
\end{tabular}

In einer ersten Phase erfolgt zuerst die Hormonbehandlung zur Stimulation der Eierstöcke. Danach werden diese über die Scheide mit einer Nadel punktiert

$9 \quad$ In dieser Studie liegt der Fokus auf der Kryokonservierung von Eizellen. Um die Fruchtbarkeit von Krebspatientinnen zu erhalten, werden heutzutage auch Eierstöcke entnommen. Die erste Geburt nach der Transplantation eines eingefrorenen Eierstocks erfolgte 2004. Die Geburtenrate liegt bei rund 24 Prozent pro Transplantat. Die Transplantation von Eierstöcken wird aber ebenfalls als Alternative zum Social Freezing diskutiert, da keine Befruchtung der Eizellen in vitro notwendig ist (von Wolff und Stute 2015). 
und die Eizellen abgesaugt. Die zweite Phase umfasst die Kryokonservierung der Eizellen. In der dritten Phase werden die Eizellen aufgetaut und mit den Spermien des Wunschvaters in der Glasschale (in vitro) befruchtet. Reproduktionsmedizinische Verfahren durchlaufen seit 40 Jahren die erste und die dritte Phase. Die Kryokonservierung der Eizellen wird hingegen erst seit gut zehn Jahren in grossem Stil angewendet. In den nachfolgenden Kapiteln werden die in diesen drei Phasen angewendeten Verfahren ausführlich beschrieben.

\subsubsection{Ovarielle Stimulation und Eizellentnahme}

In der ersten Phase des Social Freezing erfolgen die ovarielle Stimulation und die Eizellentnahme. Durch die ovarielle Stimulation soll das Wachstum der Eizellen angeregt werden. Dafür können unterschiedliche Stimulationsprotokolle angewendet werden. Um die Risiken in Zusammenhang mit der Stimulation zu reduzieren, wird in der Regel ein Antagonistenprotokoll verwendet. Im Rahmen dieses Protokolls spritzen sich die Frauen ab dem zweiten oder dritten Zyklustag selber ein Hormon (z.B. follikelstimulierendes Hormon) unter die Haut, welches das Wachstum der Follikel begünstigt. In der Regel ab dem fünften Stimulationstag wird zusätzlich zum ersten Hormon ein weiteres Hormon (GnRHAntagonist) gespritzt, um einen vorzeitigen Eisprung zu verhindern. Während der Stimulationsphase erfolgen durchschnittlich zwei Kontrollen mittels Ultraschall und Blutentnahme beim Arzt. Wenn der Leitfollikel eine Grösse von 18 bis 20 Millimetern erreicht hat, spritzt die Frau ein drittes Hormon (z.B. humanes Choriongonadotropin), um den Eisprung auszulösen. Rund 36 Stunden später werden die Eizellen während eines fünf- bis zehnminütigen Eingriffs unter Kurznarkose entnommen und eingefroren (Nawroth 2015).

\subsubsection{Kryokonservierung der Eizellen}

Die zweite Etappe des Social Freezing umfasst die Kryokonservierung der Eizellen. Die ersten Eizellen wurden in den 1980er-Jahren mit der langsam programmierten Kryokonservierung eingefroren (ASRM 2012). Bei dieser Methode wird zuerst das Wasser in der Eizelle mittels einer kryoprotektiven Lösung entzogen. Dafür bleibt die Eizelle mehrere Minuten in dieser Lösung. Durch den 
Entzug des Wassers in der Eizelle wird verhindert, dass sich während des Einfrierens der Eizelle Eiskristalle bilden und diese die Eizelle beschädigen (Nawroth 2015). Trotzdem waren mit dieser Methode die Überlebensraten der Eizellen nach dem Auftauen sowie die Schwangerschaftsraten unbefriedigend (Schattman 2017).

Durch die Methode der Vitrifikation konnte die Eizellkonservierung stark verbessert werden. Die Vitrifikation wurde nach der Jahrtausendwende entwickelt und ist heute die Methode der Wahl für das Einfrieren von Eizellen, trotz stetiger Verbesserungen in der langsam programmierten Kryokonservierung (ASRM 2013, Nawroth 2015). Bei der Vitrifikation muss das Wasser den Eizellen nicht vollständig entzogen werden, weil der Vitrifikationsvorgang so schnell abläuft, dass sich keine Eiskristalle in der Eizelle bilden können (Nawroth 2015). Im Vergleich zur langsam programmierten Kryokonservierung erfolgt das Einfrieren der Eizellen bei der Vitrifikation tausendmal schneller. Dafür muss bei der Vitrifikation eine deutlich höhere Konzentration der kryoprotektiven Lösung verwendet werden (Schattman 2017). Die Eizellen werden zuerst in eine Vitrifikationslösung getaucht, die zu gleichen Teilen aus Dimethylsulfoxid und Ethylenglykol besteht und mit Zucker sowie Protein versetzt ist. Danach werden die Eizellen auf ein Trägersystem aufgebracht. Schliesslich werden die Eizellen direkt (offenes System) oder nach Verschluss des Trägersystems (geschlossenes System) in flüssigen Stickstoff eingetaucht und auf minus 196 Grad abgekühlt. Die Flüssigkeit sieht nach der Vitrifikation klar und durchscheinend aus. Die Namensgebung dieser Methode ist auf diesen glasähnlichen (lateinisch vitrum für Glas) Zustand der Flüssigkeit zurückzuführen (Nawroth 2015).

\subsubsection{Befruchtung der Eizellen in vitro}

In der dritten Phase des Social Freezing werden die Eizellen in vitro befruchtet. Dafür werden sie zuerst aus dem flüssigen Stickstoff genommen und in einer Zuckerlösung inkubiert. Die Verwendung einer Zuckerlösung während des Erwärmens der Eizellen ermöglicht einen kontrollierten Entzug des Konservierungsmittels in der Eizelle, das durch Wasser ersetzt wird (Nawroth 2015). 
Nach dem Auftauen werden die Eizellen in der Glasschale mit den Spermien des Partners oder eines fremden Spenders befruchtet. Da diese im Prinzip meist fruchtbar sind, könnte die Befruchtung theoretisch durch eine In-vitroFertilisation (IVF) erfolgen. Bei diesem Verfahren werden das Sperma und die Eizelle in der Glasschale zusammengebracht und es erfolgt eine spontane Befruchtung. Bei einer Vitrifikation werden jedoch die Cumuluszellen, welche die Eizelle umgeben, vor dem Einfrieren entfernt. Dadurch kann die Reife der Eizellen beurteilt werden (Nawroth 2015). Da sich unreife Eizellen nach dem Auftauen nicht weiterentwickeln, sollten möglichst nur reife, befruchtungsfähige Eizellen eingefroren werden (Doyle et al. 2016). Die Entfernung der Cumuluszellen kann dazu führen, dass sich die Eizellen mit einer klassischen IVF schlechter befruchten lassen (ASRM 2013). Aus diesem Grund erfolgt die Befruchtung der Eizellen nach einer Vitrifikation mittels intrazytoplasmatischer Spermieninjektion (ICSI). Bei dieser Methode wird ein einzelnes Spermium mittels Pipette in die Eizelle eingebracht. Die intrazytoplasmatische Spermieninjektion wurde ursprünglich in erster Linie zur Behandlung der männlichen Unfruchtbarkeit entwickelt.

\section{$2.2 \quad$ Risiken}

Die Abbildung 3 bietet einen Überblick über die in Zusammenhang mit den drei Phasen des Social Freezing diskutierten kurz- und langfristigen Risiken für Mutter und Kind. Die Pfeile zeigen auf, ob die Technologie zu einem geringen Risiko (grün), einem nicht abschliessend geklärten erhöhten Risiko (orange), einem nachweisbar erhöhten Risiko (rot) oder einem kaum untersuchten Risiko (grau) für die Frau, die Eizellen einfrieren lässt, sowie ihre künftigen Kinder führt. Im Grundsatz entsprechen die nachfolgend aufgeführten Risiken denjenigen einer In-vitro-Fertilisation, wie sie seit rund 40 Jahren bei unerfülltem Kinderwunsch angewendet wird, und sind nicht spezifische Risiken des Social Freezing. 
Abbildung 3: $\quad$ Übersicht über die medizinischen Risiken für Frau und Kind in Zusammenhang mit Social Freezing

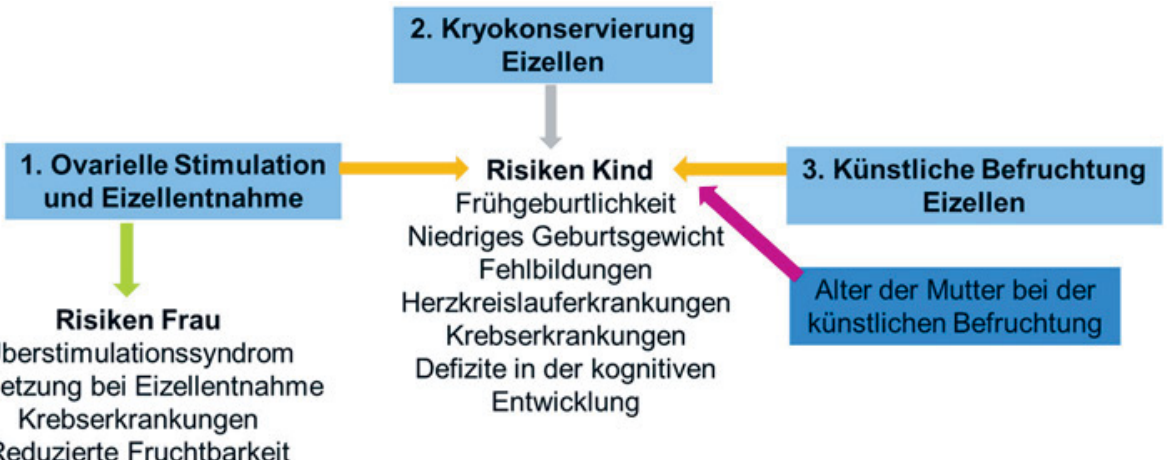

Legende: grüne Pfeile = geringes Risiko; orange Pfeile = nicht abschliessend geklärtes erhöhtes Risiko; rote Pfeile = erhöhtes Risiko; graue Pfeile = Risiko kaum untersucht.

Die erste Phase des Social Freezing - die ovarielle Stimulation - ist nachweislich mit geringen kurzfristigen Risiken für die Frau wie Überstimulationssyndrom und Verletzungen bei der Eizellentnahme verbunden. Für längerfristige Risiken für die Frau wie Krebs oder eine Einschränkung der Fruchtbarkeit gibt es keine Hinweise (grüne Pfeile).

Hingegen wurde die ovarielle Stimulation in mehreren Studien mit erhöhten Risiken für das Kind in Verbindung gebracht. Dies gilt auch für die dritte Phase des Social Freezing, welche die Befruchtung der Eizellen in vitro umfasst. Eine rasch wachsende Zahl von neueren Untersuchungen zeigt, dass neben anderen möglichen Einflüssen, wie zum Beispiel elterlicher Subfertilität und mütterlichem Alter, die reproduktionsmedizinische Technologie selbst signifikante Risiken mit sich bringt. Es mehren sich zurzeit die Hinweise, dass durch die ovarielle Stimulation oder die In-vitro-Befruchtung ausgelöste epigenetische Veränderungen diese Risiken erklären. Von solchen epigenetischen Veränderungen wären auch die Kinder von Frauen betroffen, die Eizellen aus sozialen Gründen einfrieren lassen (orange Pfeile). 
Während die ovarielle Stimulation und die In-vitro-Befruchtung seit mittlerweile 40 Jahren praktiziert werden, ist die breite Anwendung der Kryokonservierung von Eizellen eine neuere Erscheinung. Entsprechend wenige Daten liegen zurzeit zu den Risiken dieser Phase des Social Freezing vor. Die bisherigen spärlichen Daten zeigen jedoch, dass die Risiken für das Kind gering erscheinen (graue Pfeile).

Unabhängig von der Technologie bildet das Alter der Frau bei der Befruchtung der Eizellen in vitro einen Risikofaktor. Beim Social Freezing lässt eine Frau ihre Eizellen in der Regel in bereits fortgeschrittenem Alter in vitro befruchten. Die Risiken in Zusammenhang mit Schwangerschaft und Geburt steigen jedoch mit dem Alter der Mutter. Kinder von älteren Müttern haben ein höheres Risiko, zu früh geboren zu werden respektive ein zu tiefes Geburtsgewicht aufzuweisen. Studien belegen, dass diese beiden perinatalen Risiken wiederum HerzKreislauf-Erkrankungen sowie Defizite in der kognitiven Entwicklung begünstigen (roter Pfeil).

Nachfolgend werden die Studienergebnisse zu diesen Befunden im Detail beschrieben. Zuerst werden die Risiken für die Frau erläutert (vgl. Kapitel 2.2.1). Danach werden die gesundheitlichen Risiken für das Kind in Zusammenhang mit ovarieller Stimulation und In-vitro-Befruchtung beschrieben (vgl. Kapitel 2.2.2). Schliesslich wird spezifisch auf die Risiken in Zusammenhang mit Social Freezing eingegangen (vgl. Kapitel 2.2.3).

\subsubsection{Risiken der Reproduktionsmedizin für die Frau}

In Zusammenhang mit der ovariellen Stimulation und der Eizellentnahme werden vier Risiken für die Frau diskutiert.

Ovarielles Überstimulationssyndrom: Die Hormonstimulation kann zu einer übermässigen Grössenzunahme der Eierstöcke und zu einer Flüssigkeitsverschiebung in den Bauchraum und zwischen die Lungenblätter führen. Diese Flüssigkeitsverschiebung begünstigt die Eindickung des Blutes, die mit einem erhöhten Risiko für Thrombosen und Embolien verbunden ist (Roth 2016). Das Überstimulationssyndrom kann zudem zu Atemnot sowie Leberfunktionsstörungen führen. Eine solch schwere Ausprägung ist jedoch selten und liegt bei unter 
1 Prozent Wahrscheinlichkeit des Auftretens (Nawroth 2016). In Rahmen des Social Freezing dürfte das Risiko aus zwei Gründen noch tiefer liegen. Erstens wird mit den entnommenen Eizellen nicht sofort eine Schwangerschaft angestrebt. Darum besteht beim Social Freezing auch kein Risiko, am besonders problematischen, spät auftretenden Überstimulationssyndrom zu erkranken, das in Zusammenhang mit einer Frühschwangerschaft auftritt (ASRM 2012, Nawroth 2016, Wunder 2012). Zweitens können die Risiken mit dem oben beschriebenen Stimulationsprotokoll weiter minimiert werden (Nawroth 2016, Wunder 2012).

Komplikationen bei der Eizellentnahme: Die Entnahme der Eizellen kann zu verschiedenen Komplikationen wie vaginalen Blutungen, Verletzungen der Nachbarorgane, Infektionen und Problemen in Zusammenhang mit der Anästhesie führen. Dieses Risiko liegt ebenfalls bei unter 1 Prozent der Wahrscheinlichkeit, dass es auftritt (Nawroth 2016, Roth 2016, Wunder 2012).

Erhöhtes Krebsrisiko: Die im Rahmen der ovariellen Stimulation eingesetzten Medikamente stehen unter Verdacht, das Krebsrisiko für die Frau zu erhöhen. Gestützt auf eine systematische Literaturübersicht kommt die amerikanische Gesellschaft für Reproduktionsmedizin (ASRM 2012) zum Schluss, dass kein erhöhtes Risiko besteht, nach einer Fertilitätsbehandlung an einem bösartigen Krebs in der Brust, den Eierstöcken oder der Gebärmutterschleimhaut zu erkranken. In mehreren Studien wurde allerdings ein leicht erhöhtes Risiko für Borderline-Tumoren in den Eierstöcken nach einer Fertilitätsbehandlung nachgewiesen. Diese «grenzwertigen» Tumoren sind zwar bösartig, aber nicht sehr invasiv. Die Heilungsaussichten sind gut. Aus diesem Grund spricht sich die ASRM dagegen aus, die im Rahmen einer Fertilitätsbehandlung eingesetzten Medikamente nicht mehr zu nutzen.

Beeinträchtigung der Fertilität: Zudem wurde die Befürchtung geäussert, ovarielle Stimulation und die Eizellentnahme könnten die künftige Fertilität der behandelten Frauen beeinträchtigen. Dafür gibt es allerdings bislang keine Hinweise (Académie nationale de médecine 2017). 


\subsubsection{Risiken der Reproduktionsmedizin für das Kind}

Die ovarielle Stimulation und die In-vitro-Befruchtung werden seit 40 Jahren angewendet. Entsprechend liegen relativ viele Daten zu deren Einfluss auf verschiedene Risiken für das Kind vor. Diese werden nachfolgend erläutert. In einem weiteren Kapitel gehen wir auf zusätzliche Risiken in Zusammenhang mit Social Freezing wie die Kryokonservierung der Eizellen sowie das fortgeschrittene Alter der Frau bei der Befruchtung ihrer Eizellen in vitro ein (vgl. Kapitel 2.2.3).

\section{Perinatale Risiken}

Das grösste Risiko in Zusammenhang mit Schwangerschaft und Geburt nach einer Befruchtung der Eizellen in vitro betrifft die erhöhte Rate an Mehrlingsschwangerschaften. Mehrlingsschwangerschaften sind im Vergleich zu Einlingsschwangerschaften mit einer erhöhten perinatalen Morbidität und Mortalität verbunden (Wunder 2014). Die Befruchtung der Eizellen in vitro führt im Vergleich zur natürlichen Zeugung zu signifikant mehr Zwillingen und Drillingen, weil in der Regel mehr als ein Embryo in die Gebärmutter transferiert wird. Im Prinzip wäre dieses Risiko einfach zu kontrollieren, indem lediglich ein Embryo pro Behandlungszyklus verwendet wird. Weltweit besteht denn auch eine Tendenz, nur noch einen Embryo pro Behandlungszyklus zu transferieren (Wunder 2014).

Aber auch Einlingsschwangerschaften nach einer In-vitro-Befruchtung sind im Vergleich zu einer Spontankonzeption mit erhöhten Risiken verbunden (Chen und Heilbronn 2017). Bereits im Jahr 2004 zeigten drei Metaanalysen ein erhöhtes Risiko eines niedrigen oder sehr niedrigen Geburtsgewichts bei in vitro gezeugten Einlingen auf (Jackson et al. 2004; Helmerhorst et al. 2004; McGovern 2004, zitiert in Wunder 2014). In einer weiteren Metaanalyse konnte ein erhöhtes Risiko einer Frühgeburt beobachtet werden (Grady et al. 2012, zitiert in Wunder 2014). In einer gross angelegten Studie in Australien wurde zudem bei in vitro gezeugten Einlingen ein erhöhtes Risiko von niedrigem Geburtsgewicht, Frühgeburtlichkeit und neonataler Mortalität nachgewiesen (Marino et al. 2014). Zu ähnlichen Befunden gelangte eine weltweite Metaanalyse, die 52 Kohortenstudien einschloss und Daten von 181'741 Kindern nach Invitro-Befruchtung sowie von 4'636'508 Kindern nach Spontankonzeption um- 
fasste (Qin et al. 2017b). Auch bei natürlich gezeugten Kindern erhöhen solche Komplikationen rund um die Geburt das Risiko von Herz-Kreislauf-Erkrankungen, wie Bluthochdruck, Herzinsuffizienz, Herzinfarkt und Schlaganfall, und Todesfällen aufgrund von Herz-Kreislauf-Erkrankungen später im Leben signifikant (Scherrer et al. 2015).

\section{Angeborene Fehlbildungen}

In einer umfangreichen Literaturübersicht wurden verschiedene zwischen 2001 und 2013 publizierte Studien zu angeborenen Fehlbildungen nach In-vitroBefruchtung identifiziert (Fauser et al. 2014). Gemäss diesem Literatur-Review kamen drei von vier Metaanalysen zum Schluss, dass bei mittels IVF und ICSI gezeugten Kindern häufiger Fehlbildungen auftreten als bei natürlich gezeugten Kindern (Rimm et al. 2004; Hansen et al. 2005; Wen et al. 2012, zitiert in Fauser et al. 2014). Allerdings ist das Fehlbildungsrisiko gemäss einer Metaanalyse auch bei infertilen Paaren erhöht, die ohne medizinische Unterstützung Kinder bekommen (Rimm et al. 2011, zitiert in Fauser et al. 2014).

Auch Einzelstudien gelangen zu ähnlichen Erkenntnissen. In Australien sind zwei grosse Studien, basierend auf nationalen Registerdaten, erschienen. In der ersten Studie wiesen die 6163 mittels In-vitro-Befruchtung gezeugten Kinder ein deutlich erhöhtes Risiko von Fehlbildungen auf im Vergleich zu den 308'974 natürlich gezeugten Kindern. Die Fehlbildungsrate belief sich nach Invitro-Befruchtungen auf 8,3 Prozent, während sie bei einer natürlichen Befruchtung nur bei 5,8 Prozent lag (Davies et al. 2012, zitiert in Fauser et al. 2014 und Nawroth 2015). In einer zweiten Studie wurde die Fehlbildungsrate nach 2211 IVF, 1399 ICSI und 301'060 Spontankonzeptionen verglichen. Bei Spontankonzeption stieg das Risiko von Fehlbildungen mit dem Alter der Mutter. Nach einer Befruchtung mittels IVF oder ICSI war das relative Risiko von Fehlbildungen grösser als nach einer natürlichen Zeugung, wenn die Frauen unter 29 Jahre alt waren. Bei Frauen in der Altersgruppe 35 bis 39 zeigten sich keine Unterschiede im Fehlbildungsrisiko zwischen den beiden Zeugungsarten. Bei den Kindern von über 40-jährigen Frauen traten weniger Fehlbildungen nach einer In-vitroBefruchtung auf als nach einer natürlichen Zeugung (Davis et al. 2017).

Kleinere Einzelstudien gelangen zu widersprüchlichen Ergebnissen. Von den 21 identifizierten Kohortenstudien zeigten elf ein signifikant erhöhtes Fehlbildungs- 
risiko nach In-vitro-Befruchtung auf. In zehn Studien wurde hingegen nur ein leicht erhöhtes oder gar kein Risiko beobachtet. Auch die retrospektiven Studien gelangten zu widersprüchlichen Befunden (Fauser et al. 2014). Die Autoren des Literatur-Reviews gelangen zum Schluss, dass die Interpretation der Daten schwierig sei, weil sich die Studien bezüglich Methodologie sowie Definition, Klassifizierung und Erfassung der Fehlbildungen voneinander unterscheiden.

\section{Körperliche Entwicklung}

Die Studien zum Wachstum von in vitro gezeugten Kindern gelangen zu beruhigenden Ergebnissen. In der Mehrheit der Studien wurden keine Unterschiede im Wachstum zwischen natürlich und in vitro gezeugten Kindern beobachtet (Chen und Heilbronn 2017). Sowohl Grösse als auch Gewicht sind ähnlich (Woldringh et al. 2011a; Kai et al. 2006; Basatemur et al. 2006, zitiert in Catford et al. 2017). Ebenso sind keine nennenswerten Unterschiede bezüglich Knochenmineralisierung und Pubertät feststellbar (Fauser et al. 2014). Allerdings gelangen einzelne Studien zum Schluss, dass mittels In-vitro-Befruchtung gezeugte Kinder ein beeinträchtigtes respektive ein verstärktes Wachstum zeigen. Es kann jedoch nicht ausgeschlossen werden, dass diese Resultate dadurch beeinflusst wurden, dass einige der Studien Kinder einschlossen, die zu früh geboren wurden, ein tiefes Geburtsgewicht aufwiesen oder aus Mehrlingsschwangerschaften stammten (Chen und Heilbronn 2017).

\section{Krebserkrankungen}

Es gibt Hinweise auf einen Zusammenhang zwischen In-vitro-Befruchtung und der Entwicklung von Krebs bei Kindern. Während die ersten Studien zu beruhigenden Ergebnissen gelangten, wurden im letzten Jahrzehnt verschiedene Studien insbesondere in skandinavischen Ländern publiziert, die auf ein erhöhtes Risiko für bestimmte Krebsarten nach einer In-vitro-Befruchtung hindeuten (Chen und Heilbronn 2017). Eine grosse Metaanalyse zeigt zudem ein erhöhtes Risiko von Krebs und insbesondere von hämatologischen Krebserkrankungen (z.B. Leukämie) und Gehirntumoren nach einer In-vitro-Befruchtung (Hargreave et al. 2013, zitiert in Catford et al. 2017). In den meisten bisher publizierten Studien zum Krebsrisiko infolge Befruchtung der Eizellen in vitro wurden jedoch 
weitere mögliche Einflussfaktoren auf die Inzidenz der Krebserkrankungen nicht untersucht, wie der perinatale Gesundheitszustand, der sozioökonomische Status sowie mütterliches Rauchen (Chen und Heilbronn 2017).

\section{Herz-Kreislauf-Erkrankungen}

Ebenso scheint die In-vitro-Befruchtung mit einem erhöhten Risiko für HerzKreislauf-Erkrankungen verbunden zu sein (Wunder 2014). So zeigte die Untersuchung einer Forschergruppe des Inselspitals Bern an 65 in vitro gezeugten Kindern, dass diese im Vergleich zu 57 natürlich gezeugten Kindern eine steifere Armarterie sowie eine verdickte Innenschicht der Halsschlagader hatten und der Blutdruck in der Lungenschlagader erhöht war. Die Kinder beider Untersuchungsgruppen wiesen ein durchschnittliches Alter von elf Jahren auf. Gefässveränderungen im Kindesalter sind ein erstes Anzeichen für eine früh auftretende Kreislauferkrankung im Erwachsenenalter (Scherrer et al. 2012).

Die Gefässveränderungen können zudem zu einem erhöhten Blutdruck bereits in der Kindheit führen. Die Forschergruppe aus dem Inselspital hat die in der ersten Studie untersuchten Kinder im Alter von durchschnittlich 17 Jahren erneut untersucht und ihren Blutdruck überprüft. Die 54 in vitro gezeugten Kinder wiesen im Durchschnitt höhere Blutdruckwerte auf als die Kontrollgruppe von 43 natürlich gezeugten Kindern. Bei acht der 54 in vitro gezeugten Kinder wurde sogar Bluthochdruck festgestellt. In der Kontrollgruppe wies nur eines von 43 Kindern Bluthochdruck auf (Meister et al. 2018). Auch in anderen Studien wurde ein im Vergleich zu natürlich gezeugten Kindern erhöhter Blutdruck bei in vitro gezeugten Kindern aufgezeigt (Ceelen et al. 2008; Sakka et al. 2010; La Bastide-Van Gemert et al. 2013, zitiert in Chen und Heilbronn 2017). Im Rahmen einer Metaanalyse mit Daten von 2212 in vitro und 4096 natürlich gezeugten Kindern wurde eine kleine, aber signifikante Blutdruckerhöhung festgestellt (Guo et al. 2017).

In einer niederländischen Studie mit je 225 in vitro und natürlich gezeugten Kindern wurde zudem eine erhöhte Konzentration von Zucker in nüchternem Zustand bei den in vitro gezeugten Kindern gefunden. Andere Anzeichen für eine Insulinresistenz respektive die Entwicklung von Diabetes wurden allerdings nicht identifiziert (Ceelen et al. 2008, zitiert in Fauser et al. 2014). In einer ande- 
ren Studie wurde hingegen eine Insulinresistenz bei jungen in vitro gezeugten Erwachsenen beobachtet (Chen et al. 2014, zitiert in Scherrer et al. 2015).

Die bei in vitro gezeugten Kindern beobachteten Gefässveränderungen, der erhöhte Blutdruck sowie erste Anzeichen von Diabetes sind wichtige Risikofaktoren für Herzinfarkte, Schlaganfälle und gefässbedingte Demenz. Zum jetzigen Zeitpunkt ist die Evidenz allerdings noch nicht gefestigt, weil die bisherigen Studien nur kleine Studienpopulationen untersuchten und aufgrund des unterschiedlichen Studiendesigns zu nur teilweise vergleichbaren Ergebnissen gelangten (Chen und Heilbronn 2017). Ihre Bedeutung wird aber durch aussagekräftige Daten zu möglichen schädigenden Mechanismen in Tierversuchsmodellen bekräftigt. Weitere grössere Kohortenstudien, basierend auf umfangreichen Register- oder Kontrollstudien, werden als wichtig erachtet. Gestützt auf die bisherigen Erkenntnisse wird allerdings empfohlen, dass die Zeugungsmethode als Risikofaktor für Herz-Kreislauf-Erkrankungen systematisch erfasst wird (Weinrauch et al. 2018).

\section{Kognitive Entwicklung}

Bisher liegen aus Kohortenstudien aus Europa und den USA sowie einem systematischen Review von 57 Einzelstudien keine Hinweise auf ein erhöhtes Risiko schwerer kognitiver Beeinträchtigungen bei in vitro gezeugten gegenüber natürlich gezeugten Kindern vor (Chen und Heilbronn 2017). In einem systematischen Review wurde der Zusammenhang zwischen In-vitro-Befruchtung und langfristiger kognitiver Entwicklung mit speziellem Fokus auf die Qualität der Studien untersucht (Rumbold et al. 2017). Von den 35 zwischen 1995 und 2016 in 14 Ländern erstellten Studien wiesen sieben eine hohe, 20 eine mässige und acht eine schlechte Qualität auf. Die Autoren gelangen zum Schluss, dass in den qualitativ guten Studien keine Unterschiede in der kognitiven Entwicklung von natürlich und mittels In-vitro-Fertilisation (IVF) gezeugten Kindern festgestellt werden konnten.

Die Befunde zum Einfluss der Befruchtung mittels intrazytoplasmatischer Spermieninjektion (ICSI) auf die kognitive Entwicklung der Kinder sind hingegen widersprüchlich. In den Studien wurde einerseits die kognitive Entwicklung von mittels ICSI gezeugten und natürlich gezeugten Kindern verglichen. Von den drei qualitativ guten Studien zeigte eine Studie auf, dass der Intelligenzquotient 
(IQ) von in vitro gezeugten Kindern fünf bis sieben Punkte tiefer ist als der IQ von natürlich gezeugten Kindern (Knoester et al. 2008, zitiert in Rumbold et al. 2017). Zwei andere gute Studien zeigten hingegen keine signifikanten Unterschiede in der kognitiven Entwicklung von in vitro und natürlich gezeugten Kindern (Leslie et al. 2003; Bay et al. 2013, zitiert in Rumbold et al. 2017). Andererseits wurde die kognitive Entwicklung von Kindern nach IVF und ICSI verglichen. In einer qualitativ guten Studie wurde im Vergleich zur IVF ein um 50 Prozent erhöhtes Risiko einer geistigen Behinderung nach einer ICSI festgestellt (Sandin et al. 2013, zitiert in Rumbold et al. 2017). Zwei qualitativ gute Studien konnten hingegen keine Unterschiede zwischen den beiden Befruchtungsmethoden feststellen (Knoester et al. 2008; Leslie et al. 2003, zitiert in Rumbold et al. 2017). In einem anderen systematischen Review, das die langfristige Entwicklung von Kindern nach IVF und ICSI verglich, gelangten die Autoren zum Schluss, dass bei ICSI-Kindern möglicherweise ein erhöhtes Risiko für Autismus und intellektuelle Beeinträchtigungen besteht (Catford et al. 2017).

Die meisten bisherigen Studien sind auf Kinder beschränkt. Es fehlen Studien zur kognitiven Entwicklung nach einer In-vitro-Befruchtung bei Jugendlichen und jungen Erwachsenen (Rumbold et al. 2017, Catford et al. 2017). In einer gross angelegten Studie in Dänemark wurden die schulischen Leistungen von 15- bis 16-jährigen in vitro gezeugten Kindern untersucht und mit denjenigen von natürlich gezeugten Kindern verglichen. Dafür wurden Daten aus verschiedenen nationalen Datenbanken verknüpft (IVF-Register, dänisches Geburtenregister, Daten zu den Resultaten eines landesweiten Schultests sowie soziodemografische Daten des dänischen Statistikamtes). Die schulischen Leistungen der in vitro gezeugten 4766 Kinder fielen besser aus als diejenigen der zufällig ausgewählten natüllich gezeugten 5660 Kinder. Wenn jedoch der sozioökonomische Hintergrund der Eltern in der Berechnung berücksichtigt wurde, waren die schulischen Leistungen der in vitro gezeugten Kinder leicht schwächer. Hingegen zeigten sich keine Unterschiede in den schulischen Leistungen von mittels IVF und ICSI gezeugten Kindern (Spangmose et al. 2017).

Die wichtigste Schwäche der bisherigen Studien zur kognitiven Entwicklung der mittels In-vitro-Befruchtung gezeugten Kinder betrifft den Ausschluss von Kindern, die zu früh geboren wurden, ein tiefes Geburtsgewicht aufwiesen oder aus Mehrlingsgeburten stammten. Diese Kinder weisen ein erhöhtes Risiko einer eingeschränkten Lernfähigkeit und geistiger Behinderung auf (Rumbold et al. 2017). Da Frühgeburten, tiefes Geburtsgewicht und Mehrlingsschwanger- 
schaften nach einer In-vitro-Befruchtung häufiger auftreten, wird der negative Einfluss dieser Zeugungsmethode auf die kognitive Entwicklung von Kindern in den Studien möglicherweise unterschätzt (Spangmose et al. 2017).

\section{Ursachen der Risiken für das Kind}

Zum jetzigen Zeitpunkt können die Ursachen der oben beschriebenen Risiken für das Kind nicht abschliessend beurteilt werden. Es ist unklar, ob diese Risiken auf die ovarielle Stimulation und die In-vitro-Befruchtung, die eingeschränkte Fruchtbarkeit (Subfertilität) der Eltern oder eine Kombination der beiden Ursachen zurückzuführen sind. Studien mit widersprüchlichen Ergebnissen finden sich zu allen diskutierten Risiken (Chen und Heilbronn 2017, Qin et al. 2017b, Seegers et al. 2016, Fauser et al. 2014, Rumbold et al. 2017, Ziru et al. 2017).

Auf die beschriebenen perinatalen Risiken scheint sowohl die Technologie als auch die Subfertilität einen Einfluss zu haben (Chen und Heilbronn 2017, Qin et al. 2017b). In einer retrospektiven Kohortenstudie in einer niederländischen Klinik wurde aufgezeigt, dass Kinder aus In-vitro-Befruchtung von 19'840 Frauen ein tieferes Geburtsgewicht hatten und früher geboren wurden als natürlich gezeugte Kinder von 6588 subfertilen Frauen (Ceelen et al. 2008, zitiert in Fauser et al. 2014). Eine weitere retrospektive Kohortenstudie mit 1246 fruchtbaren und 461 unfruchtbaren Frauen kam dagegen zum Schluss, dass sich vor allem die eingeschränkte Fruchtbarkeit negativ auf die fötale Entwicklung auswirkt (Cooper et al. 2011, zitiert in Fauser et al. 2014). Eine auf nationalen Registerdaten basierende niederländische Studie mit 1813 Geschwisterpaaren, von denen eines in vitro und das andere natürlich gezeugt wurde, gelangt zum Schluss, dass die mütterliche Subfertilität und nicht die In-vitro-Befruchtung für das tiefe Geburtsgewicht verantwortlich ist (Seggers et al. 2016). Das erhöhte Risiko von angeborenen Fehlbildungen lässt sich zumindest teilweise auf die eingeschränkte Fruchtbarkeit der Eltern zurückführen und könnte durch die ovarielle Stimulation sowie die In-vitro-Befruchtung weiter erhöht werden (Chen und Heilbronn 2017).

Weniger klare Aussagen können zum Zusammenhang zwischen Subfertilität, Technologie und längerfristigen Risiken gemacht werden. Zurzeit scheint es unklar, ob die Subfertilität oder die Reproduktionsmedizin für das erhöhte Krebsrisiko ausschlaggebend ist. Auch bezüglich der kognitiven Entwicklung 
lässt sich aufgrund der methodologischen Schwächen der bisherigen Studien nicht abschliessend beurteilen, ob die Technologie oder die Subfertilität die entscheidende Einflussgrösse ist (Rumbold et al. 2017). Die ovarielle Stimulation, die In-vitro-Befruchtung und die Subfertilität beeinflussen wahrscheinlich alle die beobachteten kardiovaskulären Risiken bei Kindern. Um die relative Bedeutung der verschiedenen Einflussfaktoren zu klären, braucht es nach den wegweisenden präklinischen Untersuchungen und kleinen Kohortenstudien nun gross angelegte, gut kontrollierte epidemiologische Studien (Chen und Heilbronn 2017). Gemäss anderen Autoren zeigen Geschwisterstudien deutlich, dass primär die reproduktionsmedizinischen Verfahren zu Herz-KreislaufErkrankungen führen und diese nicht ausschliesslich auf die elterliche Unfruchtbarkeit zurückgeführt werden können (Fleming et al. 2018).

Wie die Technologie die gesundheitlichen Risiken für die Kinder beeinflussen könnte, war lange Zeit unklar. Das relativ junge Forschungsgebiet der Epigenetik liefert neue Hinweise auf einen Zusammenhang zwischen reproduktionsmedizinischen Verfahren und gesundheitlichen Risiken bei Kindern. Die Epigenetik untersucht Schaltmechanismen, die Gene ein- oder ausschalten, ohne die Gene selbst zu verändern. In Zusammenhang mit der In-vitro-Befruchtung wird vor allem ein solcher Schaltmechanismus - die DNA-Methylierung - diskutiert (Lazaraviciute et al. 2014). Die DNA-Methylierung respektive die DNA-Demethylierung findet zu zwei Zeitpunkten statt: einerseits bei der Bildung der Eizellen und andererseits vor der Einnistung des Embryos in der Gebärmutter (Chen und Heilbronn 2017). Es ist deshalb naheliegend, dass die Hormonstimulation der Eierstöcke, die Eizellentnahme, die Manipulierung der Spermien oder die für die Ausreifung der Embryonen benötigten Nährmedien zu epigenetischen Veränderungen führen könnten (Lazaraviciute et al. 2014, Fauser et al. 2014, Ziru et al. 2017).

Relativ gut nachgewiesen ist ein Zusammenhang zwischen In-vitro-Befruchtung und Erkrankungen, die durch genomische Prägung (Genomic Imprinting) verursacht werden (Fauser et al. 2014, Lazaraviciute et al. 2014). Davon spricht man, wenn nur die mütterlichen oder väterlichen Erbinformationen in einem Gen ausgeschaltet werden. Dazu zählen das Beckwith-Wiedemann-Syndrom (BWS) und das Angelman-Syndrom (AS). Gemäss einem Literatur-Review hatten 90 bis 100 Prozent der in vitro gezeugten Kinder mit einem BeckwithWiedemann-Syndrom eine genomische Prägung. Eine solche Prägung wurde hingegen nur bei 40 bis 50 Prozent der natürlich gezeugten Kinder mit dieser 
Erkrankung festgestellt. Beim Angelman-Syndrom waren 71 Prozent der in vitro gezeugten Kinder diesbezüglich auffällig, während es bei den natürlich gezeugten Kindern nur 5 Prozent waren (Manipalviratn et al. 2009, zitiert in Fauser et al. 2014). Allerdings tritt das Beckwith-Wiedemann-Syndrom bei weniger als einem Prozent der in vitro gezeugten Kinder auf (Fauser et al. 2014).

Auch die Entstehung von Krebs bei Menschen (Hargreave et al. 2013, zitiert in Catford et al. 2017) und die kognitive Entwicklung in Tiermodellen (Wu et al. 2014, zitiert in Rumbold et al. 2017) wurden bereits mit epigenetischen Veränderungen in Verbindung gebracht. Am weitesten fortgeschritten sind Untersuchungen zum Einfluss epigenetischer Veränderung auf die Entwicklung chronischer Erkrankungen wie Übergewicht, Diabetes, Bluthochdruck und HerzKreislauf-Erkrankungen. So wurden bei in vitro gezeugten Kindern Veränderungen in der DNA-Methylierung in Genen beobachtet, die unter anderem in Zusammenhang mit der Entstehung von Übergewicht und Diabetes stehen. Weiter scheinen Veränderungen bei der DNA-Methylierung gemäss verschiedenen Studien die Funktionsweise und Entwicklung der Plazenta zu beeinträchtigen. Die Folge davon können Schwangerschaftsvergiftungen und ein reduziertes Wachstum des Fötus im Bauch sein (Chen et al. 2017). Nach solchen Schwangerschaftskomplikationen weisen auch natürlich gezeugte Kinder Gefässfunktionsstörungen auf, die mit einem erhöhten Risiko von Herz-Kreislauf-Erkrankungen im Erwachsenenalter einhergehen (Scherrer et al. 2015). Zudem wurde in der Studie der Forschergruppe am Inselspital bei in vitro gezeugten Mäusen eine veränderte DNA-Methylierung in den für die Lungen- und Herzentwicklung verantwortlichen Genen beobachtet, die zu Gefässveränderungen in den betroffenen Organen führte. Entsprechende Vermutungen wurden auch in Studien bei Wiederkäuern gemacht (Fauser et al. 2014). Allerdings lassen sich diese Studien mit Tieren nur bedingt auf Menschen übertragen (Menezo et al. 2010, zitiert in Fauser et al. 2014).

\subsubsection{Zusätzliche Risiken durch Social Freezing}

Die im Kapitel 2.2.2 beschriebenen gesundheitlichen Risiken für in vitro gezeugte Kinder betreffen die Reproduktionsmedizin generell. Nachfolgend werden zusätzliche Risiken in Zusammenhang mit Social Freezing diskutiert. Dies betrifft einerseits die Technologie der Kryokonservierung der Eizellen und ande- 
rerseits das Alter der Frau bei der Befruchtung ihrer Eizellen in vitro. Zudem sind mit einer breiten Anwendung des Social Freezing Risiken für die öffentliche Gesundheit verbunden.

\section{Risiken durch die Kryokonservierung der Eizellen}

Bisher gibt es keine Hinweise darauf, dass die Kryokonservierung der Eizellen zu zusätzlichen Risiken für das Kind führt. So scheint die Fehlbildungsrate bei aus kryokonservierten Eizellen gezeugten Kindern nicht erhöht zu sein. In der Literatur werden dazu im Wesentlichen zwei Artikel zitiert. Ein Review mit 936 Kindern kam zum Schluss, dass die Fehlbildungsrate von 1,3 Prozent bei diesen Kindern nicht höher liegt als in der amerikanischen Bevölkerung (Noyes et al. 2008, zitiert in ASRM 2013, ESHR 2012, NEK 2017). Sie liegt auch unter der bei deutschen Kindern beobachteten Fehlbildungsrate von rund 3 Prozent (Queißer-Luft et al. 2009, zitiert in Bernstein und Wiesemann 2014). In einer Studie mit 200 Kindern aus vitrifizierten Eizellen wurden in Bezug auf Geburtsgewicht und Fehlbildungen ebenfalls keine Unterschiede zu Kindern gefunden, die mit frischen Eizellen im Rahmen einer Befruchtung in vitro gezeugt wurden (Chian et al. 2008, zitiert in ASRM 2013, ESHR 2012, NEK 2017).

Neuere Studien gelangen zu ähnlichen Befunden. In einer retrospektiven Kohortenstudie in einer spanischen Privatklinik wurden die perinatale Entwicklung und die Fehlbildungsrate bei 1027 Kindern aus vitrifizierten Eizellen und 1224 Kindern aus frischen Eizellen verglichen (Cabo et al. 2014). In der Gruppe der Kinder aus vitrifizierten Eizellen betrug die Fehlbildungsrate 1,7 Prozent, wovon 0,7 Prozent auf schwere und 1,0 Prozent auf leichtere Fehlbildungen fielen. Allerdings stammten sowohl die frischen als auch die vitrifizierten Eizellen mehrheitlich von jungen Eizellspenderinnen. In einer weiteren Studie in Italien wurden lediglich die Ergebnisse bei Kindern aus eigenen Eizellen untersucht, die entweder langsam eingefroren oder vitrifiziert wurden. Grundlage bildeten die zwischen 2005 und 2013 erhobenen Daten im nationalen Register zur Reproduktionsmedizin. Von den 2152 in diesem Zeitraum aus eingefrorenen Eizellen gezeugten Kindern wiesen lediglich 20 schwere Fehlbildungen auf (0,9 Prozent). Da die Erfassung von Fehlbildungen im italienischen Register nicht obligatorisch ist, dürfte die Fehlbildungsrate in Wirklichkeit aber höher liegen (Levi-Setti et al. 2016). 
Als Ursache für allfällige Fehlentwicklungen bei Kindern aus kryokonservierten Eizellen stehen Veränderungen der meiotischen Spindel im Fokus. Die meiotische Spindel sorgt für die richtige Ausrichtung und Trennung der Chromosomen in der Eizelle. Sie reagiert sehr sensibel auf Veränderungen in der Temperatur sowie die im Rahmen der Kryokonservierung eingesetzte kryoprotektive Lösung. In einzelnen Studien wurden nach der Ausreifung der kryokonservierten Eizellen in der Glasschale veränderte Spindelstrukturen und Chromosomenanomalien beobachtet (Park et al. 1997, zitiert in Fauser et al. 2014). In mehreren Studien wurde jedoch berichtet, dass die Spindel nach dem Auftauen zu ihrer normalen Konfiguration zurückfindet (Bianchi et al. 2005; Cobo et al. 2001; Gook et al. 1994: Rienzi et al. 2004; Stachecki et al. 2004, zitiert in Fauser et al. 2014). Je nach Alter der Frau wirkt sich die Vitrifikation allerdings unterschiedlich auf die Spindelkonfiguration und die Chromosomenanordnung aus. So wurden bei Frauen mit einem medianen Alter von 25,1 Jahren drei Stunden nach dem Auftauen der Eizellen keine Auffälligkeiten entdeckt. Bei einem medianen Alter von 38,5 Jahren war jedoch nur noch knapp ein Drittel der Eizellen unauffällig (Nawroth 2015).

Die Lagerungsdauer scheint keinen entscheidenden Einfluss zu haben. Allerdings sind die Auswirkungen einer längeren Lagerungsdauer bisher noch wenig untersucht. Die ASRM identifizierte lediglich eine Studie, die zwischen nach 48 Monaten sowie früher aufgetauten Eizellen keine Unterschiede in der Überlebens-, Befruchtungs-, Einnistungs- und Geburtenrate finden konnte (Parmegiani et al. 2009, zitiert in ASRM 2013). Gemäss Nawroth (2015) ist der Einfrierund Auftauprozess der Eizellen entscheidend, während die Lagerungsdauer keine Rolle zu spielen scheint. So wurden Kinder aus Eizellen geboren, die zwölf Jahre gelagert worden waren.

Sowohl die amerikanische als auch die europäische Fachgesellschaft der Reproduktionsmediziner sind der Ansicht, dass diese ersten Befunde zur Kryokonservierung von Eizellen beruhigend sind. Sie vertreten jedoch ebenfalls die Meinung, dass zusätzliche Daten insbesondere zu den langfristigen Folgen der Kryokonservierung für die Gesundheit der Kinder nötig sind (ASRM 2013, ESHR 2012). 
Unabhängig von der Technologie bildet das Alter der Frau bei der Befruchtung in vitro einen Risikofaktor. Beim Social Freezing lässt eine Frau ihre Eizellen in der Regel in bereits fortgeschrittenem Alter in vitro befruchten. Die Risiken in Zusammenhang mit Schwangerschaft und Geburt steigen jedoch mit dem Alter der Mutter an. Bisher haben nur wenige Frauen auf ihre kryokonservierten Eizellen zurückgegriffen, sodass zurzeit wenige Informationen zum Schwangerschaftsverlauf bei solchen Frauen vorliegen. Die Verwendung von in jungem Alter kryokonservierten Eizellen kann den Schwangerschaftsverlauf insofern beeinflussen, als das Risiko früher Fehlgeburten sinkt. Andere Schwangerschaftskomplikationen sind in erster Linie abhängig vom Gesundheitszustand der Frau (Wunder 2014). Nachfolgend werden die Risiken für Mutter und Kind bei Frauen zusammengefasst, die nach natürlicher Zeugung oder Eizellspende schwanger geworden sind. Dabei wird zwischen drei Altersgruppen mit unterschiedlichem Risikoprofil unterschieden.

40 bis 44 Jahre: Grössere Studien zu den Risiken einer Schwangerschaft Anfang 40 sind zu beruhigenden Resultaten gelangt (Belaisch-Allart et al. 2013). Zwar nehmen in diesem Alter die Schwangerschaftsrisiken zu. So steigt mit zunehmendem Alter das Risiko vorbestehender Krankheiten wie Bluthochdruck, Diabetes und Herzprobleme und damit das Risiko von Komplikationen während der Schwangerschaft und der Geburt (Belaisch-Allart et al. 2013, Wunder et al. 2014). Auch treten mit zunehmendem Alter mehr Schwangerschaftskomplikationen (Plazenta praevia, Blutungen im dritten Trimester, Schwangerschaftsdiabetes) auf und der Anteil an Kaiserschnitten steigt. Schliesslich werden mehr Kinder zu früh respektive mit einem zu niedrigen Geburtsgewicht geboren. Insgesamt sind jedoch 81,6 Prozent der Schwangerschaften in der Altersgruppe von 40 bis 44 Jahren mit einem tiefen Risiko verbunden. In diesen Zahlen sind sowohl Schwangerschaften nach einer natürlichen Zeugung als auch nach einer Befruchtung der Eizellen in vitro eingeschlossen, weil im Datensatz mit über acht Millionen Geburten aus einer amerikanischen Kohortenstudie nicht nach der Zeugungsmethode unterschieden wurde (Luke und Brown 2007, zitiert in Belaisch-Allart et al. 2013).

45 bis 50 Jahre: Die Schwangerschaften ab Mitte 40 sind mit einem moderaten Risiko für die Kinder und einem erhöhten Risiko für ihre Mütter verbunden (Belaisch-Allart et al. 2013). Die meisten Daten zu Schwangerschaften in die- 
sem Alter stammen aus Eizellspendeprogrammen. Da eine Eizellspende zu zusätzlichen Schwangerschaftskomplikationen führen kann (Blázquez et al. 2016, Masoudian et al. 2016), können die Resultate nicht direkt auf Frauen übertragen werden, die dank eigener kryokonservierter Eizellen schwanger werden. Publikationen zu spontanen Schwangerschaften sind selten. Eine Studie mit 131 Erstgebärenden über 45 Jahre in Israel zeigte, dass diese im Vergleich zu jüngeren Frauen in der Schwangerschaft häufiger unter Bluthochdruck (45\% versus $6,4 \%)$, Schwangerschaftsdiabetes $(42,7 \%$ versus $6,1 \%)$, Schwangerschaftsvergiftung (18,3\% versus $4 \%$ ), Frühgeburten (33\%) leiden und die Geburt meistens mittels Kaiserschnitt (93,9\%) erfolgt (Glasser et al. 2011, zitiert in Wunder 2014).

Ab 50 Jahren: Ab 50 Jahren sind Schwangerschaften sowohl für die Mutter als auch für die Kinder mit einem grossen Risiko verbunden (Wunder 2014, Belaisch-Allart et al. 2013). Im Vergleich zu Frauen zwischen 40 und 49 Jahren treten bei über 50-jährigen Frauen signifikant häufiger Herzprobleme, Bluthochdruck, Diabetes und Schwangerschaftsvergiftung auf. Die Kinder von über 50Jährigen werden früher geboren und haben ein tieferes Geburtsgewicht als Kinder von jüngeren Frauen (Wunder 2014). Die entsprechenden Daten stammen in der Regel aus Studien zu Schwangerschaften nach Eizellspende (Belaisch-Allart et al. 2013).

Gestützt auf diese Befunde wird in der Literatur empfohlen, keine Schwangerschaft über 50 Jahren anzustreben und zwischen 45 und 50 Jahren nur bei gesunden Frauen nach Vorliegen eines geburtshilflichen und psychologischsozialen Gutachtens (Wunder 2014).

Risiken für die öffentliche Gesundheit bei einer breiten Anwendung des Social Freezing

Im Gegensatz zur Anwendung reproduktionsmedizinischer Technologien bei unfruchtbaren Paaren, die nur einer medizinisch umschriebenen Population angeboten werden, steht Social Freezing grundsätzlich allen Frauen zur Verfügung. Deshalb erhält der Public-Health-Aspekt der mit der Technologie verbundenen gesundheitlichen Risiken besondere Bedeutung. 
Die verschiedenen epidemiologischen Studien und Tierversuchsmodelle liefern klare Hinweise darauf, dass bleibende Erhöhungen kardiovaskulärer Risikomarker aufgrund epigenetischer Veränderungen als direkte Folgen der Technologie häufig sein könnten. Diese Ergebnisse sind noch relativ neu und müssen bestätigt werden, sie wurden aber bisher auch nicht durch vergleichbare Untersuchungen widerlegt. Weitere Studien sind insbesondere notwendig, um zu klären, ob die epigenetischen Veränderungen auf die Technologie, die Subfertilität der Eltern oder eine Kombination von beidem zurückzuführen sind (Fauser et al. 2014., Catford et al. 2017, Lazaraviciute et al. 2014, Rumbold et al. 2017, Vermeiden und Bernardus 2013, Scherrer et al. 2015, Chen und Heilbronn 2017). Unbedenklich wären reproduktionsmedizinische Verfahren für fruchtbare Frauen, die ihre Eizellen aus sozialen Gründen einfrieren lassen, nur dann, wenn die damit verbundenen gesundheitlichen Risiken für die Kinder ausschliesslich auf die Subfertilität der Eltern zurückzuführen wären. Wenn dagegen die Technologie direkt mit einem deutlich erhöhten Risiko von HerzKreislauf-Erkrankungen verbunden wäre, würde jede weitere Verbreitung des Social Freezing zu einem proportionalen Anstieg der nicht übertragbaren Krankheiten (NCDs) in der Bevölkerung führen und mit bedeutsamen Auswirkungen auf die öffentliche Gesundheit verbunden sein, mindestens so lange, als das erhöhte Risiko nicht durch weitere technologische Fortschritte minimiert werden könnte. Da sich die Laborbedingungen in der Reproduktionsmedizin in den letzten zehn bis 15 Jahren stark verändert haben, ist unklar, ob die heute in vitro gezeugten Kinder nach wie vor den gleichen Gesundheitsrisiken ausgesetzt sind wie die Kinder, die vor einigen Jahren in vitro gezeugt wurden und deren Gesundheit gegenwärtig im Rahmen von Studien untersucht wird.

\subsection{Erfolgsaussichten}

In Zusammenhang mit Social Freezing werden insbesondere zwei Parameter für den Erfolg des Social Freezing diskutiert. Einerseits werden die Erfolgsaussichten dieser Technologie mit alternativen reproduktionsmedizinischen Verfahren verglichen. Dazu zählt die Verwendung von frischen Eizellen in fortgeschrittenem Alter respektive eine Eizellspende. Andererseits ist es für die betroffenen Frauen wichtig, die theoretische Chance auf eine Lebendgeburt (Baby-TakeHome-Rate) zu kennen, bevor sie sich für die Technologie entscheiden. 


\subsection{1 \\ Erfolgsaussichten im Vergleich zu anderen reproduktions- medizinischen Verfahren}

Die Erfolgsgeschichte der Vitrifikation ist darauf zurückzuführen, dass sich die Befruchtungs- und Schwangerschaftsraten nach der In-vitro-Befruchtung von eingefrorenen Eizellen kaum mehr von denjenigen mit frischen Eizellen unterscheiden (Wunder 2014). Dieser Befund stützt sich insbesondere auf vier randomisierte kontrollierte Studien, welche die Ergebnisse mit kryokonservierten und frischen Eizellen verglichen. Zwei dieser Studien wurden in Spanien, basierend auf gespendeten Eizellen, durchgeführt (Cobo 2008; Cobo 2010, zitiert in ASRM 2013). Die beiden anderen Studien wurden in Italien realisiert, wo die Kryokonservierung von befruchteten Eizellen lange verboten war und nur unbefruchtete Eizellen eingefroren werden konnten. Die italienischen randomisierten kontrollierten Studien beruhen darum auf überzähligen Eizellen von unfruchtbaren Paaren (Rienzi 2010; Parmegiani 2011, zitiert in ASRM 2013). Diese vier Studien zeigen, dass zwischen 90 und 97 Prozent der Eizellen das Auftauen überleben, sich danach 71 bis 79 Prozent befruchten lassen und sich schliesslich 17 bis 41 Prozent in der Gebärmutter einnisten. Die klinische Schwangerschaftsrate beläuft sich in diesen vier Studien auf 36 bis 61 Prozent pro Transfer von Eizellen (ASRM 2013). Randomisierte kontrollierte Studien liefern in der medizinischen Forschung zwar die aussagekräftigsten Ergebnisse. Gemäss der amerikanischen Fachgesellschaft für Reproduktionsmedizin (ASRM 2013) können die Ergebnisse aufgrund von lediglich vier Studien jedoch nicht verallgemeinert werden. Sie äussert zwei Vorbehalte:

- Übertragbarkeit der Ergebnisse auf andere Kliniken: Die ASRM ist der Ansicht, dass solche Studien eher in erfolgreichen Kliniken mit hohen Schwangerschaftsraten durchgeführt und die Ergebnisse nicht ohne Weiteres auf andere Kliniken übertragen werden können. Aus diesem Grund hat die ASRM auch Beobachtungsstudien aus Italien in ihre Beurteilung der Kryokonservierung von Eizellen eingeschlossen. Diese Daten reflektieren die Erfahrung einer grossen Bandbreite von Institutionen und nicht nur die Expertise einzelner hochspezialisierter Kliniken. Sowohl eine multizentrische prospektive Kohortenstudie (Borini et al. 2010, zitiert in ASRM 2013) als auch eine Auswertung von nationalen Registerdaten (Scaravelli et al. 2010, zitiert in ASRM 2013) gelangen zum Schluss, dass Einnistungs- und Schwangerschaftsraten mit frischen Eizellen besser sind als mit kryokonservierten Eizellen. Diese Ergebnisse drücken möglicherweise eine Ver- 
zerrung bei der Auswahl der Eizellen aus. So werden in Italien aufgrund der gesetzlichen Rahmenbedingungen zuerst qualitativ gute Eizellen befruchtet und alle übrigen Eizellen eingefroren. Die Ergebnisse könnten aber auch Ausdruck des unterschiedlichen Erfahrungshintergrundes der Kliniken sein (ASRM 2013).

- Übertragbarkeit der Ergebnisse auf andere Patientenpopulationen: Gemäss der ASRM stammen die meisten Daten in den randomisierten kontrollierten Studien aus spanischen Eizellspendeprogrammen. Die ASRM bezweifelt, dass diese Daten von jungen fruchtbaren Eizellspenderinnen auf ältere Frauen übertragen werden können, die zurzeit hauptsächlich ihre Eizellen kryokonservieren lassen. Diese Zweifel scheinen nicht unberechtigt, wie eine Metaanalyse von 17 Studien zur Vitrifikation von Eizellen zeigt (Potadar et al. 2014, zitiert in Nawroth 2015). Diese Metaanalyse zeigte zwar keine signifikanten Unterschiede in der fortlaufenden Schwangerschaftsrate von frischen und vitrifizierten Eizellen auf. Auch in Studien, die ausschliesslich auf Spenderprogrammen beruhen, war der Unterschied nicht signifikant. Als jedoch Studien ohne Eizellspende eingeschlossen wurden, war bei vitrifizierten Eizellen eine signifikante Reduktion der fortlaufenden Schwangerschaftsrate zu beobachten. Die Autoren der Metaanalyse vermuten, dass diese Beobachtung darauf zurückzuführen ist, dass bei unfruchtbaren Paaren das Durchschnittsalter der Frauen, denen die Eizellen entnommen werden, höher ist als dasjenige von Eizellspenderinnen.

Im Vergleich zu einer In-vitro-Befruchtung mit frischen Eizellen in fortgeschrittenem Alter scheinen die Vorteile einer Befruchtung von in jungem Alter entnommenen und vitrifizierten Eizellen allerdings zu überwiegen. Wenn Frauen nicht auf eigene eingefrorene Eizellen zurückgreifen können und erst mit über 40 Jahren eine In-vitro-Befruchtung anstreben, weisen ihre Eizellen mehr Chromosomenanomalien auf und es gibt mehr Fehlgeburten (Académie nationale de médecine 2017, Belaisch-Allart et al. 2013). Social Freezing ist zudem möglicherweise kosteneffektiver als eine Befruchtung mit frischen, in fortgeschrittenem Alter entnommenen Eizellen. Allerdings gelangen drei auf einem Markov-Modell basierende Studien zur Kosteneffektivität des Social Freezing zu unterschiedlichen Resultaten. In einer amerikanischen Studie war die In-vitroBefruchtung von im Alter von 35 Jahren eingefrorenen Eizellen deutlich kosteneffektiver als eine klassische IVF bei 40-Jährigen (Devine et al. 2015). Da die 
Kosten für die Reproduktionsmedizin in den USA wesentlich höher sind als in Europa, können diese Schlussfolgerungen nicht ohne Weiteres auf die Schweiz übertragen werden. In einer deutschen Studie wurde die Kosteneffektivität des Social Freezing negativer beurteilt. Die Autoren folgern, dass zwei bis drei Zyklen Eizellkonservierung zwar zu einer geringfügig höheren Lebendgeburtenrate führen, gleichzeitig aber höhere Kosten verursachen, als wenn eine Frau mit 40 zuerst während eines Jahres eine Spontankonzeption anstrebt und nach einem Jahr drei klassische IVF-Zyklen durchläuft (Klüber et al. 2016). In einer niederländischen Studie gelangten die Autoren zum Schluss, dass Social Freezing kosteneffektiver ist als eine klassische IVF ab 40 Jahren, wenn 61 Prozent der Frauen, die im Alter von 35 Jahren Eizellen eingefroren haben, diese in vitro befruchten lassen (Loendersloot et al. 2011).

Social Freezing wird aufgrund medizinischer Vorteile allerdings auch als Alternative zur Eizellspende diskutiert. Aufgrund von Problemen bei der Immuntoleranz führt die Eizellspende zu einem erhöhten Risiko von Schwangerschaftsund Geburtskomplikationen wie Schwangerschaftsvergiftung (Blázquez et al. 2016; Masoudian et al. 2016).

\subsubsection{Individuelle Erfolgsaussichten}

Für Frauen, die ihre Eizellen kryokonservieren lassen, ist es von Bedeutung, die theoretische Chance auf ein Kind dank dieser Technologie zu kennen. In vier Studien wurde die theoretische Geburtenchance je nach Alter bei der Eizellentnahme und der Anzahl eingefrorener Eizellen berechnet (vgl. Tabelle 2).

Diese Studien beruhen auf Daten aus einer spanischen Klinik (Cobo et al. 2016), zwei amerikanischen Kliniken (Doyle et al. 2016, Goldman et al. 2017) sowie aus dem in deutschsprachigen Ländern angesiedelten FertiPROTEKTRegister (von Wolff et al. 2015). Diese Studien liefern Informationen, wie sich das Alter bei der Eizellentnahme und die Anzahl eingefrorener Eizellen auf die theoretische Geburtenchance auswirken. 
Tabelle 2: $\quad$ Erfolgsaussichten des Social Freezing

\begin{tabular}{|c|c|c|c|c|}
\hline $\begin{array}{l}\text { Alter } \\
\text { bei Eizell- } \\
\text { entnahme }\end{array}$ & $\begin{array}{l}\text { Anzahl } \\
\text { eingefrorene } \\
\text { Eizellen }\end{array}$ & $\begin{array}{l}\text { Theo- } \\
\text { retische } \\
\text { Geburten- } \\
\text { chance }\end{array}$ & Grundgesamtheit & Quelle \\
\hline $\begin{array}{l}\leq 35 \text { Jahre } \\
\geq 36 \text { Jahre }\end{array}$ & $\begin{array}{l}15 \text { Eizellen } \\
11 \text { Eizellen }\end{array}$ & $\begin{array}{l}85,2 \% \\
35,6 \%\end{array}$ & $\begin{array}{l}191 \text { Zyklen bei } 137 \text { Frauen, } \\
\text { wovon } 17 \text { unter einer beein- } \\
\text { trächtigten Fruchtbarkeit } \\
\text { litten }\end{array}$ & $\begin{array}{l}\text { Cobo } \\
\text { et al. } 2016\end{array}$ \\
\hline $\begin{array}{l}\leq 38 \text { Jahre } \\
38-40 \text { Jahre }\end{array}$ & $\begin{array}{l}\text { 15-20 Eizellen } \\
\text { 25-30 Eizellen }\end{array}$ & $\begin{array}{l}70-80 \% \\
65-75 \%\end{array}$ & $\begin{array}{l}128 \text { Zyklen mit eingefrore- } \\
\text { nen Eizellen bei } 117 \text { Frauen } \\
\text { - bei } 31 \text { aus sozialen Grün- } \\
\text { den - sowie } 18 \text { '746 Zyklen } \\
\text { mit frischen Eizellen }\end{array}$ & $\begin{array}{l}\text { Doyle } \\
\text { et al. } 2016\end{array}$ \\
\hline $\begin{array}{l}34 \text { Jahre } \\
37 \text { Jahre } \\
42 \text { Jahre }\end{array}$ & $\begin{array}{l}20 \text { Eizellen } \\
20 \text { Eizellen } \\
20 \text { Eizellen }(61)^{1}\end{array}$ & $\begin{array}{l}90 \% \\
75 \% \\
37 \% \\
(75 \%)^{1}\end{array}$ & $\begin{array}{l}466 \text { Zyklen, davon } 423 \text { bei } \\
\text { männlicher Unfruchtbarkeit, } \\
43 \text { bei Eileiterproblemen }\end{array}$ & $\begin{array}{l}\text { Goldman } \\
\text { et al. } 2017\end{array}$ \\
\hline $\begin{array}{l}<35 \text { Jahre } \\
\text { 35-39 Jahre } \\
\text { 40-44 Jahre }\end{array}$ & $\begin{array}{l}\sim 11 \text { Eizellen }(\sim 33)^{2} \\
\sim 11 \text { Eizellen } \\
\sim 10 \text { Eizellen }\end{array}$ & $\begin{array}{l}40 \% \\
(65 \%)^{2} \\
30 \% \\
15 \%\end{array}$ & 134 Frauen & $\begin{array}{l}\text { Wolff } \\
\text { et al. } 2015\end{array}$ \\
\hline
\end{tabular}

Legende: SF $=$ Social Freezing, ${ }^{1}=$ Um eine theoretische Geburtenchance von $75 \%$ zu erreichen, müssten im Alter von 42 Jahren 61 Eizellen entnommen werden, ${ }^{2}=\operatorname{Im}$ Schnitt werden bei Frauen unter 35 Jahren pro Stimulationszyklus 11 Eizellen entnommen. Für eine theoretische Geburtenchance von 65 \% müssten drei Zyklen mit rund 33 Eizellen durchgeführt werden (von Wolff 2013). 


\section{Alter bei der Entnahme von Eizellen}

Da sich Frauen in der Regel erst mit Mitte 30 für Social Freezing interessieren (vgl. Kapitel 3.1.2), sind sie auf verlässliche Angaben zur theoretischen Geburtenchance in diesem Alter angewiesen. Alle vier aufgeführten Studien zeigen zwar einhellig, dass die theoretische Geburtenchance mit steigendem Alter sinkt. Dies ist darauf zurückzuführen, dass die Qualität der Eizellen mit zunehmendem Alter abnimmt. In Eizellen von älteren Frauen treten mehr Chromosomenstörungen auf, die sich in abnehmenden Implantationsraten und ansteigenden Fehlgeburtsraten manifestieren (Van der Ven 2017). Die aufgeführten Studien gelangen jedoch zu einer unterschiedlichen Einschätzung bezüglich des Alters, ab dem die theoretische Geburtenchance stark sinkt. Mit 15 bis 20 Eizellen besteht in der spanischen Studie (Cabo et al. 2016) lediglich bei unter 35-Jährigen eine gute theoretische Geburtenchance (85,2 \%), während in den amerikanischen Studien (Doyle et al. 2016, Goldman et al. 2017) ein vergleichbares Ergebnis (70-80 \%) bis zum 38. Geburtstag erreicht werden kann.

\section{Anzahl einzufrierender Eizellen}

Im Rahmen des Social Freezing sollen einerseits genügend Eizellen entnommen werden, um eine möglichst gute theoretische Geburtenchance zu gewährleisten. Andererseits sollen sich die Frauen nicht unnötigen Stimulationszyklen unterziehen und zu viel für die Behandlung ausgeben müssen. Aus diesem Grund sind genaue Kenntnisse über die Anzahl notwendiger Eizellen pro Altersgruppe von Bedeutung. In den vier Studien besteht allerdings Uneinigkeit darüber, ab welchem Alter und ab welcher Anzahl Eizellen die Kryokonservierung zusätzlicher Eizellen keinen Nutzen mehr bringt. In der spanischen Studie steigt die theoretische Geburtenchance bei unter 35-jährigen Frauen mit jeder aufgetauten und befruchteten Eizelle beträchtlich an. Ab zehn Eizellen flacht die Kurve allerdings ab und erreicht bei 15 Eizellen eine Obergrenze mit einer theoretischen Geburtenchance von 85,2 Prozent. Bei über 36-jährigen Frauen wird diese Obergrenze bei elf Eizellen und einer theoretischen Geburtenchance von 35,6 Prozent erreicht. Diese theoretische Geburtenchance kann durch die Kryokonservierung von mehr als elf Eizellen nicht weiter verbessert werden (Cabo et al. 2016). Im FertiPROTEKT-Register wurde eine maximale theoretische Geburtenchance nach drei Stimulationszyklen von 65 Prozent bei unter 35jährigen Frauen berechnet (von Wolff 2013). Die amerikanischen Studien gehen 
hingegen davon aus, dass auch bei über 35-jährigen Frauen dank zusätzlicher Eizellen die theoretische Geburtenchance verbessert werden kann. Für eine theoretische Geburtenchance von 65 bis 75 Prozent sind bei 38- bis 40-jährigen Frauen 25 bis 30 Eizellen notwendig (Doyle et al. 2016). Für eine vergleichbare theoretische Geburtenchance müssten im Alter von 42 Jahren 61 Eizellen eingefroren werden. Dafür wären bei einer durchschnittlichen Eizellentnahme von 10,3 Eizellen im Alter von 42 Jahren allerdings sechs Stimulationszyklen notwendig (Goldman et al. 2017).

Diese Unterschiede zwischen den vier Studien sind auf drei Ursachen zurückzuführen:

- Erstens beruhen die Berechnungen in den spanischen (Cabo et al. 2016) und amerikanischen Studien (Doyle et al. 2016, Goldman et al. 2017) auf eigenen klinischen Daten zum Anteil der Eizellen, die sich erfolgreich auftauen und befruchten liessen und danach zu klinischen Schwangerschaften respektive Geburten führten. Da im FertiPROTEKT-Register keine solchen Daten erhoben werden konnten, beruhen die Berechnungen der theoretischen Geburtenchance auf entsprechenden Daten aus Italien (von Wolff et al. 2015). Aufgrund unterschiedlicher klinischer Daten können die Berechnungen der theoretischen Geburtenchance in diesen Studien nicht ohne Weiteres auf andere reproduktionsmedizinische Kliniken übertragen werden. Goldman et al. (2016) empfehlen anderen Reproduktionsmedizinerinnen und -mediziner, ihr Modell zur Berechnung der theoretischen Geburtenchance, gestützt auf eigene klinische Daten, anzupassen.

- Zweitens beruhen die Berechnungen auf unterschiedlichen Patientenkollektiven. Die spanische Studie beruht als einzige ausschliesslich auf Daten von Frauen, die ihre Eizellen aus sozialen Gründen kryokonservieren liessen. Allerdings ist es bei einer Grundgesamtheit von 137 Frauen schwierig, aussagekräftige Ergebnisse insbesondere für die Altersgruppen an den Extremen der Altersverteilung (unter 30 und über 40 Jahre) zu berechnen. Mit demselben Problem war auch die amerikanische Studie von Doyle et al. (2016) konfrontiert, welche die theoretische Geburtenchance, gestützt auf 128 Zyklen zur Befruchtung von vitrifizierten Eizellen, berechnete. Weil insbesondere wenige Daten zu Frauen im Alter von 41 und 42 Jahren vorhanden waren, entschlossen sich die amerikanischen Autoren, ebenfalls 18'746 Zyklen mit frischen Eizellen in die Berechnung der theoretischen 
Geburtenchance einzuschliessen. Die Berechnung beruht darum im Wesentlichen auf den Daten von unfruchtbaren Paaren. Die Autoren sind der Ansicht, dass die theoretische Geburtenchance besser sein dürfte bei den fruchtbaren Frauen, die ihre Eizellen aus sozialen Gründen kryokonservieren lassen. Die zweite amerikanische Studie (Goldman et al. 2017) wollte eine möglichst gute Vergleichbarkeit mit diesen Frauen sicherstellen und hat darum nur die Daten von Frauen in die Berechnung eingeschlossen, bei denen zwar die Eileiter, nicht aber die Eizellen beeinträchtigt waren (43), oder bei denen nur der Partner unfruchtbar (423) war. Dies führt jedoch möglicherweise zu einem zu positiven Resultat. Wie die spanische (Cabo et al. 2016) und andere Studien (vgl. Kapitel 3.1.2) zeigen, leidet eine Minderheit der Frauen, die ihre Eizellen aus sozialen Gründen kryokonservieren lassen, unter einer eingeschränkten Fruchtbarkeit beispielsweise aufgrund von Endiometriose.

- Drittens wurde die theoretische Geburtenchance unterschiedlich berechnet. In der Studie von Doyle et al. (2016) wurde für die Modellierung von einer Binomialverteilung der Geburtenrate pro aufgetauter Oozyte für verschiedene Altersgruppen ausgegangen. Diese Berechnungsmethode stützt sich auf die Annahme, dass alle Eizellen dasselbe Potenzial haben, sich zu einem Kind zu entwickeln. Weil dies nicht realistisch ist, werden in der spanischen Studie Kaplan-Meier-Kurven für die Berechnung der theoretischen Geburtenchance verwendet. Bei dieser Berechnung ändert sich die Aussicht auf ein Kind, je nachdem wie viele Eizellen bereits aufgetaut und befruchtet wurden (Cabo et al. 2016). Ähnliche Überlegungen wurden auch bei der Berechnung der theoretischen Geburtenchance im FertiPROTEKTRegister angestellt (von Wolff et al. 2015). Das Modell zur Berechnung der theoretischen Geburtenchance in der zweiten amerikanischen Studie (Goldman et al. 2016) umfasst zwei Komponenten. Einerseits wurde mittels einer Poisson-Regression der Anteil reifer Eizellen berechnet, die sich je nach Alter der Frau zu einer Blastozyste weiterentwickeln. Andererseits wurde in das Modell die Wahrscheinlichkeit eingerechnet, dass eine Blastozyste euploid ist, das heisst keine Chromosomenanomalien aufweist. Je älter eine Frau ist, desto weniger euploide Blastozysten hat sie. Das Modell stützt sich auf die Annahme, dass jede euploide Blastozyste unabhängig vom Alter der Frau ein vergleichbares Entwicklungspotenzial hat. 
Zusammenfassend kann die theoretische Geburtenchance, gestützt auf die heterogenen Studien, nicht abschliessend beurteilt werden. Zudem lassen sich solche Daten nur bedingt auf jede einzelne Frau übertragen, weil grosse interindividuelle Unterschiede bezüglich Eizellreserve und Fruchtbarkeitschancen bestehen (NEK 2017). Gesichert scheint, dass die theoretische Geburtenchance stark vom Alter der Frau, der Anzahl entnommener Eizellen und der Erfahrung der Klinik abhängt (Nawroth 2015). Für eine realistische Chance auf eine Schwangerschaft wird Frauen zurzeit die Entnahme von mindestens 15 bis 20 Eizellen empfohlen (NEK 2017, Van der Ven 2017). Dafür sind in der Regel ein bis zwei Stimulationszyklen notwendig. Idealerweise erfolgt die Entnahme der Eizellen vor dem 35. Lebensjahr und bei einer guten Ovarreserve spätestens mit 38 Jahren (von Wolff et al. 2015).

\subsection{Anwendung der Technologie in der Schweiz}

Die Angaben in diesem Kapitel stammen aus einer Onlinebefragung bei Reproduktionsmedizinerinnen und -medizinern sowie explorativen Interviews bei vier Reproduktionsmedizinern. Ebenfalls einbezogen werden punktuell Aussagen von zwei interviewten Frauen, die Social Freezing bereits in Anspruch genommen haben und ihre Eizellen kryokonservieren liessen.

\subsubsection{Anbieter des Social Freezing}

Von den 30 Fertilitätskliniken in der Schweiz machten 17 keine Angaben zu ihrem Angebot in Bezug auf Social Freezing. Zwölf Kliniken gaben an, dass sie Social Freezing anbieten. Sieben sind in der Deutschschweiz, drei in der Westschweiz und zwei im Tessin angesiedelt. Gemäss einem interviewten Reproduktionsmediziner ist allerdings davon auszugehen, dass die meisten Kliniken Social Freezing im Angebot haben. Eine Klinik gab in der Onlinebefragung an, dass sie bisher aufgrund der mangelnden Nachfrage darauf verzichtet hat, Social Freezing anzubieten. Sie kann es sich allerdings vorstellen, dass diese Technologie in den nächsten Jahren Bestandteil ihres Angebots wird. 
Von neun der 30 online befragten Kliniken liegen zudem Angaben vor, wie sich ihre personellen Ressourcen auf die Behandlung von unfruchtbaren Paaren sowie die Kryokonservierung bei Krebspatientinnen und im Rahmen des Social Freezing verteilen. Die neun Kliniken wenden zwischen 71 und 100 Prozent ihrer personellen Ressourcen für die Behandlung von unfruchtbaren Paaren auf. Für die Kryokonservierung der Eizellen von Krebspatientinnen werden zwischen 2 und 12 Prozent der personellen Ressourcen eingesetzt. Universitätsund Kantonsspitäler setzen in diesem Bereich mehr Ressourcen ein als private Kliniken, was auch die interviewten Reproduktionsmediziner bestätigt haben. Für Social Freezing werden zwischen 2 und 15 Prozent der personellen Ressourcen aufgewendet. In fünf Kliniken sind es 3 Prozent oder weniger und in vier Kliniken zwischen 8 und 15 Prozent. Unterschiede zwischen Universitätsspitälern, Kantonsspitälern und privaten Kliniken sind anhand der Daten nicht erkennbar.

Diese neun Kliniken lieferten zudem Informationen zur Anzahl Frauen, denen sie pro Jahr mit den bestehenden Ressourcen eine Eizellentnahme anbieten könnten. Die Spannbreite bewegt sich zwischen 20 und 1000 Frauen pro Jahr. Fünf Kliniken könnten 100 Frauen und weniger betreuen. Bei zwei Kliniken sind es zwischen 400 und 500 Frauen. Weitere zwei Kliniken könnten 1000 Frauen behandeln. Alle neun Kliniken zusammen könnten 3200 Frauen die Kryokonservierung von Eizellen anbieten. Zwei interviewte Reproduktionsmediziner gehen davon aus, dass sie die Anzahl Eizellentnahmen pro Tag und somit die Anzahl behandelter Frauen pro Jahr verdoppeln könnten. Ein interviewter Reproduktionsmediziner geht davon aus, dass seine Klinik die Anzahl Behandlungszyklen um 50 Prozent steigern könnte. Gestützt auf diese Aussagen der drei Reproduktionsmediziner sowie die aktuellen Daten des Bundesamtes für Statistik zur Fortpflanzungsmedizin könnte neben den bisher jährlich 6000 behandelten Frauen weiteren 3000 bis 6000 Frauen eine Kryokonservierung der Eizellen angeboten werden.

Allerdings wurde von einem Reproduktionsmediziner in der Onlinebefragung angemerkt, dass sich die Kapazitäten der Kliniken kontinuierlich verändern und darum auch die Kapazitäten für Social Freezing schwer voraussehbar sind. Gemäss einem interviewten Reproduktionsmediziner müssen die Eizellen nach der Entnahme möglichst schnell kryokonserviert werden, um ein optimales Behandlungsresultat zu erzielen und mit einer hohen Wahrscheinlichkeit die Geburt eines Babys zu gewährleisten. Gleichzeitig werden aber auch täglich Eizel- 
len von unfruchtbaren Paaren entnommen, die nach der Entnahme in vitro befruchtet werden müssen. Ohne die klinikinternen Abläufe zum Beispiel in Zusammenhang mit einem Eizellspendeprogramm zu ändern, könnten die Kapazitäten für Social Freezing nicht massiv ausgeweitet werden.

Die in den Kliniken vorhandenen Kapazitäten für Social Freezing übersteigen somit die aktuelle Nachfrage (vgl. Kapitel 3.2.1). Im Gegensatz zu den USA und Grossbritannien, wo Fertilitätskliniken beispielsweise Egg-Freezing-Partys veranstalten, wird in der Schweiz zurzeit nicht offensiv für Social Freezing geworben. Das würde gemäss zwei interviewten Reproduktionsmedizinern in der Öffentlichkeit negativ aufgenommen. Viele Kliniken informieren auf ihrer Homepage über Social Freezing. Daneben würde indirekt über die Präsenz in den Medien und bei Kongressen von Gynäkologen für die Technologie geworben. In einer Klinik wurde zudem eine Broschüre entwickelt, die in den Praxen der Gynäkologen aufgelegt werden soll. Die Gynäkologen würden die Möglichkeiten, die Social Freezing bietet, allerdings noch nicht sehr gut kennen. Zwar würden einzelne Frauen von ihrem Gynäkologen überwiesen, aber in der Regel würden sich die Frauen aufgrund von Informationen in den Medien oder von Bekannten in der Klinik melden. Eine der befragten Frauen wurde in Zusammenhang mit den Diskussionen in den Medien zum Entscheid von Apple und Facebook, Social Freezing zu finanzieren, auf die Technologie aufmerksam. Bei einer anderen war es eine Freundin, die ihre Eizellen ebenfalls einfrieren lies.

\subsubsection{Information der Frauen}

Aus der Abbildung 4 wird ersichtlich, welche Inhalte im Rahmen der Beratung der Frauen angesprochen werden und welche zusätzlich vertraglich geregelt sind. Acht der 30 online befragten Kliniken beantworteten die entsprechenden Fragen. Die Kosten werden in allen dieser acht Kliniken thematisiert und vertraglich geregelt. Wenn sich die Frauen bei den Kliniken telefonisch nach Social Freezing erkundigen, sind die Kosten nach Auskunft der interviewten Reproduktionsmediziner ein wichtiges Thema. Bevor sich die Frauen ausführlich beraten liessen, wüssten sie in der Regel über die Kosten Bescheid. Diese variieren zwischen den Kliniken. Die Kosten für die ovarielle Stimulation und die Eizellentnahme bewegen sich in drei Kliniken zwischen 4600 und 5300 Franken. Mit den Kosten für die Medikamente, die je nach Frau höher oder tiefer ausfallen, 
müssen die Frauen rund 6000 Franken für die Kryokonservierung der Eizellen bezahlen. Dazu kommen die jährlichen Lagerungskosten von rund 200 Franken.

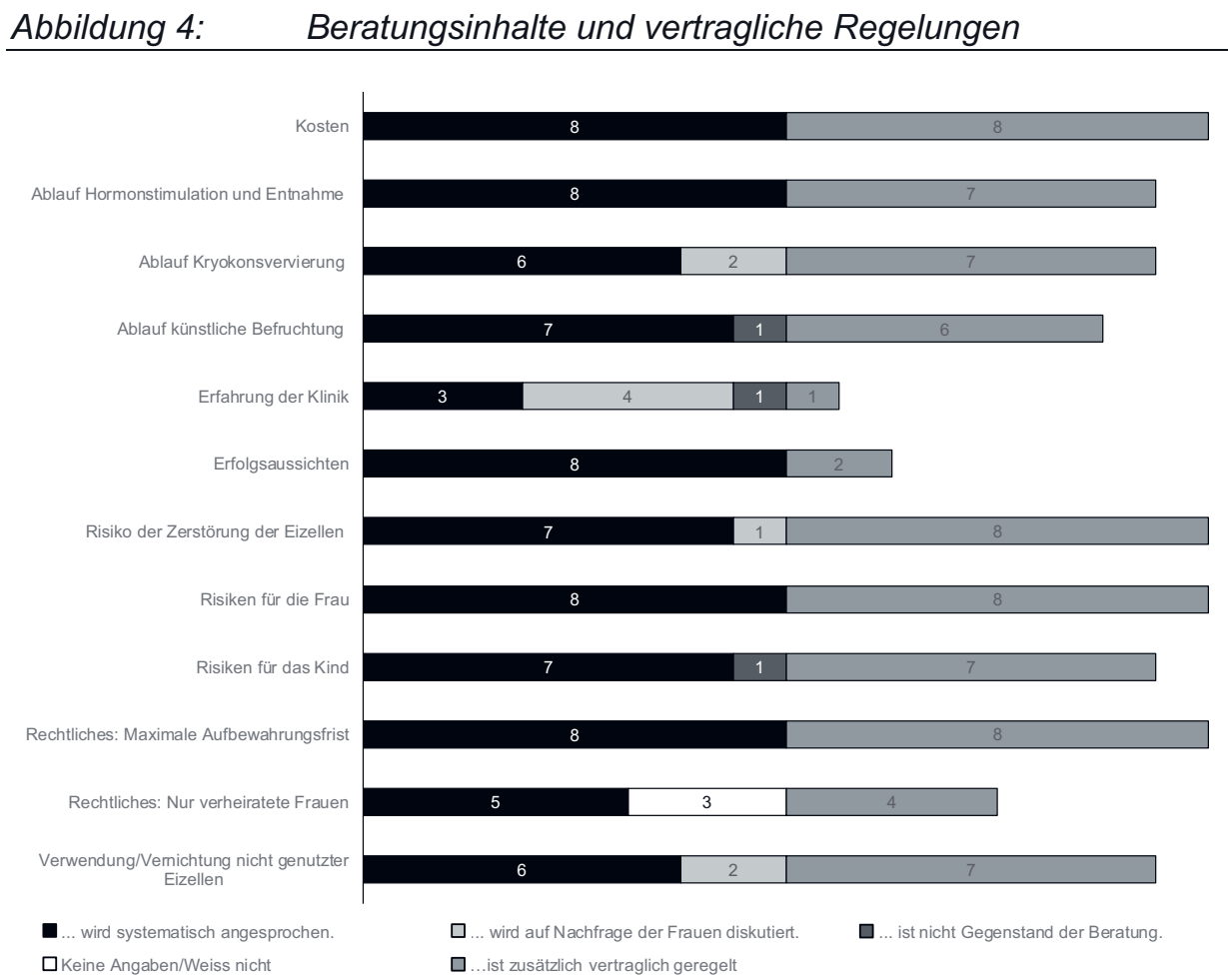

Quelle: Befragung der Reproduktionsmediziner ( $n=8$ für alle Fragen ausser «Rechtliches: maximale Aufbewahrungsfrist, wo zwei Reproduktionsmediziner keine Angaben lieferten).

In allen acht Kliniken wird das Verfahren zur ovariellen Stimulation und zur Eizellentnahme systematisch angesprochen und in sieben ist es zusätzlich vertraglich geregelt. Auch die weiteren Etappen - die Kryokonservierung und die Befruchtung der Eizellen in vitro - werden in den meisten Kliniken besprochen und vertraglich geregelt.

Nur in drei Kliniken werden die Frauen systematisch über die Erfahrungen der Klinik informiert und lediglich in einer Klinik ist dieser Punkt Bestandteil eines 
Vertrags. Ein interviewter Reproduktionsmediziner erachtet es als wichtig, dass die Frauen über die Erfahrungen der Klinik informiert werden und sie sich eine Zweitmeinung einholen. Die Erfolgsaussichten, das heisst die Baby-TakeHome-Rate wird hingegen systematisch angesprochen, aber nur in zwei Kliniken vertraglich geregelt. Die Angaben zur Baby-Take-Home-Rate beruhen auf unterschiedlichen Quellen. Gemäss einem interviewten Reproduktionsmediziner orientiert sich die Klinik an den Daten aus anderen ausländischen Kliniken. In einer zweiten Klinik dienen die eigenen Daten zur Baby-Take-Home-Rate bei unfruchtbaren Paaren als Orientierung. In einer dritten Klinik werden regelmässig Eizellen von unfruchtbaren Paaren eingefroren, weil nach der Eizellentnahme keine Spermien des Mannes vorliegen. Die Baby-Take-Home-Rate basiert auf den klinischen Daten zur Entwicklung dieser Eizellen. In einer vierten Klinik basiert die Baby-Take-Home-Rate bei Frauen im Alter von 42 und 43 Jahren auf Daten aus grossen Registern und bei den anderen Altersgruppen auf eigenen klinischen Daten. Je nach Patientengeschichte und Alter der Frau wird die Baby-Take-Home-Rate angepasst. Zudem sei es wichtig, darauf zu verweisen, dass Social Freezing keine Garantie für ein Baby sei.

Auch die Risiken für die Eizellen, die Frau und das Kind werden in den acht Kliniken mehrheitlich systematisch angesprochen und vertraglich geregelt. In einer Klinik werden die Risiken für das Kind nicht angesprochen. Gemäss einem interviewten Reproduktionsmediziner seien Pannen bei der Kryokonservierung der Eizellen sehr selten und die meisten Kliniken hätten für solche Fälle eine Haftpflichtversicherung. Die interviewten Reproduktionsmediziner erachten die Risiken für Mutter und Kind als gering. Weltweit würden mehrere Millionen in vitro gezeugter Kinder aufwachsen. Bei diesen würden häufiger sehr seltene Krankheiten auftreten und die Fehlbildungsrate sei leicht erhöht. Auf die epigenetischen Risiken wird in der Regel nicht eingegangen, weil die Evidenz noch gering sei und die Frauen nicht verunsichert werden sollen. Die untersuchten Studienpopulationen seien zu klein für aussagekräftige Resultate. Zudem hätten sich die reproduktionsmedizinischen Verfahren weiterentwickelt und es sei darum unklar, ob die heute in vitro gezeugten Kinder den gleichen Risiken ausgesetzt seien wie die vor zehn bis 20 Jahren in vitro gezeugten Kinder, deren Gesundheit zurzeit untersucht wird. Schliesslich sei es schwierig, die epigenetischen Risiken verständlich zu erläutern. Ein Reproduktionsmediziner gibt an, dass er auf Nachfrage die Risiken für das Kind ausführlich erläutert, aber das Thema nicht systematisch ansprochen wird. Eine der interviewten Frauen hatte im Internet nach wissenschaftlichen Studien gesucht und unterhielt sich mit 
ihrem Arzt über die langfristigen gesundheitlichen Folgen für das Kind. Die zweite Frau holte sich vor allem über Internetforen persönliche Erfahrungen von anderen Frauen mit Social Freezing ein. Über Risiken für die Frau und das Kind informierte sie sich bewusst nicht ausführlich, um sich keine allzu grossen Sorgen machen zu müssen. Die Risiken für das Kind waren auch kaum Gegenstand ihrer Beratung.

Was die rechtlichen Rahmenbedingungen anbelangt, ist die maximale Aufbewahrungsfrist von zehn Jahren in allen acht online befragten Kliniken Bestandteil der Beratung und der Verträge mit den Frauen. Die Verwendung respektive Vernichtung nicht genutzter Eizellen wird ebenfalls in den meisten Kliniken angesprochen und vertraglich geregelt. Die beiden interviewten Frauen haben sich allerdings noch nie mit dem Thema auseinandergesetzt und hätten es begrüsst, von der Klinik darauf angesprochen zu werden. Hingegen informieren nur fünf Kliniken die Frauen darüber, dass sie ihre Eizellen nur mit den Spermien ihres Partners befruchten lassen dürfen und nicht auf Spenderspermien zurückgreifen können. Dieser Punkt ist auch nur in der Hälfte der befragten Kliniken vertraglich geregelt.

\subsubsection{Behandlung der Frauen}

Nach Auskunft der interviewten Reproduktionsmediziner lassen sich die allermeisten Frauen nach der Beratung ihre Eizellen entnehmen und kryokonservieren. Nur in einzelnen Fällen verzichten die Frauen auf die Behandlung, weil sich beispielsweise die Erfolgsaussichten aufgrund des fortgeschrittenen Alters als zu gering erwiesen.

Die interviewten Frauen erlebten die Behandlung unterschiedlich. Eine machte sehr gute Erfahrungen mit dem Ärzte- und Pflegeteam. Die Behandlung mittels Spritzen sowie die Medikamente vertrug sie gut. Allerdings hatte sie nach der Eizellentnahme während zweier Wochen einen angeschwollenen Bauch. Sie entschied sich für eine zweite Stimulationsrunde. Die Erfahrungen der zweiten Frau waren deutlich schlechter. Nach der Eizellentnahme fiel ihre Menstruation über mehrere Monate aus. Sie fühlte sich körperlich und in der Folge auch psychisch stark belastet. Sowohl die Vorbereitung auf diese Nebenwirkungen der Behandlung als auch die Nachbetreuung nach dem Eingriff erlebte sie als unbe- 
friedigend. Sie verzichtete auf einen zweiten Stimulationszyklus, weil sie eine ausreichend hohe Anzahl Eizellen kryokonservieren lassen konnte. Beide Frauen bedauern den Eingriff jedoch nicht und würden Social Freezing anderen Frauen empfehlen.

Das Maximalalter für die Eizellentnahme variiert je nach Klinik (vgl. Abbildung 5). Während einzelne Kliniken die Alterslimite bei Mitte 30 und somit relativ früh ansetzen, ist Social Freezing in anderen Kliniken auch noch mit über 40 möglich. Nach Auskunft eines interviewten Reproduktionsmediziners wird Frauen über 38 zwar vom Social Freezing abgeraten, aber die Technologie auch nicht verweigert.

Abbildung 5: $\quad$ Alterslimite für die Eizellentnahme

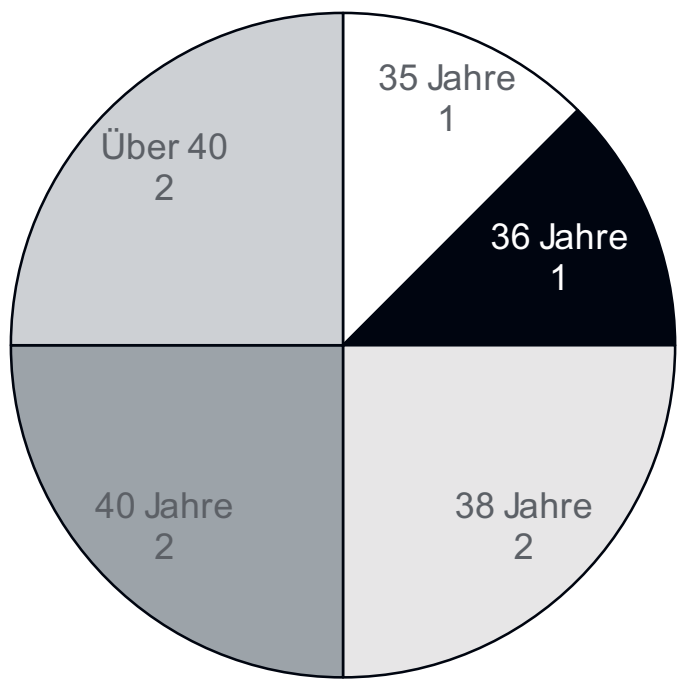

Quelle: Befragung der Reproduktionsmediziner $(n=8)$.

Im Rahmen der Onlinebefragung wurden die Kliniken gefragt, wie viele Eizellen durchschnittlich in verschiedenen Altersgruppen eingefroren werden. Die Antworten fielen sehr heterogen aus. Es ist daher nicht klar, ob die Antworten verschiedene Praktiken spiegeln oder ob die Frage unterschiedlich interpretiert 
wurde. Gemäss den interviewten Reproduktionsmedizinern sollten bei 35-Jährigen zwischen zehn und 20 Eizellen und bei 35- bis 38-Jährigen 20 Eizellen entnommen werden. Dafür sind in der Regel zwei Stimulationszyklen und bei über 38-Jährigen drei bis vier Zyklen notwendig. Ein anderer Reproduktionsmediziner empfiehlt die Entnahme von 20 bis 30 Eizellen, um auch gegen eine allfällige Unfruchtbarkeit des künftigen Partners der Frau gewappnet zu sein. Die genaue Anzahl Stimulationszyklen ist abhängig von der Zahlungsbereitschaft sowie der Reaktion der Frau auf die ovarielle Stimulation. Gemäss einem Reproduktionsmediziner belassen es rund zwei Drittel bei einem Stimulationszyklus und rund zehn Eizellen.

\subsubsection{Gesetzliche Rahmenbedingungen}

In der Abbildung 6 sind die Ansichten der online befragten Reproduktionsmedizinerinnen und -mediziner zu den gesetzlichen Rahmenbedingungen dargestellt. Dazu liegen Angaben von neun der 30 befragten Kliniken vor. Diese zeigen, dass sich eine Mehrheit der Befragten eine Unterstellung der Kryokonservierung von Eizellen unter das Fortpflanzungsmedizingesetz vorstellen kann.

Eine Verlängerung respektive eine Aufhebung der Aufbewahrungsfrist von zehn Jahren für die kryokonservierten Eizellen stellt das wichtigste Anliegen der befragten Reproduktionsmedizinerinnen und -mediziner dar. Da zurzeit die meisten Frauen bei der Kryokonservierung ihrer Eizellen bereits 35 Jahre alt sind und in der Regel vor ihrem 45. Geburtstag auf ihre Eizellen zurückgreifen möchten, reicht nach Ansicht der interviewten Reproduktionsmediziner in der Regel eine Lagerungsfrist von zehn Jahren. Da die Qualität der Eizellen mit zunehmendem Alter abnimmnt, seien allerdings eine Eizellentnahme weit vor dem 35 . Geburtstag und eine längere Aufbewahrungsfrist sinnvoll. Als umständlich wird in der Praxis auch die Pflicht empfunden, nach fünf Jahren eine Verlängerung der Frist auf zehn Jahre zu beantragen. Die beiden befragten Frauen empfinden die Lagerfrist als diskriminierend. Sie sind der Ansicht, dass es ihre Eizellen sind und nur sie über diese verfügen dürfen. Mit dieser Lagerfrist kam Social Freezing in der Schweiz für sie nur infrage, weil die Möglichkeit besteht, die Eizellen ins Ausland zu transferieren. 


\section{Abbildung 6: $\quad$ Änderung der gesetzlichen Rahmenbedingungen}

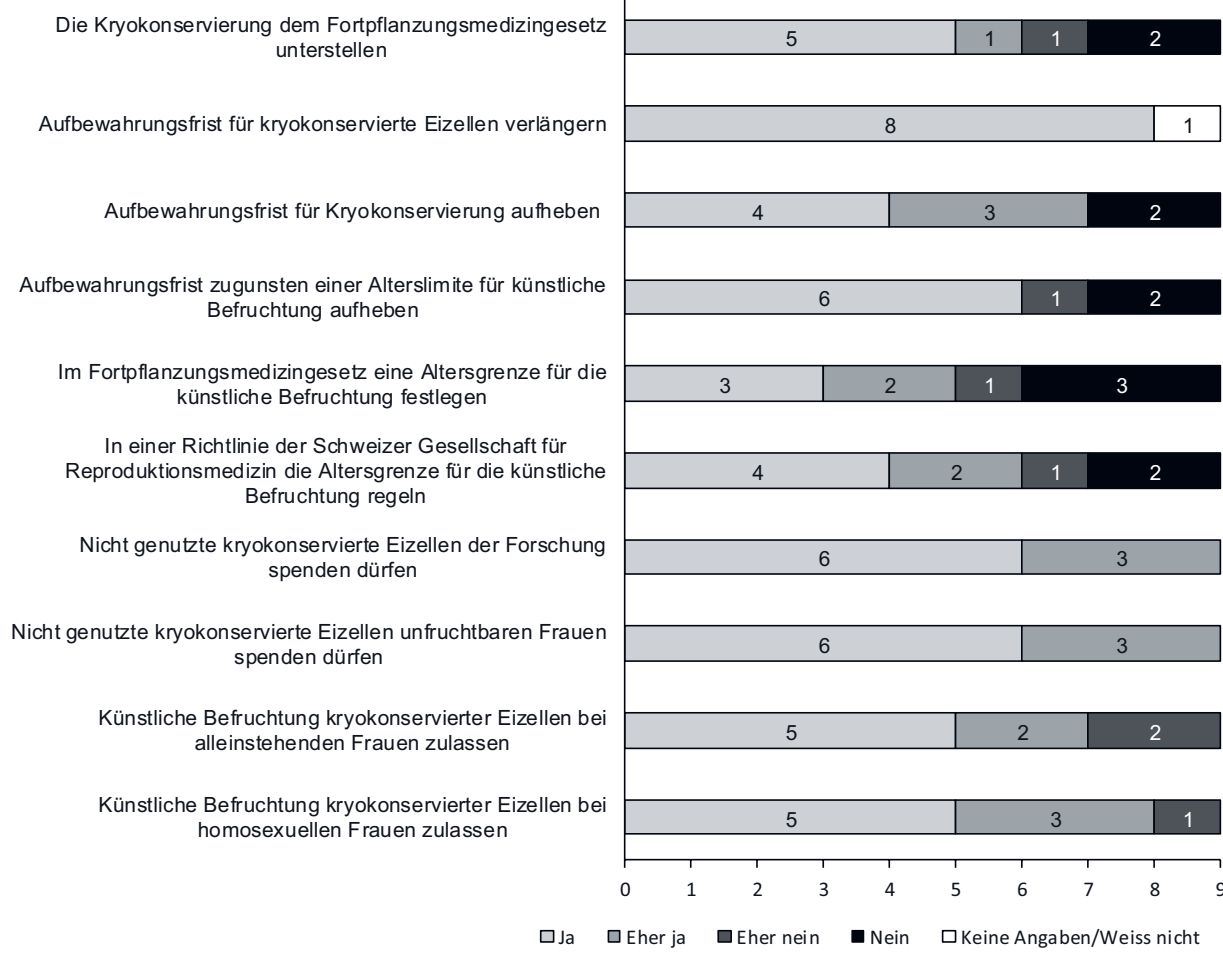

Quelle: Befragung der Reproduktionsmediziner $(n=9)$.

Die neun online befragten Reproduktionsmedizinerinnen und -mediziner können sich mehrheitlich eine Aufhebung der Aufbewahrungsfrist zugunsten einer Altersgrenze vorstellen. Allerdings äussern sich leicht mehr Befragte zugunsten einer Altersgrenze in den Richtlinien der Schweizerischen Gesellschaft für Reproduktionsmedizin als für eine gesetzliche Altersgrenze. Für eine gesetzliche Lösung sprechen sich fünf Reproduktionsmedizinerinnen und -mediziner aus, während vier eher dagegen sind. Aktuell bewegt sich das maximale Alter für eine In-vitro-Befruchtung in acht Kliniken zwischen 43 und 48 Jahren. Fünf Kliniken kennen ein Maximalalter von 45 Jahren. Ein interviewter Reproduktionsmediziner berichtet, dass zuerst ein gynäkologisches Gutachten vorliegen muss, bevor eine Befruchtung in vitro von Eizellen einer Frau in fortgeschritte- 
nem Alter vorgenommen wird. Bei einer engmaschigen gynäkologischen Überwachung seien Schwangerschaften bei Frauen Mitte 40 problemlos möglich, findet ein anderer Interviewter.

Alle befragten Reproduktionsmedizinerinnen und -mediziner sind zudem dafür oder eher dafür, dass überzählige Eizellen entweder der Forschung oder unfruchtbaren Frauen zur Verfügung gestellt werden. Ebenfalls eine Mehrheit würde die Befruchtung von kryokonservierten Eizellen bei alleinstehenden und homosexuellen Frauen zulassen. Auch eine der befragten Frauen würde es begrüssen, wenn alleinstehende Frauen ihre Eizellen in vitro befruchten lassen könnten. 


\section{Aktuelle Nachfrage nach Social Freezing}

Noch ist Social Freezing eine Nischentechnologie, das Interesse daran scheint jedoch stetig zu wachsen. Im folgenden Kapitel werden die Literaturbefunde zur Nachfrage nach Social Freezing im Ausland und in der Schweiz präsentiert.

\subsection{Internationale Studien zur Nachfrage in anderen Ländern}

In diesem Kapitel wird auf die Nachfrage nach Social Freezing in anderen europäischen Ländern eingegangen.

\subsubsection{Anzahl Frauen}

Die Datenlage bezüglich der Nachfrage nach Social Freezing ist sowohl im Ausland als auch in der Schweiz limitiert. Eine Arbeitsgruppe der europäischen Fachgesellschaft der Reproduktionsmedizinerinnen und -mediziner (ESHRE) publizierte im Jahr 2017 eine Übersichtsarbeit bezüglich Eizellkonservierung in 17 europäischen Ländern (vgl. Tabelle 3). Die Datenqualität variierte jedoch stark von Land zu Land. Relativ aussagekräftig waren die Daten zur Anzahl durchgeführter Zyklen zur Kryokonservierung von Eizellen. Im Jahr 2013 wurden in 17 europäischen Ländern insgesamt 9126 Zyklen durchgeführt, wovon 5620 Zyklen auf Spanien fielen (62 \%). Andere Länder folgen mit grossem Abstand (Grossbritannien 810, Frankreich 798, Italien 477, Tschechien 471, Belgien 386, Deutschland 235). In der Schweiz wurden im Jahr 201348 Zyklen zur Entnahme von Eizellen durchgeführt. 
Für 8885 Zyklen lagen Informationen zur Indikationsstellung vor (97,4 \%). 60 Prozent der Zyklen wurden zwecks Eizellspende, 27 Prozent aufgrund medizinischer Indikationen und 13 Prozent im Rahmen des Social Freezing durchgeführt. Von den 1165 Zyklen zwecks Social Freezing entfielen 497 auf Spanien (43\%), 366 auf Belgien (31\%), 257 auf Grossbritannien (22\%) und 29 auf Italien (3\%). Von den 17 untersuchten Ländern hatten sechs keine Daten zum Social Freezing erhoben, vier meldeten keine und drei nur einzelne Zyklen. Dazu zählt die Schweiz, in der im Jahr 2013 sieben Zyklen aus nicht medizinischen Gründen durchgeführt wurden. Die Anzahl durchgeführter Zyklen lieferte zwar Hinweise auf die Nachfrage nach Social Freezing. Da insbesondere in fortgeschrittenem Alter mehrere Zyklen notwendig sind, um eine ausreichende Fertilitätsreserve zu bilden, kann die Anzahl Zyklen aber nicht mit der effektiven Nachfrage gleichgesetzt werden.

Tabelle 3:

Übersicht über die Kryokonservierung von Eizellen in 17 europäischen Ländern

\begin{tabular}{|c|c|c|c|c|c|c|c|}
\hline \multirow[t]{2}{*}{ Countries } & \multicolumn{3}{|c|}{ Total numbers } & \multicolumn{4}{|c|}{ Numbers per OcC indications } \\
\hline & $\begin{array}{l}\text { Total ART } \\
\text { aspirations }\end{array}$ & $\begin{array}{l}\text { OoC } \\
\text { aspirations }\end{array}$ & $\begin{array}{l}\text { Oocytes } \\
\text { number }\end{array}$ & $\begin{array}{l}\text { OD } \\
\text { cycles/ } \\
\text { oocytes }\end{array}$ & $\begin{array}{l}\text { Serious } \\
\text { disease } \\
\text { cycles/ } \\
\text { oocytes }\end{array}$ & $\begin{array}{l}\text { Other } \\
\text { medical } \\
\text { cycles/ } \\
\text { oocytes }\end{array}$ & $\begin{array}{l}\text { Non- } \\
\text { medical } \\
\text { cycles/ } \\
\text { oocytes }\end{array}$ \\
\hline Belarus & $2 ’ 000$ & 3 & 7 & $0 / 0$ & $3 / 7$ & $3 / 7$ & $0 / 0$ \\
\hline Belgium & $199^{\prime} 590$ & 386 & $3{ }^{\prime} 750$ & NA & NA & NA & $366 / 2 ' 698$ \\
\hline Czech Rep & $18 ' 574$ & 471 & 5799 & NA & $302 / 451$ & $302 / 451$ & NA \\
\hline Estonia & 1'836 & 4 & 31 & $0 / 0$ & $2 / 23$ & $2 / 23$ & $2 / 8$ \\
\hline Finland & $4{ }^{\prime} 861$ & 23 & NA & NA & NA & NA & NA \\
\hline France & $66^{\prime} 2235$ & 798 & NA & $324 / \mathrm{NA}$ & 474/NA & 474/NA & $0 / 0$ \\
\hline Germany & $566^{\prime} 075$ & 235 & 1'350 & NA & NA & NA & NA \\
\hline Greece & NA & 34 & 203 & $10 / 35$ & $8 / 105$ & $8 / 105$ & $7 / 33$ \\
\hline Hungary* & 3'535 & 5 & 36 & NA & $4 / 27$ & $4 / 27$ & NA \\
\hline Italy & $50 ' 174$ & 477 & 3'689 & $152 / 1^{\prime} 456$ & $296 / 199$ & 296/1'999 & $29 / 234$ \\
\hline Malta & 100 & 41 & NA & $0 / 0$ & $41 / \mathrm{NA}$ & $41 / \mathrm{NA}$ & NA \\
\hline Romania & 2'156 & NA & NA & NA & NA & NA & NA \\
\hline Slovenia & $3 \prime 668$ & 16 & 210 & $9 / 135$ & $7 / 75$ & $7 / 75$ & $0 / 0$ \\
\hline Spain & $54^{\prime} 129$ & 5620 & NA & $262 / 1^{\prime} 970$ & $8 / 60$ & $8 / 60$ & $497 / 3^{\prime} 738$ \\
\hline Switzerland & 4'964 & 48 & NA & $41 / \mathrm{NA}$ & NA & NA & 7/NA \\
\hline UK & 4'6421 & 810 & 7’042 & 165/1'366 & $270 / 2 ' 462$ & $270 / 2462$ & 257/2'115 \\
\hline Ukraine & $12^{\prime} 707$ & 155 & 1'538 & $3 / 21$ & $16 / 163$ & $16 / 163$ & $0 / 0$ \\
\hline Total: 17 & 343 '025 & 9'126 & $23 ’ 655$ & 966/4'983 & $1431 / 5 ’ 372$ & 1'431/5'372 & 1'165/8'826 \\
\hline
\end{tabular}

Quelle: ESHRE Working Group on Oocyte Cryopreservation in Europe, F. Shenfield, J. de Mouzon, G. Scaravelli, M. Kupka, A.P. Ferraretti, F.J. Prados, V. Goossens; Oocyte and ovarian tissue cryopreservation in European countries: statutory background, practice, storage and use, Human Reproduction Open, Volume 2017, Issue 1, 29 March 2017. 


\subsubsection{Soziodemografische Merkmale der Frauen}

Zum demografischen Profil und zur Motivation von Frauen, Social Freezing in Anspruch zu nehmen, liegen drei Typen von Studien vor. Der erste Typ umfasst quantitative Befragungen von Patientinnen reproduktionsmedizinischer Zentren, die Social Freezing in Anspruch genommen haben in Kalifornien (Meyers et al. 2015), in New York (Hodes-Wetz et al. 2013) in Australien (Hammarberg et al. 2017, Pritchard et al. 2017) und in Belgien (Stoop et al. 2015). In diesen vier Studien wurden zwischen 49 (Meyers et al. 2015) und 183 (Hodes-Wetz et al. 2013) Frauen befragt. Während der Rücklauf in den amerikanischen Befragungen relativ tief war, betrug der Rücklauf in der australischen Befragung 53 Prozent und in der belgischen Untersuchung 70 Prozent. Der hohe Rücklauf in der belgischen Studie dürfte darauf zurückzuführen sein, dass die Befragung telefonisch erfolgte, während die Frauen in den anderen drei Studien schriftlich befragt wurden.

Der zweite Typ von Studien betrifft die Auswertung von Datensätzen. In der Studie von Vallejo et al. 2013 wurden verschiedene Daten aus mehreren amerikanischen reproduktionsmedizinischen Zentren aufbereitet und ausgewertet. Dazu zählen Informationen aus psychologischen Beratungen von Patientinnen, eine Befragung des Gesundheitspersonals in den Zentren sowie klinische Daten. Die einzigen Daten zum demografischen Profil der Frauen aus dem deutschsprachigen Raum stammen aus dem Register des FertiPROTEKT, in dem reproduktionsmedizinische Zentren aus Deutschland, Österreich und der Schweiz mitarbeiten. In der Schweiz zählen dazu die Abteilung Gynäkologische Endokrinologie und Reproduktionsmedizin des Inselspitals Bern sowie das Institut Fiore in St. Gallen. Im FertiPROTEKT-Register wurden die demografischen Daten von 134 Frauen erfasst und ausgewertet (von Wolff et al. 2015). Ebenso liefert eine spanische Studie von 1468 Frauen Informationen zu den soziodemografischen Merkmalen und zur Verwendung der Eizellen (Cabo et al. 2016).

Der dritte Typ umfasst sozialwissenschaftliche Studien, basierend auf qualitativen Interviews. Kylie Louise Baldwin hat in einer umfassenden Dissertation (2016) aufgezeigt, dass die Gründe für die Nutzung des Social Freezing mehrschichtig sind und sowohl von makro- als auch von mikrosoziologischen Faktoren beeinflusst werden. Grundlage für ihre Dissertation bildeten eine Medienanalyse sowie Interviews mit 23 englischen Frauen, die ihre Eizellen aus sozialen Gründen kryokonservieren liessen. Cathrine Waldby (2015) interviewte 
15 Frauen sowie zehn Mitarbeitende zweier reproduktionsmedizinischer Kliniken in London. Daneben wurden in den USA im Rahmen einer ethnografischen Dissertation Frauen qualitativ zum Social Freezing befragt (Romain 2011).

Obwohl diese Studien methodisch recht unterschiedlich und in verschiedenen Ländern durchgeführt worden sind, zeichnen sie ein ähnliches Bild der soziodemografischen Merkmale wie Alter, Partnerschaft und Ausbildung der Frauen, die ihre Eizellen aus sozialen Gründen kryokonservieren lassen.

- Über 35-jährig: In den beiden amerikanischen Befragungen waren 84 Prozent (Hodes-Wertz et al. 2013) respektive 90 Prozent der Frauen (Myers et al. 2015) über 35 Jahre alt. In der australischen Befragung lag das Durchschnittsalter der Frauen bei 37,1 Jahren (Hammarberg et al. 2017) und bei den befragten belgischen Frauen bei 36,9 Jahren (Stoop et al. 2015). Im deutschsprachigen Raum waren 51 Prozent der Frauen zwischen 35 und 39 Jahre und 23 Prozent über 40 Jahre (von Wolff et al. 2015). In der spanischen Studie waren die Frauen im Schnitt 37,2 Jahre alt und 79,2 Prozent waren über 37 Jahre alt (Cabo et al. 2016). Auch die amerikanischen und englischen Frauen, die qualitativ befragt wurden, waren mehrheitlich über 35 Jahre alt (Baldwin et al. 2015, Waldby 2015, Romain 2011). Social Freezing wird jedoch idealerweise vor dem 35. Altersjahr durchgeführt, weil danach die Eizellqualität und somit die Erfolgsaussichten dieser Technologie progressiv abnehmen (vgl. Kapitel 2.3.2).

- Mehrheitlich Singles: 90 Prozent der befragten australischen Frauen (Pritchard et al. 2017), 84 Prozent der amerikanischen Frauen (Hodes-Wertz et al. 2013), 80 Prozent der deutschsprachigen Frauen im FertiPROTEKTRegister und 75,6 Prozent der spanischen Frauen (Cabo et al. 2016) waren bei der Einfrierung der Eizellen alleinstehend. Auch die qualitativ befragten englischen Frauen waren mehrheitlich Singles (Baldwin et al. 2015, Waldby 2015).

- Gut ausgebildet: Sowohl in den qualitativen Befragungen (Baldwin et al. 2015, Waldby 2015, Romain 2011) als auch in zwei quantitativen Studien verfügten die Frauen mehrheitlich über eine tertiäre Ausbildung. Bei den befragten australischen Frauen waren es 90 Prozent (Pritchard et al. 2017), bei den deutschsprachigen Frauen 76 Prozent (von Wolff et al. 2015) und bei den spanischen Frauen 72,8 Prozent (Cabo et al. 2016). 
- Teilweise unfruchtbar: Einzelne Studien berichten, dass die Frauen nicht nur aus sozialen Gründen, sondern teilweise aufgrund einer effektiven oder befürchteten medizinischen Diagnose wie polyzystisches Ovariensyndrom, Endiometriose, verklebte Eileiter, verfrühte Menopause und Krebs in der Familie ihre Eizellen einfrieren lassen. In der Befragung von Baldwin (2015) betraf dies fünf von 23 befragten englischen Frauen. Von den befragten 183 amerikanischen Frauen berichteten 9 Prozent, dass bei innen bereits vor Inanspruchnahme des Social Freezing eine reduzierte Fruchtbarkeit diagnostiziert worden war (Hodes-Wetz et al. 2013). In einer spanischen Studie froren 6 Prozent der Frauen ihre Eizellen aus medizinischen und sozialen Gründen ein (Cabo et al. 2016).

\subsubsection{Motivation der Frauen}

In den im vorhergehenden Kapitel erwähnten Studien wurde auch die Motivation der Frauen erfasst, ihre Eizellen einfrieren zu lassen. Die umfassendsten Informationen bieten die qualitativen soziologischen (Baldwin 2016, Waldby 2015) respektive ethnografischen Studien (Romain 2011). Deren Befunde decken sich auf weiten Strecken mit den Beobachtungen aus Studien über mögliche Gründe, die zu einem Hinauszögern der Mutterschaft generell (Daniluk und Koert 2017, Martin 2017, Perrier 2013, Budds et al. 2016) sowie bei Paaren zu einer Fertilitätsbehandlung (Vialle et al. 2016, Woodward und MacDuffie 2016) führen. Nachfolgend werden die wichtigsten Motivationen der Frauen erläutert.

\section{Social Freezing als Instrument zur Karriereplanung}

In den Medien wird den Frauen, die ihre Eizellen kryokonservieren lassen, unterstellt, sie wählten diese Technologie primär, um ihre Karriere voranzutreiben und die Mutterschaft bewusst auf einen späteren Zeitpunkt zu verschieben (Baldwin 2017, Mertes 2013). Diese Unterstellung wird aber von den von Baldwin befragten (2017) englischen Frauen zurückgewiesen, die ihre Eizellen kryokonservieren liessen. Ihr Berufsleben respektive ihre Berufskarriere hatte keinen Einfluss auf ihre Familienplanung oder die Inanspruchnahme des Social Freezing. Zudem geben die englischen Frauen an, dass sie die Familiengründung nicht bewusst hinausgeschoben haben. Stattdessen führen sie ihre Situa- 
tion auf verschiedene Umstände ausserhalb der individuellen Kontrolle zurück. Dieser Befund spiegelt sich auch in den Beobachtungen in der Literatur über verspätete Mutterschaft (Daniluk und Koert 2017, Perrier 2017, Budds et al. 2016) respektive zur Nutzung von reproduktionsmedizinischen Verfahren (Vialle et al. 2016, Martin 2017).

\section{Social Freezing für mehr Spielraum bei der Partnersuche}

Der mit Abstand am meisten zitierte Grund für die Inanspruchnahme des Social Freezing betrifft das Fehlen eines geeigneten Partners (Stoop et al. 2015, Pritchard et al. 2017, Hodes-Wertz et al. 2013, Myers et al. 2015, Waldby 2015, Baldwin 2016). Dieser Grund wird auch in Studien zu verspäteter Mutterschaft generell respektive zu später Elternschaft bei unfruchtbaren Paaren als wichtig erachtet (Daniluk und Koert 2017, Perrier 2013, Vialle et al. 2016). Social Freezing wird von den Frauen als Möglichkeit erachtet, den Druck aus der Partnersuche zu nehmen (Stoop et al. 2015, Vallejo et al. 2013, Waldby 2015) und sich zwecks Erfüllung des Kinderwunsches nicht in eine missliebige Beziehung zu stürzen (Baldwin 2016). Gemäss Baldwin wünschen sich die von ihr befragten englischen Frauen, dass sich der ideale Vater gemeinsam mit der Mutter für die Kindererziehung verantwortlich fühlt und sich stark darin engagiert. Diese «neue Vaterfigur» genoss bei den von Baldwin befragten Frauen eine hohe Wertschätzung.

\section{Social Freezing zur Aufrechterhaltung genetischer Familienbeziehungen}

Für viele Frauen, die ihre Eizellen einfrieren lassen, ist eine genetische Verbindung zwischen Mutter und Kind von Bedeutung (Baldwin 2016, Romain 2011, Waldby 2015). So sprachen sich 96 Prozent der 183 befragten amerikanischen Frauen für eine natürliche Zeugung aus, 93 Prozent könnten sich eine reproduktionsmedizinische Zeugung mittels eigener Eizellen vorstellen. Eine Eizellspende war hingegen nur für 57 Prozent und eine Adoption für 48 Prozent eine Option (Hodes-Wertz et al. 2013). Der Wunsch nach einer genetischen Verbindung beschränkt sich jedoch nicht nur auf Mutter und Kind. Die befragten englischen Frauen legen Wert darauf, dass ihr Kind nicht nur eine genetische Verbindung zu innen, sondern auch zu ihrem Partner hat (Baldwin 2016, Waldby 2015). Dies wird darauf zurückgeführt, dass die befragten Frauen eine glück- 
liche Kindheit in einer solchen Familienkonstellation erlebt haben. Daneben scheinen auch die Eltern der befragten Frauen Wert auf eine genetische Verbindung zu ihrem Grosskind zu legen. Einige Frauen wurden von ihren Müttern unterstützt und in die Klinik begleitet. Bei fünf von 15 Frauen übernahm die Familie einen Teil oder alle Kosten der Behandlung (Waldby 2015). Sozial Freezing wird zwar als innovative Technologie gesehen, aber primär zur Aufrechterhaltung traditioneller und naturalisierter Familienbeziehungen genutzt (Waldby 2017, Romain 2011).

\section{Social Freezing für eine Mutterschaft zum richtigen Zeitpunkt}

Social Freezing wird von den Frauen als Möglichkeit erachtet, zum richtigen Zeitpunkt und unter angemessenen Umständen Mutter zu werden (Baldwin 2017). Auch ältere Mütter erklären ihre späte Mutterschaft damit, dass dies der richtige Zeitpunkt für eine Familiengründung war (Daniluk und Koert 2017, Perrier 2013, Budds et al. 2016). Dieser Zeitpunkt wird aber nicht durch das biologische Alter bestimmt, sondern durch biografische Voraussetzungen, welche entscheidend durch Familienleitbilder geprägt werden (Roth 2016).

Das aktuelle Familienleitbild, das auch die Frauen mit kryokonservierten Eizellen prägt, umfasst eine soziale und eine psychologische Komponente. Die soziale Komponente umfasst eine gesicherte Stelle, ein gutes Einkommen sowie eine angemessene Wohnsituation und wird von den englischen Frauen als wichtig erachtet (Baldwin 2016). Die Erfüllung dieser materiellen Voraussetzungen für eine Familiengründung wird ebenfalls in verschiedenen Studien als Erklärung für eine verzögerte Mutterschaft herangezogen (Perrier 2013, Budds et al. 2016). Auch in einer qualitativen Untersuchung des Bundesinstituts für Bevölkerungsforschung im Jahr 2011 sprach sich eine Mehrheit der befragten deutschen Frauen und Männer dafür aus, dass die Familiengründung nach einer abgeschlossenen Ausbildung und einer mehrjährigen Tätigkeit im Beruf erfolgen soll (Roth 2016). Dies spiegelt die normative Vorstellung, dass Familien finanziell vom Staat unabhängig sein sollen (Budds et al. 2016, Roth 2016). Staatliche Leistungen können jedoch auch einen Einfluss darauf haben, ob die materiellen Voraussetzungen für eine Mutterschaft als günstig erachtet werden oder nicht. So war für die englischen Frauen mit kryokonservierten Eizellen beispielsweise ein angemessener Mutterschaftsurlaub am Arbeitsplatz nicht von Bedeutung - im Gegensatz zu sieben amerikanischen Frauen, die Baldwin 
(2017) ebenfalls zur Nutzung des Social Freezing befragte. Dies dürfte darauf zurückzuführen sein, dass in den USA lediglich 12 Prozent der Arbeitnehmer in der Privatwirtschaft Zugang zu einem bezahlten Elternurlaub haben (Martin 2017).

Das geltende Familienleitbild im Westen umfasst auch eine psychologische Komponente. Eine gute Mutter soll ihre eigenen Interessen zurücknehmen, ihr Kind ins Zentrum stellen und sich diesem intensiv widmen können. Diese Ideologie der «intensiven Mutterschaft»(Hays 1996, zitiert in Baldwin 2017) hat gleichzeitig neue normative Vorstellungen von den psychologischen Voraussetzungen für eine Familiengründung begünstigt. So wird eine Phase der Selbstverwirklichung, geprägt durch Ausbildung, Reisen, Freundschaften und Hobbys, als wichtig für die individuelle Entwicklung und emotionale Reife erachtet. Dank dieser Selbstverwirklichungsphase haben die Eltern nicht das Gefühl, etwas verpasst zu haben, wenn sie sich intensiv ihren Kindern widmen (Roth 2016, Baldwin 2017). Diese emotionalen Anforderungen an die Mutterschaft finden sich auch in den Erzählungen der englischen Frauen, die ihre Eizellen kryokonservieren liessen (Baldwin 2017).

Diese normativen Vorstellungen von einer guten Mutterschaft führen dazu, dass sich die Frauen, die ihre Eizellen einfrieren lassen, relativ spät bereit fühlen für eine Familiengründung. Die Technologie erlaubt innen, den sozialen und psychischen Zeithorizont mit ihrer biologischen Uhr in Einklang zu bringen (Baldwin 2017, Romain 2011, Waldby 2015).

\section{Social Freezing als Versicherung gegen zukünftige Unfruchtbarkeit}

In mehreren quantitativen Studien wird berichtet, dass Social Freezing für die Frauen eine Versicherung gegen die künftige Unfruchtbarkeit ist. Für mehr als die Hälfte der Frauen in den zwei amerikanischen Studien war dies ein wichtiger Grund, Social Freezing in Anspruch zu nehmen (Hodes-Wertz et al. 2013, Myers et al. 2015). Eine solche Versicherung trägt dazu bei, dass sich die Frauen künftig nicht vorwerfen müssen, nichts gegen eine abnehmende Fertilität unternommen zu haben. Die Vermeidung von Selbstvorwürfen wurde auch in einer weiteren Studie bei amerikanischen Frauen als wichtigster Grund erwähnt (Witkin et al. 2013). Diese Ergebnisse stammen vor allem aus Befragungen von Kundinnen durch reproduktionsmedizinische Kliniken. Sie beinhalten 
möglicherweise auch eine Verzerrung. Die Resultate entsprechen dem von den Anbietern des Social Freezing gerne genutzten Narrativ, das Frauen mit kryokonservierten Eizellen als vorausschauende und verantwortungsbewusste Frauen beschreibt (Mertes 2013). Allerdings gelangen auch qualitative sozialwissenschaftliche Studien zu ähnlichen Befunden. Die Vermeidung von Selbstvorwürfen in der Zukunft war für ein Drittel der englischen Befragten von Baldwin (2016) ein ausschlaggebender Faktor für die Nutzung des Social Freezing. Social Freezing wird als eine Investition und als eine Art Versicherung für den Fertilitätsverlust beschrieben (Romain 2011).

\subsubsection{Informierter Entscheid für Social Freezing}

Um einen informierten Entscheid für Social Freezing zu fällen, sind Aussagen zu den Erfolgsaussichten dieser Technologie notwendig. Weil nur wenige Frauen bisher auf ihre Eizellen zurückgegriffen haben, bestehen für die Zielgruppe jedoch keine genauen Angaben zu den Erfolgsaussichten. Den Frauen unter 35 Jahren wird empfohlen, 15 bis 20 Eizellen einzufrieren (vgl. Kapitel 2.3.2). Die vorliegenden Befragungen von Frauen deuten darauf hin, dass jedoch teilweise deutlich weniger Eizellen eingefroren wurden. In der australischen Studie wurden bei 24 Prozent der 96 befragten Frauen weniger als acht Eizellen und bei 35 Prozent zwischen acht und 15 Eizellen entnommen (Hammarberg et al. 2017). Bei 8 Prozent der 183 amerikanischen Frauen wurden eine bis fünf Eizellen, bei 28 Prozent fünf bis zehn Eizellen konserviert (Hodes-Wertz et al. 2013). Bei den 23 englischen Frauen wurden im Schnitt 13 Eizellen eingefroren. Die meisten Frauen verfügten über elf bis 16 eingefrorene Eizellen (Baldwin et al. 2015). Da die Mehrheit der Frauen in den zitierten Studien bei der Entnahme der Eizellen deutlich über 35 Jahre alt war, dürfte jedoch eine wesentlich höhere Anzahl Eizellen nötig sein, um eine Lebendgeburt zu erzielen.

In den Artikeln zu den Frauen mit kryokonservierten Eizellen gibt es Hinweise, dass sich die Frauen dieser Grenzen durchaus bewusst sind. So hätten etliche belgische Frauen lieber noch mehr Behandlungszyklen durchlaufen und mit der Entnahme der Eizellen in jüngeren Jahren angefangen (Stoop et al. 2015). Auch die amerikanischen Frauen bedauerten, dass sie die Technologie nicht schon früher genutzt hatten (Hodes-Wertz et al. 2013). Mehrere englische Frauen berichteten, dass sie keine altersspezifische Erfolgsrate erhalten hätten 
und darum die Erfolgsaussichten nicht beurteilen könnten. Die meisten wurden jedoch informiert, dass mehrere Behandlungszyklen notwendig sind und dass es keine Garantie für ein Kind gibt (Baldwin et al. 2015). Die befragten Frauen scheinen die Inanspruchnahme dieser Technologie nicht zu bedauern (Stoop et al. 2015).

\subsubsection{Verwendung der eingefrorenen Eizellen}

In fünf Artikeln wurden die effektive und die geplante Nutzung der eingefrorenen Eizellen beschrieben. Nur wenige Frauen haben bisher auf die eingefrorenen Eizellen zurückgegriffen. In den quantitativen Studien haben keine Frauen (Meyers et al. 2015), 2 Prozent der Frauen (Stoop et al. 2015), 6 Prozent der Frauen (Hodes-Wertz et al. 2013, Hammarberg et al. 2017) respektive 9 Prozent der Frauen (Cabo et al. 2016) ihre eingefrorenen Eizellen genutzt. Von den 23 qualitativ interviewten englischen Frauen haben zwei auf ihre Eizellen zurückgegriffen (Baldwin 2015).

Dass nicht mehr Frauen ihre Eizellen genutzt haben, ist möglicherweise auf den kurzen Zeitabstand zwischen Eizellentnahme und Befragung der Frauen zurückzuführen (Stoop et al. 2015). In der Studie bei 183 amerikanischen Frauen erachteten es 34 Prozent der Befragten als sehr wahrscheinlich und 60 Prozent als eher wahrscheinlich, dass sie später auf die Eizellen zurückgreifen werden (Hodes-Wertz et al. 2013). Bei den australischen Frauen hatten 21 Prozent vor, die Eizellen zu nutzen, während 69 Prozent dies nur unter bestimmten Umständen in Erwägung zogen (Hammarberg et al. 2017). In der belgischen Studie war die Hälfte der Frauen der Ansicht, dass sie ihre Eizellen irgendeinmal nutzen würden (Stoop et al. 2015).

Eine andere Erklärung ist, dass die meisten Frauen selbst in fortgeschrittenem Alter zuerst versuchen, auf natürlichem Weg mit einem Partner schwanger zu werden (Stoop et al. 2015). In der amerikanischen Studie wurden zwei Frauen im Alter von 45 und 47 Jahren auf natürlichem Weg schwanger (Hodes-Wertz et al. 2013). Ohne auf ihre eingefrorenen Eizellen zurückzugreifen, gebaren 16 Prozent der 49 befragten amerikanischen Frauen in der Studie von Meyers et al. (2015) ein Kind. 14 Prozent (Stoop et al. 2015), 18 Prozent (Hodes-Wertz et al. 2013) und 31 Prozent (Hammarberg et al. 2017) der Frauen wurden 
schwanger, ohne ihre Eizellen zu nutzen. In der qualitativen Studie bei englischen Frauen waren es drei von 23. Die gemeinsame Zeugung eines Kindes mit einem Partner wird einerseits als wichtig erachtet, um eine genetische Verbindung zwischen beiden Elternteilen und dem Kind sicherzustellen (Baldwin 2016, Waldby 2015). Andererseits können sich viele der interviewten Frauen auch einige Zeit nach Einfrierung ihrer Eizellen nicht vorstellen, ein Kind allein aufzuziehen, weil innen dazu die finanziellen Mittel respektive die soziale Unterstützung fehlt (Baldwin et al. 2015).

Für etliche Frauen bleibt jedoch die Befruchtung von eigenen frischen oder eingefrorenen Eizellen mit gespendeten Spermien eine letzte Option, wenn die Suche nach einem geeigneten Partner scheitert. In der spanischen Studie liessen 70 der 120 Frauen (58,3\%) die eingefrorenen Eizellen mit den Spermien ihres Partners befruchten, während 50 Frauen $(41,7 \%)$ auf eine Spermienspende zurückgriffen (Cabo et al. 2016). Von den 23 interviewten englischen Frauen wurde eine bereits mit gespendeten Spermien schwanger, zwei waren daran, dank Spermienspende schwanger zu werden, und drei weitere setzten sich gedanklich mit dieser Option vertieft auseinander (Baldwin et al. 2015). Für 52 Prozent der belgischen Frauen stellte die Samenspende für die Befruchtung ihrer eingefrorenen Eizellen eine Alternative dar (Stopp et al. 2015). 63 Prozent der amerikanischen Frauen unter 37 Jahren und 54 Prozent der über 38Jährigen konnten sich vorstellen, eine alleinerziehende Mutter zu werden (Vallejo et al. 2013).

Das Zeitfenster für die Nutzung der Eizellen wird von den Frauen mit kryokonservierten Eizellen nicht als endlos angesehen. Allerdings bestehen diesbezüglich merkliche Unterschiede zwischen den befragten europäischen und amerikanischen Frauen. In der belgischen Studie wollten die Frauen im Schnitt spätestens mit 44 Jahren Mutter werden (Stoop et al. 2015). Von den 23 interviewten englischen Frauen strebten mehrere eine Mutterschaft bis spätestens Ende 40 an (Baldwin et al. 2015). Qualitative Studien zur späten Mutterschaft zeigen, dass die Intergenerationsbeziehung zwischen Grosseltern und Enkelkindern von allen befragten englischen Müttern als wichtig erachtet wird. Die älteren Mütter befürchten, dass ihre Kinder keine Beziehung zu den Grosseltern aufbauen können und dass Letztere die Mütter nicht bei der Kinderbetreuung unterstützen können (Perrier 2013). Auch die interviewten über 40-jährigen Französinnen in reproduktionsmedizinischer Behandlung orientieren sich an bestehenden Familienbildern und möchten die intergenerationellen Beziehun- 
gen aufrechterhalten (Vialle et al. 2016). In den USA konnten sich hingegen 78 Prozent der Frauen vorstellen, im Alter von zwischen 49 und 58 Jahren Mutter zu werden. Für 13 Prozent war gar eine Mutterschaft über 58 Jahren denkbar. Für lediglich 9 Prozent lag die Alterslimite bei zwischen 39 und 48 Jahren (Hodes-Wertz et al. 2013).

Lediglich zwei Artikel thematisieren, was mit überzähligen eingefrorenen Eizellen geschehen soll. Von den 65 befragten belgischen Frauen waren 63 Prozent bereit, ihre nicht verwendeten Eizellen für die Forschung zur Verfügung zu stellen. 18 Prozent sprachen sich für die Vernichtung ungenutzter Eizellen aus. 11 Prozent konnten sich eine Eizellspende vorstellen. Für 8 Prozent kam keine dieser drei Optionen infrage (Stoop et al. 2015). Von den 16 zum Thema befragten englischen Frauen waren sechs bereit, ihre überzähligen Eizellen sowohl für die Forschung als auch als Eizellspende zur Verfügung zu stellen. Fünf konnten sich nur eine Eizellspende vorstellen und drei nur eine Verwendung der Eizellen zu Forschungszwecken. Die relativ hohe Bereitschaft zur Eizellspende wird darauf zurückgeführt, dass viele Befragte von Bekannten mit Zeugungsproblemen berichteten und für deren Leiden sensibilisiert waren (Baldwin et al. 2015).

\subsection{Empirische Befunde zur Nachfrage in der Schweiz}

Dieses Kapitel stützt sich auf Daten aus der Onlinebefragung mit Reproduktionsmedizinerinnen und -medizinern, explorativen Interviews mit vier Reproduktionsmedizinern und zwei Frauen sowie Medienartikel.

\subsubsection{Anzahl Frauen}

Im Rahmen der Onlinebefragung lieferten zehn Fertilitätskliniken Angaben zur Anzahl Frauen, die im Jahr 2017 ihre Eizellen kryokonservieren liessen. Die einzelnen Kliniken behandelten zwischen vier und 42 Frauen. In sechs Kliniken liessen weniger als zehn Frauen ihre Eizellen einfrieren. Insgesamt wurden im 
Jahr 2017 in den zehn Kliniken 139 Frauen behandelt. Da in der Stichprobe Universitätsspitäler, Kantonsspitäler, private Kliniken, alle Landesteile sowie grössere und kleinere Anbieter vertreten waren, scheint ein relativ repräsentativer Ausschnitt der 30 Schweizer Fertilitätskliniken an der Onlinebefragung teilgenommen zu haben. Grob geschätzt, haben im Jahr 2017 in der Schweiz wahrscheinlich rund 400 Frauen ihre Eizellen kryokonservieren lassen. Diese Zahl entspricht auch in etwa unserer Schätzung, gestützt auf die Onlinebefragung bei kinderlosen Frauen zwischen 25 und 39 Jahren. Ein Prozent der an dieser Umfrage teilnehmenden Frauen hat ihre Eizellen bereits kryokonservieren lassen. Dies entspricht rund 300 Frauen jährlich (vgl. Kapitel 4.2.6).

Die interviewten Reproduktionsmediziner berichten von einer steigenden Nachfrage. In einer der Kliniken hat sich die Nachfrage zwischen 2014 und 2017 vervierfacht. Auch in den untersuchten Schweizer Medien wird über eine steigende Nachfrage in den letzten Jahren berichtet.

"Auch in der Schweiz stellen Ärzte ein immer grösseres Interesse an Social Freezing fest. Am Unispital Zürich verzeichnet man eine langsame Steigerung der Reproduktionsmethode. «Pro Jahr werden Eingriffe im tiefen zweistelligen Bereich durchgeführt), sagt Sprecherin Katrin Hürlimann zu ‘20 Minuten`. Im Luzerner Kantonsspital führt man ebenfalls mehr solcher Eingriffe durch, wie es auf Anfrage heisst. Deutlich spürt den Boom das $360^{\circ}$-Kinderwunsch-Zentrum in Zürich, wie Florian Götze, Facharzt für Gynäkologie und Geburtshilfe, sagt: ‘lm Vergleich zu 2014 führen bei uns heute zwischen 30 und 40 Prozent mehr Patientinnen ein Social Freezing durch.ı» (20 Minuten, 2017)

Die interviewten Reproduktionsmediziner gehen davon aus, dass die Nachfrage weiter zunehmen wird. Treibende Kräfte seien das laufend steigende Alter der Erstgebärenden sowie eine positivere Haltung gegenüber der Reproduktionsmedizin insbesondere bei den jüngeren Generationen. Auch die Versicherungsmentalität spiele eine Rolle. In den Medien wird diese Ansicht ebenfalls vertreten. Allerdings werden auch ambivalente Expertinnen und Experten zitiert, die davon ausgehen, dass sich Social Freezing kaum zu einem Massenphänomen oder Standardverfahren entwickeln werde und die "grosse Mode» bereits am Abklingen sei. Dies wird vor allem damit begründet, weil die meisten interessierten Frauen bereits über 35 Jahre alt seien, die Erfolgsaussichten entsprechend klein seien und nicht im Verhältnis mit dem entsprechenden Aufwand stehen würden. 


\subsubsection{Soziodemografische Merkmale der Frauen}

Die soziodemografischen Merkmale der Frauen, die ihre Eizellen in der Schweiz einfrieren lassen, unterscheiden sich nicht von denjenigen im Ausland. Nach übereinstimmender Einschätzung der interviewten respektive in den Medien zitierten Reproduktionsmedizinerinnen und -mediziner handelt es sich dabei um gut ausgebildete und gut verdienende Frauen Mitte 30. Die Frauen sind bei Banken, in der Steuerberatung und in IT-Unternehmen wie Google tätig oder arbeiten als IKRK-Delegierte. Ein Reproduktionsmediziner gab an, dass seine Kundinnen primär aus der Schweiz stammen, während die beiden anderen auch Expats respektive Frauen aus dem Ausland zu ihren Kundinnen zählen. Die beiden interviewten Frauen waren ebenfalls fast 35 und gut situiert. Nach Aussage der interviewten Reproduktionsmediziner verzeichnen Kliniken im urbanen Raum eine stärkere Nachfrage als solche in eher ländlichen Regionen.

Wie viele Frauen ins Ausland reisen, um ihre Eizellen kryokonservieren zu lassen, ist unbekannt. Die interviewten Reproduktionsmediziner vermuten, dass die Kosten nicht ausschlaggebend sein dürften, sondern eher die rechtlichen Rahmenbedingungen. Alleinstehende Frauen auf der Suche nach einem Spermienspender würden nach Spanien überwiesen. Eine der interviewten Frauen hatte sich bei einer Klinik in Spanien über Social Freezing informiert, aber eine Behandlung im Ausland als umständlicher erachtet als in einer Klinik gleich um die Ecke. Hätte es aber die Möglichkeit nicht gegeben, die eingefrorenen Eizellen nach Ablauf der Lagerungsfrist ins Ausland zu transportieren, hätte sie sich nicht in der Schweiz behandeln lassen.

\subsubsection{Motivation der Frauen}

Auch hier zeigen sich keine wesentlichen Unterschiede zu den Studien aus dem Ausland. Gemäss den interviewten Reproduktionsmedizinern liegt es hauptsächlich am fehlenden Partner, dass Frauen auf Social Freezing zurückgreifen. Ein interviewter Reproduktionsmediziner schätzt, dass dies sicher bei zwei Dritteln der Frauen zutrifft. Ein Drittel lebe aber bereits in einer festen Partnerschaft und möchte mehr Zeit, um sich beruflich zu entwickeln oder zu reisen. Die in den Medien zitierten Reproduktionsmedizinerinnen und -medi- 
ziner betonen ebenfalls, dass nicht Karrieregründe das Hauptmotiv für Social Freezing seien, sondern der fehlende Partner.

"Überwiegend sind es aber Frauen, die aktuell keinen Partner haben und deshalb wahrscheinlich auch noch gar nicht probiert haben, auf natürlichem Weg schwanger zu werden. Das Paradebeispiel, das man sich immer vorstellt, nämlich die knallharte Karrierefrau, die an einer Eliteuniversität studiert hat und mit 30 ihre Eizellen einfrieren lässt, damit sie in aller Ruhe noch das grosse Geld verdienen kann, also dieses Paradebeispiel ist wohl eher ein Fantasiekonstrukt.» (www.wireltern.ch, Erscheinungsdatum unbekannt, M. von Wolff - Reproduktionsmediziner)

Für beide interviewten Frauen war der fehlende Partner der Hauptgrund, ihre Eizellen kryokonservieren zu lassen. Eine der Frauen hat sich nach ihrer Scheidung für Social Freezing entschieden, um wieder mehr Kontrolle über ihr Leben zu haben. Im Gegensatz zu ihrem Ex-Mann hatte sie einen Kinderwunsch und spürte den Druck der biologischen Uhr. Auch die zweite Frau verspürte einen grossen Kinderwunsch, hatte aber keinen Partner. Sie wünschte sich nicht nur ein Kind, sondern eine Familie mit einem Partner, der für sie wie ein bester Freund sein sollte. Irgendein Mann kam für sie nicht infrage: «lch weiss, gewisse Frauen lassen sich einfach schwängern. Aber für mich ist das keine Option.» Aber auch aus finanziellen Gründen kann sie sich nicht vorstellen, ein Kind allein zu erziehen. Beide Frauen berichten übereinstimmend, dass Social Freezing den Druck aus der Partnersuche genommen habe. Sie bemerkten, dass sie ohne das Ticken der biologischen Uhr nun viel entspannter auftreten würden als vorher und die Männer positiv darauf reagierten.

In den Aussagen der Frauen taucht auch der Wunsch nach einer genetischen Verbindung zum Kind auf. Eine der beiden schliesst zwar eine Adoption nicht aus, möchte aber auch die Aussicht auf ein eigenes Kind aufrechterhalten. Für die andere Frau wäre eine Adoption keine Alternative. Sie möchte, dass ihr Kind genetisch verwandt mit ihr ist. Die Gründung einer Familie sei ihr zwar sehr wichtig, aber nicht unter jeder Bedingung.

Ebenso ist in der Aussage einer Frau die Motivation erkennbar, alles Mögliche zur Erfüllung des Kinderwunsches getan zu haben. Falls es nicht klappt, hat sie sich nichts vorzuwerfen. Social Freezing ist eine Art «Backup-Plan». 
Karrieremotive spielen bei beiden Frauen hingegen keine Rolle. Eine der interviewten Frauen wehrt sich gegen das in den Medien präsentierte Stereotyp der «Karrierefrau», die primär auf Social Freezing zurückgreift.

\subsubsection{Informierter Entscheid}

Gemäss den Aussagen eines interviewten Reproduktionsmediziners lassen zwei Drittel der Frauen lediglich zehn Eizellen einfrieren. Möglicherweise haben sie dadurch reduzierte Aussichten auf ein Kind (vgl. Kapitel 2.4.3). Aufgrund der vorliegenden Angaben kann nicht beurteilt werden, wie gut informiert dieser Entscheid ist und ob primär Kostengründe ausschlaggebend sind. Die beiden interviewten Frauen scheinen genügend Eizellen eingefroren zu haben. Sie bereuen ihren Entscheid für Social Freezing nicht und würden die Technologie anderen Frauen weiterempfehlen. Allerdings scheint zumindest eine der beiden nicht sehr gut über die Risiken der Technologie für die Kinder informiert zu sein (vgl. Kapitel 2.4.2).

\subsubsection{Verwendung der Eizellen}

Bisher hat praktisch noch keine Frau auf ihre Eizellen zurückgegriffen. Ein interviewter Reproduktionsmediziner berichtete von einer Frau, die zwei Jahre nach der Kryokonservierung ihre Eizellen in eine andere Schweizer Klinik transferieren und dort in vitro befruchten liess.

Die beiden interviewten Frauen möchten spätestens Anfang 40 auf ihre Eizellen zurückgreifen. Idealerweise erfolgt die Befruchtung in vitro mit den Spermien eines Partners. Für eine der beiden Frauen wäre noch eine Adoption denkbar und danach eine Samenspende. Die andere Frau könnte sich eine Samenspende vorstellen, aber nur, wenn sie das Kind gemeinsam mit einer weiteren Person, zum Beispiel ihrem homosexuellen Bruder, grossziehen könnte. Eine Adoption kommt für sie nicht infrage. 
Über die Verwendung der eingefrorenen Eizellen haben sich beide noch keine Gedanken gemacht. Einer der beiden wäre es lieber, wenn die Eizellen nicht weggeworfen würden, sondern der Forschung oder nach ihrem Tod einer unfruchtbaren Frau zur Verfügung gestellt werden könnten. Solange sie lebt, möchte sie nicht mit der Vorstellung konfrontiert sein, dass irgendwo noch ein Kind von ihr existiert. Auch für die zweite Frau wäre eine Eizellspende gefühlsmässig so, als würde sie ihre eigenen Kinder weggeben, und daher unvorstellbar. 


\section{Potenzielles Interesse am Social Freezing}

\subsection{Internationale Studien zum potenziellen Interesse am Social Freezing}

Während in der Schweiz bisher keine Daten zum potenziellen Interesse am Social Freezing erhoben wurden, existieren dazu Studien aus verschiedenen europäischen und insbesondere nordamerikanischen Ländern. Die grosse Mehrheit dieser Studien erfolgte gestützt auf eine anonymisierte Onlinebefragung. Die Studien unterscheiden sich jedoch bezüglich untersuchter Populationen. So nahmen an einer Studie ausschliesslich (Meissner et al. 2016) und an einer Studie hauptsächlich Studentinnen teil (Gay et al. 2013). Während die meisten Studien auch Teilnehmende mit Kindern einschliessen, fokussieren sich vier Studien auf kinderlose Frauen (Daniluk et al. 2012, Stanton et al. 2014, Keurst et al. 2016, Daniluk et al. 2016). An einer kanadischen Studie und zwei deutschen Befragungen beteiligten sich auch Männer (Daniluk et al. 2012, Schick et al. 2017, Meissner et al. 2016). Das Alter der Teilnehmenden variiert ebenfalls zwischen den Studien. Eine Studie in mehreren angelsächsischen Ländern (Keurst et al. 2016) fokussiert sich auf die Hauptzielgruppe des Social Freezing, die 28- bis 35-Jährigen. Andere Studien umfassen eine grössere Altersspanne und schliessen auch Frauen nach der Menopause mit ein (Gay et al. 2013, Daniluk et al. 2012). Auch die Zahl der Befragten sowie die Strategien zur Rekrutierung der Teilnehmenden variieren beträchtlich. Meistens wurden die Teilnehmenden über verschiedene Kanäle auf die Studien hingewiesen und haben aus eigenem Antrieb an der Befragung teilgenommen. Die Ergebnisse sind entsprechend nicht repräsentativ. In zwei kanadischen Studien wurden Daten aus einem Register einer privaten Firma (Daniluk et al. 2012) und in einer belgischen Studie aus einem nationalen Register (Stoop et al. 2011) genutzt, um eine möglichst geografisch und sozial repräsentative Stichprobe von Teilnehmerinnen anzuschreiben. Eine Studie wird als repräsentativ beschrieben (Milman et al. 2017). 


\subsubsection{Wissen zum Social Freezing}

Gemäss sechs Studien ist eine Mehrheit der Befragten über die Möglichkeit informiert, die eigenen Eizellen einfrieren zu lassen. Die Zahlen variieren zwischen 55 Prozent der befragten Studentinnen an Hochschulen in Hannover (Meissner et al. 2016) und 93 Prozent bei einer Befragung in den USA von Frauen zwischen 20 und 56 Jahren (Stanton et al. 2014). Da die Teilnahme an der Befragung in fünf Studien (Gay et al. 2013, Lallemant et al. 2016, Meissner et al. 2016, Milman et al. 2017, Stanton et al. 2014) aus eigenem Antrieb erfolgte, dürften das Interesse und der Kenntnisstand der Befragten höher sein als in der Gesamtbevölkerung. Verlässlichere Daten liefert die Studie aus Belgien, in der eine soziale und geografische Stichprobe von Frauen aus einem nationalen Register angeschrieben wurde und die einen Rücklauf von 55 Prozent aufweist (Stoop et al. 2011). In dieser Studie gaben 78 Prozent der Frauen an, bereits von Social Freezing gehört zu haben.

\subsubsection{Einstellung zum Social Freezing}

In den meisten Studien äusserte nur eine Minderheit der Befragten ein potenzielles Interesse, Social Freezing vorzunehmen. In der repräsentativen belgischen Studie bei 1914 Frauen zwischen 21 und 40 Jahren würden 28 Prozent vielleicht Social Freezing in Anspruch nehmen (Stoop et al. 2011). Zu einem ähnlichen Befund gelangte die repräsentative Befragung in den USA, in der 25 Prozent der Teilnehmenden zwischen 20 und 45 Jahren ein Interesse an der Technologie äusserten. Bei kinderlosen Frauen zwischen 20 und 50 Jahren in einer repräsentativen kanadischen Studie waren es 35 Prozent. In dieser Studie äusserten zudem 44 Prozent der Männer ein Interesse daran, ihre Spermien einfrieren zu lassen (Daniluk et al. 2012). Die Ergebnisse in den nicht repräsentativen Studien bewegen sich in einem ähnlichen Rahmen. In einer Befragung von 1144 Studierenden in Hannover zeigten 24 Prozent der Befragten ein grundsätzliches Interesse am Social Freezing (Meissner et al. 2016). Eine weitere deutsche Studie hält fest, dass 36 Prozent der Frauen eine positive Einstellung gegenüber Social Freezing haben (Schick et al. 2017). Eine Befragung von 973 dänischen und englischen Frauen ergab ein potenzielles Interesse von 27 Prozent der Befragten (Lallemant et al. 2016). 
Ein konkretes Interesse besteht jedoch bei deutlich weniger Befragten. So würden in der repräsentativen Studie bei belgischen Frauen lediglich 3 Prozent sicher Social Freezing in Anspruch nehmen (Stoop et al. 2011). Eine nicht repräsentative Studie in den USA, Kanada und Grossbritannien fokussierte sich auf kinderlose Frauen ohne Fertilitätsprobleme im Alter zwischen 28 und 35 Jahren. In dieser Hauptzielgruppe des Social Freezing hatten lediglich 5 Prozent der 257 Befragten einen Entscheid betreffend Social Freezing gefällt, während sich 85 Prozent nicht in einem Entscheidungsprozess befanden (Keurst et al. 2016). In den USA haben sich 7 Prozent der 200 kinderlosen, alleinstehenden, im urbanen Raum wohnhaften Frauen bereits vertieft mit Social Freezing befasst (Stanton et al. 2014). Von den 24 Prozent der Studierenden in Hannover, die sich für Social Freezing interessieren, würden lediglich 3 Prozent sofort zusätzliche Informationen wünschen, während Social Freezing für 10 Prozent erst ein Thema in fünf und für 11 Prozent erst in zehn Jahren wäre (Meissner et al. 2016). Bei den befragten dänischen und englischen Frauen scheint das aktive Interesse mit 19 Prozent grösser zu sein (Lallemant et al. 2016).

\subsubsection{Merkmale der potenziell interessierten Frauen}

Gewisse Merkmale zeichnen die am Social Freezing potenziell interessierten Frauen gemäss den untersuchten Studien aus.

- Potenziell Interessierte sind jünger: In drei Studien wird berichtet, dass die am Social Freezing interessierten befragten Frauen im Durchschnitt eher jünger sind als die nicht interessierten Frauen (Stoop et al. 2011, Gay et al. 2013, Lallemant et al. 2016). Dies dürfte darauf zurückzuführen sein, dass die erwähnten Studien zum potenziellen Interesse auch ältere Frauen mit Kindern einschlossen, welche die Familienplanung bereits abgeschlossen hatten und Social Freezing aus diesem Grund nicht mehr in Erwägung zogen.

- Potenziell Interessierte sind alleinstehend: Die eher am Social Freezing interessierten Frauen sind weniger oft verheiratet (Stoop et al. 2011) beziehungsweise Singles (Lallemant et al. 2016, Milman et al. 2017) als nicht interessierte Frauen. 
- Potenziell Interessierte haben einen stärkeren Kinderwunsch: Gemäss einer deutschen Studie war ein aktuell bestehender beziehungsweise ein genereller Kinderwunsch der wichtigste Grund für eine positive Einstellung gegenüber Social Freezing (Schick et al. 2017). Am Social Freezing Interessierte scheinen sich zudem mehr Kinder zu wünschen als Nichtinteressierte (Stoop et al. 2011).

- Potenziell Interessierte befürworten eine späte Mutterschaft: Am Social Freezing interessierte Frauen können sich eher vorstellen, in fortgeschrittenem Alter Mutter zu werden, als nicht an dieser Technologie interessierte Befragte (Keurst et al. 2016, Stoop et al. 2011).

- Potenziell Interessierte leiden unter Infertilität oder befürchten diese: Befürworterinnen des Social Freezing haben in der Vergangenheit eher unter Fertilitätsproblemen gelitten (Schick et al. 2017, Lallemant et al. 2016, Milman et al. 2017). Die Befragten sprechen sich zudem eher für Social Freezing aus, wenn sie Fertilitätsprobleme erwarten oder sehr stark unter einer Infertilität leiden (Keurst et al. 2016).

\subsubsection{Entscheidungskriterien}

Die Studien untersuchten zudem, welche Kriterien die befragten Frauen in einen Entscheid für Social Freezing miteinbeziehen würden.

- Komplexität des Verfahrens: Für 58 Prozent der befragten belgischen Frauen war die Aussicht auf eine wenig komplexe Behandlung und eine Behandlung in der Nähe ein Entscheidungskriterium (Stoop et al. 2011). Für 45 Prozent der befragten deutschen Frauen wäre ein geringer organisatorischer Aufwand wichtig (Schick et al. 2017).

- Gesundheitliche Risiken für die Frau: Für 75 Prozent der befragten belgischen Frauen (Stoop et al. 2011), 84 Prozent der deutschen Frauen (Schick et al. 2017) sowie die dänischen und englischen Frauen war es von Bedeutung (Lallemant et al. 2016), dass sich Social Freezing nicht negativ auf ihre künftige Fertilität auswirkt. Für 86 Prozent der befragten kanadischen kinderlosen Frauen war es zudem wichtig, dass die Technologie 
nicht mit einem gesundheitlichen Risiko für sie selber verbunden ist (Daniluk et al. 2016).

- Gesundheitliche Risiken für das Kind: Social Freezing soll zudem für 71 Prozent der belgischen (Stoop et al. 2011) und 90 Prozent der befragten deutschen Frauen (Schick et al. 2017) zu einem gesunden Kind führen.

- Erfolgsaussichten: Für 62 Prozent der befragten belgischen Frauen (Stoop et al. 2011), 82 Prozent der kanadischen Frauen (Daniluk et al. 2016) und 74 Prozent der befragten deutschen Frauen (Schick et al. 2017) waren bessere Erfolgsaussichten ein wichtiges Kriterium. Für die dänischen und englischen Frauen wäre es wichtig, dass die Erfolgschancen über 50 Prozent liegen (Lallemant et al. 2016).

- Kosten: Für 66 Prozent der befragten belgischen Frauen (Stoop et al. 2011), 86 Prozent der kanadischen Frauen (Daniluk et al. 2016) und 74 Prozent der befragten deutschen Frauen (Schick et al. 2017) waren die Kosten beziehungsweise eine Kostenbeteiligung durch Dritte von Bedeutung. Für die englischen und dänischen Frauen scheinen die Kosten keine wichtige Einflussgrösse zu sein (Lallemant et al. 2016). Eine amerikanische Studie zeigt eine Zahlungsbereitschaft von 3812 Dollar für Social Freezing. Die Befragten wären jedoch bereit, bis zu 10'000 Dollar zu zahlen, wenn die Erfolgsaussichten bei 50 Prozent liegen würden (Milman et al. 2017).

Wie sich diese Entscheidungskriterien konkret auf das potenzielle Interesse am Social Freezing auswirken, wurde in den meisten Studien nicht untersucht. Zwei amerikanische Studien liefern jedoch Hinweise auf ein Zusammenspiel der erwähnten Entscheidungskriterien. So änderten 30 Prozent der potenziell am Social Freezing interessierten Befragten ihre Ansicht, nachdem innen das Vorgehen zur Entnahme der Eizelle erläutert wurde (Milman et al. 2017). In einer anderen Studie stieg das potenzielle Interesse von 39 auf 60 Prozent, nachdem die Befragten auf die abnehmende Fertilität hingewiesen worden waren (Gay et al. 2013). Verschiedene Studien zeigen, dass Frauen die voranschreitende Unfruchtbarkeit unterschätzen und gleichzeitig die Möglichkeiten der IVF im Alter überschätzen (Peter 2015). 


\subsection{Empirische Befunde zum potenziellen Interesse am Social Freezing in der Schweiz}

In diesem Kapitel werden die Erkenntnisse aus der Onlinebefragung bei kinderlosen Frauen zwischen 25 und 39 Jahren beschrieben.

\subsection{1}

Kenntnis und Informationsquellen zum Social Freezing

Die Befragung der kinderlosen Frauen zwischen 25 und 39 Jahren zeigt, dass knapp die Hälfte der Befragten (48 \%) schon einmal von Social Freezing gehört hat. Dies deutet darauf hin, dass in der Schweiz die Kenntnis über Social Freezing weniger verbreitet ist als in anderen Ländern, in denen entsprechende Umfrageergebnisse vorliegen (vgl. Kapitel 4.1, Internationale Studien).

In der Westschweiz kennen knapp ein Drittel (31\%) der kinderlosen Frauen das Verfahren, in der Deutschschweiz sind es etwas mehr als die Hälfte (54\%); der Unterschied ist statistisch signifikant. ${ }^{10}$ Frauen mit höherer Bildung kennen das Verfahren tendenziell eher: Bei den Frauen mit Bachelor- oder Masterabschluss oder Eidgenössischem Fähigkeitszeugnis sind es über die Hälfte (52\% und $51 \%$ ), bei den Frauen mit Lehrabschluss oder Maturität sind es 38 Prozent und 42 Prozent. Allerdings kennen auch 55 Prozent der Frauen, bei denen der höchste Bildungsabschluss die obligatorische Schulbildung ist, das Verfahren, wobei die Fallzahl mit elf Personen hier sehr gering ist. Die Unterschiede in der Bildung in Bezug auf die Bekanntheit des Social Freezing sind zudem nicht statistisch signifikant. Auch keine signifikanten Unterschiede lassen sich beim Alter, beim Einkommen und bei der Lebenssituation feststellen (Single, mit

Zur Analyse, ob Unterschiede zwischen Gruppen oder Antworten signifikant sind, wurde ein Chi-Quadrat-Test verwendet. Dieser Test, angewandt auf Kreuztabellen, ermittelt die Wahrscheinlichkeit, ob Zusammenhänge mehr als nur zufälliger Natur sind. In der Regel wird dafür der Wert 0.05 oder 0.01 verwendet, für diese Analyse wurden beide Werte getestet. Ist im Text von «signifikant» die Rede, bedeutet dies, dass mit einer Wahrscheinlichkeit von mehr als 95 Prozent anzunehmen beziehungsweise mit einer Irrtumswahrscheinlichkeit von weniger als 5 Prozent anzunehmen ist, dass tatsächlich ein Zusammenhang zwischen zwei Ausprägungen oder Aussagen besteht. 
Partner oder verheiratet). Nachfolgende Abbildung 7 fasst die Ergebnisse zu den Erkenntnissen über die Bekanntheit des Social Freezing bezogen auf soziodemografische Merkmale zusammen.

Abbildung 7: Kenntnis über Social Freezing nach soziodemografischen Variablen, in Prozent

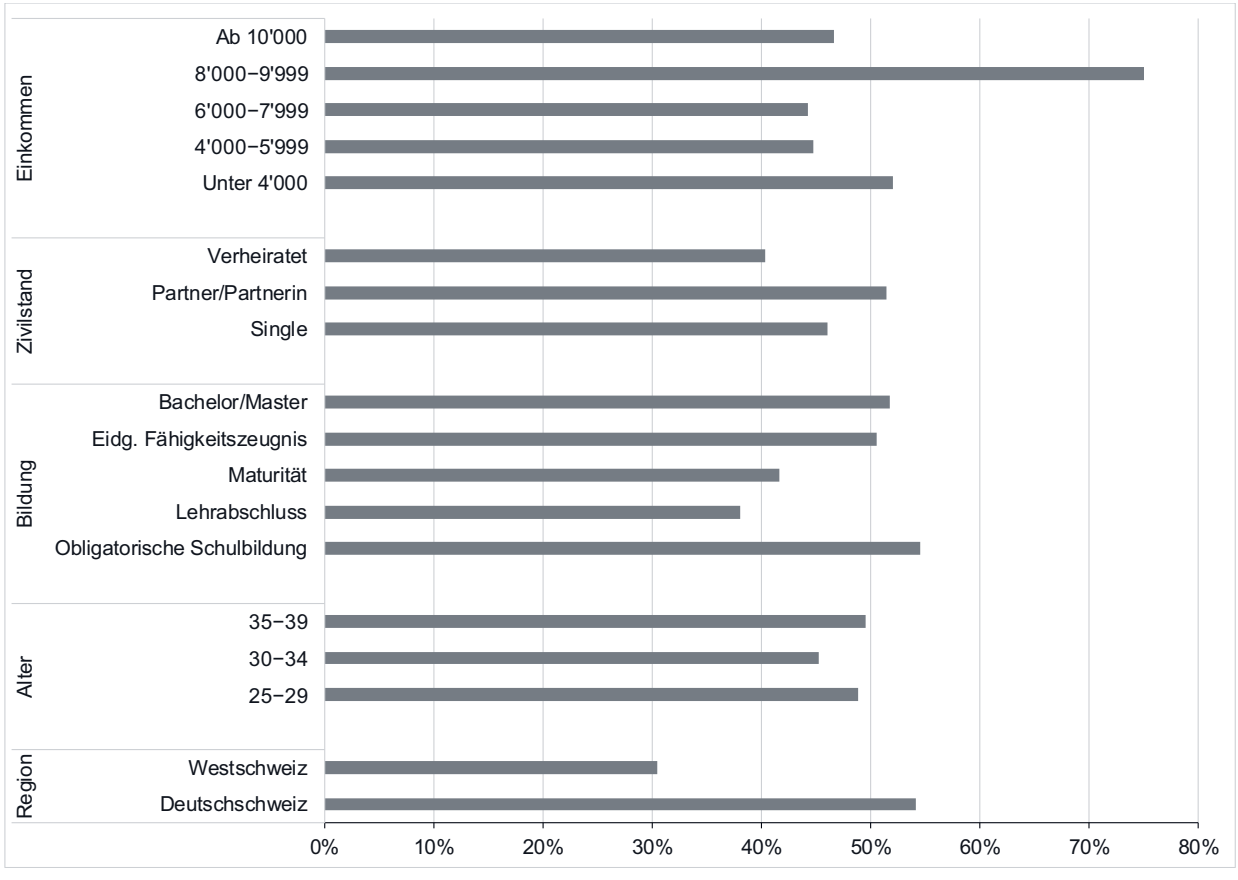

Quelle: Befragung Social Freezing 2018, $n=408$ für Region und Alter, $n=406$ für Bildung, $n=399$ für Lebenssituation und $n=333$ für Einkommen, da nicht alle Befragten hier Angaben gemacht haben.

Als häufigste Informationsquelle zum Social Freezing werden TV-Medien angegeben (53\%), gefolgt von Zeitungen/Zeitschriften (38\%), dem Bekanntenkreis $(27 \%)$ und «anderes» (9\%). Unter "anderes» wurden insbesondere das Internet und das Studium genannt. Weiter wurden von wenigen Frauen die Homepage/Broschüre einer Klinik (7 \%) und der Frauenarzt (3 \%) als Quelle genannt. 


\subsubsection{Interesse am Social Freezing}

Die Frauen wurden im Rahmen der Befragung zu verschiedenen Zeitpunkten zu ihrem Interesse am Social Freezing befragt.

\section{Interesse am Social Freezing ohne zusätzliche Informationen}

Ohne nähere Informationen zur Technologie erhalten zu haben, können 40 Prozent der Befragten sich vorstellen, ihre Eizellen einfrieren zu lassen, oder haben dies bereits getan ( $1 \%$ ); 7 Prozent wissen es nicht oder machen keine Angaben bei dieser Frage, die restlichen 52 Prozent können es sich nicht vorstellen. Ein Anteil von 40 Prozent an Interessierten ist etwas höher als im internationalen Vergleich (vgl. Kapitel internationale Studien). Allerdings sind die Ergebnisse nicht direkt vergleichbar, weil die Stichprobe der befragten Frauen bezüglich Alter und Lebenssituation (kinderlos/mit Kind) variiert.

Danach erhielten die Frauen in der Onlinebefragung einerseits zusätzliche Informationen zur abnehmenden Fruchtbarkeit der Frau und andererseits vertiefte Informationen zur Technologie. Wie sich diese Informationen auf das Interesse am Social Freezing auswirkten, wird in der Folge beschrieben.

Einfluss zusätzlicher Informationen zur abnehmenden Fruchtbarkeit auf das Interesse am Social Freezing

Eine grosse Mehrheit der befragten Frauen weiss, dass die Fruchtbarkeit bei Frauen ab 35 schnell abnimmt (87\%). 91 Prozent sind sich zudem bewusst, dass mit 45 Jahren kaum noch eine Frau Mutter wird. Für relativ viele Frauen ist allerdings neu, dass die Reproduktionsmedizin die Aussichten auf ein Kind bei über 40-Jährigen nicht verbessern kann. Lediglich 47 Prozent der Frauen waren über diese Tatsache im Bilde. Von den Frauen, für die eine oder mehrere dieser Informationen neu waren und die zuvor angegeben hatten, dass sie es sich nicht vorstellen könnten, Social Freezing in Anspruch zu nehmen $(n=109)$, gaben lediglich 3 Prozent danach an, sich nun eher vorstellen zu können, ihre Eizellen einfrieren zu lassen. 
Einfluss zusätzlicher Informationen zur Technologie auf das Interesse am Social Freezing

Die Frauen wurden in der Umfrage gebeten, anzugeben, wie sich detaillierte Informationen zum Verfahren, zu den Risiken für Mutter und Kind, zu den Erfolgsaussichten, zu den Kosten sowie zur Rechtslage auf ihre Einstellung gegenüber Social Freezing auswirken (vgl. Abbildung 8).

- Die verschiedenen Detailinformationen erschweren für einen beachtlichen Anteil der befragten Frauen den Entscheid, Social Freezing zu beanspruchen. Detaillierte Informationen zu den Kosten (59\%), gefolgt von denjenigen zu den Risiken für die Kinder (50\%) und denjenigen für die Frauen (46\%) beeinflussen die Frauen am meisten negativ in ihrem Entscheid, Social Freezing zu beanspruchen. Am geringsten ist die negative Beeinflussung bei den Informationen zum Verfahren $(28 \%)$.

- Detaillierte Informationen zum Verfahren und zur Rechtslage in der Schweiz haben den geringsten Einfluss auf den Entscheid für oder gegen die Inanspruchnahme des Social Freezing. Jeweils mehr als 50 Prozent bleiben von diesen Informationen unbeeinflusst.

- Nur jeweils wenige Frauen werden durch die detaillierten Informationen positiv beeinflusst, Social Freezing zu beanspruchen. 14 Prozent geben an, dass innen Informationen über die Erfolgsaussichten den Entscheid für Social Freezing erleichtern würden. Immerhin 10 Prozent geben an, dass innen Informationen zum Verfahren den Entscheid für Social Freezing erleichtern würden. Bei den übrigen Informationen werden zwischen 3 und 6 Prozent der Befragten positiv beeinflusst.

Die Frauen, die bei den Informationen zur aktuellen Rechtslage geantwortet haben, dass diese ihren Entscheid nicht oder negativ beeinflussen würden, wurden zusätzlich gefragt, wie ihr Entscheid bei einer veränderten Rechtslage ausfallen würde (hier waren Mehrfachantworten möglich, $n=394$ ). 55 Prozent dieser Frauen geben an, dass sie sich Social Freezing auch bei einer anderen Rechtslage nicht vorstellen könnten. 20 Prozent könnten sich Social Freezing vorstellen, wenn alleinstehende Frauen Zugang zur Befruchtung in vitro hätten, 
und 19 Prozent, wenn homosexuelle Paare Zugang zur Befruchtung in vitro hätten. 8 Prozent könnten sich Social Freezing vorstellen, wenn die Eizellen mehr als zehn Jahre gelagert werden dürften.

Abbildung 8: $\quad$ Beeinflussung der Einstellung zum Social Freezing durch Informationen, in Prozent

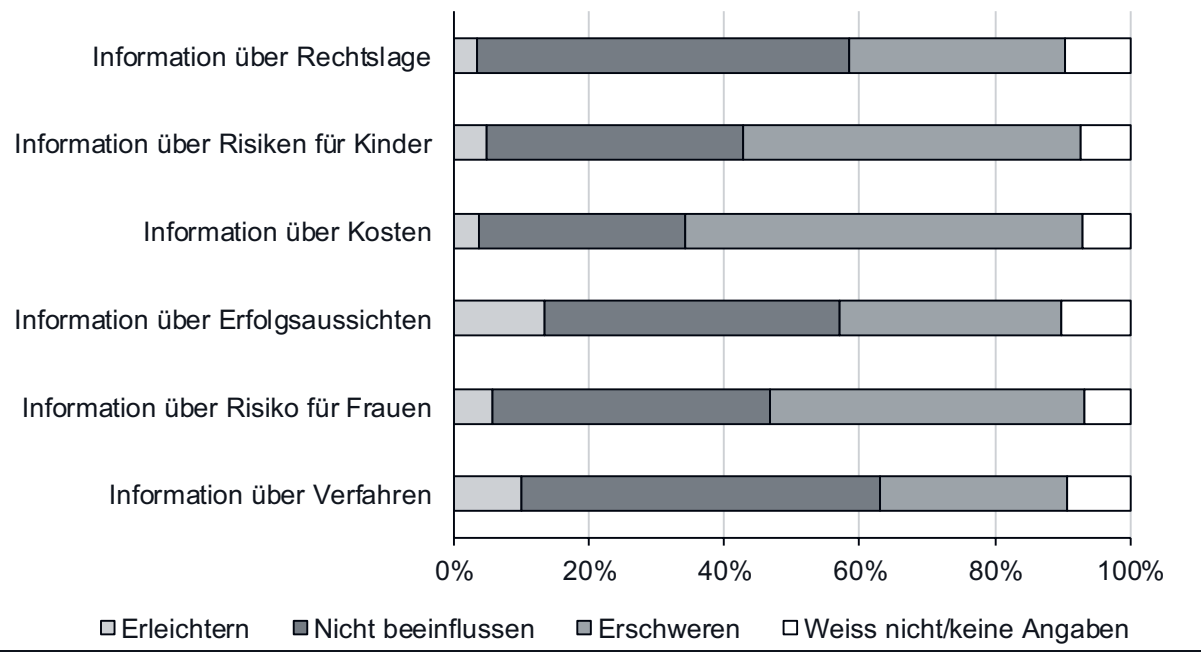

Quelle: Befragung Social Freezing 2018; $n=408$.

\section{Interesse am Social Freezing nach zusätzlicher Information}

Nachdem die Frauen detaillierte Zusatzinformationen zum Verfahren, zu den Risiken für Mutter und Kind, den Erfolgsaussichten, den Kosten sowie der Rechtslage erhalten haben, beträgt der Anteil Frauen, der sich vorstellen könnte, die Eizellen einfrieren zu lassen, noch 34 Prozent $(n=404)$. 57 Prozent können sich dies nach detaillierten Informationen nicht (mehr) vorstellen und 9 Prozent wissen es nicht oder beantworten die Frage nicht (siehe Abbildung 9).

Hinsichtlich des Interesses am Social Freezing nach Erhalt zusätzlicher Informationen gibt es signifikante Unterschiede zwischen den Alterskategorien: Das Interesse am Social Freezing ist mit 38 beziehungsweise 35 Prozent höher bei den beiden jüngeren Altersgruppen (25- bis 29-Jährige und 30- bis 34-Jährige) 
als bei den 35 - bis 39-Jährigen (25\%). Hinsichtlich Bildungsabschluss, Sprachregion; Einkommen und Lebenssituation (Single, mit Partner oder verheiratet) gibt es keine signifikanten Unterschiede.

Abbildung 9: $\quad$ Interesse am Social Freezing: Könnten Sie sich grundsätzlich vorstellen, Ihre Eizellen vorsorglich einfrieren zu lassen?

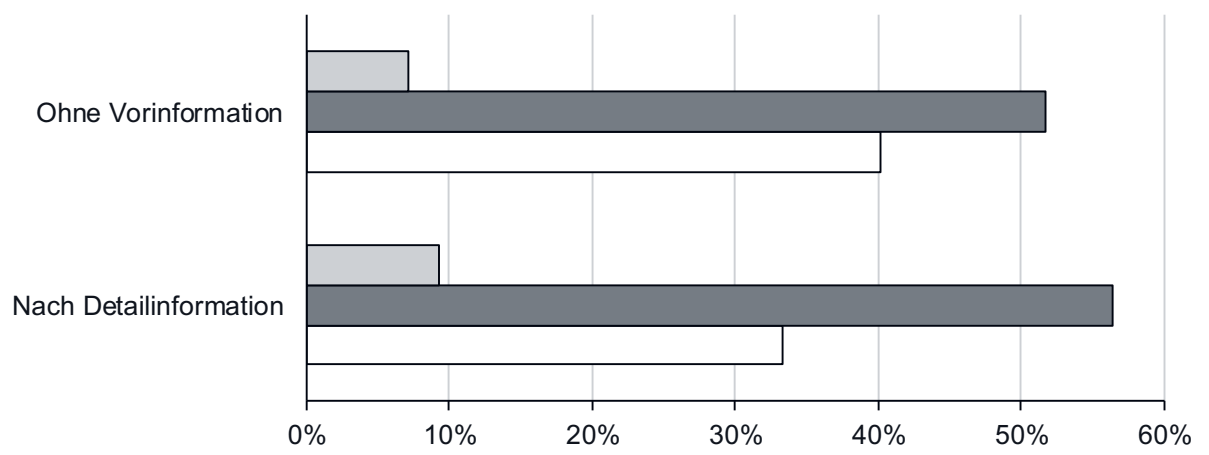

$\square$ Weiss nicht / keine Antwort

口 Könnte es mir grundsätzlich nicht vorstellen

$\square$ Könnte es mir grundsätzlich vorstellen

Quelle: Befragung Social Freezing 2018; $n=408$.

\subsubsection{Motivation für die Inanspruchnahme des Social Freezing}

Die Frauen wurden gefragt, welches für sie die wichtigsten Gründe wären, auf Social Freezing zurückzugreifen. ${ }^{11}$ Die Ergebnisse sind in der Abbildung 10 abgebildet.

11 Es handelt sich um eine Frage, die nur denjenigen gestellt wurde, die, bevor sie Detailinformationen zu Verfahren, Risiken, Erfolgsaussichten, Kosten und Rechtslage erhielten, angegeben hatten, dass sie sich vorstellen könnten, ihre Eizellen vorsorglich einfrieren zu lassen, oder dies bereits gemacht haben $(n=217)$. 
Abbildung 10: Wichtigster Beweggrund für Social Freezing nach Alterskategorie, in Prozent

Weil ich befürchte, dass meine Fruchtbarkeit eingeschränkt ist

Damit ich mehr Zeit habe, mich für ein Kind bereit zu fühlen

Damit ich mehr Zeit für die Partnersuche habe

Damit ich meine Karriere vorantreiben kann, bevor ich Kinder habe

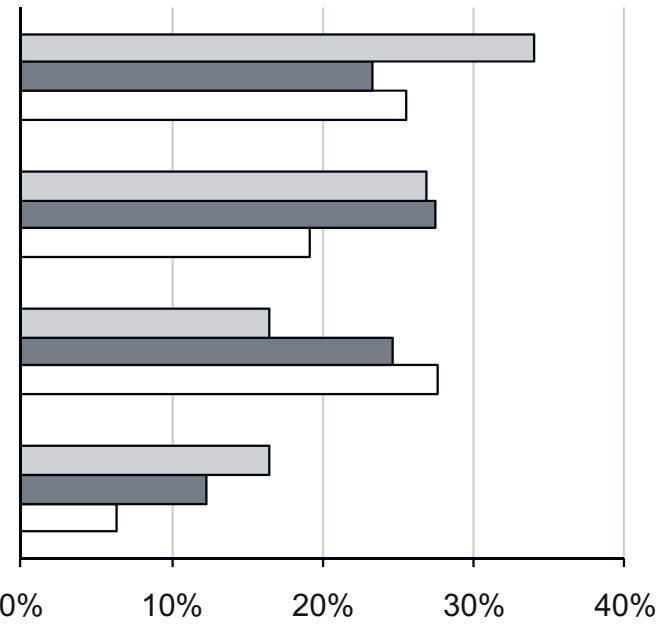

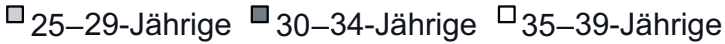

Quelle: Befragung Social Freezing 2018; $\mathrm{n}=207$.

An erster Stelle wurde die Befürchtung einer eingeschränkten Fruchtbarkeit genannt $(29 \%)$, dicht gefolgt von dem Wunsch, mehr Zeit zu haben, um sich für ein Kind bereit zu fühlen (25\%). Auch der Wunsch nach mehr Zeit für die Partnersuche wurde häufig genannt (22\%). Dies entspricht den Ergebnissen aus qualitativen Studien zu den Motivationen von Frauen, die Social Freezing in Anspruch nehmen. Am seltensten (13\%) wurde die Karriereplanung als Grund angegeben, obwohl diese Motivation in der Literatur und in den Medien sehr häufig diskutiert wird. Elf Prozent der befragten Frauen äusserten sich nicht zu dieser Frage.

Die vertiefte Auswertung nach soziodemografischen Angaben zeigt, dass in allen Altersgruppen die Befürchtung, die Fruchtbarkeit könnte eingeschränkt sein, eine wichtige Rolle spielt. Sie wird in der Altersgruppe der 25- bis 29-Jährigen am häufigsten als Grund genannt und in derjenigen der 35- bis 39-Jährigen am zweithäufigsten. In der Gruppe der 30- bis 34-Jährigen wird sie mit 23,3 Prozent fast ebenso häufig genannt wie die Partnersuche mit 24,7 Pro- 
zent. Als häufigster Beweggrund für Social Freezing wird in der Altersgruppe der 35- bis 39-Jährigen die Zeit für die Partnersuche genannt, in der Altersgruppe der 30- bis 34-Jährigen die Zeit, sich für ein Kind bereit zu fühlen. Über alle Altersgruppen hinweg am wenigsten angegeben wurde der Grund, die Karriere vorantreiben zu wollen. Am häufigsten wird dieser Grund noch von den 25bis 29-Jährigen angegeben, und zwar mit 17 Prozent gleich häufig wie die Partnersuche; bei den 30- bis 34-Jährigen sind es 12 Prozent und bei den 35- bis 39-Jährigen 6 Prozent. Die Unterschiede zwischen Alterskategorie und Beweggründen sind jedoch nicht signifikant.

Frauen, die aufgrund eines Wunsches nach mehr Zeit für ein Kind auf Social Freezing zurückgreifen, haben im Durchschnitt eine eher tiefere Bildung als Frauen, die aus einem der anderen Gründe auf Social Freezing zurückgreifen. Frauen, die auf Social Freezing zurückgreifen würden, um ihre Karriere voranzutreiben, haben hingegen im Durchschnitt den höchsten Bildungsabschluss. Diese Ergebnisse sind jedoch, wie auch diejenigen zwischen den Sprachregionen (Deutschschweiz und französische Schweiz), nicht signifikant.

\subsubsection{Alter für die Einfrierung der Eizellen}

Frauen, die sich eine vorsorgliche Einfrierung der Eizellen vorstellen könnten beziehungweise die dies bereits gemacht haben, wurden gefragt, in welchem Alter sie dies spätestens tun würden (vgl. Abbildung 11). Jeweils 36 Prozent der befragten Frauen würden ihre Eizellen zwischen 30 und 34 beziehungsweise zwischen 35 und 39 einfrieren lassen. Insgesamt 73 Prozent würden somit ihre Eizellen zwischen 30 und 39 Jahren einfrieren lassen. Nur 11 Prozent ziehen ein Alter von 25 bis 29 für das Einfrieren ihrer Eizellen in Betracht und gerade mal 2 Prozent ( 5 Personen) ein Alter von unter 25 Jahren. Immerhin 5 Prozent (12 Personen) könnten sich vorstellen, im Alter von über 40 Jahren ihre Eizellen einfrieren zu lassen. Diese Ergebnisse spiegeln die Ergebnisse aus Studien in anderen Ländern und die Erfahrungen der hiesigen Reproduktionsmedizinerinnen und -mediziner. Sie machen deutlich, dass viele Frauen die Möglichkeiten der reproduktionsmedizinischen Verfahren und des Social Freezing im Speziellen überschätzen. In der Altersspanne 30 bis 35 bietet sich Social Freezing aus individuellen Kosten-Nutzen-Überlegungen am ehesten an, weil dann die Er- 
folgsaussichten noch relativ gut sind und die Chancen, dass die Frauen auf die Eizellen zurückgreifen müssen, voraussehbar sind.

\section{Abbildung 11: $\quad$ Alter für die Einfrierung der Eizellen}

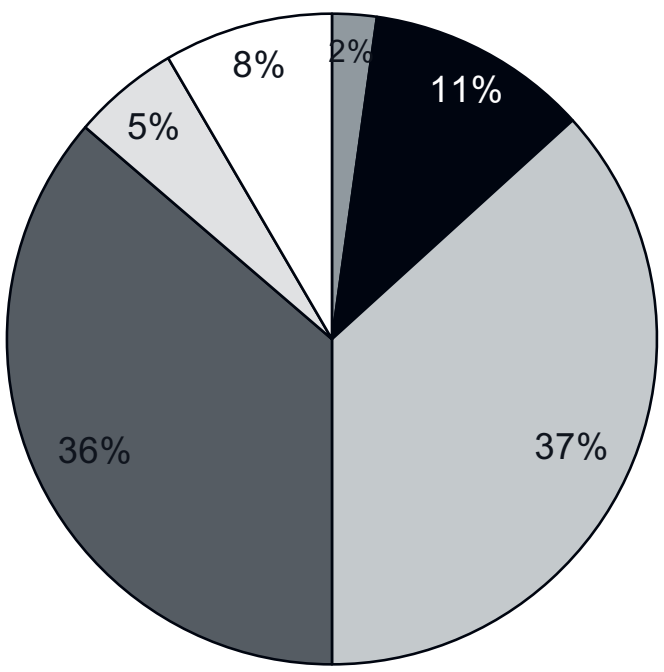

口Unter 25 Jahren

$\square$ Zwischen 30 und 34 Jahren 口Über 40 Jahren
- Zwischen 25 und 29 Jahren

$\square$ Zwischen 35 und 39 Jahren

$\square$ Weiss nicht/keine Antwort

Quelle: Befragung Social Freezing 2018; $n=226$.

\subsection{5}

\section{Höchstalter für Mutterschaft}

Allen Frauen wurde in der Befragung zudem die Frage gestellt, in welchem Alter sie spätestens Mutter werden wollten. Zwei Drittel der Frauen möchten unter 40 Jahren Mutter werden (65\%). Zwischen 40 und 44 Jahren sind es noch 13 Prozent. Die Anzahl der Frauen, die zwischen 45 und 49 beziehungsweise 50 und 54 Jahren Mutter werden möchten, ist mit drei und zwei Frauen verschwindend gering. Ab 55 möchte niemand mehr Mutter werden. 18 Prozent geben an, nicht Mutter werden zu wollen oder zu können. Die Befürchtungen, 
dass die Anzahl hochaltriger Mütter zunehmen wird, werden durch diese Umfrage also nicht bestätigt.

Der Durchschnitt des gewünschten Höchstalters für Mutterschaft ist bei SingleFrauen und den befragten Frauen aus der höchsten Alterskategorie (35- bis 39jährig) signifikant am höchsten. In Bezug auf Bildung lassen sich keine signifikanten Unterschiede bei dem gewünschten Höchstalter für Mutterschaft feststellen, dies trifft auch auf die unterschiedlichen Landesteile zu.

\subsubsection{Potenzial des Social Freezing}

Gestützt auf die Ergebnisse dieser Befragung wird das Potenzial an Frauen eingeschätzt, die künftig ihre Eizellen werden entnehmen und vorsorglich einfrieren lassen. Es werden drei Szenarien angegeben:

- Das hohe Szenario beruht auf den 34 Prozent der Befragten, die sich grundsätzlich auch nach Erhalt von Detailinformationen vorstellen könnten, ihre Eizellen vorsorglich einfrieren zu lassen. In der Realität dürften wesentlich weniger Befragte ein Social Freezing konkret in Erwägung ziehen, weil sich ihre Lebensumstände ändern oder sie noch detailliertere Informationen zu den Risiken davon abhalten würden. Zudem bedeutet die Absicht, etwas zu tun, in der Realität nicht, dass sie dann auch immer umgesetzt wird.

- Das tiefe Szenario umfasst grundsätzlich das eine Prozent der Befragten, welche Social Freezing bereits in Anspruch genommen haben. Dieser Anteil dürfte wiederum für ein künftiges Potenzial zu tief sein, da die Kenntnis in Bezug auf Social Freezing in der Schweiz im Vergleich zu ausländischen Studien mit 48 Prozent noch eher gering ist und davon auszugehen ist, dass das Interesse am Social Freezing mit zunehmender Bekanntheit auch noch ansteigen wird.

- Ein mittleres Szenario geht von jenen 34 Prozent der Befragten aus, die sich grundsätzlich vorstellen könnten, ihre Eizellen vorsorglich einfrieren zu lassen. Von diesem Anteil werden diejenigen abgezogen, die bei mindestens einer der Detailinformationen angegeben haben, dass die- 
se ihren Entscheid, Social Freezing in Anspruch zu nehmen, erschweren würde. Damit verbleiben noch knapp 7 Prozent kinderlose Frauen zwischen 25 und 39, die sich vorstellen können, Social Freezing zu beanspruchen, und von keiner Detailinformation negativ in ihrem Entscheid beeinflusst werden. Diese 7 Prozent entsprechen auch in etwa den Ergebnissen aus internationalen Studien und beschreiben den Anteil an Frauen, bei denen sich nicht nur ein grundsätzliches, sondern ein konkretes Interesse am Social Freezing manifestiert.

Gemäss Daten des Bundesamtes für Statistik gibt es in der Schweiz rund 440'000 kinderlose Frauen zwischen 25 und 39 Jahren (BFS 2013/2016). Gemäss hohem Szenario würde demnach das potenzielle Interesse bei rund 150'000 Frauen liegen, die im Zeitraum zwischen 25 und 39 Jahren ihre Eizellen einmal einfrieren lassen würden. Teilt man diese Anzahl durch den Beobachtungszeitraum von 15 Jahren, ${ }^{12}$ so ergibt dies grob geschätzt eine Anzahl von knapp 10'000 Frauen pro Jahr, die ihre Eizellen einfrieren lassen würden. Gemäss mittlerem Szenario liegt das Potenzial bei 30'800 Frauen über den gesamten Zeitraum betrachtet, was einem jährlichen Potenzial von rund 2000 Frauen entspricht. Beim tiefem Szenario sind es 4400 Frauen, die im Zeitraum zwischen 25 und 39 Jahren ihre Eizellen einfrieren lassen würden. Das jährliche Potenzial würde knapp 300 Frauen betragen.

Wie viele Kinder letztlich jährlich aufgrund dieser Szenarien mittels Social Freezing geboren werden, kann nur sehr grob eingeschätzt werden. Vom Einfrieren der Eizellen bis zur Geburt eines Kindes ist es ein langer Weg. In der nachfolgenden Tabelle wird dieser Weg für die drei Szenarien aufgezeigt. Die Schätzungen basieren auf folgenden zwei Annahmen, welche auf internationalen Studien und Einschätzungen von Expertinnen und Experten beruhen:

- 20 Prozent der Frauen, die ihre Eizellen eingefroren haben, lassen diese später in vitro befruchten.

- 50 Prozent der Frauen, die ihre Eizelle in vitro befruchtet haben, bringen ein Kind zur Welt. 
Ausgehend vom mittleren Szenario würden demnach einst rund 200 Kinder pro Jahr mittels Social Freezing zur Welt kommen.

Tabelle 4: $\quad$ Szenarien zur potenziellen Entwicklung der Nachfrage nach Social Freezing

\begin{tabular}{|c|c|c|c|c|}
\hline & $\begin{array}{l}\text { Eizellen } \\
\text { einfrieren } \\
\text { Grund- } \\
\text { gesamtheit: } \\
440 \text { '000 kinder- } \\
\text { lose Frauen im } \\
\text { Alter von } 25 \text { bis } \\
39 \text { Jahren }\end{array}$ & $\begin{array}{l}\text { Eizellen } \\
\text { einfrieren }\end{array}$ & $\begin{array}{l}\text { Befruchtung } \\
\text { in vitro der } \\
\text { einge- } \\
\text { frorenen } \\
\text { Eizellen* }\end{array}$ & $\begin{array}{l}\text { Geburt eines } \\
\text { Kindes auf- } \\
\text { grund des } \\
\text { Social } \\
\text { Freezing** }\end{array}$ \\
\hline & $\begin{array}{l}\text { Über Beobach- } \\
\text { tungszeitraum } \\
\text { von } 15 \text { Jahren }\end{array}$ & \multicolumn{3}{|l|}{ Pro Jahr } \\
\hline $\begin{array}{l}\text { Hohes } \\
\text { Szenario: } 34 \%\end{array}$ & $149^{\prime} 600$ & 9973 & 1995 & 997 \\
\hline $\begin{array}{l}\text { Mittleres } \\
\text { Szenario: } 7 \%\end{array}$ & $30 ’ 800$ & 2053 & 411 & 205 \\
\hline $\begin{array}{l}\text { Tiefes- } \\
\text { szenario: } 1 \%\end{array}$ & 4400 & 293 & 59 & 29 \\
\hline
\end{tabular}

Quelle: Berechnungen auf der Grundlage der Befragung Social Freezing 2018 und der Daten des BFS 2013/2016. * Annahme: $20 \%$ der Frauen, die ihre Eizellen eingefroren haben, lassen diese in vitro befruchten. ${ }^{* *}$ Annahme: $50 \%$ der Frauen, die ihre Eizelle in vitro befruchtet haben, bringen ein Kind zur Welt. 


\section{Diskussion des Social Freezing in der internationalen Literatur}

In diesem Kapitel werden die ethische (vgl. Kapitel 5.1), die soziale (vgl. 5.2) und die finanzielle (vgl. Kapitel 5.3) Dimension des Social Freezing diskutiert. Die Diskussion dieser Dimensionen in der wissenschaftlichen Literatur wird stark durch Ethikerinnen und Ethiker geprägt (Bernstein und Wiesemann 2014, Cattapan et al. 2014, Cutas und Smajdor 2015, Dondorp und De Wert 2009, Goold und Savulescu 2009, Harwood 2015, Hens 2017, Kovács 2013, Kress 2013, Mertes 2013 und 2017, Mertes und Pennings 2012, NEK 2017, Pennings 2013, Roth 2016, Shkedi-Rafid und Hashiloni-Dolev 2012). Weiter werden die Debatten durch (reproduktions-)medizinische Fachgesellschaften (ASRM 2012, ESHRE 2012, Académie nationale de médecine 2017) sowie Ärztinnen und Ärzte (Belaisch-Allart et al. 2013, Lockwood 2011, Mechanick 2017, Wunder 2014) bestimmt. Wichtige Beiträge liefern auch Sozialwissenschaftler und Sozialwissenschaftlerinnen (Baldwin 2016, Waldby 2014).

\subsection{Diskussion der ethischen Dimension des Social Freezing}

Die ethische Dimension des Social Freezing wird durch Debatten bezüglich Fortpflanzungsfreiheit, Kindeswohl und Verwendung der überzähligen Eizellen geprägt.

\subsubsection{Fortpflanzungsfreiheit}

Ein wichtiges in Zusammenhang mit Social Freezing diskutiertes ethisches Prinzip betrifft die Fortpflanzungsfreiheit respektive die reproduktive Autonomie. Die Fortpflanzungsfreiheit umfasst den freien Entscheid, ob, wann und wie jemand sich fortpflanzen und ein Kind bekommen möchte (Wiesemann 2015, 
zitiert in Roth 2016). Dies kann einerseits bedeuten, dass insbesondere der Staat keine Schranken errichtet, welche die Individuen an der Verwirklichung ihres Kinderwunsches hindern (negative Freiheit). Andererseits kann darunter verstanden werden, dass der Staat die notwendigen Voraussetzungen schafft, dass ein Individuum seinen Kinderwunsch erfüllen kann (positive Freiheit) (NEK 2013). Zudem wird die Fortpflanzungsfreiheit in Zusammenhang mit einem liberalen und einem relationalen Autonomiekonzept diskutiert. Vertretende eines liberalen Autonomiekonzepts gehen davon aus, dass die Individuen eigenständig über ihre Fortpflanzung entscheiden können. Vertretende eines relationalen Autonomiekonzepts sind hingegen der Ansicht, dass dieser Entscheid immer in einen bestimmten gesellschaftlichen Kontext eingebettet ist und von diesem mitbestimmt wird (Van der Ven 2017).

Befürwortende des Social Freezing berufen sich auf ein liberales Autonomiekonzept, in dem die individuelle Freiheit im Zentrum steht. Nur die Betroffenen können über die Nutzung dieser Technologie entscheiden, weil sie ihre Lebensumstände und Gefühle besser kennen als Aussenstehende (Van der Ven 2017). Verfechtende eines liberalen Autonomieverständnisses lehnen Einschränkungen im Zugang zum Social Freezing zwecks Schutz der Frauen vor falschen Versprechungen durch die Reproduktionsmedizin als paternalistisch ab (ESHR 2012, Mertes 2011, Académie nationale de médecine 2017). Sie argumentieren, dass die Frauen die Erfolgsaussichten des Verfahrens durchaus beurteilen können (Bernstein und Wiesemann 2014), dass sie sich mit Social Freezing nur eine Option unter anderen zur Familiengründung offenhalten (Goold und Savulescu 2009) und dass die Frauen vor allem vermeiden möchten, nicht alle Möglichkeiten zur Erfüllung ihres Kinderwunsches ausgeschöpft zu haben (Mertes 2017). Diese Einschätzungen decken sich mit den Ergebnissen in empirischen Studien zu den Erfahrungen von Frauen, die ihre Eizellen kryokonservieren liessen (vgl. Kapitel 3). Statt für eine Einschränkung der Technologie plädieren sie für eine ausführliche Information der Frauen, was als wichtige Voraussetzung für die Ausübung der reproduktiven Autonomie erachtet wird (ASRM 2012, ESHRE 2012, Roth 2016, Goold und Savulescu 2009, Mertes 2013). In Zusammenhang mit Social Freezing ist eine informierte Entscheidung umso wichtiger, als die Datenlage zu den Risiken für das Kind noch limitiert ist und insbesondere bei älteren Frauen falsche Hoffnungen in Bezug auf die Möglichkeiten des Social Freezing geweckt werden könnten. Interessierte Frauen sollen entsprechend informiert werden, dass Social Freezing keine Garantie für ein Kind bietet und dass eine natürliche Zeugung in jungen Jahren 
Erfolg versprechender ist. Zudem sollten die Frauen Informationen über ihre persönlichen Erfolgsaussichten und die dafür notwendige Anzahl Eizellen erhalten (ESHR 2012, Harwood 2015). In der Schweiz spricht sich auch die Nationale Ethikkommission im Bereich der Humanmedizin (NEK) in ihrer Stellungnahme zum Social Freezing (2017) für eine umfassende Information der Frauen aus.

Dieses liberale Autonomieverständnis berücksichtigt aus Sicht der Kritikerinnen und Kritiker des Social Freezing jedoch ungenügend, dass sich individuelle Präferenzen in bestimmten sozialen Kontexten ausbilden und durch diese geprägt werden. Die Kritikerinnen und Kritiker berufen sich aus diesem Grund auf ein relationales Autonomiekonzept (Van der Ven 2017). Die Inanspruchnahme des Social Freezing wird nicht als Ausdruck eines rationalen und autonomen Entscheides, sondern als Folge gesellschaftlicher Bedingungen wie der mangelnden Vereinbarkeit von Beruf und Familie (vgl. Kapitel 5.2.1) gesehen. Ebenso wird der Wunsch, sich mehrere Optionen für die Familienplanung offenzuhalten und alles Machbare gegen eine abnehmende Infertilität zu unternehmen, nicht als Ausdruck von Autonomie, sondern von neoliberalen Wertvorstellungen gesehen. Gemäss dem neoliberalen Wertekanon hat jeder Einzelne für ein erfülltes Leben zu sorgen und Vorkehrungen gegen allfällige Rückschläge - wie einen unerfüllten Kinderwunsch - zu treffen. Dass der Entscheid für Social Freezing durch verschiedene soziale Faktoren beeinflusst wird, wird dabei ausgeklammert (Baldwin 2016). Entsprechend wird selbst eine ausführliche Information der Frauen als ungenügend erachtet, um einen autonomen Entscheid für Social Freezing zu ermöglichen. Statt den Frauen eine technologische Lösung als Antwort auf die vorherrschenden gesellschaftlichen Bedingungen anzubieten, sollen diese Bedingungen geändert werden (Shkedi-Rafid und Hashiloni-Dolev 2012). Verschiedene Autorinnen und Autoren, darunter die Nationale Ethikkommission im Bereich Humanmedizin (2017), befürworten die Zulassung der Technologie und setzen sich gleichzeitig für eine Veränderung der gesellschaftlichen Bedingungen ein (vgl. Kapitel 5.2.1).

Eine Einschränkung der Fortpflanzungsfreiheit ist nur zulässig, wenn die Fortpflanzungsfreiheit mit dem Kindeswohl (vgl. Kapitel 5.1.2) und gesamtgesellschaftlichen Interessen in Konflikt steht. Die gesellschaftsethische Diskussion fokussiert sich vor allem auf die Chancengleichheit der Geschlechter insbesondere in Zusammenhang mit der Vereinbarkeit von Beruf und Familie (vgl. Kapitel 5.2.1). Andere gesellschaftliche Interessen wie die Auswirkungen einer brei- 
ten Anwendung des Social Freezing auf die öffentliche Gesundheit waren nicht Gegenstand der Diskussion in der internationalen Literatur oder in den Schweizer Medien.

\subsubsection{Kindeswohl}

Sowohl Vertretende eines liberalen als auch eines relationalen Autonomieverständnisses erachten Einschränkungen der Fortpflanzungsfreiheit als zulässig, wenn Dritte von der Entscheidung betroffen sind (Harwood 2015, Roth 2016). In diesem Zusammenhang stellt sich die Frage, inwiefern die Fortpflanzungsfreiheit mit dem Kindeswohl zu vereinbaren ist. Der Begriff des Kindeswohls ist allerdings weder in ethischer, juristischer noch sozialwissenschaftlicher Hinsicht genau definiert (NEK 2013, Simoni 2012). In den Diskussionen zum Social Freezing wird der Begriff des Kindeswohls nicht näher definiert, aber auf die Risiken der Technologie für die Kinder verwiesen. Diese können einerseits durch die Technologie an und für sich als auch durch eine Schwangerschaft in fortgeschrittenem Alter bedingt sein. In diesem Zusammenhang werden vor allem die Risiken einer späten Schwangerschaft, einer späten Mutterschaft sowie einer frühen Verwaisung diskutiert. Es stellt sich zudem die Frage, ob diese Risiken eine Altersgrenze für die Befruchtung der Eizellen in vitro rechtfertigen.

\section{Einfluss der Technologie auf das Kindeswohl}

Die Auswirkungen der Technologie werden selten diskutiert. Allfällige gesundheitliche Risiken für das Kind aufgrund epigenetischer Veränderungen in Zusammenhang mit ovarieller Stimulation und Befruchtung der Eizellen in vitro sind nicht Gegenstand der Diskussion. Im Fokus der Diskussion stehen mögliche Auswirkungen der Kryokonservierung der Eizellen und es wird auf die beruhigende, wenn auch dünne Datenlage verwiesen. Es fehlen zudem Langzeitdaten zur gesundheitlichen, psychischen und sozialen Entwicklung der Kinder der Kryokonservierung (vgl. Kapitel 2.2.3). Eine zurückhaltende Anwendung der Kryokonservierung empfiehlt sich entsprechend gestützt auf das Vorsorgeprinzip (Linkeviciute et al. 2015). Dieses Prinzip sieht vor, dass Risiken für die 
menschliche Gesundheit auch auf unvollständiger Wissensbasis vermieden oder verringert werden sollen.

Goold und Savulescu (2009) sprechen sich indes mit Verweis auf das Nichtidentitätsproblem gegen eine Einschränkung des Social Freezing aus. Selbst wenn Social Freezing zu einer gesundheitlichen Beeinträchtigung führen würde, hätte das betroffene Kind kein Interesse am Verbot dieser Technologie. Ohne diese Technologie wäre das Kind entweder nicht geboren oder es wäre ein anderes Kind mit einer alternativen Zeugungsmethode zu einem anderen Zeitpunkt gezeugt worden. Eine Einschränkung des Zugangs zum Social Freezing wäre nur dann gerechtfertigt, wenn das Leben für das Kind nicht lebenswert wäre. Weiter ist für Goold und Savulescu unverständlich, dass die Beurteilung dieser Risiken bei Frauen, die diese Technologien aus sozialen Gründen in Anspruch nehmen, anders ausfällt als bei anderen Patientinnen. Wenn die Konservierung und Befruchtung der Eizellen in vitro effektiv mit einem beträchtlichen Risiko für das Kind verbunden wären, dürften sie auch Krebspatientinnen und unfruchtbaren Paaren nicht angeboten werden. Diese Argumentation blendet allerdings aus, dass bei einer allgemeinen Einschränkung der Fortpflanzungsfreiheit durchaus Ausnahmen für besonders benachteiligte Paare möglich sind.

In gleichem Sinne wird darauf hingewiesen, dass bezüglich der Auswirkungen fortpflanzungsmedizinischer Technologien auf das Kindeswohl bei Frauen andere Massstäbe angewendet werden als bei Männern. So steht die Befruchtung mittels intrazytoplasmatischer Spermieninjektion (ICSI) im Verdacht, Fehlbildungen und epigenetische Veränderungen beim Nachwuchs zu erzeugen. Im Gegensatz zur Eizellkonservierung wurde diese Technologie zur Behandlung männlicher Fruchtbarkeit viel schneller anerkannt und nie einem experimentellen Status unterstellt (Rybak et al. 2009). Ebenso wird es als diskriminierend erachtet, dass im Gegensatz zur Eizellkonservierung bei der Spermienkonservierung keine Unterscheidung zwischen medizinischen und sozialen Gründen vorgenommen wird. So werden beispielsweise Spermien von Männern eingefroren, die in einem giftigen Umfeld arbeiten oder riskante Sportarten (Hockey, Football, Radfahren) ausüben. Auch vor einer Vasektomie lassen Männer teilweise Spermien einfrieren, um ihre Fertilität für eine künftige Beziehung zu erhalten (ESHR 2012, Dondorp und De Wert 2009, Goold und Savulescu 2009, Académie nationale de médecine 2017, Belaisch-Allart et al. 2013). 


\section{Einfluss einer Schwangerschaft in fortgeschrittenem Alter auf das Kindeswohl}

Die medizinischen Risiken einer späten Schwangerschaft für Mutter und Kind sind relativ unbestritten (vgl. Kapitel 2.2.3). Es gibt jedoch auch Autoren und Autorinnen, die diese Risiken nuancieren. So wird argumentiert, dass es wenige Daten zu den Schwangerschaftsrisiken bei Frauen über 50 gibt und dass die wenigen Daten widersprüchlich sind (Dondorp und De Wert 2009). Zudem werden in Verbindung mit späten Schwangerschaften selten schützende Faktoren erwähnt. Frauen, die ihre Eizellen einfrieren lassen, verfügen in der Regel über einen hohen sozioökonomischen Status. Ein solcher korreliert mit einer signifikanten Reduktion von Frühgeburten bei Schwangerschaften in fortgeschrittenem Alter (Bernstein und Wiesemann 2014). Umgekehrt können junge Frauen individuell aufgrund von Vorerkrankungen ein hohes Risikoprofil für eine Schwangerschaft aufweisen. Ein allfälliges Risiko für ihr Kind wird aber nicht als legitimer Grund gesehen, um ihr Fortpflanzungsrecht einzuschränken. Stattdessen wird bei einer Risikoschwangerschaft die Bedeutung einer umfassenden Beratung und eines entsprechend informierten Entscheides unterstrichen (Roth 2016).

Weiter wird argumentiert, dass den Risiken einer späten Vaterschaft sowohl in der öffentlichen als auch in der wissenschaftlichen Diskussion weniger Bedeutung beigemessen wird als dem mütterlichen Alter (Hens 2017, Bernstein und Wiesemann 2014). So zeigte eine Medienanalyse in den USA, dass die mütterlichen Risiken stärker hervorgehoben werden und die Artikel in einem anklagenden Ton verfasst sind. Der Tonfall in der Berichterstattung zu den Risiken einer späten Vaterschaft war hingegen beruhigend (Lisa Campo-Engelstein et al. 2015, zitiert in Hens 2017). Eine Suche in PubMed, einer Datenbank mit medizinischen Publikationen, im Jahr 2014 ergab 109 Treffer für das Schlüsselwort "späte Mutterschaft» im Vergleich zu 17 Treffern für "späte Vaterschaft» (Bernstein und Wiesemann 2014). Verschiedene Studien zeigen indes, dass sich ein erhöhtes väterliches Alter negativ auf die Gesundheit des Nachwuchses auswirkt. Eine Vaterschaft in fortgeschrittenem Alter wird mit einem erhöhten Risiko für Fehlgeburten (Woodward und MacDuffie 2016, Sobotka 2009), Fehlbildungen der Lippen- und Gaumenspalte (Hens 2016) sowie Autismus, Schizophrenie und bipolaren Störungen in Verbindung gebracht (Woodward und MacDuffie 2016, Hens 2017, Sobotka 2009, Mechanick 2017, Simoni 2012). Als Erklärung werden Probleme bei der Zellteilung in den primordialen männlichen Keimzellen herangezogen. Während bei 30-jährigen 
Männern 380 solcher Zellteilungen erfolgt sind, sind es bei 50-jährigen Männern bereits 840 . Je mehr solcher Zellteilungen erfolgt sind, desto höher ist das Risiko für Neumutationen (novo mutations) in den Genen (Woodward und MacDuffie 2016).

\section{Einfluss einer Mutterschaft in fortgeschrittenem Alter auf das Kindeswohl}

Die psychologischen und sozialen Voraussetzungen einer späten Mutterschaft werden rege diskutiert. Als Nachteil einer solchen Mutterschaft werden vor allem die nachlassenden körperlichen und psychischen Kräfte hervorgehoben. Es wird befürchtet, dass ältere Mütter stärker unter Schlafmangel leiden und weniger Energie für die anstrengende Versorgung von Kleinkindern haben als jüngere Mütter (Shkedi-Rafid und Hashiloni-Dolev 2012, Datta 2017). In einer Studie mit 117 Personen, die dank In-vitro-Befruchtung im Alter von über 40 Jahren Eltern wurden, beklagen sich 40 Prozent der Mütter und ein Viertel der Väter über Energiemangel infolge der Elternschaft (MacDougall, Beyene, Nachtigall 2012, zitiert in Woodward und MacDuffie 2016). Zudem sind ältere Mütter möglicherweise gebrechlicher und können darum weniger gut mit ihren Kindern spielen (Roth 2016). Schliesslich wird infrage gestellt, dass Mütter Mitte 60 die notwendigen emotionalen und körperlichen Voraussetzungen mitbringen, um mit pubertierenden Kindern umzugehen (Wunder 2014, Goold und Savulescu 2009).

Einzelne Autorinnen und Autoren bestreiten hingegen diese Nachteile einer Mutterschaft in fortgeschrittenem Alter. Goold und Savulescu (2009) zitieren eine Studie, welche den Stresslevel und die physische Funktionsfähigkeit bei 150 Frauen in den Altersgruppen von 30, 40 und 50 Jahren untersuchte und keine signifikanten Unterschiede zwischen den Altersgruppen feststellen konnte. Cutas und Smajdor (2015) argumentieren, dass Grosseltern eine wichtige Rolle bei der Kindererziehung spielen und dafür nicht als zu alt beurteilt werden. Zudem würden sich 60-jährige Frauen auch in der Pflege ihrer betagten Eltern engagieren, was sie als anspruchsvoller erachten als die Betreuung eines Kindes.

Daneben werden verschiedene Vorteile einer Mutterschaft in fortgeschrittenem Alter hervorgehoben, die auch von Frauen mit kryokonservierten Eizellen betont wurden (vgl. Kapitel 3.1.3). Dazu zählt einerseits, dass die Kinder älterer Mütter 
in besseren sozioökonomischen Verhältnissen aufwachsen als Kinder von jüngeren Müttern. Die Familien verfügen über finanzielle Sicherheit und die Elternbeziehung ist stabiler (ESHR 2012, Académie nationale de médecine 2017, Wunder 2014, Linkeviciute et al. 2015, Roth 2016, Bernstein und Wiesemann 2014). Andererseits sind auch die psychologischen Voraussetzungen für eine erfolgreiche Mutterschaft gegeben. Dies führt zu einer besseren Mutter-KindBeziehung (ESHR 2012, Wunder 2014, Barnes et al. 2014, Camberis et al. 2016). Kinder von älteren Müttern weisen eine bessere sprachliche Entwicklung auf und sind weniger von Unfällen sowie Krankenhauseinweisungen betroffen (Roth 2016, Wunder 2014). Ihre Schulleistungen sind besser und sie leiden weniger unter Suchterkrankungen (Sobotka 2009). Allerdings sind die entsprechenden Studien schwer interpretierbar, weil ein hochkomplexes Netzwerk von Einflussfaktoren besteht.

Schliesslich wird betont, dass auch jüngere Mütter von Gebrechlichkeit betroffen sein können, die sie in der Kinderbetreuung einschränkt (Cutas und Smajdor 2015). Zudem stellen sich entsprechende Fragen auch bei alten Vätern, die in der Öffentlichkeit jedoch in der Regel positiv beurteilt werden (Mechanick 2017).

\section{Risiko einer frühen Verwaisung}

In Zusammenhang mit Social Freezing wird ebenfalls das aktive Herbeiführen einer Situation kritisiert, in der ein Verwaisungsrisiko in frühem Kindesalter besteht (Harwood 2015, Roth 2016, Sobotka 2009, Wunder 2014). Die Wahrscheinlichkeit, dass eine 50-jährige Mutter am 20. Geburtstag ihres Kindes bereits verstorben ist, beträgt 15 Prozent. Bei einer 55-Jährigen ist die Wahrscheinlichkeit, dass sie bereits vor der Pubertät ihrer Kinder verstirbt, im Vergleich zu einer 35-Jährigen fünfmal höher. Die Verwaisung im Kindesalter kann zu sozialen Problemen wie den Rückzug von Gleichaltrigen oder zu mangelnder Sozialkompetenz führen (Woodward und MacDuffie 2016). Auch psychologische Probleme wie Depressionen, Selbstverletzungen und Suizide werden in Verbindung mit dem frühen Tod eines Elternteils in Verbindung gebracht (Mechanick 2017, Woodward und MacDuffie 2016). Die Traumatisierung infolge eines Verlustes der Eltern vor dem 35. Lebensjahr wird zudem als eine mögliche Erklärung für eine reduzierte Lebenserwartung von Kindern mit älteren Eltern in Betracht gezogen (Woodward und MacDuffie 2016). 
Das Verwaisungsrisiko genügt einzelnen Autoren jedoch nicht, die Nutzung des Social Freezing zu limitieren. Sie argumentieren, dass auch der Nachwuchs von jüngeren Müttern ein erhöhtes Verwaisungsrisiko aufweisen kann. So bestehen beispielsweise keine Vorbehalte gegen die Eizellkonservierung bei Krebspatientinnen, obwohl bei diesen die Erkrankung erneut ausbrechen kann und sie möglicherweise eine reduzierte Lebenserwartung haben (Cutas und Smajdor 2015). Weiter wird angemerkt, dass es kaum Vorbehalte gegen eine Vaterschaft in hohem Alter gibt, wenn die Partnerin jünger ist und die Kindererziehung bis zur Volljährigkeit sicherstellen kann. Bei Frauen mit jüngeren Partnern sollten die gleichen Spielregeln gelten (Bernstein und Wiesemann 2014, ESHR 2012). Der Verlust eines Elternteils scheint jedoch so gravierend, dass auch die Präsenz des zweiten Elternteils keinen ausreichend hohen Schutzfaktor darstellt (Mechanick 2017).

\section{Notwendigkeit einer Alterslimite für die Nutzung der eingefrorenen Eizellen}

Ob eine Alterslimite für die Nutzung der eingefrorenen Eizellen notwendig ist, hängt sehr stark davon ab, in welchem Alter die Mütter darauf zurückgreifen. Wenn sie lediglich «einige Extrajahre kaufen» und die Eizellen bis Mitte 40 nutzen (ESHR 2012, Linkeviciute et al. 2015, Dondorp und De Wert 2009), sind die Schwangerschaftsrisiken noch relativ gering. Die Mütter dürften ihre Kinder bis ins Erwachsenenalter begleiten können. Alternativ könnte der Gesetzgeber mit einer Alterslimite dafür sorgen, dass das Kindeswohl nicht beeinträchtigt ist (Harwood 2015, Roth 2016, Mechanick 2017). Befürworterinnen und Befürworter einer Alterslimite argumentieren, dass es sich bei der Fortpflanzungsfreiheit um ein negatives und nicht um ein positives Recht handelt. Der Staat darf die Fortpflanzung zwar nicht einschränken, er ist aber auch nicht verpflichtet, den Zugang zu reproduktionsmedizinischen Verfahren in jedem Alter sicherzustellen (Mechanick 2017). Um Schwangerschaftsrisiken zu reduzieren, dürfte eine Alterslimite Mitte 40 sinnvoll sein (vgl. Kapitel 2.2). Bei einer bei guter Gesundheit durchschnittlichen Lebenserwartung von 75 Jahren dürfte eine Mutterschaft bis zum 50. Lebensjahr das Risiko einer Verwaisung vor der Volljährigkeit reduzieren (Belaisch-Allart et al. 2013). Dabei wird es als wichtig erachtet, dass sich die Beurteilung der gesundheitlichen Risiken für den Nachwuchs nicht auf das mütterliche Alter beschränkt, sondern auch Risiken in Zusammenhang mit einem fortgeschrittenen männlichen Alter miteinbezieht (Hens 2017, Bernstein und Wiesemann 2014). Wenn ab einem bestimmten Alter eine gute Kinder- 
erziehung nicht mehr möglich ist, sollte diese Altersgrenze sowohl für Frauen als auch Männer gelten (Goold und Savulescu 2009). Die Nationale Ethikkommission (2017) spricht sich in ihrer Stellungnahme zum Social Freezing dafür aus, dass die medizinischen Risiken aufgrund von Richtlinien medizinischer Fachgesellschaften beurteilt werden sollen. Der Gesetzgeber könnte lediglich gestützt auf sozialethische Überlegungen eine Alterslimite für die Inanspruchnahme des Social Freezing formulieren.

\subsubsection{Verwendung überzähliger Eizellen}

Bei der Hormonstimulation und der Eizellentnahme besteht das ethische Dilemma, ob Eizellen entnommen werden dürfen, wenn diese später nicht sicher genutzt werden, weil die Frauen beispielsweise auf natürlichem Weg schwanger werden. Im Gegensatz zu Embryonen ist die Vernichtung überzähliger Eizellen weniger problematisch, da Eizellen sowohl rechtlich als auch ethisch nicht als ein schützenwertes Gut angesehen werden (Kress 2013). Die European Society of Human Reproduction and Embryology (ESHRE) empfiehlt, vor der Eizellentnahme verschiedene Verwendungszwecke für überfällige Eizellen zu diskutieren. Neben der Zerstörung der Eizellen können diese auch der Wissenschaft zur Verfügung gestellt oder unfruchtbaren Frauen gespendet werden. Bei einer Spende zur Behandlung von Unfruchtbarkeit respektive zu Forschungszwecken müssten allerdings die Eigentumsrechte an den kryokonservierten Eizellen geregelt werden. Verschiedene Szenarien zur Übertragung von Eigentumsrechten sind denkbar. So könnte eine Frau ihre kryokonservierten Eizellen direkt an eine andere ausgewählte Frau respektive Forschungsinstitution spenden. Sie könnte die Eigentumsrechte an ihren kryokonservierten Eizellen auch an die Eizellbank abtreten, die sich um die Suche nach Abnehmern der Eizellen kümmert. Falls diese Abnehmer die Eigentumsrechte an den Eizellen von der Eizellbank erwerben und nicht alle Eizellen verwenden, könnten sie die erworbenen Eizellen weiterverkaufen (Robertson 2014).

\section{Social Freezing in Kombination mit Eizellspende}

Die Eizellspende ist zurzeit in der Schweiz noch nicht erlaubt. Gemäss einer parlamentarischen Initiative sowie einer Stellungnahme der Nationalen Ethik- 
kommission im Bereich der Humanmedizin (2013) sollte dieses Verbot aufgehoben werden, weil es in Anbetracht der Zulässigkeit der Spermienspende als diskriminierend erachtet wird. Gemäss der European Society of Human Reproduction and Embryology (ESHRE 2012) spricht einiges dafür, die Eizellspende und Social Freezing gemeinsam zu diskutieren. So könnte ein Teil der durch Social Freezing gewonnenen Eizellen unfruchtbaren Frauen gespendet werden. Als gewichtiger Vorteil einer solchen Spende wird erachtet, dass sich die Frauen nicht ausschliesslich für eine fremde Eizellempfängerin einer aufwendigen Behandlung unterziehen.

Für die ethische Beurteilung dieser Frage ist es allerdings wichtig, den Zeitpunkt der Eizellspende zu berücksichtigen. Die Eizellen könnten sofort nach der Entnahme unfruchtbaren Frauen gespendet werden. So bieten in Grossbritannien einzelne Kliniken die Möglichkeit von «Freeze and Share», indem sie bei unter 35-jährigen Frauen die Kosten für die Eizellentnahme übernehmen und die Frauen im Gegenzug die Hälfte ihrer Eizellen spenden. In Zusammenhang mit diesen «Freeze and Share»-Arrangements wird kritisiert, dass die Spenderin möglicherweise nie auf ihre eigenen Eizellen zurückgreifen wird, während die Empfängerin dank der gespendeten Eizellen Mutter wird. Diese Situation kann sich für die Spenderin als psychisch belastend erweisen (Mertes et al. 2012). Die Bedenken fallen weniger ins Gewicht, wenn die Frau ihre überzähligen Eizellen erst spendet, nachdem sie Mutter geworden ist oder mit dem Kinderwunsch abgeschlossen hat. Die psychologischen Folgen einer Eizellspende sind für die Spenderin in einer solchen Situation besser abschätzbar.

Es wird jedoch auch befürchtet, dass mit diesen «Freeze and Share»-Arrangements eine Kommerzialisierung der Eizellen einhergeht und diese nicht mehr aus altruistischen Gründen gespendet werden (Mertes et al. 2012, Robertson 2014, Gupta 2006). Die Angst vor einer Kommerzialisierung wird allerdings als nicht ausreichend erachtet, die Fortpflanzungsfreiheit der Frauen einzuschränken. Für Frauen mit geringen finanziellen Mitteln ist «Freeze and Share» die einzige Option, ihren Kinderwunsch weiterleben zu lassen (Mertes et al. 2012). Der Verkauf von Eizellen wird als Ausdruck eines freien Entscheides und der Selbstbestimmung über den eigenen Körper gesehen. Andere kritisieren dagegen, dass die Anbieter fortpflanzungsmedizinischer Verfahren diese Argumente benutzen, um den Verkauf von Eizellen zu rechtfertigen. Ausgeblendet wird dabei, dass dieser Markt erst durch die asymmetrische Beziehung zwischen 
reichen infertilen Frauen und armen fruchtbaren Frauen entstehen kann (Gupta 2006).

Social Freezing als Alternative zur Eizellspende

Als wichtige Rechtfertigung für Social Freezing wird vorgebracht, dass die Technologie eine Alternative zur Eizellspende darstellt (ESHR 2012, Mertes und Pennings 2012, Lindheim et al. 2014, Académie nationale de médecine 2017, Belaisch-Allart et al. 2013, Wunder 2014). Anstelle von Spendereizellen kann dank Social Freezing in fortgeschrittenem Alter auf eigene Eizellen zurückgegriffen werden, wodurch im Gegensatz zur Eizellspende die genetische Verbindung zwischen Mutter und Kind sichergestellt ist und psychosoziale Komplikationen vermieden werden. Wenn vermehrt junge Frauen ihre Eizellen einfrieren lassen, sinkt auch die Nachfrage nach den raren Spendereizellen. Es gibt allerdings auch Stimmen, die befürchten, dass Social Freezing nicht als Alternative zur Eizellspende zu sehen ist, sondern diese im Gegenteil weiter ankurbelt. Die Frauen könnten ihre kryokonservierten Eizellen nicht nur als Versicherung gegen eine künftige Unfruchtbarkeit sehen, sondern als Ware, die sie bei Nichtverwendung verkaufen können, um ihre Ausgaben für die Kryokonservierung wieder hereinzuspielen. Weil insgesamt mehr Eizellen gelagert werden, könnte der Vertrieb effizienter organisiert werden und so könnten die Preise für die Eizellen sinken. Die Eizellen würden nicht mehr durch die Anbieter fortpflanzungsmedizinischer Verfahren gelagert und vertrieben, sondern durch spezialisierte Eizellbanken. Die kryokonservierten Eizellen einer Frau könnten nicht wie bisher lediglich an eine Empfängerin, sondern an mehrere Empfängerinnen verkauft werden, die sich die Kosten teilen könnten (Robertson 2014). Eine solche Entwicklung dürfte allerdings eher in den USA zu sehen sein, die eine Abgeltung für die Eizellspende im Gegensatz zu den europäischen Ländern erlauben. 


\section{Social Freezing in Kombination mit der Eizellspende zu Forschungszwecken}

Social Freezing wird von Mertes et al. (2012) auch als gute Möglichkeit gesehen, die Anzahl Eizellen für Forschungszwecke zu erhöhen. Zurzeit ist es schwierig, Eizellspenderinnen zu rekrutieren, weil eine Entschädigung in vielen Ländern verboten ist. Zudem bestehen ethische Bedenken gegen diese Praxis. Es wird als unzumutbar erachtet, dass Frauen lediglich für Forschungszwecke die aufwendige und mit medizinischen Risiken verbundene Behandlung zur Eizellentnahme auf sich nehmen müssen (Braun 2012). Beim Social Freezing würden sich die Spenderinnen jedoch zwecks Gewinnung einiger Eizellen einer medizinischen Behandlung unterziehen. Die Forschenden könnten im Rahmen von «Freeze and Share»-Arrangements einen Beitrag an die Behandlungskosten leisten und im Gegenzug einen Teil der Eizellen für ihre Forschung erhalten. Die Eizellspende zu Forschungszwecken hat im Vergleich zur Eizellspende für unfruchtbare Frauen Vorteile. Weit mehr Frauen mit kryokonservierten Eizellen können sich vorstellen, ihre überzähligen Eizellen der Forschung zur Verfügung zu stellen, statt sie unfruchtbaren Frauen zu spenden (vgl. Kapitel 3.1.5). Im Falle einer Eizellspende zu Forschungszwecken sind die Frauen nicht mit der Vorstellung konfrontiert, dass irgendwo ein Kind von ihnen aufwächst (Mertes et al. 2012). Allerdings wäre eine Kostenbeteiligung der Forschenden am Social Freezing eine weitere Strategie, das Verbot der Entschädigung von Eizellspenden zu Forschungszwecken zu umgehen. So wird bereits jetzt sowohl in den USA als auch in Spanien das Entschädigungsverbot unterlaufen. In diesen Ländern wird ein Teil der Eizellen, welche für unfruchtbare Frauen gegen eine Entgeltung respektive Entschädigung gespendet wurden, an Forschungsinstitutionen weitergeleitet (Braun 2012). Allerdings gibt es auch Stimmen, die für eine Entschädigung der Eizellspende zu Forschungszwecken plädieren. Die Entwicklung eines regulierten Marktes für Eizellen würde die Entstehung von unregulierten Schwarzmärkten sowie die Ausnützung von Frauen verhindern (Thompson 2007, zitiert in Braun 2012). Noch einen Schritt weiter geht Cathrine Wadby (2008). Eizellspenderinnen sollen als reproduktive Arbeiterinnen und ökonomische Akteurinnen mit einem wertvollen Beitrag an die Wertschöpfungskette der Stammzellenforschung gesehen werden. Neben der Gewährleistung der Sicherheit und eines informierten Entscheids sollten auch die Rechte dieser reproduktiven Arbeiterinnen anerkannt werden, indem sie sich organisieren und ihre Arbeitsbedingungen wie Nachbetreuung und Versicherung aushandeln dürfen. 


\subsection{Diskussion der gesellschaftlichen Dimension des Social Freezing}

In Zusammenhang mit der gesellschaftlichen Dimension des Social Freezing wird vor allem der Einfluss des Social Freezing auf die Vereinbarkeit von Beruf und Familie rege diskutiert. Ebenfalls für viel Diskussionsstoff sorgt die Frage, ob Social Freezing die Medikalisierung sozialer Probleme begünstigt. Schliesslich wird am Rande auch der Einfluss der Technologie auf die Familienbilder diskutiert.

\subsubsection{Vereinbarkeit von Beruf und Familie}

Der Einfluss des Social Freezing auf die Vereinbarkeit von Beruf und Familie und die Chancengleichheit von Männern und Frauen nimmt einen grossen Stellenwert in den Diskussionen zum Social Freezing ein. Befürworterinnen und Befürworter des Social Freezing sehen diese Technologie als eine Möglichkeit, die auf biologische Unterschiede zurückzuführende Ungleichheit von Männern und Frauen bei der Karriereplanung zu reduzieren (Harwood 2015). Sie argumentieren, dass der Zeitpunkt der Familiengründung die Ausbildung und die Karriere von Frauen beeinflusst und ihre Wahlmöglichkeiten einschränkt. Social Freezing erweitert diese Wahlmöglichkeiten (Goold und Savulescu 2009) und erlaubt es Frauen, "alles zu haben» (Harwood 2009, Richards 2013, Urist 2013, zitiert in Cattapan et al. 2014). Social Freezing wird als Beitrag an die Emanzipation der Frauen gesehen, weil diese ihre Karriere vorantreiben und gleichzeitig genetisch eigene Kinder haben können (Roth 2016).

Kritische Stimmen berufen sich auf ein relationales Autonomieverständnis und befürchten, dass Social Freezing die bestehenden Geschlechterverhältnisse auf dem Arbeitsmarkt zementiert. Sie werfen Befürworterninnen und -befürworter des Social Freezing vor, mit der gleichen Logik wie die feministische Bewegung in den 1960er-Jahren zu argumentieren, welche unter Gleichberechtigung dieselben Chancen für Männer und Frauen auf dem Arbeitsmarkt verstand. Zuerst durch die Pille und nun durch Social Freezing sollen Frauen die Familienplanung zugunsten ihrer Arbeitstätigkeit zurückstellen (Kovács 2013). Das Ideal des männlichen Arbeitnehmers ohne familiäre Verpflichtungen wird durch So- 
cial Freezing nicht infrage gestellt (McGinley 2016, De Graaf 2016). Dieses Ideal wird weder der Realität der heutigen Frauen gerecht, die sich stärker im Beruf engagieren wollen, noch der Männer, die eine wichtigere Rolle in ihrer Familie spielen möchten (De Graaf 2016). Die Lösung gesellschaftlicher Zwänge wird jeder einzelnen Frau überlassen. Statt gesellschaftliche Strukturen zur Förderung der Vereinbarkeit von Beruf und Familie auszubauen, würde ein sozialer Druck auf Frauen ausgeübt, ihren Kinderwunsch in die zweite Lebenshälfte zu verschieben (De Graaf 2016, Linkeviciute et al. 2015, Cattapan et al. 2014, Tobias Eichinger und Uta Bittner 2010, zitiert in Roth 2016). Diese Verschiebung der Familienplanung führt jedoch nicht zu einer Vereinbarkeit von Beruf und Familie, sondern lediglich zu einem zeitlichen Auseinanderdriften dieser beiden Lebenssphären (Roth 2016). Die Unterordnung unter die Erfordernisse des Arbeitsmarktes ist für die Frauen, die ihre Eizellen einfrieren lassen, nicht risikolos. Aufgrund der schwer vorhersehbaren Erfolgsraten des Social Freezing ist es möglich, dass sie gegen ihren Wunsch kinderlos bleiben (Shkedi-Rafid und Hashiloni-Dolev 2012).

Befürworterinnen und Befürworter des Social Freezing bezweifeln aus drei Gründen, dass Social Freezing an die Stelle von Massnahmen zur Förderung von Beruf und Familie treten wird. Sie argumentieren erstens, dass beruflich aktive Mütter auch in fortgeschrittenem Alter noch auf solche Massnahmen angewiesen sein werden (Wunder 2014, Bernstein und Wiesemann 2014, Roth 2016). Zweitens wird von den Kritikerinnen und Kritikern des Social Freezing suggeriert, dass alle Frauen in jungen Jahren Kinder gebären möchten, sobald die gesellschaftlichen Voraussetzungen gegeben sind. Es wird jedoch weiterhin Frauen und Männer geben, die sich erst später bereit für eine Familie fühlen (Bernstein und Wiesemann 2014, Mertes 2013). Drittens wird infrage gestellt, ob Massnahmen zur Vereinbarung von Beruf und Familie den Zeitpunkt der Familiengründung beeinflussen (Dondorp und De Wert 2009). So zeigen Studien, dass solche Massnahmen zwar von Eltern begrüsst werden, sie aber nicht zu mehr Kindern oder jüngeren Eltern führen würden. Sie beeinflussen am ehesten Personen, für welche die Familie sowieso zentral ist und die ihre Karriere zugunsten ihrer Kinder zurückstellen würden (Hakim 2013, zitiert in Mertes 2013). Aus diesen Gründen werden Social Freezing sowie Massnahmen zur Förderung der Vereinbarkeit von Beruf und Familie nicht als antagonistisch, sondern als komplementär erachtet (Mertes 2013, ESHR 2012, Goold und Savulescu 2009). Diese Einstellung vertritt auch die NEK in ihrer Stellungnahme 
zum Social Freezing. Sie spricht sich zwar für Social Freezing aus, fordert aber gleichzeitig Massnahmen zur Vereinbarkeit von Beruf und Familie.

Mit der ausführlichen Diskussion des Zusammenspiels von Social Freezing und der Vereinbarkeit von Beruf und Familie wird dem Thema jedoch eine Bedeutung beigemessen, welche durch die empirischen Befunde nicht gestützt wird. Zwei Gründe können eine Erklärung für die Dominanz dieses Themas in der Diskussion des Social Freezing sein. Einerseits werden die Frauen mit kryokonservierten Eizellen in den Medien primär als Frauen präsentiert, welche die Technologie nutzen, um ihre Karriere voranzutreiben und ihren Kinderwunsch zurückzustellen (Baldwin 2016). Andererseits wurde die öffentliche Diskussion zu diesem Thema wesentlich durch den Entscheid von Facebook und Apple geprägt, ihren Mitarbeiterinnen beziehungsweise den Partnerinnen ihrer Mitarbeiter einen finanziellen Beitrag an das Social Freezing zu leisten (vgl. Kapitel 5.3). Der Einfluss des Social Freezing auf die Vereinbarkeit von Beruf und Familie könnte zunehmen, wenn künftig immer mehr jüngere Frauen zur Karriereplanung auf die Technologie zurückgreifen. Gegenwärtig ist jedoch für die Inanspruchnahme des Social Freezing primär der fehlende Partner ausschlaggebend. Dieser Sachverhalt wird allerdings in den wissenschaftlichen Beiträgen nur anekdotisch diskutiert. Es fehlt sowohl bei den Fürsprechern als auch bei den Kritikern des Social Freezing eine umfassende und systematische Reflexion über das Zusammenspiel von Social Freezing und den Familiengründungsabsichten von Männern und Frauen. Die Hauptursache für dieses Versäumnis dürfte sein, dass Social Freezing und auch die Familiengründung generell in erster Linie als Angelegenheit der Frauen wahrgenommen und diskutiert werden. So gibt es kaum Studien, welche die Familienplanung bei Männern (Perrier 2013) oder die Verhandlung zwischen Paaren vor dem Entscheid, eine Familie zu gründen, untersuchen (Baldwin 2016).

Ein positiver Zusammenhang zwischen Social Freezing und Familiengründung wird mit dem Argument hergestellt, dass Frauen dank dieser Technologie genauso viel Zeit für die Partnersuche und Familiengründung haben wie Männer (Harwood 2015). Weiter wird angeführt, dass Social Freezing eine Antwort auf eine soziale Entwicklung sei, welche beide Geschlechter betreffe. Nicht nur Frauen, sondern auch Männer bekunden Mühe, das kurze Gelegenheitsfenster zwischen der Erreichung von Ausbildungs- und Karrierezielen sowie der Familiengründung zu nutzen (Lookwood 2011). Die Kritikerinnen und Kritiker des Social Freezing werfen Frauen vor, dass sie unter der Illusion leiden würden, für 
immer nach dem Traumprinzen suchen zu können (Shkedi-Rafid und HashiloniDolev 2012). Frauen werden davor gewarnt, die Suche nach einem passenden Partner mittels Social Freezing in unbestimmte Zukunft zu vertagen, weil das Angebot auf dem Heiratsmarkt mit zunehmendem Alter sinkt (Kovács 2013). Es wird gar befürchtet, dass Social Freezing die Verhandlungsmacht der Frauen in einer Partnerschaft senkt. Frauen können ihre Partner mit Verweis auf die biologische Uhr nicht mehr für die Familiengründung gewinnen, sondern ihre Partner können dank Social Freezing die Familiengründung weiter hinauszögern (Roth 2016).

Die vielversprechendste Erläuterung zum Zusammenspiel von Social Freezing und Familiengründungsabsichten beider Geschlechter liefert Baldwin (2016). Sie argumentiert, dass Social Freezing zwar hauptsächlich in Anspruch genommen wird, weil sich Frauen und Männer in der Familienplanung nicht finden. Die Technologie trägt jedoch nicht zu einer Lösung dieser Problemkonstellation bei, sondern belässt die Hauptverantwortung für die Familienplanung bei den Frauen. Frauen, die ihre Eizellen einfrieren liessen, suchen offenbar einen Partner, der sich stark in der Kindererziehung engagiert und die Kinderbetreuung gemeinsam mit der Mutter wahrnimmt. Daten aus dem Familienbericht des Bundesamtes für Statistik deuten allerdings darauf hin, dass dieses Anliegen nicht in gleichem Ausmass von den Männern geteilt wird. In der für Social Freezing relevanten Gruppe der Frauen mit Tertiärbildung erachten 46,4 Prozent die Arbeitsteilung mit ihrem Partner als eine wichtige Voraussetzung für die Familiengründung. Bei Männern mit Tertiärbildung sind nur 26,7 Prozent dieser Ansicht (BFS 2017). Dieser Anteil liegt aber weit über dem Anteil der Männer, die ihr Arbeitspensum effektiv reduzieren. In der Schweiz arbeitet nur jeder zehnte Vater Teilzeit. Lediglich bei 5,9 Prozent der Paare mit Kindern unter 25 Jahren arbeiten beide Elternteile Teilzeit, und bei 11,1 Prozent sind beide voll erwerbstätig. Bei 2,4 Prozent der Eltern ist die Frau Vollzeit und der Mann Teilzeit beschäftigt. In der überwiegenden Mehrheit der Familien arbeitet der Mann Vollzeit (BFS 2017). Gefragt sind entsprechend nicht nur Massnahmen für Frauen, welche die Arbeitstätigkeit von Müttern begünstigen, sondern auch Massnahmen für Väter, die innen ein stärkeres Engagement in der Familie erlauben. 


\subsubsection{Medikalisierung sozialer Probleme}

Social Freezing trägt zu einer Medikalisierung der oben beschriebenen sozialen Probleme bei. Unter Medikalisierung wird die Ausweitung des medizinischen Zuständigkeitsbereichs auf die Lösung gesellschaftlicher Probleme verstanden (Roth 2016, Baldwin 2016, Shkedi-Rafid und Hashiloni-Dolev 2012, Romain 2011). Die Medikalisierung in Zusammenhang mit unerwünschter Kinderlosigkeit wird in der Dissertation von Baldwin (2016) ausführlich beschrieben. Gemäss dieser Autorin wurde die ungewollte Kinderlosigkeit bis in die 1970erJahre hinein als soziales Problem und als ein Schicksal beschrieben, das die Betroffenen ertragen müssen. Mit der Erfindung der Befruchtung von Eizellen in vitro wurde dieses soziale Problem neu beurteilt. Statt von ungewollter Kinderlosigkeit war nun vom medizinischen Problem der Unfruchtbarkeit die Rede. In Zusammenhang mit Social Freezing kann jedoch nicht mehr von einer Medikalisierung, das heisst der Bekämpfung von Krankheiten und der Wiederherstellung der normalen Körperfunktion, gesprochen werden. Stattdessen wird eine Verbesserung beziehungsweise ein «Enhancement» des eigenen Körpers angestrebt und von Biomedikalisierung gesprochen. Social Freezing limitiert sich nicht mehr auf die Bekämpfung der Infertilität, sondern schliesst die «antizipierte Infertilität» mit ein. Diese Kategorie umfasst nicht nur weit mehr Frauen, sondern führt auch zu einer Neubeurteilung der reproduktiven Alterung. Diese wird nicht mehr als natürlicher Prozess, sondern als medizinisches Problem interpretiert, welches dank Social Freezing angegangen werden kann.

Während die (Bio-)Medikalisierung gesellschaftlicher Probleme eine Ausweitung reproduktionsmedizinischer Verfahren begünstigt, werden medizinische Argumente herangezogen, um die Nutzung reproduktionsmedizinischer Verfahren einzuschränken. So hob die amerikanische Gesellschaft für Reproduktionsmedizin (ASRM) im Jahr 2012 zwar den experimentellen Status für die Eizellkonservierung auf, empfahl jedoch, diese Technologie nur bei Krebspatientinnen anzuwenden. Da die Datenlage zur Sicherheit, Wirksamkeit und Effizienz der Technologie als ungenügend eingestuft wurde, lehnte die ASRM die Eizellkonservierung aus sozialen Gründen ab. Die Unterscheidung zwischen medizinischen und sozialen Gründen beim Zulassungsentscheid sorgte für viel Diskussionsstoff. Gemäss Pennings (2013) werden medizinische Gründe herangezogen, um gerechtfertigte von nicht gerechtfertigten Interventionen zu unterscheiden. Social Freezing wird entsprechend mit anderen Wahleingriffen wie Schönheitsoperationen verglichen, die nicht auf einer therapeutischen Notwen- 
digkeit, sondern lediglich auf dem Wunsch der Betroffenen beruhen. Allerdings erweist sich die Unterscheidung zwischen sozialen und medizinischen Gründen im Zugang zur Eizellkonservierung aus verschiedenen Gründen als schwierig. Erstens ist nicht nur beim Social Freezing, sondern auch bei der In-vitroBefruchtung generell das Alter der Frau eine der häufigsten Ursachen für die Unfruchtbarkeit. Trotzdem steht einer Behandlung dieser Frauen nichts entgegen (EHSR 2012, Académie nationale de médecine 2017, Pennings 2013, Dondorp und De Wert 2009, Mertes 2013). Zweitens werden gerade im Bereich der Fortpflanzung mit Verhütung, Sterilisation und Abtreibung verschiedene Eingriffe vorgenommen, die nicht aus medizinischen, sondern aus sozialen Gründen erfolgen (ESHR 2012, Académie nationale de médecine 2017, Mertes und Pennings 2012). Drittens zeigen Studien, dass eine Minderheit der Frauen zwar aufgrund eines fehlenden Partners ihre Eizellen einfrieren lässt, gleichzeitig aber unter einer eingeschränkten Fruchtbarkeit beispielsweise infolge einer Endiometriose leidet (Baldwin 2016). Die Inanspruchnahme der Technologie kann somit weder auf rein soziale noch primär medizinische Gründe zurückgeführt werden (Belaisch-Allart et al. 2013). Viertens wird bestritten, dass Social Freezing keinen therapeutischen Nutzen hat. Gute Medizin umfasst nicht nur die Behandlung, sondern auch die Prävention von Krankheiten. Die Antizipation einer ungewollten Infertilität wird in diesem Sinne als präventive Medizin gesehen (ESHR 2012, Mertes und Pennings 2012, Harwood 2015). Eine solche Definition begünstigt allerdings wiederum eine Ausweitung reproduktionsmedizinischer Verfahren und die Medikalisierung sozialer Probleme.

\subsubsection{Familienbilder}

Der Einfluss des Social Freezing auf die Familienbilder ist in der wissenschaftlichen Literatur kein dominantes Thema. Einerseits wird diskutiert, wie sich Social Freezing auf das dominante, auf naturalistische Vorstellungen und heterosexuelle Eltern gestützte Familienbild auswirkt. Andererseits steht der Einfluss des Social Freezing auf die Generationenbeziehungen zur Diskussion. 
Die Erzählungen der Frauen mit kryokonservierten Eizellen unterstreichen die Bedeutung, die diese Frauen einer genetischen Verbindung zwischen beiden Elternteilen und dem Kind beimessen (vgl. Kapitel 3.1.3). Die Nutzung dieser Technologie spiegelt entsprechend die Orientierung an einem dominanten naturalistischen Familienbild (Harwood 2015, Cutas und Smajdor 2015). Es wird aus diesem Grund als verständlich erachtet, dass sich diese Frauen alternative Familienformen wie die Adoption eines Kindes oder die Zeugung eines Kindes mittels Spermienspende nicht vorstellen können (Dondorp und De Wert 2009, De Graaf 2016, Cutas und Smajdor 2015). Gleichzeitig besteht die Befürchtung, dass Social Freezing dazu führt, dass der genetischen Elternschaft noch mehr Bedeutung zugemessen wird (Cutas und Smajdor 2015) und alternative Familienformen weniger akzeptiert oder gar stigmatisiert werden (Linkeviciute et al. 2015). Weiter wird kritisiert, dass eine genetische Elternschaft nicht hinterfragt und als gut erachtet wird (Harwood 2015). Eine zunehmende Anzahl Forschungsarbeiten zeigt, dass eine genetische Verbindung zwischen Eltern und Kindern weder eine Garantie für eine gelungene Eltern-Kind-Beziehung darstellt noch eine notwendige Voraussetzung dazu ist. Einzelne Forschende gelangen gar zum Schluss, dass die Kindererziehung von Eltern ohne genetische Verbindung zu ihren Kindern besser ist (Cutas und Smajdor 2015).

Mit dem Wunsch nach einer genetischen Elternschaft entsprechen die Frauen, die ihre Eizellen einfrieren lassen, zwar einer gesellschaftichen Norm - nicht aber mit der Familiengründung in fortgeschrittenem Alter (Harwood 2015). Mütter über 50 Jahren stossen in der Gesellschaft auf Widerstand, weil sie im als unnatürlich erachteten hohen Alter eine Familie gründen (Kress 2013) und somit implizite Natürlichkeitsvorstellungen verletzen (Roth 2016). Die gesellschaftliche Reaktion auf diese Verletzung gesellschaftlicher Normen manifestiert sich in den Erzählungen von älteren Eltern. Diese sind mit negativen Reaktionen ihres sozialen Netzwerkes konfrontiert, die von einer reduzierten Unterstützung durch Freunde bis hin zu gesellschaftlichen Sanktionen reichen können und mit einer sozialen Isolierung der Eltern verbunden sind. Auch auf dem Spielplatz und in den Schulen sind die Eltern mit einer Stigmatisierung konfrontiert und befürchten eine solche für ihren Nachwuchs (Woodward und MacDuffie 2016). Gleichzeitig sind auch andere neuartige Familienkonstellationen wie die Elternschaft lesbischer Paare mittels Spermienspende in der Natur nicht vorgesehen. Social Freezing ist entsprechend nur ein Element eines umfassenderen Wan- 
dels von Familienbildern (Kress 2013) und stellt in diesem Kontext möglicherweise eine gewinnbringende Veränderung dar (Roth 2016).

\section{Einfluss des Social Freezing auf die Generationenbeziehungen}

Die Autorinnen und Autoren weisen darauf hin, dass Social Freezing ebenfalls die Generationenbeziehungen beeinflusst. Wenn Frauen dank der kryokonservierten Eizellen sehr spät Mütter werden, sind die Grosseltern ihrer Kinder häufig bereits pflegebedürftig oder gar verstorben. Die Kinder können nicht von einer wertvollen Beziehung zu ihren Grosseltern profitieren (Linkeviciute et al. 2015). Statt in einen Familienverbund mit drei Generationen eingebunden zu sein, wachsen die Kinder in der Anwesenheit von lediglich zwei Generationen auf (Roth 2016). Die Kinder von älteren Müttern müssen möglicherweise nicht nur auf Grosseltern, sondern auch auf Geschwister verzichten (Woodward und MacDuffie 2016). Wenn, wie empfohlen, 15 bis 20 Eizellen eingefroren werden, kann damit in der Regel eine Lebendgeburt erzielt werden (vgl. Kapitel 2.3.2). Die Schwangerschaftsrisiken steigen zudem mit zunehmendem mütterlichen Alter. Dies kann zu einer reduzierten Anzahl Geschwister führen, weil das Zeitfenster für eine komplikationslose Schwangerschaft und Geburt abnimmt.

Die auseinandergezogene Generationenfolge hat auch Konsequenzen für die Pflegearbeit zwischen den Generationen. Die Grosseltern können die Mütter nicht bei der Kindererziehung entlasten (Wunder 2013), sondern sorgen im Gegenteil durch ihre eigene Pflegebedürftigkeit für eine Doppelbelastung der Mütter, die sich gleichzeitig um ihre kleinen Kinder und ihre betagten Eltern kümmern müssen (Roth 2016). Dieses Schicksal dürften auch die mittels kryokonservierter Eizellen gezeugten Kinder teilen. Sie müssen möglicherweise bereits in jungen Jahren die Pflege ihrer Eltern übernehmen und können dafür weniger in ihre Ausbildung, Karriere und Liebesbeziehungen investieren (Woodward und MacDuffie 2016, Goold und Savulescu 2009). Wenn die Kinder als Einzelkinder aufwachsen, können sie sich die Pflegearbeit nicht mit ihren Geschwistern teilen. Auf der anderen Seite dürften Frauen, die spät Mutter wurden, über mehr finanzielle Ressourcen verfügen und für ihre Pflege aufkommen können. Die wesentlich jüngeren Kinder dürften zudem noch keine eigenen Gesundheitsprobleme haben und daher besser für ihre betagten Eltern sorgen können als Kinder, die selber bereits älter sind (Goold und Savulescu 2009). 


\subsection{Diskussion der finanziellen Dimension des Social Freezing}

Die Kosten des Social Freezing werden zurzeit hauptsächlich von den Frauen getragen. In diesem Zusammenhang wird diskutiert, ob die Inanspruchnahme dieser Technologie wirklich im Interesse der Frauen oder nicht eher der Anbieter ist. Für reichlich Diskussionsstoff haben zudem grosse amerikanische Technologiefirmen wie Apple und Facebook gesorgt, die ihren Mitarbeiterinnen anbieten, die Kosten für Social Freezing zu übernehmen. Demgegenüber wird der Übernahme der Kosten für Social Freezing durch das öffentliche Gesundheitssystem in den Diskussionen weniger Platz eingeräumt.

\subsubsection{Private Finanzierung}

Social Freezing ist relativ kostspielig. In der Schweiz belaufen sich die Kosten für die Hormonstimulation, Eizellentnahme und Kryokonservierung auf zwischen 3000 und 6000 Franken pro Stimulationszyklus. Für die Lagerung muss mit 200 bis 300 Franken pro Jahr gerechnet werden. Für die Eizellbefruchtung und den Embryotransfer müssen zwischen 2500 und 3500 Franken veranschlagt werden (Wunder 2013). In Deutschland fallen Kosten von rund 3000 Euro, in Grossbritannien von 4000 bis 5000 Pfund und in den USA von 6000 bis 18'000 Dollar an (Linkeviciute et al. 2015).

Die Frauen investieren relativ viel Geld in ein Verfahren, dessen Erfolgschancen zum jetzigen Zeitpunkt nicht klar bestimmt werden können (vgl. Kapitel 2.3.2). Mehrere Autorinnen und Autoren stellen infrage, ob Social Freezing wirklich einem Bedürfnis der Frauen entspricht und die Nachfrage nicht eher durch die Anbieter dieser Technologie erzeugt wurde. Diesen wird vorgeworfen, aus finanziellen Interessen ein soziales Problem zu medikalisieren und zu bewirtschaften (Kress 2013, Roth 2016, Shkedi-Rafid und Hashiloni-Dolev 2012). Als Illustration für die Bedeutung kommerzieller Interessen werden die in amerikanischen und englischen Grossstädten populären Egg-Freezing-Partys herangezogen. Bei Champagner sollen die Frauen an diesen Werbeanlässen von den Vorteilen dieser Technologie überzeugt werden (Mertes 2017, McGinley 2016). Um kommerzielle Interessen zu unterbinden, schlägt die Académie national de 
médecine (2017) in Frankreich vor, dass Social Freezing nur in öffentlichen, gemeinnützigen Kliniken angeboten werden soll, welche über die notwendige Erfahrung für eine erfolgreiche Anwendung der Technologie verfügen.

Es gibt jedoch auch einzelne Stimmen, die diese Einwände als bevormundend und sexistisch empfinden. Die Frauen können die Risiken des Verfahrens durchaus verstehen (McGinley 2016) und sollen wie in allen Lebensbereichen auch bezüglich des Social Freezing allein finanzielle Entscheidungen treffen können (Goold und Savulescu 2009). Nur selten bedauern die Frauen die Nutzung des Social Freezing (vgl. Kapitel 3.1.4) oder eines anderen reproduktionsmedizinischen Verfahrens, selbst wenn die Behandlung nicht erfolgreich ist. Von grösserer Bedeutung ist für die Frauen, alle Möglichkeiten zur Realisierung ihres Kinderwunsches ausgeschöpft zu haben (Mertes 2017).

Um den Einfluss der Anbieter auf die Nachfrage nach Social Freezing zu beurteilen, bieten sich zwei Konzepte aus der Gesundheitsökonomie an. So könnte beim Social Freezing einerseits eine angebotsinduzierte Nachfrage spielen. Eine solche angebotsinduzierte Nachfrage entsteht durch ein Informationsgefälle zwischen Arzt und Patient, das dem Arzt erlaubt, die Nachfrage nach seinen Leistungen aus kommerziellen Interessen zu beeinflussen. Je mehr Ärztinnen und Ärzte medizinische Leistungen erbringen, desto häufiger werden sie auch bei fraglicher oder fehlender Indikation angewendet. Beim Social Freezing fehlt grundsätzlich eine medizinische Indikation, die das kommerzielle Interesse der Reproduktionsmediziner einschränken könnte. Zudem findet die Beratung zum Social Freezing im Rahmen einer Arzt-Patientin-Beziehung statt, in der kommerzielle Aspekte von beiden Seiten leicht ausgeblendet werden. Bisher liegen keine Studien vor, welche eine angebotsinduzierte Nachfrage beim Social Freezing untersuchen. Die empirischen Untersuchungen im Rahmen dieser Studie deuten allerdings darauf hin, dass in den Fertilitätskliniken durchaus Kapazitäten für eine Leistungsausweitung mittels Social Freezing vorhanden sind. Andererseits spielen beim Social Freezing keine Fehlanreize, welche durch die Übernahme der Kosten von medizinischen Leistungen durch die Krankenkassen entstehen (moral hazard). Die Frauen kommen für die effektiven Kosten für Social Freezing auf und müssen nicht, wie bei kassenpflichtigen Leistungen, lediglich die Versicherungsprämien begleichen. Sie haben entsprechend wenig Anreiz, mehr Leistungen nachzufragen als notwendig. Die Kosten sind auch der wichtigste Faktor, welche im Rahmen dieser Studie befragte potenziell interes- 
sierte Frauen die Inanspruchnahme des Social Freezing erschweren würde (vgl. Kapitel 4.2.2).

\subsubsection{Finanzierung durch die Arbeitgeber}

Die Ankündigung von Apple und Facebook, ein allfälliges Social Freezing von Mitarbeiterinnen mit 20'000 Dollar zu entgelten, hat ein weltweites Medienecho ausgelöst (Harwood 2015, Roth 2016). Andere Unternehmen wie Intel oder auch das amerikanische Verteidigungsdepartement folgten dem Beispiel der beiden Tech-Giganten. Die Unternehmen wollten damit unter anderem ihre Attraktivität als Arbeitgeber bei bereits angestellten und künftigen weiblichen Arbeitskräften stärken. Social Freezing ist in der Tech-Industrie Bestandteil eines für amerikanische Verhältnisse grosszügigen Katalogs an Unterstützungsleistungen, welche ein Kindergeld bei der Geburt von 4000 Dollar, einen 14-wöchigen Mutter- beziehungsweise Vaterschaftsurlaub sowie die Übernahme der Kosten für eine In-vitro-Befruchtung, Adoption und Leihmutterschaft umfassen. Die Tech-Unternehmen zeigen sich an technologischen Lösungen interessiert, die zu positiven sozialen und wirtschaftlichen Veränderungen für Individuen führen (Grant 2016).

Solange Social Freezing Bestandteil eines solchen Katalogs familienfreundlicher Leistungen ist und kein Druck zur Nutzung dieser Technologie besteht, wird das Angebot dieser Unternehmen durchaus als Möglichkeit gesehen, die reproduktive Autonomie der Frauen zu stärken. Durch den Arbeitgeberbeitrag wird ein Anreiz geschaffen, möglichst früh Eizellen einfrieren zu lassen. Die Frauen warten dadurch nicht mit der Kryokonservierung ihrer Eizellen zu, bis sich Probleme bei der Familiengründung abzeichnen und ein Kinderwunsch nur noch mit kryokonservierten Eizellen aufrechterhalten werden kann. Dadurch dürften die Eizellqualität und in der Folge die Erfolgsaussichten des Verfahrens besser sein. Schliesslich profitieren die Unternehmen auch davon, dass die Frauen die Familiengründung verschieben (Mertes 2017).

Die meisten Autorinnen und Autoren äussern sich jedoch deutlich kritischer. Sie befürchten, dass die reproduktive Autonomie der Frauen durch die Arbeitgeberbeiträge am Social Freezing eingeschränkt wird. Unterschwellig wird innen vermittelt, dass sie die Familiengründung nach hinten verschieben sollen, wenn 
innen die berufliche Karriere wichtig ist (Harwood 2015). Der Beruf wird entsprechend höher gewertet als das Familienleben. Statt Social Freezing anzubieten, fordern die Autorinnen die Unternehmen auf, Massnahmen zur Vereinbarkeit von Beruf und Familie zu ergreifen (McGinley 2016, Bricknell 2016, Datta 2017, De Graaf 2016).

Obwohl zu diesem Thema viele Artikel publiziert wurden, sind die Diskussionen für die Schweiz aus drei Gründen nicht besonders relevant. Erstens dürfte die Beteiligung von Facebook und Apple an den Kosten ihrer Mitarbeiterinnen für Social Freezing eine Randerscheinung bleiben und keine breite Anwendung bei anderen Unternehmen ausserhalb dieses sehr technologieaffinen Umfelds finden (Harwood 2015). Zweitens nehmen Frauen Social Freezing nicht primär zur Vereinbarkeit von Beruf und Familie in Anspruch, sondern um mehr Zeit für die Partnersuche zu gewinnen. Drittens ist die Übernahme der Kosten für Social Freezing in den USA vor dem Hintergrund des dortigen Gesundheitswesens zu verstehen. Amerikanische Unternehmen schliessen für ihre Angestellten eine Krankenversicherung ab und definieren den Leistungskatalog (Roth 2016). In Europa haben die Arbeitgeber keine solche Rolle und die Kostenübernahme würde eher über das öffentliche Gesundheitswesen beziehungsweise in der Schweiz im Rahmen des Krankenversicherungsgesetzes erfolgen. Allerdings sind die amerikanischen Unternehmen auch in der Schweiz tätig, und es ist unklar, ob auch die Schweizer Arbeitnehmerinnen von der Kostenbeteiligung dieser Firmen profitieren.

\subsubsection{Finanzierung durch die öffentliche Hand}

Bisher ist Israel das einzige Land, in dem die Kosten für Social Freezing durch die Krankenkassen gedeckt sind (Linkeviciute et al. 2015). In der Schweiz stellt sich die Frage der Kostenübernahme im Rahmen des Krankenversicherungsgesetzes erst dann, wenn die Befruchtung in vitro krankenkassenpflichtig wird. Relativ unbestritten scheint, dass die Kosten für die Eizellentnahme und -konservierung auch künftig von den Frauen beglichen werden sollten, weil die künftige Nutzung der eingefrorenen Eizellen ungewiss ist. Falls die Frauen die Eizellen aufgrund einer altersbedingten Unfruchtbarkeit im Rahmen einer IVFBehandlung befruchten lassen, sollen die bereits geleisteten Beiträge an Eizellentnahme und -konservierung aus zwei Gründen angerechnet werden (ESHR 
2012, Mertes und Pennings 2012, Belaisch-Allart et al. 2013). Einerseits ist es eine Frage der Gerechtigkeit, dass für Frauen, die in jungen Jahren ihre Eizellen einfrieren liessen und nun altersbedingt unfruchtbar sind, die gleichen finanziellen Bedingungen gelten wie für andere unfruchtbare Patientinnen. Die Behandlungen sollten im öffentlichen Gesundheitswesen zumindest bis Anfang 40 abgegolten werden (ESHR 2012). Andererseits ist Social Freezing im Vergleich zu anderen Verfahren kosteneffizienter. Wenn Eizellen verwendet werden, die in jungen Jahren entnommen wurden, ist die Geburtenrate höher, die Fehlgeburtenrate tiefer und die Kinder leiden unter weniger Chromosomenanomalien. Im Vergleich zu einer Fruchtbarkeitsbehandlung in fortgeschrittenem Alter sind weniger Stimulationszyklen notwendig (Mertes und Pennings 2012). 


\section{Diskussion des Social Freezing in den Schweizer Medien}

In diesem Kapitel wird die Diskussion des Social Freezing in den Medien beschrieben. Insgesamt wurden 44 Artikel analysiert, welche zwischen 2011 und 2017 in Schweizer Medien und Fachzeitschriften publiziert wurden. Der Grossteil der Artikel wurde im Jahr 2014 veröffentlicht. Dies lässt sich damit erklären, dass damals bekannt wurde, dass sich US-amerikanische Firmen wie Facebook und Apple an den Kosten für das Einfrieren von Eizellen ihrer Mitarbeiterinnen beteiligen. Entsprechend wurde diese Thematik auch in den Schweizer Medien aufgearbeitet. Andere Anlässe, auf die sich das Erscheinen von Artikeln zur Thematik Social Freezing in Schweizer Medien zurückführen lässt, sind politische Diskussionen zum Schweizer Fortpflanzungsmedizingesetz (z.B. Umsetzung der parlamentarischen Initiative zur Zulassung der Eizellspende, Diskussion zur Aufbewahrungsfrist von Eizellen), Studienergebnisse zu medizinischen Risiken von Reproduktionstechniken sowie Einstellungen der Schweizer Bevölkerung zur Fortpflanzungsmedizin, ein Anstieg der Anzahl Schweizer Patientinnen in Reproduktionskliniken in Spanien sowie juristische Interventionen gegen die Werbung von Privatkliniken zur Eizellenvorsorge.

Die Diskussionen in den Medien werden häufig durch Expertinnen und Experten betrieben, die in den Medienartikeln zitiert werden. Die am häufigsten zitierten Expertenmeinungen stammen von Reproduktionsmedizinerinnen und -medizinern grosser Schweizer Spitäler (wie CHUV, Inselspital Bern) oder spezialisierten Reproduktionskliniken (z.B. Ovita). Für die Diskussion der gesellschaftlichen Dimension des Social Freezing werden in den Artikeln zudem Meinungen von geistes- und sozialwissenschaftlichen Expertinnen und Experten, beispielsweise aus den Bereichen der Philosophie, Ethik, Kultur- oder Politikwissenschaften, hinzugezogen. Vereinzelt kommen Politikerinnen und Politiker zu Wort, die zu aktuellen politischen Fragen Stellung nehmen. Kaum zitiert werden jedoch Frauen, die diese Technik bereits angewendet haben oder sich dafür interessieren.

Bei der Mehrheit der untersuchten Medienartikel lässt sich keine klar positive oder negative Haltung zum Social Freezing erkennen. Die meisten der in den 
Artikeln zitierten Meinungen befürworten weder ein vollständiges Verbot des Social Freezing noch eine völlig liberale Handhabung ohne jegliche staatliche Regulierung. Meist werden in den Artikeln sowohl Vor- wie auch Nachteile der Technik erläutert. Nachfolgend werden die wichtigsten Aussagen zur Nachfrage nach Social Freezing sowie zur ethischen, gesellschaftlichen, finanziellen und rechtlichen Dimension dieser Technologie dargelegt.

\subsection{Diskussion der ethischen Dimension in den Schweizer Medien}

Die ethische Diskussion fokussiert in den untersuchten Medienartikeln auf die Abwägung zwischen Kindeswohl und der Fortpflanzungsautonomie der Frau. In elf Artikeln finden sich entsprechende Aussagen. In den Artikeln wird mehrmals betont, dass die Autonomie der Frau als ein wesentliches Gut anzusehen ist und Social Freezing - ähnlich wie die Pille oder IVF - als eine Möglichkeit zur Steigerung der Autonomie der Frau gesehen werden kann.

"Ethiker sehen die Autonomie der Frau als ein wesentliches Gut an, aufgrund derer sie selber entscheiden kann, ob sie Oozyten konservieren möchte oder nicht. [...] Da die Pille und die IVF inzwischen aus ethischer Sicht weitgehend akzeptiert sind, wäre es ein Widerspruch, das «Social freezing pauschal als ethisch nicht zulässig zu verurteilenı.» (Schweizerische Ärztezeitung, 2013)

"Es gibt keine guten Gründe, einer gut informierten Frau diese Möglichkeit zu verwehren, nur weil sie nicht an Krebs erkrankt ist. [...] Die Möglichkeit der Vitrifikation ist ein Schritt zur Emanzipation, der - nur weil er durch den technischen Fortschritt bedingt ist - nicht weniger nachhaltig und segensreich sein muss als andere Fortschritte.» (Tages-Anzeiger, 2011)

Grenzen der Reproduktionsmedizin werden da gezogen, wo das Kindeswohl bedroht ist, denn das Kindeswohl sei das oberste Gut und der wichtigste Grundsatz bei der Anwendung von fortpflanzungsmedizinischen Verfahren. Die potenziellen medizinischen Risiken für das Kind werden in in den Medien kontrovers diskutiert. In vielen Artikeln werden mögliche Risiken für das Kindeswohl aufgezählt - sowohl physischer Art (wie Fehlbildungen, Veranlagung für Diabe- 
tes und Herz-Kreislauf-Probleme) wie auch psychischer Art («Precious Baby Syndrome»). Oftmals wird betont, dass es an Langzeitstudien und damit an den notwendigen Daten fehle.

"Vielleicht nehmen auch Vorstellungen von Planbarkeit und Kontrolle auf eine Art und Weise zu, die das Kindeswohl gegenüber elterlichen Wünschen in den Hintergrund treten lassen. Weil das Kind als Produkt - und nicht mehr als Geschenk - betrachtet wird.» (NZZ am Sonntag, 2015, N. Streeck - Journalistin/Theologin)

In vielen Artikeln werden mögliche negative Auswirkungen eines grossen Altersunterschiedes zwischen Eltern und Kind auf das Kindeswohl diskutiert (z.B. grössere Wahrscheinlichkeit von Halb- und Vollwaisen).

«[...] man sollte eine Alterslimite für künstliche Befruchtung einführen, und zwar ganz allein im Hinblick auf das Kindeswohl (werden früh Halb- oder Vollwaisen).» (Annabelle, 2015, B. Bleisch - Philosophin)

Insgesamt überwiegt in den untersuchten Artikeln die Meinung, dass Kinder auch vom fortgeschrittenen Alter ihrer Eltern profitieren können - sei es dank einer stabileren Beziehung, einer besseren finanziellen Situation oder der grösseren elterlichen Lebenserfahrung. Dennoch kann aus der Mediendiskussion kein Konsens für ein Recht auf ein Kind in jedem Lebensalter abgeleitet werden. Die Mehrheit der Artikel kommt zum Schluss, dass Extremformen im Sinne von Schwangerschaften jenseits der natürlichen Grenze der Fruchtbarkeit problematisch seien. Daher wird oftmals sowohl aus medizinischen wie auch ethischen Überlegungen für eine Alterslimite bei der Frau plädiert. In zwei Artikeln wird auf den interessanten Umstand hingewiesen, dass das Alter der Väter im Gegensatz zu demjenigen der Mütter in der Gesellschaft kaum als Problem wahrgenommen wird.

"Während hierzulande jedes Jahr 15'000 Männer zwischen 40 und 50 frischfröhlich Vater werden, muss sich eine Frau den Vorwurf von Egoismus und Verantwortungslosigkeit gefallen lassen, wenn sie um die 40 ihren Wunsch nach einem Kind noch erfüllen möchte.» (Tages-Anzeiger, 2016, A. Wirthlin - Journalistin)

Allgemein wird Social Freezing als Symptom der heutigen Gesellschaft erkannt, in der Aspekte wie die Kontrolle und Planbarkeit des Lebens einen hohen Stellenwert haben. 
«Beängstigender als dass ich bald nur noch ein paar Eizellen übrig habe, finde ich nämlich die Tatsache, dass sich die Frau mit dem ‘Freezing〉 das Leben quasi zu einem bestimmten Zeitpunkt bestellen kann, ähnlich wie ein Eis-Dessert im Restaurant aus dem Tiefkühler. Das Leben wird berechenbar.» (Basler Zeitung, 2014, R. Koerfgen - Journalistin)

«Mit sexueller Revolution hat social freezing gar nichts mehr zu tun. Sondern mit dem, was der Philosoph Robert Pfaller die ‘Angst vor dem Genuss〉 nennt. Der Idee der kalten Perfektion wird dann alles geopfert. Darin sind die Frauen übrigens die perfekten Mittäter. Eine junge, gesunde Frau, die ihre Eier einfrieren lässt, ist dem islamistischen Selbstmordattentäter strukturell ähnlicher, als sie sich dies je eingestehen würde. Beide dienen einem System, das den eigenen Körper als Mittel für einen übergeordneten Zweck - Privatwirtschaft hier, Allahs Reich dort - vorbehaltslos zur Verfügung stellt.» (Weltwoche, 2014, R. Stämpfli Politologin)

Die ethische Abwägung wird in der Mediendiskussion oft davon abhängig gemacht, wie sehr die Selbstbestimmung der Frau durch externe Faktoren eingeschränkt wird. In vielen Artikeln ist von der Gefahr eines wachsenden sozialen Drucks zur Anwendung des Social Freezing die Rede, der auf die Frau ausgeübt wird, um damit das gesellschaftliche Problem der Vereinbarkeit von Beruf und Familie zu lösen. Als ethisch bedenklich wird zudem die Werbung von Kliniken angesehen, die bei den Frauen falsche Hoffnungen schüren, um ökonomische Interessen zu verfolgen.

"La publicité sur Internet de centres de conservation des ovocytes, comme Ovita pour la Suisse, laisse penser que la femme peut s'affranchir de l'horloge biologique en recourant à ces procédés.» (Le Matin, 2014)

"Studien haben gezeigt, dass Frauen und mit ihnen evtl. auch ihre Partner die voranschreitende Unfruchtbarkeit unterschätzen und gleichzeitig die Möglichkeiten der In-vitro-Fertilisation im Alter überschätzen. Das Schüren von allzu grossen Hoffnungen bei Frauen, die sich erst mit Ende 30 und damit aus medizinischer Sicht zu einem suboptimalen Zeitpunkt mit Social Freezing befassen, ist sicherlich ethisch bedenklich.» (Jusletter, 2015, C. Peter - Jurist) 
"Am Social Freezing frappiert inn [A. Bernard - Kulturwissenschaftler], wie nun auch die Familiengründung in den Bereich der Vorsorge fällt: «Wenn sich das durchsetzt, wird man der 41-Jährigen, die nicht mehr schwanger wird, vorwerfen, sie sei selbst schuld), sagt er. Hätte sie halt lieber Eizellen eingefroren. So denken wir typischerweise heute: Wäre er doch zur Krebsvorsorge gegangen. Hätte sie beim Velofahren einen Helm aufgesetzt. Wir haben Angst vor dem Unvorhergesehenen, Ungeplanten. Social Freezing schenkt das Gefühl von Sicherheit und Kontrolle.» (NZZ am Sonntag, 2015)

"'Was ist sozial an Social Freezing? Bei Facebook und Apple ist schwanger sein nur ein Störfaktor), sagt Wolfgang Bürgstein, Generalsekretär von Justitia \& Pax. ¿Sie setzen die Frauen unter Druck. Damit wird die Reproduktion dem Diktat und den Interessen der Wirtschaft unterstellt.) Dies sei ein ethisches Armutszeugnis.» (Pfarreiblatt der Bistumskantone Schaffhausen und Thurgau, 2014)

In den Artikeln wird nur selten ein liberales Autonomieverständnis vertreten in dem Sinne, dass sich Frauen im heutigen Umfeld selbstbestimmt für Social Freezing entscheiden können.

«Keine Frau trifft eine Entscheidung zum Social Freezing leichtfertig, dafür ist es ein zu unangenehmer Prozess. Er ist wohl überlegt, und eine Garantie auf Erfolg gibt es nicht. Was spricht denn dagegen, zu versuchen, das Beste aus seinem Leben herauszuholen? Schliesslich schadet eine Frau, die sich dazu entschliesst, niemandem damit. Im Gegenteil, sie praktiziert eine verantwortungsbewusste Familienplanung, sie tritt aus der Opfer- und Verzichtrolle heraus und sie nimmt ihr Leben selbst in die Hand. [...] Eine Frau dafür zu verurteilen, weil sie sich den alten Bewertungsschablonen entzieht und ihr Leben selbst in die Hand nimmt, halte ich für zutiefst unseriös und verletzend für die Entscheidungsträgerinnen.» (Basler Zeitung, 2015, F. Lauer - Journalistin)

In den untersuchten Medien findet keine Diskussion statt zur ethischen Vertretbarkeit der vorsorglichen Eizellentnahme mit anschliessender Vernichtung der Eizellen oder zur Möglichkeit, entnommene Eizellen für eine Spende oder die Forschung zur Verfügung stellen zu können. 


\subsection{Diskussion der gesellschaftlichen Dimen- sion des Social Freezing in den Schweizer Medien}

Wie bereits erwähnt, war die Bekanntmachung der Beteiligung an den Kosten des Social Freezing durch Unternehmen einer der Hauptanlässe für die untersuchten Medien, über Social Freezing zu berichten. Dementsprechend nimmt die Diskussion darüber, welchen Beitrag das Social Freezing zur Vereinbarkeit von Beruf und Familie und zu einer verbesserten Chancengleichheit hat, den mit Abstand grössten Raum ein in der Mediendiskussion. In 21 Artikeln wird diese Diskussion aufgegriffen. Der Beitrag des Social Freezing zur Lösung dieser sozialen Probleme wird in den Artikeln mit ganz wenigen Ausnahmen sehr kritisch beurteilt.

«Pour Dorothea Wunder [Reproduktionsmedizinerin], le «social freezing» n'est en tout cas pas la solution aux problèmes de travail. Ce qu'il faudrait avant tout, seIon elle, c'est créer des conditions sociétales permettant à la femme de concilier vie professionnelle et vie de famille.» (Le Temps, 2012)

"Gleichzeitig ist die Beteiligung (von Apple und Facebook) an den Kosten für das ‘Social Freezing) eine Kapitulation, weil sie deutlich macht, dass es opportuner ist, weibliche Eizellen zu konservieren als männliche Karrierestrukturen aufzubrechen.» (NZZ am Sonntag, 2014, N. Althaus - Journalistin)

Im Gegenteil, viele der zitierten Expertinnen und Experten wie auch der Autorinnen und Autoren der Artikel teilen die Überzeugung, dass die Etablierung des Social Freezing in der Gesellschaft es sogar schwieriger machen würde, gesellschaftliche Veränderungen anzustreben. Häufig wird die Befürchtung geäussert, dass Schwangerschaften von Unternehmen zukünftig als "vermeidbar» angesehen und die Anwendung des Social Freezing zu einer sozialen Vorgabe werden könnte: Frauen sollen ihre Schwangerschaft auf später verschieben müssen, wenn sie sich noch beruflich weiterentwickeln möchten. Die Bereitschaft von Unternehmen, Fördermassnahmen wie flexiblere Arbeitszeiten, verlängerten Vaterschaftsurlaub oder subventionierte Kinderbetreuung zu unterstützen, könnte weiter abnehmen. Die Ungleichheit der Geschlechter würde damit noch vergrössert. 
"Vielmehr droht eine gewisse Instrumentalisierung der Frau und ihrer Lebenspläne, die - durch die Möglichkeiten des Social Freezing - nicht wirklich freier geworden ist, denn die Prioritäten werden klar definiert: zuerst Karriere, dann Kinder. Mit dieser Vorstellung, die dem Social Freezing zu Grunde liegt, droht aber letztlich die gesellschaftliche Entwicklung, die Vereinbarkeit von Beruf und Familie durch gleichstellungsrechtliche Ansätze zu erreichen, ernsthaft gehemmt zu werden.» (Jusletter, 2015)

«Es wird Druck auf Frauen ausgeübt, nicht jung schwanger zu werden. Damit wird die Diskriminierung von Frauen verstärkt - was geschehe mit denjenigen, die «normal Kinder haben wollten? Einmal mehr entscheiden vornehmlich (Wirtschafts-)Männer für und über Frauen. Kinder sind nicht einfach das «Restprodukt) einer persönlichen Karriere. Im «Social Freezing) sieht er die Folge des neoliberalen (Denk-)Systems, das den Familien den Support verweigert.» (Pfarreiblatt der Bistumskantone Schaffhausen und Thurgau, 2014, T. Wallimann Theologe)

«Der Theologe und Ethiker Frank Mathwig findet die Vorstellung, dass ein Arbeitgeber Social Freezing finanziert und damit in die Fortpflanzungsfreiheit der Frau eingreift, unerträglich. «Es könnte auf diese Weise ein neues, bisher unbekanntes Diktat der Arbeit eingeführt werden), meint der Professor, der an der Universität Bern lehrt und Mitglied der Nationalen Ethikkommission ist. Zwar bieten Apple und Facebook den Dienst bloss an. Doch was ist, wenn eine Mitarbeiterin dieses Angebot ablehnt und sich nicht in die Familienplanung eingreifen lässt? «Niemand garantiert, dass solche Frauen den begehrten Arbeitsplatz auch erhalten, wenn sie sich weigern, ihre Schwangerschaft den Unternehmensinteressen anzupassen», sagt Frank Mathwig.» (Nordwestschweiz, 2014)

Kritisch beurteilt wird ebenfalls, dass Social Freezing den Fokus der Debatte sozialer Probleme auf die weibliche Biologie lenkt anstatt auf gesellschaftliche Strukturen und Arbeitsbedingungen. Gleichzeitig wird die Verantwortung für die Familienplanung allein der Frau zugesprochen und werden Männer nur als «Statisten» wahrgenommen.

"So wie die Antibabypille die Verhütung ein für alle Mal an die Frauen delegiert hat, wird das social freezing ihnen die alleinige Verantwortung für die Reproduktion aufbürden. Für die Emanzipation bedeutet das nicht weniger als die nächste Eiszeit: Die gesellschaftlichen Strukturen werden sich verhärten. Die Doppelmo- 
ral, dass Frauen zwar berufstätig, aber trotzdem hundert Prozent Mutter sein sollen, wird unangetastet konserviert.» (NZZ am Sonntag, 2014)

«Die Männer würden weiterhin aussen vor gelassen, womit das Social Freezing die aktuellen Verhältnisse zusätzlich zementiere (N. Althaus - Journalistin). [...] Noch weiter geht die Theologin und Medizinethikerin Ruth Baumann-Hölzle, Leiterin des Instituts Dialog Ethik in Zürich: ‘Mit dem Social Freezing bleibt wieder alles an der Frau hängen. Und damit erreicht man das Gegenteil von Emanzipation.ı» (SonntagsZeitung, 2015)

«Durch den Fokus auf die Eizelle wird die Frage der Familienplanung wieder allein mit den Karrierewünschen der Frauen verknüpft. Dass die Familienplanung auch Sache der Männer ist, rückt in den Hintergrund.» (Jusletter, 2015, C. Peter - Jurist)

«Das ist perfide Biopolitik. Früher oder später können sich Frauen nur noch für Jobs bewerben, wenn sie den Nachweis erbringen, dass sie ihre Eier eingefroren haben. Ich stelle mir vor, wie eine junge Frau ihrem Chef sagen muss, dass sie jetzt ein Kind will und auf den finanziellen Zustupf fürs Einfrieren verzichtet. Zudem ist der Vorschlag sexistisch: Auch Männer werden Väter. Oder hab ich da was verpasst?» (Blick, 2014, R. Stämpfli - Politologin)

Nur selten werden auch positive Beiträge zum Social Freezing für die Frau erwähnt und die Technik als komplementär zu anderen Fördermassnahmen für bessere Chancengleichheit und höhere Vereinbarkeit von Beruf und Familie präsentiert.

"Gerade in der Anfangsphase einer Karriere, wo nun mal Leistung zählt, Flexibilität und überdurchschnittlicher Einsatz, sind Frauen um die 30 dem Druck ausgesetzt, sich bald für Kinder oder Karriere entscheiden zu müssen. Indem diese Methode die Endgültigkeit der Entscheidung fünf bis zehn Jahre auf Eis legt, nimmt sie den Druck und das schlechte Gewissen ein bisschen von uns. Sie bietet ein Stück weit Chancengleichheit und auch Komfort; während der Zeit kann man sich auf die Karriere konzentrieren, den passenden Partner zur Familiengründung finden oder eben, seinen Lebensentwurf entspannt ergründen.» (Basler Zeitung, 2014, T. Wernli - Journalistin) 
"La congélation d'ovocytes permettrait de «relâcher la pression» qui pèse sur les femmes souhaitant mener une carrière et avoir des enfants.» (Le Matin, 2014)

Die Frage nach dem Einfluss des Social Freezing auf Familienbilder findet keine grosse Erwähnung in den Medien. In einem Artikel wird darauf hingewiesen, dass aufgrund grösserer Altersunterschiede zwischen den Eltern und ihren Kindern die Generation der Grosseltern verschwinden könnte und damit eines der wenigen positiv besetzten Altersbilder.

\subsection{Diskussion der finanziellen Dimension des Social Freezing in den Schweizer Medien}

Die Kosten des Social Freezing für Stimulation, Einfrieren und Lagerung sind beinahe in allen Artikeln ein Thema. Häufig werden detaillierte Kostenschätzungen aufgeführt. Fast immer wird Social Freezing dabei als eher kostspielige Technik beschrieben, gerade weil für eine Schwangerschaft meist mehrere Zyklen notwendig seien. Daher, so das Fazit, würden sich bislang nur finanziell gut gestellte Frauen das Verfahren leisten können. In einem Leserkommentar wird dann auch kritisch von "Geldmacherei» der Reproduktionskliniken gesprochen. Es wird auch auf die Problematik der «falschen Sicherheit» verwiesen, die den Frauen in der Werbung kommuniziert werde. In mehreren Artikeln wird an die Adresse von Privatkliniken und Kinderwunschzentren appelliert, dass diese keine falschen Hoffnungen schüren sollten und Social Freezing nicht als «Versicherung gegen Kinderlosigkeit» angepriesen werden dürfe.

In einigen wenigen Artikeln wird die Thematik der Nichtbeteiligung der Krankenkassen an den Kosten für die Befruchtung in vitro und folglich am Social Freezing diskutiert. Es werden Bedenken dazu geäussert, dass die Anzahl Frauen, für die Social Freezing infrage kommt, über die hohen Kosten eingeschränkt werde. Dies könne eine soziale Diskriminierung von schlechter Verdienenden begünstigen.

"Die hohen Kosten für Social Freezing erzeugen offensichtlich ein gesellschaftliches Ungleichgewicht. Oft kommen nur besser verdienende Frauen zu uns.» (SonntagsBlick, 2014, M. von Wolff - Reproduktionsmediziner) 
«Ein Euphemismus (‘Social Freezing»), denn sozial ist an der Methode nichts. Sie ist für viele Frauen unerschwinglich. Zu den hohen Kosten für die künstliche Befruchtung kommen die Kosten für die Lagerung der Eizellen hinzu.» (NZZ am Sonntag, 2014)

«Es ist ein zynisches Argument, dass Paare Mitverantwortung für ihre Unfruchtbarkeit tragen und deshalb Kosten selber übernehmen sollen, strittig was «Eigenverantwortung> im Gesundheitsbereich heisst.» (Annabelle, 2015, B. Bleisch Philosophin)

In mehreren Artikeln, welche sich kritisch in Bezug auf Social Freezing äussern, wird auf die finanziellen Kosten hingewiesen, die beim Eintreten von medizinischen Risiken infolge des Social Freezing entstehen und schliesslich von der Allgemeinheit getragen werden müssten.

«Mehr Komplikationen und höhere Kosten für die Allgemeinheit sind programmiert.» (Neue Luzerner Zeitung, 2013, T. Eggimann - Reproduktionsmediziner)

"Jede Haftpflichtversicherung von Geburtshelfern kann en détail erklären, was es kostet (knapp 20 Millionen auf die Lebenszeit aufgerechnet), wenn bei einer Geburt (notabene haben wir es mit Risikoschwangerschaften zu tun) etwas «schiefläufts. Zudem wissen wir Mediziner seit neuestem, dass Frühgeburten (auch hiermit muss vermehrt gerechnet werden) ein erhöhtes kardiovaskuläres Risiko in späteren Lebensjahren aufweisen. Darüber hinaus gilt es zu beachten, dass pensionierte Eltern bezüglich Ausbildungskosten ihrer Kinder ebenfalls an den Staat gelangen können (was derzeit ja bereits zu Diskussionen führt). Unter dem Strich wird die oben erwähnte Rechnung insbesondere durch diejenigen verheirateten Paare bezahlt, welche mit Blick auf die Karriere bewusst auf Kinder verzichtet oder mit Blick auf den Kinderwunsch auf einen Teilschritt in ihrer beruflichen Karriere verzichtet haben.» (Briefe an die NZZ, 2014, T. Schweri - Reproduktionsmediziner)

Ob Unternehmen in der Schweiz sich überhaupt eine Kostenbeteiligung vorstellen könnten, wird in den Medien nicht thematisiert. Angesichts der Tatsache, dass die Kostenbeteiligung durch Facebook und Apple in den Schweizer Medien sehr kritisch beurteilt wird, scheint dies kaum befürwortet zu werden. 


\subsection{Diskussion der rechtlichen Dimension des Social Freezing in den Schweizer Medien}

Die Mediendiskussion zu den rechtlichen Rahmenbedingungen des Social Freezing in der Schweiz dreht sich primär um die begrenzte Aufbewahrungsfrist von maximal fünf Jahren. Dieses Thema wird in zwölf Artikeln behandelt. Dies führe dazu, dass eine Mehrheit der Frauen, die Social Freezing durchführen möchten, ins Ausland reisen würde. In einigen Artikeln wird die politische Absicht diskutiert, die Frist auf zehn Jahre zu verlängern. Zitierte Expertinnen und Experten sind sich einig, dass die Verlängerung der Aufbewahrungsfrist die Anwendung des Social Freezing stark erleichtern würde. Eine Lagerfrist von zehn Jahren wird für den Grossteil der Frauen als ausreichend beurteilt.

«LDiese Ausweitung [der Aufbewahrungsfrist auf zehn Jahre] macht einen wesentlichen Unterschied,, sagt Michael von Wolff, Präsident der FertisaveKommission der Schweizerischen Gesellschaft für Reproduktionsmedizin, welche sich um Fragen und Probleme der sogenannten fruchtbarkeitserhaltenden Massnahmen kümmert. Auch Dorothea Wunder, Reproduktionsmedizinerin in Lausanne, glaubt, ‘dass diese Verlängerung die Anwendung des Social Freezing stark erleichtert, weil man dann mehr Zeit hats.» (Tages-Anzeiger, 2016)

In wenigen Artikeln wird die Meinung erläutert, dass die Frist zur Einfrierung der Eizellen sich nicht an einer befristeten Zeitdauer, sondern an dem Lebensalter der Mutter orientieren sollte.

«Für die Reproduktionsmediziner ist das aber der falsche Ansatz. «In jedem Fall wäre es besser, nicht nach der Zeitdauer, sondern nach dem Lebensalter einzufrieren,, sagt Bruno Imthurn. Er schlägt deshalb vor, dass anstelle von fünf Jahren Eizellen bis beispielsweise zum 45. Altersjahr eingefroren werden dürfen. So liessen sich Eizellen dann entnehmen, wenn sie noch am fruchtbarsten sind, und Mütter im Grosselternalter dennoch verhindern.» (Mittelland Zeitung, 2013)

In Bezug auf die rechtliche Dimension des Social Freezing wird in mehreren Medienartikeln die Frage nach einer gesetzlich zu regelnden Alterslimite aufgeworfen. Die zitierten Reproduktionsmedizinerinnen und -mediziner wie auch Politikerinnen und Politiker sind sich häufig einig darin, dass eine solche Begrenzung sowohl aus medizinischer wie auch ethischer Sicht sinnvoll sei. Zu- 
dem würde sie die Akzeptanz für die Eizellenvorsorge in der Gesellschaft erhöhen. Die in den Artikeln präsentierten Vorstellungen einer solchen Limite reichen von maximal 45 bis zu 50 Jahren. Eine einzelne Meinung wird zitiert, die eine rechtlich festgelegte Alterslimite als "willkürlich» bezeichnet. Sinnvoller seien ärztliche Richtlinien, «welche festlegen, was Ärzte aus Sicht ihrer Profession noch als sinnvoll erachten».

«Auch Christian De Geyter, Präsident der Schweizerischen Gesellschaft für Reproduktionsmedizin, sagt: «Es gibt bei über 45-jährigen Frauen zu viele Komplikationen in der Schwangerschaft. Deshalb plädieren wir beim Social Freezing für eine Altersgrenze von 45 Jahren.) Derzeit haben sich die Schweizer Reproduktionsmediziner auf eine freiwillige Obergrenze von 50 Jahren geeinigt. Ob sie in allen Fällen eingehalten wird, ist offen. Ähnlich argumentiert Nationalrätin Verena Herzog (SVP, Thurgau). 45 Jahre seien das Maximum.» (Neue Luzerner Zeitung, 2014)

In einzelnen Artikeln wird zudem die Meinung vertreten, dass das Einfrieren von Eizellen aus sozialen Gründen nur dann rechtlich zulässig sein dürfte, wenn die Informationen und die Werbung zum Social Freezing nicht irreführend seien. Dies umfasse insbesondere, dass gesetzlich geltende Bestimmungen in der Schweiz prominent und deutlich erwähnt werden und die Betroffenen über realistische Chancen, Risiken und Kosten aufgeklärt werden müssen. Inhalt der Beratung sollte insbesondere auch der ideale Zeitpunkt des späteren Transfers der Eizelle sein. Damit könne verhindert werden, dass Institute ihre Angebote an verzweifelte, kinderlose Frauen richten, die über 35 Jahre alt sind.

Kaum ein Thema in den untersuchten Medienartikeln sind andere Voraussetzungen für IVF/ICSI wie die medizinische Indikation von Unfruchtbarkeit sowie der Umstand, dass diese Techniken heterosexuellen Paaren vorbehalten sind. 


\section{Meinungstypen zum Social Free- zing bei Schweizer Akteuren}

Dieses Kapitel widmet sich den Meinungen zum Social Freezing, welche bei politisch und gesellschaftlich relevanten Akteuren in der Schweiz existieren. Dafür wurde ein qualitativer Ansatz gewählt, der sich bei der Interpretation der Ergebnisse quantitativer Methoden zum Clustering der Antworten bedient (QMethode). Im Zentrum der Untersuchung stand die Identifizierung von Meinungstypen zum Social Freezing sowie zu den zwischen den Meinungstypen bestehenden Unterschieden und Gemeinsamkeiten. Die Ergebnisse widerspiegeln subjektive Meinungen und Einschätzungen zum Thema und beruhen nicht auf einer repräsentativen Umfrage. Die Häufigkeit dieser Meinungstypen und ihre Verteilung in der Bevölkerung sind daher unbekannt.

Für die vorliegende Studie wurden 23 Personen zum Social Freezing befragt, darunter Vertretende nationaler politischer Parteien sowie Vertretende von gesellschaftlichen Gruppierungen wie Kirchenvertretende und Fachorganisationen (siehe Liste der Interviewpartner in Anhang A3). Den interviewten Personen wurden 51 Statements mit jeweils unterschiedlichen Aussagen zum Social Freezing zur Bewertung vorgelegt. Die Statements wurden so ausgewählt, dass sie die Diskussionsthemen zum Social Freezing aus ethischer, gesellschaftlicher, rechtlicher und finanzieller Hinsicht möglichst breit abdecken (siehe Liste mit Statements in Tabelle $6 \mathrm{im}$ Anhang). Die Befragten mussten angeben, wie sehr sie die Statements befürworten beziehungsweise ablehnen ( +5 bis -5$)$.

Nachfolgend werden in einem ersten Teil die identifizierten Meinungstypen beschrieben. In einem zweiten Teil werden Gemeinsamkeiten und Unterschiede in den Meinungsbildern der befragten Akteure sowie wichtige Fragestellungen des politischen Meinungsdiskurses zum Social Freezing in der Schweiz thematisiert. 


\subsection{Beschreibung der drei Meinungstypen zum Social Freezing}

Durch das Clustering der Antworten der 23 befragten Akteure konnten drei Meinungstypen identifiziert werden. Bei den beschriebenen Meinungstypen handelt es sich um Idealtypen, die nicht vollständig mit den realen Meinungsbildern der Gesprächsteilnehmenden übereinstimmen. Jedem Meinungstyp konnten aber mindestens fünf Gesprächsteilnehmende zugeordnet werden, deren Meinung stark mit dem jeweiligen Meinungstyp korrespondiert (siehe Auflistung der Faktorenladungen in Anhang A3). Ein paar der befragten Personen können nicht klar einem Meinungsbild zugeordnet werden, sondern decken je nach Fragestellung mehrere Meinungstypen ab.

Die drei identifizierten Meinungstypen werden im Folgenden anhand ihres idealtypischen Meinungsbilds beschrieben. Als Grundlage der Beschreibung dienten erstens Statements, die sehr positiv beziehungsweise negativ beurteilt wurden. Zweitens wurden die charakteristischen Statements berücksichtigt, die den jeweiligen Meinungstyp von den anderen beiden Typen unterscheidet (in den Klammern werden jeweils die Nummer des Statements sowie die Bewertung angegeben). Eine Auflistung aller Statements sowie ihrer Faktorenwerte findet sich in Tabelle 6 im Anhang A3.

Interessant ist die Beobachtung, dass sich die Meinungen von ähnlichen gesellschaftlichen Gruppierungen, wie z.B. verschiedenen Kirchenvertretenden oder politisch nahestehenden Parteien, je nach Gesprächspartnerin und Gesprächspartner erheblich voneinander unterscheiden können. Eine Erklärung dafür ist, dass die Meinung zum Thema Social Freezing stark von persönlichen Faktoren geprägt ist, wie beispielsweise von der Geschlechterzugehörigkeit, dem Alter, der Familiensituation sowie persönlichen Erfahrungen mit Techniken der Fortpflanzungsmedizin. Da die Meinungsbildung innerhalb der Organisation zum Thema Social Freezing noch nicht sehr fortgeschritten ist, wurde bewusst davon abgesehen, die Gesprächspartnerinnen und -partner beziehungsweise die politische Partei oder gesellschaftliche Gruppierung, der sie angehören, den einzelnen Meinungstypen zuzuordnen. 


\section{Ablehnender Meinungstyp}

Der ablehnende Meinungstyp repräsentiert eine sehr kritische Haltung gegenüber Social Freezing. Zugehörige zu diesem Meinungstyp sind grundsätzlich gegen diese Technik. Ausschlaggebend sind in erster Linie individualethische Bedenken, beispielsweise was die Risiken der Anwendung des Social Freezing für das physische und psychische Kindeswohl betrifft.

Ablehnender Meinungstyp: Social Freezing als Ausdruck eines technischen Machbarkeitswahns

Hauptgrund der Skepsis gegenüber Social Freezing sind für den ablehnenden Meinungstyp die befürchteten negativen Einflüsse auf das psychische und physische Kindeswohl. Bei Techniken wie Social Freezing bestehe die Gefahr, dass das Kindeswohl gegenüber elterlichen Wünschen in den Hintergrund trete $(E 15,+5)$. Auch würden langfristige Studien zu den Auswirkungen der Eizellkonservierung auf die Gesundheit der Kinder fehlen, weshalb die Technik nicht breit angewendet werden dürfe $(E 14,+4)$. Ebenfalls unklar sei, welchen Einfluss die Lagerung der eingefrorenen Eizellen auf die Qualität der Eizellen habe. Kritisiert wird, dass in der heutigen Gesellschaft immer mehr Risiken zur Erfüllung des Kinderwunsches in Kauf genommen würden und dabei das Wohl der Kinder vergessen werde. Diese Technik sei Ausdruck eines technischen Machbarkeitswahns des Menschen, der seine gesamte Lebensgestaltung planen und kontrollieren möchte. Wenn eine Frau zu einem gewissen Zeitpunkt keine Kinder mehr bekommen könne, habe das seine Gründe. Man solle sich der Natur nicht immer so widersetzen $(E 25,+3)$.

Sehr wichtig scheint für den ablehnenden Meinungstyp die Information der Frauen: Nur wenn Frauen alle Pro und Kontras des Social Freezing kennen, können sie ihre informierte Einwilligung erteilen. Information wird als zentrale Voraussetzung der Ausübung der Fortpflanzungsfreiheit gesehen $(E 8,+5)$. Zudem sollen Eizellen ohne medizinischen Grund nur dann eingefroren werden dürfen, wenn die Information und die Werbung durch Reproduktionskliniken nicht irreführend sind (E10, +3). Hier zeigt sich die Befürchtung, dass sich junge Frauen durch falsche Versprechungen von Reproduktionskliniken in falscher Sicherheit wiegen und die Familienplanung aufschieben könnten $(E 10,+3)$. Der ablehnende Meinungstyp sieht in der Technik Social Freezing auch keinen Fortschritt zur Befreiung der Frau oder Förderung ihrer Unabhängigkeit von der aktuellen beruflichen oder partnerschaftlichen Situation (G26, -4). Vielmehr sprechen Vertretende dieses Meinungstyps von einer «Scheinfreiheit» der Frauen bezüglich der Fortpflanzung, da sie vonseiten der Reproduktionskliniken wie auch von gesellschaftlichen Zwängen beeinflusst würden. 
Zu spezifischen Themen vertritt der ablehnende Meinungstyp die folgenden Einstellungen:

- Spende überzähliger Eizellen: Personen mit diesem Meinungstyp sind gegen die Spende von überzähligen Eizellen an unfruchtbare Frauen gegen eine Kostenbeteiligung (E2, -4). Dies führe zu einer ethisch bedenklichen Kommerzialisierung der Eizellen $(E 3,+4)$. Auch äussert der ablehnende Meinungstyp gewisse ethische Bedenken bei der Spende überzähliger Eizellen an die Forschung $(\mathrm{E} 4,-2)$.

- Altersgrenze: Aufgrund der grösseren Risiken, die bei Schwangerschaften in fortgeschrittenem Alter für Mutter und Kind bestehen, befürwortet dieser Meinungstyp die Festlegung einer Altersgrenze bei der künstlichen Befruchtung $(E 16,+4)$. Nicht befürwortet wird eine individuelle Beurteilung der Altersgrenze durch Ärztinnen und Ärzte, da dies willkürlich sei (E17, -3).

- Kostenbeteiligung durch die Krankenkassen: Da Unfruchtbarkeit im Alter für den ablehnenden Meinungstyp keine Krankheit ist, wird Social Freezing auch nicht als medizinische Präventionsmassnahme interpretiert (G33, -3). Folglich sollen sich die Krankenkassen nicht an den Kosten für die künstliche Befruchtung oder für die ovarielle Stimulation, die Eizellentnahme und die Kryokonservierung beteiligen (F42, -5 ; F44, -2).

- Rechtliche Rahmenbedingungen: Für den ablehnenden Meinungstyp sind die rechtlichen Rahmenbedingungen in der Schweiz keinesfalls zu restriktiv (R51, -5). Befürwortet wird weder eine Verlängerung der Aufbewahrungsfrist $(R 47,-3)$ noch eine Erweiterung des Zugangs zur künstlichen Befruchtung für alleinstehende Mütter oder homosexuelle Paare (R49, -1 ; $\mathrm{R} 50,-1)$.

\section{Ambivalenter Meinungstyp}

Der zweite Meinungstyp ist gespalten, was seine Einstellung gegenüber Social Freezing betrifft. Grundsätzlich steht der ambivalente Meinungstyp der Anwendung des Social Freezing im Einzelfall offen gegenüber. Eine staatliche Einschränkung der Freiheit der Frauen, sich für eine Technik wie Social Freezing zu entscheiden, wird nicht befürwortet. Dennoch äussert auch dieser Meinungstyp einige Bedenken gegenüber Social Freezing. Im Vordergrund der Kritik stehen im Gegensatz zum ablehnenden Meinungstyp nicht ethische Wertvorstellungen, sondern gesellschaftskritische Überlegungen zum Einfluss des Social Freezing auf gesellschaftliche Rahmenbedingungen. Falls sich die Technik in der Schweiz etabliert, so die Befürchtung, hätte dies negative Auswirkungen auf die Vereinbarkeit von Beruf und Familie sowie auf die Chancengleichheit der Geschlechter. 
Ambivalenter Meinungstyp: Social Freezing als Scheinlösung für gesellschaftspolitische Missstände

Für den ambivalenten Meinungstyp gibt es keine guten Gründe, einer gut informierten Frau die Anwendung des Social Freezing zu verwehren $(\mathrm{E} 12,+3)$. Eine möglichst vollständige Information wird auch hier als Voraussetzung für die Ausübung der Fortpflanzungsfreiheit gesehen (E8, +4). Dennoch sieht der ambivalente Meinungstyp hinter der Anwendung des Social Freezing nicht primär die autonome Entscheidung einer Frau, die aus der Opfer- und Verzichtsrolle heraustritt und eine verantwortungsbewusste Familienplanung praktiziert (E24, -5). Vielmehr bestehe Gefahr, dass sich junge Frauen durch das Angebot von Reproduktionskliniken in falscher Sicherheit wiegen könnten $(E 10,+3)$. Zudem wird die Inanspruchnahme des Social Freezing als Ergebnis gesellschaftlicher Zwänge interpretiert $(\mathrm{E} 11,+2)$.

Der ambivalente Meinungstyp hat Bedenken für den Fall, dass sich Social Freezing in der Gesellschaft mit breiter Anwendung etabliert. Befürchtet wird ein negativer Einfluss des Social Freezing auf die gesellschaftlichen Bemühungen zur besseren Vereinbarkeit von Beruf und Familie sowie zur Förderung der Chancengleichheit. So teilt der ambivalente Meinungstyp die Ansicht, dass die Bereitschaft von Unternehmen abnehmen könnte, die Vereinbarkeit von Beruf und Familie (z.B. durch flexiblere Arbeitszeiten oder Subventionierung von Kinderbetreuung) zu unterstützen (G29, +5). Zudem könnten Unternehmen Schwangerschaften zukünftig als «vermeidbar» ansehen. Unter diesen Bedingungen würde die Anwendung des Social Freezing zu einer sozialen Vorgabe für Frauen, die sich noch beruflich entwickeln möchten $(G 28,+4)$. Kritisiert wird in diesem Zusammenhang auch die Beteiligung des Arbeitgebers an den Kosten für Social Freezing. Dies würde junge Frauen zusätzlich unter Druck setzen, die Familiengründung zugunsten ihrer Kariere zurückzustellen (F41, +3). Entsprechend wird im Social Freezing keine Technik gesehen, welche einen Beitrag an die Unabhängigkeit der Frau leistet oder diese vom Druck erlöst, sich für Kinder oder Karriere zu entscheiden (G26, -4; G27, -4). Im Gegenteil, durch die breite Anwendung der Technik würde die Diskriminierung der Frauen noch verstärkt. Statt die gesellschaftlichen Rahmenbedingungen zu verbessern und damit partnerschaftliche Kinderbetreuung und berufliche Karriere beider Partner zu ermöglichen, werde die Verantwortung für die Vereinbarung von Beruf und Familie mit Social Freezing einmal mehr an die Frau abgeschoben (G30, +5). 
Zu spezifischen Themen vertritt der ambivalente Meinungstyp die folgenden Einstellungen:

- Spende überzähliger Eizellen: Die ökonomische Situation einer Frau sollte nicht deren Zugang zur Fortpflanzungsmedizin bestimmen. Daher wird die Spende von überzähligen Eizellen an unfruchtbare Frauen, die sich im Gegenzug an den Kosten beteiligen, grundsätzlich befürwortet $(E 2,+2)$. Eine Spende überzähliger Eizellen an die Forschung wird dabei nicht als ethisch weniger heikel beurteilt als eine Spende an eine unfruchtbare Frau $(E 5,-3)$.

- Kostenbeteiligung durch die Krankenkassen: Der ambivalente Meinungstyp äussert sich eher kritisch zu einer Beteiligung an den Kosten, sowohl bei der ovariellen Stimulation, der Eizellentnahme und der Kryokonservierung wie auch bei der künstlichen Befruchtung (F42/F43, $-1)$.

- Altersgrenze: Der ambivalente Meinungstyp ist eher kritisch eingestellt gegenüber der Festlegung einer fixen Altersgrenze. Klar abgelehnt wird das Argument, dass ältere Mütter zu wenig Energie für die Kinderbetreuung oder Kinder älterer Mütter ein höheres Verwaisungsrisiko hätten (E18, -4; E20, -3). Eine Altersgrenze wird auch nicht unbedingt als sinnvollere Alternative zur Limitierung der Aufbewahrungsfrist beurteilt (R48, -2). Argumentiert wird, dass die Altersgrenze eine nicht legitime Einschränkung der Selbstbestimmung der Frau sei. Bei Männern werde das Alter beim Kinderwunsch auch nicht hinterfragt.

- Rechtliche Rahmenbedingungen: Auf rechtlicher Ebene steht dieser Meinungstyp für eine Lockerung der rechtlichen Rahmenbedingungen ein, insbesondere was die Öffnung des Zugangs zur Fortpflanzungsmedizin für homosexuelle Paare betrifft $(\mathrm{R} 50,+4)$. Ebenfalls befürwortet werden eine Verlängerung der Aufbewahrungsfrist der gefrorenen Eizellen $(R 47,+2)$ sowie der Zugang von alleinstehenden Müttern zur künstlichen Befruchtung (R49, +2). Hier wird argumentiert, dass der Staat kein Recht darauf habe, zu werten, ob lesbische und/oder alleinstehende Frauen ihre Mutterrolle schlechter ausübten als heterosexuelle Paare.

\section{Befürwortender Meinungstyp}

Der dritte Meinungstyp präsentiert im Vergleich zu den anderen beiden Meinungstypen eine befürwortende Haltung zum Social Freezing. Im Vordergrund steht hier der Glaube an die Selbstbestimmung der Frau, die sich, falls gut informiert, selbstverantwortlich für Social Freezing entscheiden kann. Damit repräsentiert dieser Meinungstyp in vielen Punkten eine gegenteilige Meinung zu den individualethischen Bedenken des ablehnenden Meinungstyps sowie zu den gesellschaftskritischen Bedenken des ambivalenten Meinungstyps. 
Befürwortender Meinungstyp: Social Freezing als Ausdruck und Mittel der selbstbestimmten Frau

Die Einstellung dieses Meinungstyps gegenüber Social Freezing ist von der Annahme geprägt, dass keine Frau eine Entscheidung zum Social Freezing leichtfertig trifft. Dafür sei es ein zu unangenehmer Prozess ohne hundertprozentige Erfolgsgarantie $(E 13,+5)$. Im Gegensatz zu den anderen Meinungstypen steht hier die Überzeugung im Vordergrund, dass die Inanspruchnahme des Social Freezing nicht Ausdruck gesellschaftlicher Zwänge ist, sondern auf einem informierten und autonomen Entscheid der Frau basiert $(E 11,+5)$. Eine entsprechende Information der Frauen ist dabei eine wichtige Voraussetzung $(E 8,+4)$. Betont wird auch eine gewisse Selbstverantwortung der Frau, sich eigenständig zu informieren. Das Argument, Frauen müssten vor den ökonomischen Interessen von Anbietern des Social Freezing geschützt werden, wird als sexistisch und bevormundend beurteilt $(\mathrm{F} 40,+3)$.

Dieser Meinungstyp sieht im Social Freezing eine sinnvolle Alternative zur Eizellspende, weil die Frau sich selbst dem aufwendigen Verfahren der Eizellentnahme unterzieht, und nicht eine fremde und unbeteiligte Eizellspenderin $(E 6,+4)$, und weil beim Social Freezing eine genetische Verbindung zwischen Mutter und Kind bestehen bleibt $(E 7,+5)$. Überhaupt nicht geteilt werden die Befürchtungen von Meinungstyp A, dass bei Techniken wie Social Freezing das Kindeswohl gegenüber elterlichen Wünschen in den Hintergrund tritt $(E 15,-4)$. Für diesen Meinungstyp ist die Anwendung von medizinischen Lösungen für soziale und gesellschaftliche Probleme wie fehlender Partner, Unvereinbarkeit von Karriere und Kindern durchaus berechtigt (G32, -5$)$.

Im Gegensatz zum ambivalenten Meinungstyp dominiert bei diesem Meinungstyp die Überzeugung, dass Social Freezing keinen negativen Einfluss auf die gesellschaftlichen Bemühungen zur Vereinbarkeit von Beruf und Familie hat. Es wird keine Gefahr darin gesehen, dass Unternehmen die Vereinbarkeit von Beruf und Familie zukünftig weniger unterstützen könnten oder Social Freezing zu einer sozialen Vorgabe für berufstätige Frauen werden könnte (G28, -2; G29, -2). Auch die Kostenbeteiligung durch Unternehmen setzt die Frauen nicht zwingendermassen unter Druck (F40, -2$)$. Ebenfalls nicht geteilt wird die Befürchtung, dass Social Freezing zu einer Verstärkung der Diskriminierung der Frauen führt $(\mathrm{G} 30,-3)$. Der befürwortende Meinungstyp geht davon aus, dass sich die technologische Entwicklung nach einem tatsächlich bestehenden Bedarf in der Gesellschaft orientiert. Die Nachfrage nach Social Freezing werde nicht durch Anbieter der Technologie künstlich gesteigert, sondern die Technik entspreche in der Regel den Bedürfnissen der Frauen (F39, -4). 
Zu spezifischen Themen vertritt der befürwortende Meinungstyp die folgenden Einstellungen:

- Spende überzähliger Eizellen: Einer Spende von überzähligen Eizellen an unfruchtbare Frauen gegen Beteiligung an den Kosten sowie an die Forschung steht der befürwortende Meinungstyp grundsätzlich offen gegenüber (E2, +2; E5, +1).

- $\quad$ Altersgrenze: Keine Zustimmung findet das Argument, dass eine Altersgrenze notwendig sei, um zu verhindern, dass ein Kind früh seine Mutter verliert (E20, -3). Im Gegenteil, dank der höheren Lebenserwartung könne eine Frau heute problemlos später Mutter werden und ihr Kind bis zur Volljährigkeit begleiten $(E 21,+3)$. Eine Beschränkung der künstlichen Befruchtung wird ab dem Alter als sinnvoll erachtet, ab dem eine Schwangerschaft mit grösseren Risiken für Mutter und Kind verbunden ist $(\mathrm{E} 16,+2)$.

- Kostenbeteiligung durch die Krankenkassen: Der befürwortende Meinungstyp befürwortet die Beteiligung der Krankenkassen an den Kosten für die künstliche Befruchtung (F42, +4). Eher nicht befürwortet wird die Beteiligung der Krankenkassen an den Kosten der ovariellen Stimulation, Eizellentnahme und Kryokonservierung, da ungewiss ist, ob die eingefrorenen Eizellen überhaupt je gebraucht werden $(\mathrm{F} 43,+1)$.

- Rechtliche Rahmenbedingungen: Bezüglich der rechtlichen Rahmenbedingungen würde der befürwortende Meinungstyp eine Verlängerung der Aufbewahrungsfrist der eingefrorenen Eizellen als sinnvoll erachten $(\mathrm{R} 47,+3)$. Auch der Lockerung von Rahmenbedingungen bezüglich des Zugangs alleinstehender Mütter $(R 48,+2)$ sowie homosexueller Paare (R49, +1) zur künstlichen Befruchtung steht er offen gegenüber.

\subsection{Konsens und Kompromisse im politischen Meinungsdiskurs}

In diesem Kapitel werden die Gemeinsamkeiten und Unterschiede zwischen den Meinungstypen thematisiert. Daraus können einerseits diejenigen Themen abgeleitet werden, bei denen ein Konsens besteht. Andererseits werden diejenigen Themen sichtbar, bei denen keine Einigkeit besteht und bei denen in einem allfälligen politischen Diskurs Kompromisse gesucht werden müssten. 


\section{Informationspflicht als Konsens}

Die einzige Aussage, die von allen drei Meinungstypen eine klare Zustimmung erhält, lautet: «Nur, wenn Frauen alle Pro und Kontras zum Social Freezing kennen, können sie ihre informierte Einwilligung erteilen. Ein informierter Entscheid ist eine wichtige Voraussetzung für die Ausübung der Fortpflanzungsfreiheit»(E8). Der ablehnende sowie der ambivalente Meinungstyp erachten Information vor allem deshalb als wichtig, weil Frauen sich aufgrund des Angebots oder auch irreführender Werbung von Anbietern des Social Freezing in falscher Sicherheit wiegen könnten. Der befürwortende Meinungstyp hingegen vertritt nicht die Meinung, dass Frauen vor den ökonomischen Interessen der Anbieter der Technik geschützt werden müssen. Die Information wird aber auch hier als eine wichtige Voraussetzung für eine selbstbestimmte, autonome Entscheidung angesehen. Die Zustimmung zu einer gesetzlich geregelten Informationspflicht scheint bei einigen Personen von der Regelungsdichte, z.B. zu Art und Umfang der Information abzuhängen. Einige kritische Stimmen wiesen zudem darauf hin, dass ein «informed consent» nur ein soziales Konstrukt sei, da neutrales und vollständiges Wissen nicht existiere. Dass jedoch ein Minimum an Informationen garantiert werden sollte, scheint unbestritten. Entsprechend könnte die gesetzliche Informationspflicht in einer zukünftigen politischen Debatte forciert werden, ohne auf grossen politischen Widerstand zu stossen.

\section{Aufbewahrungsfrist oder Altersgrenze}

Soll die heutige Aufbewahrungsfrist verlängert werden? Zu dieser Frage herrscht Uneinigkeit. Zustimmung erhält der Vorschlag zur Verlängerung der Aufbewahrungsfrist vom ambivalenten und vom befürwortenden Meinungstyp. Der ablehnende Meinungstyp äussert sich klar gegen eine solche Verlängerung (R47), wohl deshalb, weil man sich generell keine Lockerung der rechtlichen Rahmenbedingungen für Social Freezing wünscht. Zudem sind Bedenken dazu vorhanden, dass die Qualität der Eizellen bei längerer Aufbewahrungsdauer abnehmen könnte. Bei einer Verlängerung der Aufbewahrungsfrist wäre in einer politischen Debatte mit Widerstand zu rechnen. In den Gesprächen wird zudem die Meinung vertreten, dass eine Aufbewahrungsfrist, wie sie heute existiert, nicht rechtlich durchsetzbar ist, da der Staat keine Eizellen ohne Einwilligung der Eltern vernichten könne. 
Soll anstelle einer Aufbewahrungsfrist eine Altersgrenze eingeführt werden? Die Analyse zeigt, dass bezüglich einer Altersgrenze unterschiedliche Meinungen vorhanden sind. Der ablehnende und der befürwortende Meinungstyp sprechen sich für eine Altersgrenze aus, und zwar primär aufgrund grösserer Risiken für Mutter und Kind bei Schwangerschaften im fortgeschrittenen Alter (E16). Der ambivalente Meinungstyp ist eher gegen eine fixe Altersgrenze (E16, R48). Von einigen Personen wird in diesem Zusammenhang diskutiert, dass eine Altersgrenze auch für Männer gelten müsste. Schliesslich stelle das Alter auch bei Vätern einen nicht zu vernachlässigenden medizinischen Risikofaktor dar. Die Statements zur Altersgrenze für Männer erhielten jedoch keine hohen Bewertungen und scheinen entsprechend nicht so zentral zu sein (E22, 23). Eine Altersgrenze, welche auf einer individuellen Risikobeurteilung durch Ärztinnen und Ärzte basiert, findet nur beim ambivalenten Meinungstyp eine Zustimmung. Klar abgelehnt wird dies durch den ablehnenden Meinungstyp, während der befürwortende Meinungstyp hier unentschieden scheint (E17).

Zusammenfassend lässt sich feststellen, dass es sich bei «Altersgrenze statt Aufbewahrungsfrist» um eine Frage handelt, bei der die Meinungsfindung nicht eindeutig ist beziehungsweise noch nicht abgeschlossen zu sein scheint. Die grosse Mehrheit der befragten Akteure wünscht sich eine Altersbeschränkung bei der In-vitro-Befruchtung, insbesondere gestützt auf medizinische Kriterien. Eine Altersgrenze aus sozialen Argumenten, wie beispielsweise, dass ältere Mütter zu wenig Energie haben oder zu wenig Unterstützung durch Grosseltern erhalten, wird eher abgelehnt. Bei der Frage, was Vor- und Nachteile einer Altersgrenze gegenüber der heutigen Aufbewahrungsfrist sind, scheint noch Klärungsbedarf zu bestehen.

Spende von überzähligen Eizellen an unfruchtbare Frauen gegen Kostenbeteiligung?

Gemäss dem ambivalenten und dem befürwortenden Meinungstyp sollte eine Spende an unfruchtbare Frauen, die sich im Gegenzug an den Kosten beteiligen, möglich gemacht werden (E2), insbesondere aus dem Grund, um die soziale Ungleichheit von gut und weniger gut verdienenden Frauen zu reduzieren. Der ablehnende Meinungstyp spricht sich klar dagegen aus, da eine Kommerzialisierung der Eizellen befürchtet wird (E3). Mehrere Gesprächspartnerinnen und -partner betonen in diesem Zusammenhang, dass sie die Kostenbeteili- 
gung grundsätzlich befürworten, jedoch nur bei einer Beteiligung an den tatsächlichen medizinischen Kosten und nicht im Sinne einer darüber hinausgehenden finanziellen Entgeltung, die dann tatsächlich zu einer Kommerzialisierung führen könnte. Bei einer zukünftigen politischen Debatte über dieses Thema würde wohl die Höhe der Kostenbeteiligung einen entscheidenden Faktor darstellen.

\section{Kostenbeteiligung durch die Krankenkassen?}

Die finanzielle Beteiligung der Krankenkassen an den Kosten für die ovarielle Stimulation, die Eizellentnahme sowie die Lagerung der Eizellen scheint für die Akteure nicht so umstritten zu sein (F43). Die Mehrheit der Befragten ist der Ansicht, dass die Kosten eher von der Frau selbst übernommen werden sollten. Bei der Frage nach einer Beteiligung der Krankenkassen an den Kosten für die In-vitro-Befruchtung herrscht grössere Uneinigkeit (F42). Eine Mehrheit äussert sich eher kritisch gegenüber einer solchen Kostenbeteiligung. Der ablehnende Meinungstyp ist klar gegen die Kostenbeteiligung, der ambivalente Meinungstyp ist ebenfalls eher kritisch eingestellt (F42). Der befürwortende Meinungstyp spricht sich als einziger für eine Kostenbeteiligung aus. Diejenigen Personen, die sich eine finanzielle Beteiligung durch die Krankenkassen wünschen, machen dies mehrheitlich abhängig vom Alter der Frau - den Kinderwunsch einer Frau in einem Alter deutlich über der biologischen Fruchtbarkeitsgrenze will man nicht mitfinanzieren. Hier gelte es, genauer zu klären, bis in welches Alter die ungewollte Kinderlosigkeit beziehungsweise Unfruchtbarkeit als Krankheit definiert werden kann.

Weitere Punkte, die in einer allfälligen politischen Debatte über Social Freezing diskutiert würden, sind Fragen nach den Risiken für das physische und psychische Kindeswohl und welche empirische Evidenz diesbezüglich besteht. Zudem wird auch immer die Frage nach dem Einfluss des Social Freezing auf gesellschaftliche Rahmenbedingungen zur Vereinbarkeit von Beruf und Familie sowie zur Förderung der Chancengleichheit eine Rolle spielen. Die Relevanz dieser Argumentation ist jedoch stark davon abhängig, wie das Potenzial von SocialFreezing-Anwendungen in der Schweiz eingeschätzt wird. Wenn davon ausgegangen wird, dass Social Freezing in der Gesellschaft eine Einzelfallösung bleibt, erhält die gesellschaftliche Kritik am Social Freezing weniger Bedeutung. 


\section{Rechtsvergleichung}

\section{1 Übersicht}

Nachfolgend werden zunächst die aktuelle Rechtslage in der Schweiz sowie anschliessend aus rechtsvergleichender Perspektive die Rechtslage in Österreich, Frankreich, Italien, Deutschland, Belgien, Spanien und in der Tschechischen Republik dargestellt.

In der Schweiz ist Social Freezing nicht explizit geregelt. In Österreich ist Social Freezing unzulässig, Frankreich kennt eine sehr restriktive Regelung. In Italien ist die Rechtslage insbesondere in Bezug auf die Altersgrenze bei der Frau nicht vollständig geklärt. Rechtsunsicherheiten bestehen ferner in Deutschland, das überhaupt kein Fortpflanzungsmedizingesetz hat. Sehr grosszügige Regelungen zum Social Freezing finden sich hingegen in Belgien, Spanien sowie in der Tschechischen Republik, wobei insbesondere die letzteren beiden Länder sich mit ihrem fortpflanzungsmedizinischen Angebot explizit an Medizintouristen wenden (siehe auch Kapitel 9.1.6 für eine Übersicht).

\subsection{Schweiz}

In der Schweiz ist Social Freezing nicht explizit geregelt. Insbesondere wird die Eizellenvorsorge im Bundesgesetz über die medizinisch unterstützte Fortpflanzung (FMedG) vom 18. Dezember 1998 mit den seither in Kraft getretenen Revisionen nicht ausdrücklich erwähnt.

In der Literatur geht man davon aus, dass der erste Schritt der Eizellenvorsorge, nämlich die Entnahme der Eizellen, nicht dem FMedG unterliegt und daher ohne medizinische Indikation zulässig ist. Dies ergibt sich aus der Definition des Fortpflanzungsmedizinverfahrens in Art. 2 Bst. a FMedG, wonach darunter eine «Methode zur Herbeiführung einer Schwangerschaft ohne Geschlechtsverkehr» zu verstehen ist. Bei der Entnahme und Kryokonservierung der Eizellen geht es 
aber gerade noch nicht um die Herbeiführung einer Schwangerschaft. Entsprechend gelten die fortpflanzungsmedizinischen Grundsätze, Informations- und Beratungspflichten des FMedG nicht. Bei der Behandlung dürfte es sich jedoch, wie bei einem normalen Arztvertrag, um einen Auftrag im Sinne des Obligationenrechts handeln, sodass die in Lehre und Rechtsprechung herausgearbeiteten Aufklärungs- und Sorgfaltspflichten des Arztes ohne Weiteres zur Anwendung gelangen.

Für die zweite Phase der Eizellenvorsorge, nämlich die Kryokonservierung, findet sich im FMedG hingegen eine konkrete Regelung: Die Höchstdauer der Aufbewahrung beträgt nämlich nach Art. 15 Abs. 1 FMedG fünf Jahre. Diese Frist kann nach geltendem Recht einmalig und ohne nähere Begründung um weitere fünf Jahre verlängert werden. Damit beträgt die maximale Aufbewahrungsdauer zehn Jahre. Dies dürfte in Zusammenhang mit Social Freezing oftmals zu kurz sein, wird doch eine möglichst frühzeitige Entnahme der Eizellen empfohlen, da Zahl und Qualität der Gameten mit zunehmendem Alter der Frau deutlich nachlassen.

Für die spätere Verwendung der Eizellen, das heisst für die In-vitro-Befruchtung und den Embryotransfer, gelten unbestritten die Bestimmungen des FMedG. Dabei ist insbesondere eine medizinische Indikation im Sinne von Art. 5 FMedG erforderlich, wozu unter anderem Unfruchtbarkeit gehört. Deren Gründe werden nicht spezifiziert, das heisst, es kann sich auch um eine altersbedingte Unfruchtbarkeit handeln. Diese Auffassung ist in der Literatur zwar nicht ganz unangefochten, mangels einer entsprechenden Klärung durch den Gesetzgeber fehlt es aber an einer Grundlage, um einer Frau, die ihre Fruchtbarkeit aus Altersgründen eingebüsst hat, den Zugang zu ihren eigenen, maximal zehn Jahre zuvor kryokonservierten Eizellen zu verwehren. Zudem dürfte in manchen Fällen ein Nachweis scheitern, dass die Unfruchtbarkeit rein altersbedingt ist, das heisst, dass die Frau zu einem früheren Zeitpunkt die Möglichkeit gehabt hätte, auf natürlichem Weg schwanger zu werden. Allerdings muss nach dem Gesetzestext bei der Anwendung fortpflanzungsmedizinischer Verfahren stets das Kindeswohl gewährleistet sein. Daraus ergibt sich auch eine «weiche» Altersgrenze, weil Fortpflanzungsverfahren nur bei Paaren angewendet werden dürfen, die aufgrund ihres Alters und ihrer persönlichen Verhältnisse voraussichtlich bis zur Volljährigkeit des Kindes für dessen Pflege und Erziehung sorgen können (Art. 3 Abs. 2 FMedG). 
Aus rein rechtlicher Sicht, das heisst ohne Rücksicht darauf, ob die Eizellenvorsorge überhaupt wünschenswert und eine Gesetzesänderung daher angebracht ist (vgl. Kapitel 9.1.6), wäre in Zusammenhang mit Social Freezing namentlich folgenden Aspekten Rechnung zu tragen:

- Eine Neuregelung in Bezug auf Social Freezing müsste sicherstellen, dass angemessene Informations- und Beratungspflichten (derzeit Art. 6 FMedG) nicht erst bei der assistierten Fortpflanzung, sondern bereits vor der hormonellen Stimulierung und Eizellentnahme bestehen, auf die derzeit, wie erwähnt, das FMedG gar keine Anwendung findet. Prüfenswert wäre eine Aufklärungs- und Beratungspflicht, die nicht nur medizinische Aspekte umfasst, sondern die sicherstellt, dass die Frau unabhängig und umfassend zu sämtlichen Aspekten der Eizellenvorsorge beraten wird. Insofern könnte sich die Einführung einer spezifischen psychologischen Beratungspflicht oder wenigstens eines entsprechenden Angebots aufdrängen.

- Für die aktuelle Aufbewahrungsdauer von maximal zehn Jahren und für die Pflicht, die Aufbewahrung nach fünf Jahren verlängern zu lassen, gibt es keine medizinischen Gründe. Nach dem aktuellen Stand von Medizin und Technik bleiben kryokonservierte Eizellen längere Zeit in gleichbleibend gutem Zustand. Je jünger die Frau zum Zeitpunkt der Eizellentnahme ist, desto besser ist die Qualität der Gameten. Die mit der maximalen Aufbewahrungsdauer verbundene Notwendigkeit, sich nach Ablauf von zehn Jahren erneut einer hormonellen Stimulation und Eizellentnahme unterziehen zu müssen, lässt sich vernünftigerweise kaum begründen. Strebt eine Frau beispielsweise im Alter von 42 Jahren eine medizinisch unterstützte Fortpflanzung an, wäre es aus medizinischen Gründen vorteilhafter, die ihr im Alter von 25 Jahren entnommenen Eizellen zu verwenden anstatt die erst zehn Jahre später entnommenen. Eine deutliche Verlängerung oder Streichung der Aufbewahrungsfrist ist daher durch den Gesetzgeber ernsthaft zu erwägen. Überlegenswert wäre es, die Vernichtung der Eizellen künftig nur noch vom Erreichen der Alters- beziehungsweise Fruchtbarkeitsgrenze (dazu sogleich) abhängig zu machen und eine Aufbewahrung bis zu diesem Zeitpunkt uneingeschränkt zu erlauben. 
- Wie erwähnt, kennt das aktuelle FMedG keine strikte Altersgrenze für die In-vitro-Befruchtung. Wird Social Freezing neu geregelt und in diesem Zusammenhang die Aufbewahrungsfrist für Eizellen, wie hier vorgeschlagen, gelockert, ist dies zu überdenken. Erst kürzlich hat der Gesetzgeber in Zusammenhang mit der Revision des Adoptionsrechts in Art. 264d Abs. 1 ZGB einen maximalen Altersunterschied zwischen Adoptivkind und Adoptiveltern(teil) von 45 Jahren eingeführt. Davon kann zwar aus Gründen des Kindeswohls im Einzelfall abgewichen werden. In Zusammenhang mit fortpflanzungsmedizinischen Verfahren ist allerdings gerade nicht auf das Wohl eines bereits lebenden, auf «neue» Eltern angewiesenen Kindes Rücksicht zu nehmen. Es ist daher nicht einzusehen, weshalb das neue Adoptionsrecht eine konkrete Altersgrenze vorsieht, das Fortpflanzungsmedizinrecht hingegen nicht. Wenn schon, müsste es aus medizinischen Gründen - nämlich mit Blick auf die Risiken von Mutter und Kind bei einer Schwangerschaft in fortgeschrittenem Alter - gerade umgekehrt sein. Der Wertungsinkongruenz zwischen Adoptions- und Fortpflanzungsmedizinrecht wäre bei einer allfälligen Regelung der Eizellenvorsorge Rechnung zu tragen.

\section{3 Österreich}

Seit 1992 existiert in Österreich ein Fortpflanzungsmedizingesetz (nachfolgend öFMedG), welches die Reproduktionsmedizin umfassend gesetzlich regelt und dabei nicht bloss einen auf Verbote ausgerichteten Ansatz verfolgt, sondern auch administrativ-prozedurale Regeln enthält.

Die Zellentnahme und -aufbewahrung ist in $\S 2 \mathrm{~b}$ öFMedG geregelt. Danach dürfen Samen, Eizellen sowie Hoden- und Eierstockgewebe nur «für eine künftige medizinisch unterstützte Fortpflanzung entnommen und aufbewahrt werden, wenn ein körperliches Leiden oder dessen dem Stand der medizinischen Wissenschaft und Erfahrung entsprechende Behandlung eine ernste Gefahr bewirkt, dass eine Schwangerschaft nicht mehr durch Geschlechtsverkehr herbeigeführt werden kann». (Abs. 1) Da im Falle der Eizellenvorsorge zum Zeitpunkt der Entnahme keine ernste Gefahr für die Fertilität infolge eines körperli- 
chen Leidens oder einer entsprechenden Behandlung gegeben ist, besteht in Österreich keine Möglichkeit, Social Freezing in Anspruch zu nehmen.

Das öFMedG wurde durch das am 21. Januar 2015 vom Nationalrat beschlossene Fortpflanzungsmedizin-Änderungsgesetz 2015 (FMedRÄG 2015) reformiert, dessen Inkrafttreten auf den 24. Februar 2015 festgesetzt war. Dem vorangegangen war eine Entscheidung des österreichischen Verfassungsgerichtshofes vom 10. Dezember 2013, der unter Berufung auf das Diskriminierungsverbot gemäss Art. 14 i.V.m. 8 der Europäischen Menschenrechtskonvention (EMRK) jene Bestimmungen aufhob, die Frauenpaare von der Inanspruchnahme der medizinisch unterstützten Fortpflanzung ausschlossen. Durch das FMedRÄG 2015 wurde jedoch nicht nur der Zugang zur Reproduktionsmedizin auf Frauenpaare ausgeweitet, sondern daneben unter anderem die Präimplantationsdiagnostik (§ 2a öFMedG) und Eizellspende erlaubt.

Es ist dabei anzumerken, dass das erwähnte Verbot der Eizellenvorsorge beziehungsweise die Unzulässigkeit der Eizellentnahme bei fehlender medizinischer Indikation bereits vor dem Erlass des FMedRÄG 2015 bestand. Der Gesetzgeber hat aber augenscheinlich trotz der mit der Reform bezweckten Liberalisierung des öFMedG darauf verzichtet, Social Freezing zuzulassen. Erstaunlich ist dabei, dass die Materialien die Fortführung des Verbots nicht begründen und auch ansonsten die Thematik des Social Freezing nicht aufgreifen trotz der Öffnung der Fortpflanzungsmedizin in Bezug auf die Eizellspende durch das FMedRÄG 2015. Im Anschluss an das Inkrafttreten des FMedRÄG 2015 ist in Österreich in der Literatur vereinzelt die EMRK-Konformität des Verbots von Social-Freezing-Anwendungen diskutiert worden.

Da Social Freezing nach dem Gesagten in Österreich unzulässig ist, gibt es auch keine diesbezüglichen Regeln zur Kryokonservierung und zum Höchstalter der Frau. In einem anderen Zusammenhang, nämlich im Kontext der Verwendung einer Eizellspende, legt das Gesetz ein Höchstalter von 45 Jahren fest ( 33 Abs. 3 öFMedG: Eine Eizellspende darf nur in Anspruch genommen werden, wenn die eigenen Eizellen der zu behandelnden Frau nicht fortpflanzungsfähig sind und die Patientin das 45 . Lebensjahr noch nicht vollendet hat). Aus $\S 17$ Abs. 1 öFMedG ergibt sich sodann, dass Eizellen maximal zehn Jahre aufbewahrt werden dürfen; von einer Verlängerungsmöglichkeit ist nicht die Rede. 


\subsection{Frankreich}

\subsubsection{Ausgangslage und Übersicht}

Die Fortpflanzungsmedizin wird in Frankreich durch das «Loi du 7 juillet 2011 relative à la bioéthique» geregelt, dessen Bestimmungen grösstenteils in den "Code de la santé publique» (nachfolgend: CSP) eingefügt wurden (vgl. zur Eizellenvorsorge insbesondere die Kapitel «Assistance médicale à la procréation» und «Don et utilisation de gamètes»). Besondere Beachtung ist zudem auch der kürzlich aktualisierten «Arrêté du 30 juin 2017 modifiant l'arrêté du 11 avril 2008 modifié relatif aux règles de bonnes pratiques cliniques et biologiques d'assistance médicale à la procréation» (nachfolgend: Guide des bonnes pratiques) zu widmen, welche die Regeln über die Techniken der medizinisch unterstützten Fortpflanzung auf Verordnungsebene umsetzt.

Das französische Recht hat in den letzten Jahren in Bezug auf die Thematik der Eizellenvorsorge («autoconservation ovocytaire») tiefgreifende Änderungen erfahren. So ist es seit Dezember 2015 unter bestimmten Voraussetzungen möglich - im Rahmen einer Eizellspende -, Social Freezing in Anspruch zu nehmen. Der Gesetzgeber wollte damit primär die Gametenspende fördern und auf diese Weise den in Frankreich vorherrschenden Mangel an Eizellen beheben: Die Möglichkeit der Eizellenvorsorge sollte dazu als «mécanisme d'incitation» - also als «Anreizmechanismus» - dienen.

Die eingeführten Neuerungen zogen kontroverse Diskussionen nach sich. Unter anderem hat sich das Comité Consultatif National d'Ethique im Juni 2017 in der Stellungnahme Nr. 126 kritisch zur neuen Möglichkeit des Social Freezing geäussert. Es hielt in seinem Bericht insbesondere fest, dass die Eizellenvorsorge auf keinen Fall als «solution magique» dargestellt werden dürfe und es wichtig sei, die Interessentinnen über die Risiken und Nebenwirkungen aufzuklären. Die sehr aktuellen Diskussionen in Frankreich sind für die Frage, ob die Rechtslage in der Schweiz revisionsbedürftig ist, von hohem Interesse. Ebenfalls von Interesse sind die sehr ausführlich geregelten Informations- und Zustimmungserfordernisse. Aus diesem Grund ist die nachfolgende Darstellung der Rechtslage in Frankreich ausführlicher ausgefallen als diejenige anderer Länder. 


\subsubsection{Zulässigkeit einer rein sozialen Indikation}

a) Grundsatz: Verbot des Social Freezing

Die medizinisch unterstützte Fortpflanzung kann nach französischem Recht dazu dienen, einerseits die Unfruchtbarkeit eines Paares zu beheben, andererseits die Übertragung einer schweren Krankheit zu verhindern (Art. L. 2142-2 Abs. 1 CSP). Mit dem Erfordernis, dass die Unfruchtbarkeit pathologischer Natur sein muss, ist nach französischem Recht allerdings die Inanspruchnahme von medizinisch unterstützter Fortpflanzung in Fällen von physiologischer Unfruchtbarkeit, worunter auch die altersbedingte Unfruchtbarkeit der Frau fällt, verboten.

Die eben erwähnten Zugangsschranken greifen bereits im Stadium der Kryokonservierung (vgl. Art. 2141-1 Abs. 1 CSP), woraus folgt, dass bereits zu diesem Zeitpunkt eine medizinische Indikation zwingend erforderlich ist. Dies ist beim Social Freezing gerade nicht der Fall, erfolgt dieses doch aus rein «sozialen» Gründen, weshalb die Entnahme und Konservierung von Eizellen zur reinen «Vorsorge» grundsätzlich unzulässig ist.

\section{b) Ausnahme: Verbindung mit Eizellspende}

Seit Dezember 2015 kann allerdings dennoch Social Freezing in Anspruch genommen werden: Im Jahr 2011 hat der französische Gesetzgeber die Neuerung eingeführt (siehe Art. L. 1244-2 Abs. 3 CSP), dass volljährige, das heisst 18jährige kinderlose Frauen, die sich für eine Eizellspende zur Verfügung stellen, einen Teil der gespendeten Gameten für die eigene spätere Verwendung im Rahmen einer künstlichen Fortpflanzung konservieren lassen können, gewissermassen als «Belohnung» für die Spende. Allerdings kann von dieser «autoconservation ovocytaire» aufgrund der stark verspäteten Publikation der dazugehörigen Durchführungsbestimmungen (Décret $n^{\circ}$ 2015-1281 du 13 octobre 2015 relatif au don de gamètes; arrêté du 24 décembre 2015 [NOR: AFSP1532457A]) erst seit Dezember 2015 auch tatsächlich Gebrauch gemacht werden. 
Die Verteilung der Eizellen bestimmt sich nach der Anzahl vorhandener reifer Eizellen, wobei der Spende gegenüber der Eigenverwendung eine Vorrangstellung eingeräumt wird (siehe Absatz V.1 Guide des bonnes pratiques):

- Beim Vorliegen von bis zu fünf reifen Eizellen: Alle Eizellen müssen gespendet werden, eine Eigenverwendung der Eizellen ist nicht möglich.

- Beim Vorliegen von sechs bis zehn reifen Eizellen: Mindestens fünf Eizellen müssen gespendet werden.

- Beim Vorliegen von mehr als zehn reifen Eizellen: Mindestens die Hälfte der Eizellen muss gespendet werden.

Der «Guide des bonnes pratiques» legt im Kapitel V.2 die medizinischen Voraussetzungen fest, um als Eizellspenderin angenommen zu werden:

- Alter von 18 bis 38 Jahren zum Zeitpunkt der Eizellentnahme;

- von einem Ärzteteam festgestellter, guter allgemeiner Gesundheitszustand sowie ein persönliches Umfeld, das mit dem Prozess der Spende vereinbar ist;

- Fehlen eines bekannten Risikos der Übertragung einer Krankheit, deren Schwere der Spende entgegensteht;

- befriedigende Ergebnisse der klinischen Untersuchung und der Infektionswerte;

- Ergebnisse der gynäkologischen Untersuchung einschliesslich einer Auswertung der Ovarialfunktion, welche gute Voraussetzungen für eine Eizellspende erwarten lassen;

- keine Kontraindikation in Bezug auf die hormonelle Stimulation und Eizellentnahme.

Sind diese Voraussetzungen nicht erfült, ist die Frau nicht zur Eizellspende zugelassen, womit auch die damit verbundene Möglichkeit des Social Freezing entfällt.

\subsubsection{Aufbewahrungsfrist}

Das französische Recht sieht keine maximale Aufbewahrungsfrist der konservierten Eizellen zum Eigengebrauch vor. In den nachfolgenden Fällen wird die 
Konservierung allerdings nicht mehr zum Zwecke des Eigengebrauchs fortgesetzt, sondern im Hinblick auf eine Spende (siehe Absatz V.1 Guide des bonnes pratiques):

- Verzicht der Spenderin auf die Konservierung der Eizellen für die spätere Eigenverwendung;

- Überschreitung des Fruchtbarkeitsalters der Spenderin (vgl. Art. L. 2141-2 Abs. 2 CSP; siehe dazu Ziff. 4);

- Ausbleiben einer Antwort auf die jährliche Kontaktaufnahme durch das Fortpflanzungsmedizinzentrum, sofern die Konservierung länger als zehn Jahre andauert;

- Tod der Spenderin.

\subsubsection{Voraussetzung für die In-vitro-Fertilisation}

Bei einer späteren Verwendung der zuvor konservierten Eizellen gelangen die allgemeinen Voraussetzungen für die medizinisch unterstützte Fortpflanzung zur Anwendung (siehe dazu vorne), was insbesondere zur Folge hat, dass eine pathologische (!) Unfruchtbarkeit vorliegen muss oder mit der assistierten Fortpflanzung die Übertragung einer schweren Krankheit verhindert werden soll (Art. L. 2142-2 Abs. 1 CSP). Ferner muss die betreffende Frau noch im Fruchtbarkeitsalter sein (Art. L. 2142-2 Abs. 2 CSP).

In der Praxis hat sich für die Frau in Bezug auf das Fruchtbarkeitsalter eine Alterslimite von zirka 42 bis 43 Jahren etabliert. Gemäss jüngster Rechtsprechung betreffend einen männlichen Patienten muss aber bei Bestimmung des Fruchtbarkeitsalters die gesamte persönliche Situation gewürdigt werden und es kann nicht einzig auf das Geburtsjahr abgestellt werden (Urteile des Tribunal Administratif Montreuil, $8^{\text {ème }}$ chambre, 14. Februar 2017, Nr. 1606644 und 1606724). 


\subsubsection{Spezifische Beratungspflichten}

Da Social Freezing nur in Zusammenhang mit einer Eizellspende zulässig ist, müssen in Bezug auf die Informationspflichten einerseits die Regelungen zur Eizellspende, andererseits aber auch die Vorschriften über die Konservierung der Eizellen zum Eigengebrauch Beachtung finden. Schliesslich stellt das Gesetz auch für eine allfällige anschliessende In-vitro-Fertilisation Regeln zur Beratung des Paares auf.

\section{a) Allgemeine Informationspflichten bei einer Eizellspende}

Die Person, die eine Eizellspende vornehmen möchte, ist in Form eines Gesprächs mit einem Mitglied des Ärzteteams über die nachfolgenden Punkte zu informieren (Kapitel V.1 Guide des bonnes pratiques):

- über die gesetzlichen und verordnungsrechtlichen Bestimmungen über die Spende (konkret: Art. L. 1244-1 ff. und R. 1244-1 ff. CSP);

- über die Pflicht, anzugeben, ob zu einem früheren Zeitpunkt eine Gametenspende oder eine Embryonenspende an ein anderes Paar erfolgt ist;

- über die Pflicht, sich einer klinisch-biologischen Untersuchung zu unterziehen, um die Durchführbarkeit einer Spende zu überprüfen, aber auch um nach allfälligen Risikofaktoren für die Spenderin selbst oder die Empfängerin der Spende zu suchen. Die Spenderin ist zudem darüber zu informieren, dass das Resultat der Untersuchungen unerwartete Defekte aufdecken kann, insbesondere in Bezug auf die Anzahl oder die Qualität der Gameten; in einer solchen Situation wird das Verfahren der Spende unterbrochen, die Anomalie wird untersucht und es können nach vollständiger Aufklärung, sofern sachdienlich, fruchtbarkeitserhaltende Massnahmen vorgeschlagen werden;

- über die Notwendigkeit, ein Gespräch mit einem Psychiater oder Psychologen zu führen;

- über die Pflicht, das Ärzteteam über einen diagnostizierten Gendefekt zu informieren, der entweder bei der Spenderin selbst oder einer verwandten Person vorliegt und geeignet ist, durch die Spende übertragen zu werden.

Nebst diesen Informationspflichten, die sowohl für männliche als auch weibliche Spender gelten, muss die Eizellspenderin zusätzlich im Rahmen des Ge- 
sprächs mit dem Ärzteteam über die Risiken und Nebenwirkungen der ovariellen Stimulation und Eizellentnahme informiert werden.

\section{b) Besondere Informationspflichten für die Eigenverwendung von Eizellen}

Für den Fall, dass die Spenderin einen Teil der Gameten für den Eigengebrauch konservieren lassen möchte, gelten erweiterte Informationspflichten, die ergänzend zu den vorne genannten hinzutreten (Kapitel V.1 Guide des bonnes pratiques). Die Frau ist demzufolge über nachfolgende Punkte zu informieren:

- über die Bedingungen, unter denen eine solche Konservierung vorgenommen werden kann, und die Regeln über die Verteilung der Gameten. Dafür ist, wie bereits erwähnt, die Anzahl der zur Verfügung stehenden reifen Eizellen von Relevanz. Die Spenderin ist in diesem Kontext insbesondere darüber zu informieren, dass eine Konservierung von Eizellen für die Eigenverwendung unter Umständen nicht möglich ist, was der Fall ist, wenn nicht mehr als fünf reife Eizellen vorhanden sind;

- über die Pflicht, ein Gespräch mit einem Psychiater oder Psychologen des jeweiligen Zentrums zu führen;

- über die Voraussetzungen für die Konservierung und Wiederverwendung der Gameten;

- über die Notwendigkeit, auf die jährlich vom jeweiligen Zentrum versandte Post zu antworten und einen allfälligen Adresswechsel anzuzeigen (hierzu gilt es namentlich Art. R. 1244-7 Abs. 1 CSP zu beachten, der vorsieht, dass die Spenderin jedes Jahr schriftlich kontaktiert werden muss, um abzuklären, ob sie die Konservierung fortsetzen möchte);

- über die gesetzlichen Voraussetzungen zur (Eigen-)Verwendung der konservierten Gameten für den Zugang einer medizinisch unterstützten Fortpflanzung;

- über die Fortsetzung der Konservierung im Hinblick auf eine Spende in den nachfolgenden Fällen:

- Verzicht der Spenderin auf die Konservierung der Eizellen für die spätere Eigenverwendung;

- Überschreitung des Fruchtbarkeitsalters (Art. L. 2141-2 Abs. 2 CSP; siehe dazu Ziff. 4); 
- Ausbleiben einer Antwort auf die jährliche Post des Zentrums (trotz mehrerer Zustellversuche), sofern die Konservierung länger als zehn Jahre andauert;

- Tod der Spenderin.

- Schliesslich ist die Spenderin auch darüber aufzuklären, dass die Konservierung der Gameten für die eigene Vorsorge keine Garantie für eine spätere erfolgreiche medizinisch unterstützte Fortpflanzung darstellt.

\section{c) Einwilligung in die Spende}

Die Spenderin muss nach Erhalt der Informationen eine Einwilligung unterzeichnen. Das dafür zu verwendende Formular hat den nachfolgenden Inhalt zu enthalten (Kapitel V.1 Guide des bonnes pratiques):

- eine Einwilligung in die Spende der Gameten und die Speicherung von persönlichen Daten in einem anonymisierten Spenderdossier; diese Einwilligung umfasst ebenfalls die Zusage, dem Ärzteteam sämtliche der Spenderin nachträglich bekannt gewordenen Krankheiten zu melden, sofern diese über die Gameten auf Dritte übertragen werden können;

- den Wunsch der Spenderin, ob sie über die Resultate der genetischen Untersuchung informiert werden möchte.

Diese Einwilligung ist widerrufbar bis zur Verwendung der Gameten, wobei jeder Widerruf der Einwilligung in die Spende zur Aufhebung der Konservierung der Eizellen führt.

\section{d) Einwilligung in die Konservierung zur Eigenvorsorge}

Möchte die Spenderin einen Teil der Eizellen für die eigene Vorsorge konservieren lassen, so ist, nebst der Einwilligung in die Spende, eine spezifische Einwilligung in die Konservierung zum Eigengebrauch notwendig.

Sie verpflichtet sich darüber hinaus, sämtliche Adressänderungen anzuzeigen und auf die jährlich vom Zentrum versandte Post zu antworten (Art. R. 1244-7 Abs. 1 CSP). Sämtliche Informationen in Bezug auf die Konservierung der Gameten und das Einwilligungsformular sind in einem für die Durchführung von 
Gametenspenden autorisierten Zentrum in einem speziellen Dossier aufzubewahren.

Es ist anzumerken, dass im Falle der Nichtverwendung der für die Eigenvorsorge bestimmten Eizellen diese wahlweise entweder gespendet oder zerstört werden können.

e) Informationspflichten bei einer In-vitro-Fertilisation

Der In-vitro-Fertilisation haben zwingend verschiedene (Informations-)Gespräche mit den Antragstellern voranzugehen. Dabei müssen die Mitglieder des interdisziplinär zusammengesetzten Ärzteteams insbesondere (Art. L. 2141-10 CSP; Kapitel II.1.1 Guide des bonnes pratiques):

- die Motivation der antragstellenden Frau und des antragstellenden Mannes überprüfen und diese auf die Möglichkeit einer Adoption hinweisen;

- das Paar über die Möglichkeit des Erfolgs und Misserfolgs beim Einsatz von Techniken zur medizinisch unterstützten Fortpflanzung aufklären;

- die Antragsteller über Nebenwirkungen und die kurz- und langfristigen Risiken sowie möglichen Beschwerden und damit verbundenen Einschränkungen informieren;

- dem Paar ein Dossier mit folgendem Inhalt abgeben:

- Angaben zu den Gesetzes- und Verordnungsvorschriften zur medizinisch unterstützten Fortpflanzung;

- Beschreibung der Techniken zur medizinisch unterstützten Fortpflanzung;

- Angaben zu den Gesetzes- und Verordnungsvorschriften zur Adoption und Auflistung von Adressen von Einrichtungen, die diesbezüglich weitere Informationen geben können.

Der gestellte Antrag kann durch das Paar erst nach einer Bedenkfrist von einem Monat nach dem letzten Gespräch bestätigt werden. 


\subsubsection{Restriktionen für ausländische Paare}

Das französische Recht sieht keine Restriktionen in Bezug auf die Behandlung ausländischer Paare vor. Angesichts der Verknüpfung mit der Eizellspende dürfte Frankreich allerdings für Frauen aus der Schweiz eine vergleichsweise unattraktive Destination sein.

\subsubsection{Problemfelder der französischen Rechtslage}

Problematisch erscheint zum einen, dass der Grundsatz «kein Social Freezing ohne Eizellspende» dazu führt, dass die Frau mit einer Eizellspende einverstanden sein muss, sofern sie Eizellen für den Eigengebrauch konservieren lassen möchte. Dies könnte für viele Frauen ein wichtiges Argument gegen die Inanspruchnahme des Social Freezing in Frankreich darstellen.

Zum anderen kann eine Konservierung für den Eigengebrauch erst dann erfolgen, wenn mehr als fünf reife Eizellen gespendet werden können. Die Anzahl Eizellen, die für die Eigenvorsorge zur Verfügung stehen, kann dabei sehr gering ausfallen; stehen z.B. acht Eizellen zur Verfügung, so können bloss drei Eizellen davon für die Eigenvorsorge kryokonserviert werden. Berücksichtigt man die Tatsache, dass idealerweise 15 bis 20 Eizellen kryokonserviert werden sollen, um eine realistische Chance auf die Herbeiführung einer Schwangerschaft zu erreichen, so führt die momentane Rechtslage dazu, dass sich eine Frau mehrmaligen Stimulationen unterwerfen muss. Konkret müssen insgesamt 30 bis 40 Eizellen gewonnen werden, um 15 bis 20 Eizellen kryokonservieren zu können.

Zu bedenken ist sodann, dass Voraussetzung zur Inanspruchnahme der künstlichen Fortpflanzung eine rechtsvergleichend ausgesprochen tiefe Altersgrenze ist. Auch aus diesem Grund ist Social Freezing in Frankreich unattraktiv.

In Frankreich zeigt sich damit eine auf die Eizellspende zugeschnittene Rechtslage, wobei die Eizellenvorsorge, wie bereits erwähnt, primär die Eizellspende begünstigen soll. Ob dies unter der momentanen Rechtslage gelingt, wird sich in den nächsten Jahren zeigen. In Anbetracht der strengen Zugangsvoraussetzungen für eine anschliessende medizinisch unterstützte Fortpflanzung und der 
Ausweichmöglichkeiten von Französinnen, die keine Eizellen spenden möchten, zu denken ist vorab an Belgien, sind erhebliche Zweifel angebracht, ob das Ziel des Gesetzgebers auf diese Weise erreicht werden kann.

\subsubsection{Reformbestrebungen}

Im Moment ist eine Reform des «Loi du 7 juillet 2011 relative à la bioéthique» geplant, die voraussichtlich im Jahr 2019 in Kraft treten soll. Einzelheiten zur Reform dürften in der zweiten Jahreshälfte 2018 bekannt werden.

Nachtrag (November 2018): Das französische Comité consultatif national d'éthique hat im Avis 129 vom 18. September 2018 die Empfehlung ausgesprochen, die Verbindung von Eizellspende und Social Freezing zu überprüfen beziehungsweise Social Freezing auch ohne Eizellspende zuzulassen. Als Begründung wird unter anderem angeführt, angesichts der Pflicht zur Spende anlässlich der Eizellenvorsorge könne von einer Kostenlosigkeit der Spende nicht mehr gesprochen werden. Vielmehr handle es sich für die Spenderin gewissermassen um ein «Geschäft». Dennoch bleibt die Stellungnahme des Comité consultatif mit Bezug auf Social Freezing zurückhaltend. Inwiefern die Empfehlungen in der bevorstehenden Revision des französischen Fortpflanzungsmedizingesetzes aufgegriffen werden, war zur Zeit des Abschlusses des vorliegenden Berichts noch offen. 


\subsection{Italien ${ }^{13}$}

\subsubsection{Ausgangslage und Übersicht}

Am 19. Februar 2004 stimmte der Senat des italienischen Parlaments dem Gesetz 40/2004 über die medizinisch unterstützte Fortpflanzung (Legge 19 febbraio 2004, n. 40 - Norme in materia di procreazione medicalmente assistita; nachfolgend: Gesetz 40/2004) zu, dessen Inkrafttreten auf den 10. März 2004 festgesetzt wurde. Damit erfuhr die Fortpflanzungsmedizin in Italien in zweifacher Hinsicht einen Wandel: Einerseits stellte das Gesetz 40/2004 die erste derartige Regelung auf dem Gebiet der assistierten Reproduktion in Italien dar (frühere gesetzgeberische Bestrebungen scheiterten), andererseits verbot man zahlreiche bisher zulässige Methoden, indem man sich für eine restriktive Ausgestaltung des Gesetzes entschied. Nebst dem Gesetz 40/2004 ist unter anderem das vom Gesundheitsministerium im Anschluss erlassene Dekret «Linee guida in materia di procreazione medicalmente assistita» von Bedeutung (vgl. Art. 7 Abs. 1 Gesetz 40/2004; nachfolgend: Linee guida 2015), welches alle drei Jahre aktualisiert werden muss und zuletzt per 1. Juli 2015 überarbeitet wurde (publiziert in Gazzetta Ufficiale Nr. 161 vom 14. Juli 2015).

Die Kryokonservierung von Eizellen wurde in Italien bis 2009 hauptsächlich angewendet, um die Grenzen des Gesetzes 40/2004 zu umgehen, wobei damals in der Regel das Verfahren des slow-freezing angewandt wurde und nicht jenes der Vitrifizierung. Denn das Gesetz 40/2004 statuiert ein Verbot der Kryokonservierung von Embryonen und erlaubt die Erzeugung von maximal drei Embryonen, die zudem gleichzeitig innerhalb eines Zyklus transferiert werden müssen. War der Embryotransfer nicht erfolgreich, musste sich die Frau einer erneuten Stimulation und Eizellentnahme unterziehen. Um die damit verbundenen Belastungen und Risiken zu vermeiden, griff man auf die Kryokonservierung von Eizellen zurück, die gesetzlich nicht auf drei Eizellen beschränkt war. Diese Problematik besteht allerdings seit dem Urteil 151/2009 des Verfas- 
sungsgerichts im Mai 2009 (Sentenza 151/2009 della Corte Costituzionale) nicht mehr, da dieses die Beschränkung auf drei Embryonen und das Erfordernis der gleichzeitigen Implementation aufgehoben hat. Heute wird die Kryokonservierung von Eizellen in Italien auch aus sozialen Gründen praktiziert, verschiedene Fortpflanzungskliniken bewerben das Angebot des Social Freezing.

Gemäss den Linee guida 2015 umfasst der Begriff der medizinisch unterstützten Fortpflanzung «[...] tutti quei procedimenti che comportano il trattamento di ovociti umani, di spermatozoi o embrioni nell'ambito di un progetto finalizzato a realizzare una gravidanza», das heisst alle Verfahren, bei denen menschliche Eizellen, Samenzellen oder Embryonen mit dem Ziel der Herbeiführung einer Schwangerschaft behandelt werden.

Im Anschluss an diese Definition ordnet die Linee guida 2015 die verschiedenen Methoden zur medizinisch unterstützten Fortpflanzung - je nach Komplexität und Intensität des Eingriffs - in drei verschiedene Ebenen ein: Sowohl die Kryokonservierung weiblicher Gameten als auch die In-vitro-Fertilisation werden der zweiten Ebene (Methoden, die eine lokale Betäubung und/oder Allgemeinanästhesie erfordern) zugeteilt. Damit würde das gesamte Verfahren des Social Freezing unter den Begriff der medizinisch unterstützten Fortpflanzung fallen und Gegenstand des Gesetzes 40/2004 bilden. Allerdings gilt es, nebst der Auflistung der einzelnen Techniken auch die vorangehende Definition zu beachten, welche verlangt, dass mit der Inanspruchnahme der erwähnten Techniken das Ziel einer Schwangerschaft verfolgt werden muss. Im Falle des Social Freezing ist dies in den ersten beiden Phasen nicht der Fall, geht es doch um eine blosse Vorsorge und nicht um die konkrete Herbeiführung einer Schwangerschaft (vgl. auch die Argumentation zu Art. 2 lit. a sFMedG, wo eine ähnliche Definition der medizinisch unterstützten Fortpflanzung vorgesehen ist). Deshalb ist wohl dafürzuhalten, wenngleich die italienische Rechtswissenschaft sich mit dieser Frage, soweit ersichtlich, noch nicht auseinandergesetzt hat, dass die ersten beiden Phasen des Social Freezing vom Anwendungsbereich des Gesetzes 40/2004 ausgenommen werden und das Gesetz 40/2004 einzig in Phase 3 zur Anwendung kommt. Dies scheint auch die Auffassung in der Praxis zu sein, wird doch, wie erwähnt, Social Freezing in Italien praktiziert und aktiv beworben. 


\subsubsection{Zulässigkeit einer rein sozialen Indikation}

Die Entnahme und Konservierung der Eizellen (Phase 1 und 2) fällt, wie soeben erörtert, nach allgemein vertretener Auffassung nicht unter das Gesetz 40/2004. Dies hat insbesondere zur Folge, dass die in Art. 4 Abs. 1 (objektive Voraussetzungen) und Art. 5 (subjektive Voraussetzungen) Gesetz 40/2004 vorgesehenen Zugangsschranken nicht zur Anwendung kommen und damit zum Zeitpunkt der Entnahme und Konservierung keine Infertilität vorliegen muss. Mangels eines Verbotes beziehungsweise einer spezifischen Regelung der blossen Entnahme und Konservierung der Eizellen ist dieser erste Schritt des Social Freezing in Italien zulässig.

Die Kryokonservierung findet ausschliesslich in Fortpflanzungsmedizinzentren statt. Art. 10 und 11 Gesetz 40/2004 schreiben eine Registrierungspflicht für private und öffentliche Einrichtungen vor, die medizinisch assistierte Reproduktion anbieten (siehe dazu die Website des «Registro Nazionale Procreazione Medicalmente Assistita»).

Für das konkrete Vorgehen gelten dieselben Regeln wie für alle fortpflanzungsmedizinischen Verfahren, das heisst insbesondere: Es finden ein Aufklärungsgespräch, diagnostische Untersuchungen und eine HormonprofilBewertung statt, und es wird ein Hormonstimulationsprotokoll geführt.

\subsubsection{Aufbewahrungsfrist}

Das italienische Recht sieht keine Maximaldauer für die Aufbewahrung von Eizellen vor. Allerdings ist die anschliessende In-vitro-Fertilisation an gewisse Altersgrenzen gebunden.

\subsubsection{Voraussetzungen für die In-vitro-Fertilisation}

Art. 4 Abs. 1 Gesetz 40/2004 umschreibt die objektiven und Art. 5 Gesetz 40/2004 die subjektiven Anforderungen für den Zugang zur medizinisch unterstützten Fortpflanzung. 
Gemäss Art. 4 Abs. 1 Gesetz 40/2004 sind medizinisch unterstützte Fortpflanzungsmedizintechniken nur erlaubt, wenn eine medizinisch bestätigte Unfruchtbarkeit vorliegt, die nicht anders beseitigt werden kann. Dass in Zusammenhang mit Social Freezing die Unfruchtbarkeit auf dem nunmehr fortgeschrittenen Alter beruht, steht der Anwendung der In-vitro-Fertilisation nicht unbedingt entgegen. Die Frau muss jedoch bei der In-vitro-Fertilisation noch im grundsätzlich fruchtbaren Alter sein («in età potenzialmente fertile»), wobei das Gesetz keine konkrete Altersgrenze festlegt. Die Gesetzesbestimmung rechtfertigt sich dadurch, dass das Kind nicht zu alte Eltern haben soll, damit diese noch in der Lage sind, sich um seine Bedürfnisse zu kümmern. Zudem sollen die Risiken, die eine Schwangerschaft in fortgeschrittenem Alter mit sich bringt, reduziert werden. Da das Gesetz selber keine konkrete Altersgrenze enthält, ist diese nicht vollständig geklärt beziehungsweise die Festlegung erfolgt durch die Regionen und autonomen Provinzen. Dadurch ergaben sich zunächst sehr unterschiedliche Alterslimiten. Derartige Unterschiede in Bezug auf die Altersgrenze sowie die nach regionaler Gesetzgebung teilweise damit verbundene Unterscheidung zwischen homologer und heterologer Insemination verstossen jedoch, wie das Verwaltungsgericht in Venedig entschieden hat (T.A.R. Venezia 8.5.2015, n. 501, FA 2015, 5, 1515; FI 2015, 7-8, III, 423), gegen den Gleichbehandlungsgrundsatz. Nach einem von der Konferenz der Regionen und autonomen Provinzen ausgearbeiteten Dokument wird die Grenze für die In-vitroBefruchtung auf 50 Jahre festgelegt.

\subsubsection{Spezifische Beratungspflichten}

Der informierte Konsens bei fortpflanzungsmedizinischen Verfahren wurde in Italien neu geregelt, siehe: DECRETO 28 dicembre 2016, n. 265, Regolamento recante norme in materia di manifestazione della volontà di accedere alle tecniche di procreazione medicalmente assistita, in attuazione dell'articolo 6, comma 3, della legge 19 febbraio 2004, n. 40. (17G00024) (GU Serie Generale n. 40 del 17-02-2017) (entrata in vigore del provvedimento: 04/03/2017).

Art. 6 Gesetz 40/2004 definiert die Grundsätze zum informierten Konsens, zusammen mit dem DECRETO 28 dicembre 2016, n. 265 Regolamento recante norme in materia di manifestazione della volontà di accedere alle tecniche di procreazione medicalmente assistita, in attuazione dell'articolo 6, comma 3 , 
della legge 19 febbraio 2004, n. 40. Art. 14 Abs. 8 Gesetz 40/2004 sieht vor, dass die Kryokonservierung von Eizellen einer vorherigen schriftlichen Einwilligung bedarf, die sich nach Art. 6 Gesetz 40/2004 zu richten hat.

\subsubsection{Restriktionen für ausländische Paare}

Das italienische Recht sieht keine Restriktionen in Bezug auf ausländische Paare vor. Wie oft Social Freezing von Schweizer Frauen in Italien in Anspruch genommen wird, ist nicht bekannt.

\subsubsection{Reformbestrebungen}

Gemäss Art. 7 Abs. 3 Gesetz 40/2004 muss die Linee guida mindestens alle drei Jahre aktualisiert werden. Im Übrigen sind keine Reformbestrebungen bekannt.

\subsection{Deutschland}

Im deutschen Recht fehlt bislang eine umfassende fortpflanzungsmedizinische Gesetzgebung. Stattdessen wird die Reproduktionsmedizin heute unter anderem im Verfassungs-, Embryonenschutz-, Gewebe-, Arzneimittel- und Familienrecht sowie im ärztlichen Berufsrecht unvollständig und häufig auch nicht zweckmässig geregelt. Rechtliche Rahmenbedingungen betreffend die Entnahme und Konservierung von Eizellen bestehen weitgehend nicht, ist doch das Embryonenschutzgesetz auf unbefruchtete Eizellen nicht anwendbar. Gesetzliche Vorgaben zur hormonellen Stimulation und Eizellentnahme fehlen vollständig. Erschwerend kommt hinzu, dass sich die rechtswissenschaftliche Literatur und Rechtsprechung in Deutschland, soweit ersichtlich, bisher kaum umfassend mit Social Freezing befasst hat. Es ist deshalb mehr als fraglich, ob sich aus dem deutschen Medizin- und Fortpflanzungsrecht neue Erkenntnisse für eine allfällige Gesetzesrevision in der Schweiz gewinnen lassen. 
Immerhin ist davon auszugehen, dass für die Eizellenvorsorge die normalen Regeln des Arztrechts, insbesondere die Regeln des Patientenrechtegesetzes, uneingeschränkt Anwendung finden. Damit sind auch Aufklärungs-, Einwilligungs- und Dokumentationspflichten des Arztes verbunden.

Lediglich die Ärzteschaft ist im Bereich der Fortpflanzungsmedizin konkret regulierend tätig geworden, indem der Vorstand der Bundesärztekammer die am 1. Juni 2018 veröffentlichte «Richtlinie zur Entnahme und Übertragung von menschlichen Keimzellen im Rahmen der assistierten Reproduktion» gemäss § 16b TPG (Transplantationsgesetz) beschlossen hat. Die veraltete «(Muster-) Richtlinie zur Durchführung der assistierten Reproduktion» aus dem Jahr 2006 wurde damit gegenstandslos. Bei der neuen Richtlinie handelt es sich wiederum lediglich um Standesrecht, sodass auch in absehbarer Zukunft eine demokratisch legitimierte und verbindliche Gesetzgebung nach wie vor fehlen wird. Zudem ist zu beachten, dass die Richtlinie einzig den «allgemein anerkannten Stand der Erkenntnisse der medizinischen Wissenschaft [...] zur Entnahme von Geweben und deren Übertragung» feststellt (§ 16b TPG). Gesellschaftspolitische Fragen, die bislang keine klare rechtliche Regelung erfahren haben, werden in der Richtlinie bewusst nicht beantwortet, da dies in die Kompetenz des Gesetzgebers fällt. Die rechtlichen Unklarheiten in Bezug auf Social Freezing dürften damit grösstenteils fortbestehen.

Insgesamt zeigt sich in Deutschland ein komplexer und uneinheitlicher Regelungsansatz der Reproduktionsmedizin, was insbesondere bei der Entstehung neuer Methoden der medizinisch unterstützten Fortpflanzung, wie z.B. des Social Freezing, zu Unsicherheiten bei der Rechtsanwendung und Interpretation der Erlasse führt. Allgemein wird aber davon ausgegangen, dass mangels eines ausdrücklichen Verbots sowohl die Eizellentnahme wie auch die Aufbewahrung und spätere Verwendung von Eizellen erlaubt sind. Dementsprechend wird Social Freezing von verschiedenen Kinderwunschzentren angeboten. Die Nachfrage dürfte allerdings eher gering ausfallen. 


\subsection{Belgien}

\subsubsection{Ausgangslage und Überblick}

Die Fortpflanzungsmedizin wird in Belgien massgeblich durch das «Loi relative à la procréation médicalement assistée et à la destination des embryons surnuméraires et des gamètes» vom 6. Juli 2007 (nachfolgend: Loi-PMA) geregelt. Dabei verfolgte der Gesetzgeber einen liberalen Ansatz, der sich insbesondere durch Flexibilität, Transparenz und Wahrung der persönlichen Autonomie auszeichnet. Diese Ausgestaltung ist im Wesentlichen auf zwei Ursachen zurückzuführen: Einerseits hat der späte Erlass des Gesetzes dazu geführt, dass die Medizin von den bisher existierenden Freiheiten Gebrauch gemacht hatte, weshalb es teilweise nur schwer möglich war, nachträglich Einschränkungen vorzunehmen. Andererseits hatte die im belgischen Familienrecht generell vorherrschende Liberalität einen Einfluss.

Dementsprechend ist Social Freezing in Belgien weitgehend zulässig. Einzig die grundsätzliche Aufbewahrungsfrist von zehn Jahren, die Altersgrenzen, insbesondere die Grenze von knapp 48 Jahren für den Embryotransfer, und die Freiheit der Zentren, restriktivere als die im Gesetz vorgesehenen Bedingungen zu statuieren, können als Hindernisse betrachtet werden.

\subsubsection{Zulässigkeit einer rein sozialen Indikation}

\section{a) Gesetzliche Regelung}

Die Eizellentnahme und Kryokonservierung aus sozialen und damit nicht medizinisch indizierten Gründen ist nach belgischem Recht erlaubt (Art. 37 und Art. 40 Abs. 1 Loi-PMA). So legt das Gesetz ausdrücklich fest, dass Gameten unter anderem im Hinblick auf eine Kryokonservierung und einen späteren Kinderwunsch entnommen werden können. Dabei dürfen die Gameten einzig gemäss der zwischen den Wunscheltern und dem beigezogenen Fertilitätszentrum abgeschlossenen Vereinbarung verwendet werden (Art. 38 Loi-PMA). 
Auch die allgemeinen Zugangsschranken zur medizinisch assistierten Fortpflanzung stehen einer Entnahme und Kryokonservierung nicht entgegen. So sieht der belgische Gesetzgeber, abgesehen von verschiedenen Altersgrenzen, keinerlei Beschränkungen vor und vertraut stattdessen auf die Einschätzung des Ärzteteams und des jeweiligen Fertilitätszentrums (siehe dazu sogleich, b):

- Die medizinisch assistierte Reproduktion ist von Gesetzes wegen nicht auf verheiratete beziehungsweise unverheiratete Paare beschränkt und kann namentlich auch von alleinstehenden Frauen in Anspruch genommen werden.

- Die Entnahme der Eizellen kann frühestens bei Volljährigkeit (Vollendung des 18. Lebensjahres) und spätestens an jenem Tag, der dem 46. Geburtstag vorangeht, erfolgen (Art. 4 Abs. 1 Loi-PMA).

- Vom Volljährigkeitserfordernis kann ausnahmsweise abgesehen werden, wenn die Entnahme im Hinblick auf eine Kryokonservierung von Gameten aus medizinischen Gründen erfolgt (Art. 4 Abs. 4 Loi-PMA).

Die Altersgrenzen wurden in den Vorbereitungsarbeiten zum Gesetz vor allem mit ethischen und medizinischen Argumenten, z.B. abnehmende Qualität der Eizellen im Alter, gerechtfertigt.

\section{b) Einschränkende Regelungen einzelner Fertilitätszentren}

Die Fertilitätszentren können die Zugangskriterien für eine Behandlung innerhalb der weiten Grenzen des Gesetzes grösstenteils selbst bestimmen, indem sie sich in Bezug auf die an sie gerichteten Anträge auf die Gewissensklausel berufen (Art. 5 Abs. 1 Teilsatz 2 Loi-PMA). Es steht damit den Fertilitätszentren letztlich frei, ob sie z.B. alleinstehende Frauen behandeln möchten, wobei diesbezüglich jedes Zentrum einen eigenen Ansatz verfolgt.

Als Ausgleich zur Möglichkeit, sich auf die Gewissensklausel berufen zu können, sind die Fertilitätszentren zur Transparenz verpflichtet (Art. 5 Abs. 1 Teilsatz 1 Loi-PMA) und müssen die Antragstellerin beziehungsweise die Antragsteller binnen Monatsfrist, nach der Entscheidung des beigezogenen Arztes, über eine Weigerung informieren (Art. 5 Abs. 2 Loi-PMA). Diese muss schriftlich erfolgen und hat zwingend die nachfolgenden Angaben zu enthalten (Art. 5 Abs. 3 Loi-PMA): 
- die medizinischen Gründe, weshalb die Weigerung erfolgte;

- die in Art. 5 Abs. 1 Loi-PMA erwähnte Berufung auf die Gewissensklausel;

- sofern von der Antragstellerin beziehungsweise den Antragstellern gewünscht, die Adresse eines anderen Fertilitätszentrums, an das sie sich wenden können.

\subsubsection{Aufbewahrungsfrist}

Die Frist für die Aufbewahrung der entnommenen Eizellen beträgt zehn Jahre und beginnt mit dem Tag der Kryokonservierung (Art. 46 Abs. 1 Loi-PMA). Nach Ablauf der Frist führt das Zentrum die in der Vereinbarung festgelegte Anweisung aus: Die Gameten können zum einen vernichtet oder zum anderen für die wissenschaftliche Forschung oder eine Gametenspende bereitgestellt werden (Art. 40 Abs. 2 Loi-PMA).

Es besteht die Möglichkeit, dass die Frist gemäss Art. 46 Loi-PMA aufgrund besonderer Umstände verlängert wird. Erforderlich ist hierfür ein schriftlicher Antrag an das Fertilitätszentrum, das anschliessend über eine Verlängerung entscheidet (Art. 47 Loi-PMA). Es liegt im Ermessen des Fertilitätszentrums, ob «besondere Umstände» für eine Verlängerung vorliegen. Daher ist davon auszugehen, dass es in der Praxis relativ einfach ist, eine Verlängerung der Aufbewahrung aus sozialen Gründen und mit Blick auf das nunmehr fortgeschrittene Alter der betroffenen Frau zu verlangen.

\subsubsection{Voraussetzungen für die In-vitro-Fertilisation}

Es kann diesbezüglich weitgehend auf die Ausführungen zu Ziff. 2 verwiesen werden, wonach das belgische Recht, abgesehen von verschiedenen Altersgrenzen, keinerlei Beschränkungen vorsieht und stattdessen auf die Einschätzung des Ärzteteams und des jeweiligen Fertilitätszentrums vertraut. Das Gesetz sieht ergänzend die folgenden beiden Altersgrenzen vor: 
- Die Anträge auf Embryotransfer beziehungsweise Insemination müssen spätestens an jenem Tag eingereicht werden, der dem 46. Geburtstag der Antragstellerin vorangeht (Art. 4 Abs. 2 Loi-PMA).

- Der anschliessende Embryotransfer beziehungsweise die anschliessende Insemination hat vor jenem Tag zu erfolgen, der dem 48. Geburtstag der Antragstellerin vorangeht (Art. 4 Abs. 3 Loi-PMA).

\subsubsection{Spezifische Beratungspflichten}

In verschiedenen Bestimmungen des Loi-PMA sind Informationspflichten geregelt, die dann zum Zuge kommen, wenn das Fertilitätszentrum beschliesst, dem Antrag auf medizinisch assistierte Fortpflanzung Folge zu leisten (vgl. Art. 6 Abs. 2 Ziff. 1 Loi-PMA). Abgesehen davon, dass dem Paar beziehungsweise dem Wunschelternteil bei medizinisch assistierter Fortpflanzung immer eine psychologische Begleitung anzubieten ist (Art. 6 Abs. 2 Ziff. 2 Loi-PMA), bestehen keine spezifischen Informations- oder Aufklärungspflichten in Zusammenhang mit Social Freezing. Sicherzustellen ist indessen bei jedem medizinischen Schritt, dass dieser von der Zustimmung der Wunschelternteile abgedeckt ist.

Zudem müssen die Wunscheltern beziehungsweise der Wunschelternteil und das beigezogene Fertilitätszentrum vor jedem medizinischen Behandlungsschritt eine Vereinbarung abschliessen (Art. 7 Abs. 1 Loi-PMA), in der unter anderem die Personalien, das Alter und die Adresse der Antragstellerin sowie die Kontaktinformationen des Reproduktionszentrums zu vermerken sind (Art. 7 Abs. 2 Loi-PMA). Ebenso muss die gewünschte Methode zur medizinisch assistierten Reproduktion angegeben werden.

\subsubsection{Restriktionen für ausländische Paare}

Das belgische Recht sieht keine Restriktionen in Bezug auf ausländische Paare vor. Belgien ist damit eine attraktive Destination, insbesondere wohl für Frauen aus Frankreich, wo die Rechtslage ausgesprochen restriktiv ist. 


\subsubsection{Reformbestrebungen}

Soweit ersichtlich, gibt es aktuell keine Reformbestrebungen in Bezug auf Social Freezing.

\subsection{Spanien ${ }^{14}$}

Spanien ist seit vielen Jahren bekannt für das auch gezielt an Ausländerinnen gerichtete Angebot verschiedener fortpflanzungsmedizinischer Verfahren. Die Rechtslage in Spanien ist ausgesprochen liberal; zahlreiche Zentren bieten entsprechende Behandlungen an und bewerben diese offensiv und oftmals auch in deutscher Sprache.

\subsubsection{Zulässigkeit einer rein sozialen Indikation}

Die Entnahme und Konservierung von Eizellen zur reinen «Vorsorge» ist in Spanien erlaubt und weit verbreitet. Die entsprechenden rechtlichen Rahmenbedingungen finden sich in Kapitel III («Kryokonservierung und weitere Techniken zur medizinisch unterstützten Fortpflanzung»; Art. 11-13) des Gesetzes 14/2006 über die Techniken zur assistierten Reproduktion vom 26. Mai 2006 (nachfolgend: Gesetz 14/2006). Die Gesetzesbestimmungen nehmen zwar nicht ausdrücklich auf die Eizellenvorsorge Bezug, regulieren aber die Lagerbedingungen sowie die Verwendung von kryokonservierten Eizellen und Ovarialgewebe (Art. 11 Ziff. 3-7 Gesetz 14/2006). Dies impliziert, dass die Kryokonservierung von Eizellen aus rein präventiven Gründen zulässig ist und den allgemeinen Vorschriften über die Techniken zur assistierten Reproduktion unterliegt. 


\subsubsection{Aufbewahrungsfrist}

Das spanische Recht sieht anstelle einer starren Altersgrenze eine medizinische Beurteilung im Einzelfall vor. Damit können Eizellen und Ovarialgewebe so lange gelagert werden, bis der für die Behandlung zuständige Arzt in Übereinstimmung mit den beigezogenen unabhängigen Spezialisten, die nicht dem mit der Behandlung betrauten Zentrum angehören dürfen, feststellt, dass die Patientin die medizinischen Voraussetzungen für eine künstliche Fortpflanzung nicht mehr erfüllt (Art. 11 Ziff. 3 Gesetz 14/2006).

In Spanien empfehlen die Verbände zur assistierten Fortpflanzung einschliesslich der Sociedad Española de Fertilidad (SEF) und der Asociación Nacional de Clínicas de reproducción asistida (ANACER), Frauen spätestens bis zum 50. Altersjahr zu behandeln, wobei aber jeder Fall einer eigenen Wertung und Einschätzung bedarf. Letzteres entspricht auch dem momentanen Trend in der Praxis spanischer Privatkliniken, die in Bezug auf die maximale Lagerdauer vor allem darauf abstellen, wie effektiv beziehungsweise Erfolg versprechend eine allfällige Behandlung wäre.

Die von spanischen Kliniken zur Verfügung gestellten Informationen zu Social Freezing und die zwischen den Kliniken und den Patientinnen geschlossenen Verträge sehen dementsprechend meist keine konkrete Aufbewahrungsfrist vor. Häufig wird einzig der Wortlaut von Art. 11 Ziff. 3 Gesetz 14/2006 übernommen und auf die Risiken hingewiesen, die sich aus der Inanspruchnahme von medizinisch unterstützter Fortpflanzung in «fortgeschrittenem Alter» ergeben.

Schliesslich ist die Aufbewahrung der Eizellen auch von den Wünschen der Frau abhängig. So kann eine Frau, die sich gegen eine In-vitro-Fertilisation entscheidet, wählen, ob sie die Eizellen für Fortpflanzungs- oder Forschungszwecke spenden oder die Lagerung ohne weitere Verwendung beenden möchte (Art. 11 Ziff. 4 lit. b, c und d Gesetz 14/2006). Hat es die Frau versäumt, eine Entscheidung zu treffen, und ist das Zeitfenster für eine Behandlung nach Art. 11 Ziff. 3 Gesetz 14/2006 überschritten worden, so sieht das spanische Recht vor, dass die Lagerung der Eizelle ohne weitere Verwendung beendet wird. 


\subsubsection{Voraussetzungen für die In-vitro-Fertilisation}

Es kommen bei der In-vitro-Fertilisation die allgemeinen gesetzlichen Vorschriften zur medizinisch unterstützten Reproduktion zur Anwendung: Das spanische Recht sieht keine Beschränkung auf Patientinnen vor, die an Unfruchtbarkeit leiden oder aus anderen medizinischen Gründen nicht auf natürlichem Weg schwanger werden können. Vielmehr kann jede mindestens 18-jährige und voll urteilsfähige Frau ungeachtet ihres Zivilstandes und ihrer sexuellen Orientierung (Art. 6 Ziff. 1 Gesetz 14/2006) eine medizinisch unterstützte Fortpflanzung in Anspruch nehmen, sofern sie freiwillig, bewusst und ausdrücklich ihre Einwilligung dazu erteilt hat.

Eine Inanspruchnahme kommt aber nur infrage, sofern (i) eine hinreichende Erfolgswahrscheinlichkeit besteht, (ii) die Behandlung kein ernstes Risiko für die physische oder psychische Gesundheit der Frau oder ihre Nachkommen birgt und (iii) die Frau vollumfänglich über die Erfolgswahrscheinlichkeit und die Risiken sowie die langfristigen Auswirkungen der Behandlung informiert wurde (Art. 3 Ziff. 1 Gesetz 14/2006). Im Falle einer In-vitro-Fertilisation und damit verwandter Techniken darf pro Zyklus ein Maximum von drei Präembryonen transferiert werden (Art. 3 Ziff. 2 Gesetz 14/2006).

\subsubsection{Spezifische Beratungspflichten}

Das spanische Recht verpflichtet die Kliniken dazu, den Patientinnen vor Behandlungsbeginn obligatorische Informationen und Beratungen bereitzustellen. Die Informationen müssen biologische, juristische und ethische Aspekte umfassen sowie die finanziellen Kosten der Behandlung präzisieren (Art. 3 Ziff. 3 Gesetz 14/2006). Die Informationsbroschüren und die Einverständniserklärung müssen angemessen aufbereitet, einfach zugänglich sowie für jedermann, insbesondere auch für Personen ohne juristisches Fachwissen leicht verständlich sein (Art. 11 Ziff. 7 Gesetz 14/2006).

Die von den spanischen Kliniken typischerweise verwendeten Einverständniserklärungen folgen den von Verbänden zur assistierten Fortpflanzung entworfenen Standarderklärungen, hauptsächlich diejenigen der Sociedad Española de Fertilidad, und beinhalten die folgenden Angaben: Beschreibung des Verfah- 
rens; allfällig erforderliche medizinische Indikationen; Erfolgschancen einschliesslich der Schwangerschaftsraten und der Überlebensquoten von Eizellen nach dem Auftauen; allgemeine Risiken; spezifische Risiken im jeweiligen Fall; Behandlungskosten in Bezugnahme auf ein im Vorfeld präzisiertes Budget; juristische Informationen sowie Alternativen im Falle einer erfolglosen Behandlung.

\subsubsection{Restriktionen für ausländische Paare}

In Spanien existieren keine Restriktionen. Im Gegenteil, Spanien ist eine der Topdestinationen für «Fortpflanzungstourismus» in Europa, und viele spanische Kliniken bieten eine umfassende Betreuung in verschiedenen Sprachen an.

8.8.6 Reformbestrebungen

Im Moment gibt es in Spanien keine Reformbestrebungen.

\subsection{Tschechische Republik ${ }^{15}$}

\subsection{1}

Ausgangslage und Überblick

Medizinisch unterstützte Fortpflanzung wird in der Tschechischen Republik bereits seit langer Zeit praktiziert. Die Auffassung in der Gesellschaft ist entsprechend liberal und es wird ausgesprochen viel Wert auf die Selbstbestim-

15 Die Ausführungen zum tschechischen Recht wurden mit kleineren redaktionellen Anpassungen aus dem Gutachten von JUDr. Lenka Westphalová, Ph.D., Juristische Fakultät Olomouc (Olmütz), Tschechien, übernommen. 
mung der Frau in Reproduktionsangelegenheiten gelegt. Grund für die vorherrschende Liberalität sind unter anderem die politische und kulturelle Geschichte der Republik sowie der schon immer hohe medizinische Standard in der Reproduktionsmedizin.

\subsubsection{Zulässigkeit einer rein sozialen Indikation}

Der Zugang zur medizinisch unterstützten Fortpflanzung richtet sich nach dem Gesetz Nr. 373/2011 über die spezifischen Gesundheitsdienste vom 6. November 2011 (in Kraft seit 1. April 2012; nachfolgend: Gesetz Nr. 373/2011). Das tschechische Recht unterscheidet dabei nicht danach, ob die Entnahme und Konservierung aus medizinischen Gründen oder aus rein «sozialen» Motiven erfolgt (vgl. § 3 Abs. 4 lit. c Gesetz Nr. 373/2011). Daraus ergibt sich ohne Weiteres die Zulässigkeit der Eizellenvorsorge.

Ungeklärt ist bislang lediglich die Frage, wie der zwischen der Frau und dem Anbieter abgeschlossene Vertrag zivilrechtlich zu qualifizieren ist - infrage kommt insbesondere ein Gesundheitsvertrag (vgl. § 2636 CZ/BGB).

\subsubsection{Aufbewahrungsfrist}

Das Gesetz sieht keine Maximaldauer für die Aufbewahrung von Eizellen vor. Die Aufbewahrungsfrist der Eizellen wird deshalb vertraglich festgesetzt, wobei im Regelfall eine Dauer von drei Jahren vereinbart wird. Die Patientin wird auf den Ablauf der Frist hingewiesen, sodass sie gegebenenfalls die Aufbewahrung verlängern kann. Tut sie dies nicht, so werden die Eizellen vernichtet.

\subsubsection{Voraussetzungen für die In-vitro-Fertilisation}

Nach tschechischem Recht kann eine Frau eine In-vitro-Fertilisation nur im fruchtbaren Alter, das heisst bis zur Erreichung des 49. Lebensjahres, in Anspruch nehmen. Es muss dafür ein schriftlicher Antrag eines verheirateten oder 
unverheirateten Paares vorliegen, wobei jeder Befruchtungsversuch eines neuen Antrags bedarf.

Vor der In-vitro-Fertilisation treffen den Anbieter verschiedene Informationspflichten in Bezug auf die anzuwendende Methode, die möglichen Risiken der In-vitro-Fertilisation, die Möglichkeiten, wie mit überzähligen Embryonen verfahren werden kann, und die Kosten. Das Paar muss auch über die gesetzliche Regelung zur Feststellung der Vaterschaft informiert werden, wonach der Mann, der die Zustimmung zur In-vitro-Fertilisation erteilt hat, auch Vater des Kindes ist ( $\$ 778 \mathrm{CZ/BGB).} \mathrm{Nach} \mathrm{Erhalt} \mathrm{dieser} \mathrm{Informationen} \mathrm{muss} \mathrm{das} \mathrm{Paar}$ ein entsprechendes Protokoll unterschreiben und kann daraufhin der In-vitroFertilisation zustimmen. Die Befruchtung kann entweder mit eigenen Keimzellen erfolgen oder es kann auf einen anonymen Spender zurückgegriffen werden.

\subsubsection{Spezifische Beratungspflichten}

Das Gesetz sieht im Hinblick auf Social Freezing keine spezifischen Informationspflichten vor. Allerdings erfolgt in der Praxis auch hier eine Beratung, wo die Patientin über ihren Gesundheitszustand sowie die Erfolgschancen des Social Freezing und insbesondere darüber, dass eine erfolgreiche In-vitroFertilisation nicht garantiert werden kann und die Eizellen die Kryokonservierung unter Umständen nicht überleben, informiert wird. Zudem wird ihr das Verfahren erläutert und sie wird über die möglichen Risiken und Nebenwirkungen aufgeklärt. Schliesslich wird die Interessentin über die Preise informiert und es wird darauf hingewiesen, dass die gesetzliche Krankenversicherung die entstehenden Kosten nicht übernimmt. Für die Informationspflichten in Bezug auf eine anschliessende In-vitro-Fertilisation kann auf Ziff. 2 verwiesen werden.

\subsubsection{Restriktionen für ausländische Paare}

Es gibt keinerlei Einschränkungen in Bezug auf ausländische Paare (Vertragsfreiheit). Viele tschechische Anbieter werben im Internet unter anderem auch in deutscher Sprache. Die Kosten für Social Freezing sind vergleichsweise sehr tief. Sie belaufen sich ungefähr auf 1650 Euro für ein Erstgespräch, ein Stimula- 
tionsprotokoll (exkl. Stimulationsmedikamente), Serologie im Zeitraum der Eizellentnahme, Eizellentnahme, Anästhesie, Belegbett, Vitrifizierung von bis zu zwei Röhrchen mit Eizellen (Straw) inklusive Lagerung bis maximal fünf Jahre (vgl. https://www.pronatal.cz/de/preisliste, abgerufen am 6. Mai 2018). Jedes weitere Jahr der Aufbewahrung kostet 50 Euro. Die tiefen Kosten und die liberale gesetzliche Regelung machen Tschechien zu einem attraktiven Land für Fortpflanzungsmedizintourismus.

\subsubsection{Reformbestrebungen}

Es gibt aktuell keine Reformbestrebungen in der Tschechischen Republik. 


\section{Schlussfolgerungen}

Nachfolgend werden die Ergebnisse der Studie entlang der Forschungsfragen zur Technologie, zur Nachfrage sowie zu den ethischen, gesellschaftlichen, finanziellen und rechtlichen Implikationen des Social Freezing präsentiert.

\subsection{Beantwortung der Forschungsfragen}

In diesem Kapitel werden die Forschungsfragen von TA-SWISS beantwortet. In die Antworten fliessen die in Kapitel 3 bis 9 beschriebenen Befunde ein.

\subsubsection{Chancen und Risiken der Technologie}

Unter Social Freezing wird das vorsorgliche Einfrieren eigener Eizellen bezeichnet, ohne dass dafür eine medizinische Notwendigkeit vorliegt. Die Eizellen können zu einem späteren Zeitpunkt aufgetaut und in vitro befruchtet werden. Dadurch können Frauen in einem Alter schwanger werden, in dem sie normalerweise bereits unfruchtbar wären. Anstelle des Social Freezing wird aus diesem Grund teilweise von einer Fertilitätsreserve aus sozialer Indikation gesprochen. Unter sozialer Indikation werden berufliche Gründe oder der fehlende Partner für die Familiengründung subsumiert, die gesunde Frauen dazu bewegen, ihre Eizellen einfrieren zu lassen. Demgegenüber lassen Krebspatientinnen ihre Eizellen auf eine medizinische Indikation gestützt einfrieren.

Der medizinische Prozess des Social Freezing besteht aus drei zeitlich abgegrenzten Phasen. In der ersten Phase erfolgt die Hormonbehandlung zur Stimulation der Eierstöcke (ovarielle Stimulation). Danach werden diese über die Scheide mit einer Nadel punktiert und die Eizellen abgesaugt. Die zweite Phase umfasst die Kryokonservierung der Eizellen. In der dritten Phase werden die Eizellen aufgetaut und mit den Spermien des Wunschvaters in der Glasschale in vitro befruchtet. Reproduktionsmedizinische Verfahren durchlaufen seit 40 Jahren die erste und dritte Phase. Die Kryokonservierung der Eizellen wird hin- 
gegen erst seit gut zehn Jahren praktiziert und hat eine breite Anwendung des Social Freezing erst ermöglicht. Ausschlaggebend für diese Entwicklung war ein neues Verfahren zur Kryokonservierung der Eizellen. Mit der Vitrifikation werden die Eizellen schockgefroren, wodurch die Bildung von zerstörerischen Eiskristallen in der Eizelle vermieden wird. Dadurch unterscheiden sich die Schwangerschaftsraten nach der Befruchtung der kryokonservierten Eizellen in vitro kaum noch von denjenigen mit frischen Eizellen.

Nachfolgend werden die Risiken und Chancen, gestützt auf wissenschaftliche Artikel, beschrieben. Ergänzend dazu finden sich Informationen aus einer Onlinebefragung bei Reproduktionsmedizinerinnen und -medizinern sowie explorativen Interviews mit Reproduktionsmedizinern und Frauen, die die Technologie bereits genutzt haben.

Welche Risiken bestehen für Frauen bei der ovariellen Stimulation und der Eizellentnahme?

Die Risiken in Zusammenhang mit der Hormonstimulation und der Eizellentnahme sind gering. Vier Risiken werden in der Literatur diskutiert. Ein schweres Überstimulationssyndrom tritt in weniger als 1 Prozent der Fälle auf. Ebenso liegt das Risiko für Komplikationen bei der Eizellentnahme bei unter 1 Prozent. Ein erhöhtes Krebsrisiko in der Brust, den Eierstöcken oder der Gebärmutterschleimhaut sowie eine Einschränkung der Fertilität wurden bisher nicht festgestellt.

Welche Risiken bestehen für das Kind nach der Befruchtung der Eizellen in vitro?

Ein wichtiges, mit der Technologie verbundenes Risiko ist der im Vergleich zur natürlichen Zeugung erhöhte Anteil von Mehrlingsschwangerschaften nach einer Befruchtung der Eizellen in vitro. Mehrlingsschwangerschaften erhöhen die Prävalenz von Schwangerschaftsvergiftungen, Frühgeburtlichkeit und niedrigem Geburtsgewicht. Diese Komplikationen führen zu mehr Herz-Kreislauf-Erkrankungen und Todesfällen im späteren Leben. Es besteht allerdings international eine Tendenz, nur noch einen Embryo in die Gebärmutter zu übertragen, womit dieses Risiko künftig gesenkt werden dürfte. Allerdings erhöhen 
auch die Schwangerschaften mit in vitro gezeugten Einlingen diese Komplikationen und die damit verbundenen Spätfolgen signifikant. Zudem führt die Befruchtung der Eizellen in vitro zu einer Erhöhung der Fehlbildungsrate. Schliesslich wird auch ein Zusammenhang zwischen Befruchtung in vitro sowie Krebs und beeinträchtigter kognitiver Entwicklung im Kindesalter diskutiert.

Wie werden diese Risiken durch die Technologie beeinflusst?

Zu den Risiken für die Kinder durch die Kryokonservierung der Eizellen gibt es zurzeit nur wenige Studien (vgl. nächste Frage). Bisher wurden vor allem die gesundheitlichen Risiken für die Kinder in Zusammenhang mit der ovariellen Stimulation, der Entnahme und der Befruchtung der Eizellen in vitro untersucht. Eine rasch wachsende Zahl von neueren Untersuchungen zeigt, dass neben anderen möglichen Einflüssen, wie zum Beispiel elterlicher Subfertilität und mütterlichem Alter, die reproduktionsmedizinische Technologie selbst signifikante Risiken mit sich bringt. Insbesondere häufen sich in den letzten Jahren die Hinweise, dass durch die reproduktionsmedizinischen Verfahren ausgelöste epigenetische Veränderungen die erhöhten gesundheitlichen Risiken bei in vitro gezeugten Kindern erklären. Solche epigenetischen Veränderungen betreffen Schaltmechanismen, die Gene ein- oder ausschalten, ohne die Gene selbst zu verändern. Da gewisse dieser Schaltmechanismen insbesondere bei der Bildung der Eizellen oder vor der Einnistung des Embryos in der Eizelle aktiviert respektive deaktiviert werden, ist es relativ naheliegend, dass die ovarielle Stimulation, die Eizellentnahme, die Manipulierung der Spermien oder die für die Ausreifung der Embryonen benötigten Nährmedien zu epigenetischen Veränderungen führen könnten. Relativ gut belegt ist, dass die reproduktionsmedizinischen Verfahren zu epigenetischen Veränderungen führen, die in Zusammenhang mit seltenen Krankheiten wie dem Beckwith-Wiedemann-Syndrom (BWS) und dem Angelman-Syndrom (AS) stehen. Verschiedene epidemiologische Studien und Tierversuchsmodelle liefern zudem klare Hinweise, dass die durch die reproduktionsmedizinischen Verfahren ausgelösten epigenetischen Veränderungen zu vorzeitiger Blutgefässalterung, Lungenarteriendysfunktion, Bluthochdruck und Herzdysfunktionen führen können. Diese Ergebnisse sind noch relativ neu und müssen bestätigt werden, sie wurden aber bisher auch nicht durch vergleichbare Untersuchungen widerlegt. Falls sie sich in Zukunft bestätigen, würde eine Ausdehnung der assistierten Reproduktion auf grössere Bevölkerungsschichten durch Social Freezing bedeutsame Auswirkungen auf die 
öffentliche Gesundheit haben. Weitere Studien sind insbesondere notwendig, um zu klären, ob die epigenetischen Veränderungen auf die Technologie, die Subfertilität der Eltern oder eine Kombination von beidem zurückzuführen sind. Unbedenklich wären reproduktionsmedizinische Verfahren aus sozialen Gründen nur dann, wenn die damit verbundenen gesundheitlichen Risiken für die Kinder ausschliesslich auf die Subfertilität der Eltern zurückzuführen wären.

Wie lange können die kryokonservierten Eizellen aufbewahrt werden und welche Folgen hat die Konservierung für die daraus entstandenen Kinder?

Es gibt nur einzelne Studien zur Lagerungsdauer der Eizellen. Es wurden bereits Kinder aus Eizellen geboren, die zwölf Jahre gelagert worden waren. Entscheidender als die Lagerungsdauer scheint für den reproduktionsmedizinischen Erfolg der Einfrierungs- und Auftauprozess zu sein. Auch zu den Folgen der Konservierung der Eizellen für die daraus entstandenen Kinder bestehen noch wenige Daten. Die Fehlbildungsrate bei Kindern aus eingefrorenen Eizellen scheint nicht höher zu sein als diejenige bei Kindern aus frischen Eizellen. Allerdings stammen die Daten entweder aus Eizellspendeprogrammen oder von unfruchtbaren Paaren. Es kann nicht beurteilt werden, ob sich diese Ergebnisse auf Frauen übertragen lassen, die ihre Eizellen aus sozialen Gründen kryokonservieren lassen.

Wie werden die gesundheitlichen Risiken für das Kind durch das Alter der Frau beeinflusst?

Mit zunehmendem Alter der Frau steigt das Risiko von Komplikationen in der Schwangerschaft (Schwangerschaftsdiabetes, Bluthochdruck, Schwangerschaftsvergiftung) und bei der Geburt (Kaiserschnitt). Kinder von älteren Müttern werden früher geboren und haben ein tieferes Geburtsgewicht als Kinder von jüngeren Müttern. Diese Risiken steigen ab 35 Jahren exponentiell und sind ab Mitte 40 und insbesondere ab Anfang 50 beträchtlich. 
Wie beeinflussen die Kryokonservierung und die entsprechende Erfahrung der Fertilitätsklinik die Erfolgsaussichten?

In Kliniken mit langjähriger Expertise unterscheiden sich die Befruchtungs- und Schwangerschaftsraten nach der Befruchtung von eingefrorenen Eizellen in vitro kaum mehr von denjenigen mit frischen Eizellen. Dieser Befund stützt sich insbesondere auf vier randomisierte, kontrollierte Studien einzelner Kliniken. Zwei davon wurden in Spanien, gestützt auf Daten aus Eizellspendeprogrammen, und zwei in Italien mit Daten von unfruchtbaren Paaren realisiert. Beobachtungsstudien in Italien mittels Daten einer grossen Brandbreite von Kliniken mit unterschiedlicher Expertise gelangen zu weniger positiven Ergebnissen. Es bestehen zudem Zweifel, ob die Daten von jungen fruchtbaren Eizellspenderinnen auf ältere Frauen übertragen werden können, die zurzeit ihre Eizellen aus sozialen Gründen kryokonservieren lassen.

Wie beeinflusst das Alter der Frauen die Erfolgsaussichten?

Da bisher nur wenige Frauen auf ihre eingefrorenen Eizellen zurückgegriffen haben, kann die Erfolgsaussicht dieser Technologie noch nicht beurteilt werden. In vier Studien wurde jedoch die theoretische Geburtenchance berechnet. Diese Studien zeigen, dass die Aussicht auf ein Kind mit zunehmendem Alter der Frau bei der Eizellentnahme sinkt. Da sich die Berechnung der theoretischen Geburtenchance in den verschiedenen Studien auf unterschiedliche klinische Daten, Patientenkollektive und Berechnungsmethoden stützte, kann jedoch nicht abschliessend eingeschätzt werden, bis zu welchem Alter und mit wie vielen eingefrorenen Eizellen Social Freezing Erfolg versprechend ist. Bei Frauen unter 35 Jahren wird die Chance auf ein Kind mit 60 bis 80 Prozent angegeben, wenn 15 bis 20 Eizellen eingefroren worden sind. Dafür sind in der Regel ein bis zwei Behandlungszyklen notwendig. Bei über 35-Jährigen ist Social Freezing nur Erfolg versprechend, wenn noch genügend und qualitativ gute Eizellen vorhanden sind. 
Welche Aspekte sollten im medizinischen Beratungsgespräch behandelt werden, um eine informierte Entscheidung für oder gegen Social Freezing sicherzustellen?

Zu den Inhalten des Beratungsgesprächs liegen Angaben von acht der 30 online befragten Fertilitätskliniken vor. In den meisten dieser acht Kliniken werden die Kosten, der Ablauf der Behandlung, die individuellen Erfolgsaussichten, die Risiken für Eizellen, Mutter und Kind sowie die rechtlichen Rahmenbedingungen bezüglich Aufbewahrungsfrist und Vernichtung der Eizellen systematisch erläutert und vertraglich geregelt. Nur in wenigen Fällen wird allerdings die Erfahrung der Klinik in der Anwendung der Technologie thematisiert. Zudem kann nicht ausgeschlossen werden, dass die Frauen ungenügend über die gesundheitlichen Risiken für das Kind informiert werden. Während eine interviewte Frau wissenschaftliche Studien dazu im Internet gesucht und sich darüber mit ihrem Arzt unterhalten hat, hat eine andere Frau nicht aktiv nach solchen Informationen gesucht und wurde auch kaum über allfällige Risiken informiert. Die interviewten Reproduktionsmediziner informieren zurückhaltend über allfällige epigenetische Risiken der Technologie, weil die Evidenz noch nicht breit abgestützt ist und sie die Frauen nicht verunsichern wollen. Da es sich nur um einzelne Aussagen handelt, können diese Erkenntnisse nicht verallgemeinert und andere Motive nicht ausgeschlossen werden. Aus einer ethischen Perspektive ist allerdings zu betonen, dass eine informierte Entscheidung eine wichtige Voraussetzung für die Ausübung der Fortpflanzungsfreiheit ist. Je weniger dringend ein Eingriff medizinisch ist, desto höheren Anforderungen muss die Information genügen. Eine umfassende Information wird von den 23 interviewten gesellschaftlichen Akteuren einvernehmlich als äusserst wichtig erachtet. Informationen zu den Risiken für das Kind sind zudem für die im Rahmen der Studie befragten 408 kinderlosen Frauen nach den Kosten das wichtigste Kriterium, um über eine Inanspruchnahme der Technologie zu entscheiden.

\section{1 .2}

\section{Aktuelle Nachfrage und künftiges Interesse am Social Freezing}

In diesem Kapitel wird die Nachfrage nach Social Freezing, gestützt auf wissenschaftliche Artikel, explorative Interviews mit Reproduktionsmedizinern und 
Frauen sowie eine Umfrage bei potenziell interessierten kinderlosen Frauen zwischen 25 und 40 Jahren, beschrieben.

Wie viele Frauen nehmen in der Schweiz aktuell Social Freezing in Anspruch? Wie viele reisen dafür ins Ausland?

Gemäss einer groben Schätzung, gestützt auf eine Umfrage bei Reproduktionsmedizinern, haben im Jahr 2017 in der Schweiz rund 400 Frauen ihre Eizellen einfrieren lassen. Die Nachfrage ist in den letzten Jahren kontinuierlich gewachsen. In einer der Kliniken hat sich die Nachfrage beispielweise zwischen 2014 und 2017 vervierfacht. Auch künftig wird ein weiteres Wachstum der Nachfrage erwartet. Zur Anzahl Frauen, die ins Ausland reisen, liegen keine Angaben vor. Ausschlaggebend dürften primär die rechtlichen Rahmenbedingungen und nicht die Kosten sein. Eine interviewte Frau hat sich in Spanien über Social Freezing informiert, es dann aber aus praktischen Gründen vorgezogen, die Behandlung in der Schweiz vornehmen zu lassen. Für sie war es wichtig, dass sie nach Ablauf der Lagerungsfrist von zehn Jahren ihre Eizellen ins Ausland transferieren kann.

Aus welchen Gründen nehmen Frauen Social Freezing in Anspruch? Welche Erwartungen haben Frauen an die Technologie?

Sowohl im Ausland als auch in der Schweiz sind Frauen, die ihre Eizellen kryokonservieren lassen, mehrheitlich über 35 Jahre alt, alleinstehend und gut ausgebildet. Eine Minderheit leidet unter einer medizinisch begründeten Unfruchtbarkeit (z.B. Endiometriose, polyzystisches Ovarialsyndrom, verfrühte Menopause). Im Gegensatz zu den gängigen Vorstellungen in der Öffentlichkeit weisen die befragten Frauen der Vereinbarkeit von Karriere und Familie eine untergeordnete Rolle bei ihrem Entscheid für Social Freezing zu. Sowohl in der Literatur als auch von den zwei interviewten Schweizer Frauen wird als wichtigster Grund für die Inanspruchnahme dieser Technologie das Fehlen eines geeigneten Partners genannt. Als geeignet werden Männer erachtet, die dem Bild des «neuen Vaters» entsprechen. Diese Väter nehmen gemeinsam mit den Müttern die Verantwortung für die Kindererziehung wahr und möchten sich intensiv in der Kinderbetreuung engagieren. 
Die Frauen verknüpfen essenziell drei Erwartungen mit der Technologie des Social Freezing:

- Erstens wird die Technologie als eine Möglichkeit gesehen, die traditionelle und naturalisierte Familienbeziehung aufrechtzuerhalten. Im Gegensatz zur Eizellspende oder Adoption gewährleistet Social Freezing eine genetische Verbindung zwischen Mutter und Kind. Einzelne Studien zeigen darüber hinaus, dass auch die Sicherstellung einer genetischen Verbindung zum Vater und zu den Grosseltern als Vorteil des Social Freezing gesehen wird.

- Zweitens erwarten die Frauen, dass Social Freezing innen erlaubt, den sozialen und psychischen Zeithorizont mit der biologischen Uhr in Einklang zu bringen und zum richtigen Zeitpunkt Mutter zu werden. Der richtige Zeitpunkt wird durch biografische, von gesellschaftlichen Normen geprägte Voraussetzungen bestimmt. Eine gute Mutterschaft wird einerseits mit sozialen Voraussetzungen wie einer gesicherten Stelle, einem guten Einkommen und einer angemessenen Wohnsituation in Verbindung gebracht. Andererseits soll eine gute Mutter die notwendigen psychischen Voraussetzungen mitbringen und sich intensiv ihrem Kind widmen können.

- Drittens wird Social Freezing von den Frauen als eine Art Versicherung gegen die Unfruchtbarkeit gesehen, die innen das Gefühl vermittelt, alles Machbare gegen eine drohende Kinderlosigkeit unternommen zu haben.

\section{Wiegt Social Freezing Frauen in falscher Sicherheit?}

Die internationalen Studien deuten darauf hin, dass bei den meist über 35jährigen Frauen zu wenig Eizellen entnommen wurden, um gute Chancen auf eine Lebendgeburt zu erzielen. Es gibt Hinweise, dass sich die Frauen dieser Einschränkungen bewusst sind. So hätten einige gerne mehr Eizellen entnommen respektive die Entnahme in jüngerem Alter vorgenommen. Auch in der Schweiz scheinen nicht immer genügend Eizellen entnommen zu werden. Es ist allerdings unklar, ob sich die Frauen in falscher Sicherheit wiegen. 
Wie, wann und unter welchen Voraussetzungen wollen Frauen später auf ihre kryokonservierten Eizellen zurückgreifen?

Bisher haben maximal ein Zehntel der Frauen auf ihre eingefrorenen Eizellen zurückgegriffen. Dies dürfte einerseits auf die kurze Zeitspanne zwischen Eizellentnahme und der Befragung der Frauen in den Studien zurückzuführen sein. Andererseits streben die meisten Frauen selbst in hohem Alter zuerst eine natürliche Zeugung mit einem Partner an. Einige Frauen sind auf diesem Weg respektive über eine Befruchtung frisch entnommener Eizellen in vitro schwanger geworden. Die Befruchtung der eingefrorenen Eizellen mittels einer Spermienspende wird erst als letzte Option in Betracht gezogen. Gemäss internationalen Studien erachten es maximal ein Drittel der Frauen als sehr wahrscheinlich, dass sie ihre kryokonservierten Eizellen in vitro befruchten lassen. Zwei Drittel würden die Eizellen nur unter bestimmten Umständen nutzen.

Im europäischen Umfeld scheinen die Frauen spätestens mit 50 Jahren Mutter werden zu wollen, während in den USA für eine grosse Mehrheit der Frauen auch eine Mutterschaft über 50 Jahren gut vorstellbar ist. Auch die befragten Frauen in der Schweiz möchten mehrheitlich vor Mitte 40 Mutter werden. Lediglich 5 Prozent könnten sich eine Mutterschaft über 45 vorstellen.

Es gibt Hinweise, dass die Frauen ihre nicht verwendeten kryokonservierten Eizellen primär der Forschung zur Verfügung stellen würden. Nur eine Minderheit kann sich eine Eizellspende vorstellen. In der Schweiz konnte sich eine interviewte Frau eine Eizellspende an eine andere Frau nur nach ihrem Tode vorstellen, eine andere Frau konnte es sich überhaupt nicht vorstellen.

Wie gross ist das potenzielle Interesse an dieser Technologie in Zukunft?

Social Freezing ist in der Schweiz noch nicht sehr bekannt. Die Onlinebefragung bei 408 kinderlosen Frauen zwischen 25 und 39 Jahren zeigt, dass nur knapp die Hälfte der Befragten (48 \%) schon einmal von Social Freezing gehört hat. In der Westschweiz haben deutlich weniger Befragte Kenntnis von der Technologie als in der Deutschschweiz. Der Vergleich mit anderen Ländern, in denen entsprechende Umfrageergebnisse vorliegen, weist darauf hin, dass der Kenntnisstand zum Social Freezing in der Schweiz relativ tief ist. 
Ohne nähere Informationen zur Technologie erhalten zu haben, können 40 Prozent der Befragten sich vorstellen, ihre Eizellen einfrieren zu lassen, oder haben dies bereits getan ( $1 \%$ ); 7 Prozent wissen es nicht oder machen keine Angaben bei dieser Frage, die restlichen 52 Prozent können es sich nicht vorstellen. Ein Anteil von 40 Prozent an Interessierten ist etwas höher als im internationalen Vergleich, allerdings sind die Ergebnisse nicht direkt vergleichbar, weil die Stichprobenzusammensetzung der befragten Frauen bezüglich Alter und Lebenssituation (kinderlos/mit Kind) variiert. Nachdem die Frauen detaillierte Zusatzinformationen zum Verfahren, zu den Risiken für Mutter und Kind, den Erfolgsaussichten, den Kosten sowie zur Rechtslage erhalten haben, beträgt der Anteil der Frauen, die sich vorstellen könnten, die Eizellen einfrieren zu lassen, noch 34 Prozent. 57 Prozent können sich dies nach detaillierten Informationen nicht mehr vorstellen und 9 Prozent wissen es nicht oder beantworten die Frage nicht. Am stärksten negativ beeinflusst werden die potenziellen Nutzerinnen dabei durch nähere Informationen zu den Kosten sowie den Risiken für die Kinder. Am geringsten ist die negative Beeinflussung durch Informationen zum Verfahren.

Gestützt auf diese Ergebnisse der Befragung, wird das Potenzial an Frauen, die künftig ihre Eizellen vorsorglich entnehmen und einfrieren lassen, eingeschätzt:

- Das hohe Szenario umfasst jene 34 Prozent der Befragten, die sich nach dem Erhalt der Detailinformationen zur Technologie grundsätzlich vorstellen könnten, ihre Eizellen vorsorglich einfrieren zu lassen. Basierend auf Angaben des BFS zur Anzahl kinderloser Frauen zwischen 25 und 39 in der Schweiz, würde das Potenzial demnach maximal bei 150'000 Frauen liegen, die im Zeitraum zwischen 25 und 39 Jahren ihre Eizellen einmal einfrieren lassen würden. Pro Jahr wären dies knapp 10'000 Frauen. In der Realität dürften wesentlich weniger Befragte ein Social Freezing in Erwägung ziehen, weil sich ihre Lebensumstände ändern oder sie noch detailliertere Informationen davon abhalten würden.

- Ein mittleres Szenario geht daher von den 7 Prozent kinderloser Frauen zwischen 25 und 39 aus, die sich vorstellen können, Social Freezing zu beanspruchen und in der Befragung von keiner Detailinformation zur Technologie negativ in ihrem Entscheid beeinflusst wurden. Das Potenzial würde in diesem Szenario 30'800 Frauen über den gesamten Beobachtungszeitraum und rund 2000 Frauen pro Jahr umfassen, die bereit wären, 
ihre Eizellen einfrieren zu lassen. Diese 7 Prozent entsprechen auch in etwa den Ergebnissen aus internationalen Studien und beschreiben jenen Anteil an Frauen, bei denen sich nicht nur ein grundsätzliches, sondern ein konkretes Interesse am Social Freezing manifestiert.

- Geht man nur von jenen Frauen aus, die gemäss Befragung Social Freezing bereits in Anspruch genommen haben, so ist dies 1 Prozent. Dieses tiefe Szenario, das ein Potenzial von 4400 Frauen über den gesamten Beobachtungszeitraum und knapp $\mathbf{3 0 0}$ Frauen pro Jahr bedeuten würde, dürfte für ein künftiges Potenzial zu tief sein, weil die Kenntnis über Social Freezing in der Schweiz im Vergleich zu ausländischen Studien noch relativ gering ist und davon auszugehen ist, dass das Interesse am Social Freezing mit steigender Bekanntheit auch noch zunehmen wird.

Wie viele Kinder letztlich jährlich aufgrund dieser Szenarien mittels Social Freezing geboren werden, kann nur sehr grob eingeschätzt werden. Vom Einfrieren der Eizellen bis zur Geburt eines Kindes ist es ein langer Weg. Geht man davon aus, dass etwa 20 Prozent der Frauen mit kryokonservierten Eizellen diese später in vitro befruchten lassen, und nimmt man weiter an, dass von diesen Frauen die Hälfte ein Kind zur Welt bringt, so würden einst gemäss mittlerem Szenario in der Schweiz jährlich rund 200 Kinder mittels Social Freezing geboren werden. Diese Zahl ist allerdings mit grossen Unsicherheiten behaftet und könnte sich aufgrund neuer technologischer und rechtlicher Rahmenbedingungen ändern.

\subsubsection{Ethische Folgen des Social Freezing}

Die Informationen in diesem Kapitel beruhen auf Artikeln in der wissenschaftlichen Literatur und in den Schweizer Medien.

Zudem wird in diesem Kapitel die Haltung zum Social Freezing von drei Meinungstypen beschrieben. Die drei Meinungstypen wurden durch ein Clustering der Antworten von 23 befragten Schweizer Akteuren aus verschiedenen Parteien und Fachgesellschaften gebildet. Der ablehnende Meinungstyp ist gegen Social Freezing. Ausschlaggebend sind in erster Linie ethische Bedenken, insbesondere in Zusammenhang mit dem Kindeswohl. Der ambivalente Meinungs- 
typ ist grundsätzlich nicht gegen Social Freezing im Einzelfall, hat aber gesellschaftliche Bedenken gegen die Technologie und möchte die Frauen vor gesellschaftlichen Zwängen sowie ökonomischen Interessen der Anbieter der Technik schützen. Der befürwortende Meinungstyp vertritt eine liberale Haltung und steht Social Freezing grundsätzlich offen gegenüber.

Die 23 befragten Akteure stammen aus verschiedenen politischen Parteien, Kirchen und Fachorganisationen. Da in den meisten Organisationen keine fortgeschrittene Meinungsbildung zum Social Freezing stattgefunden hat, gaben die befragten Akteure ihre subjektive Sichtweise auf das Thema wieder. Beim Social Freezing handelt es sich um ein sehr persönliches Thema, das stark von subjektiven Faktoren wie Alter, Geschlecht und dem eigenen unerfüllten Kinderwunsch geprägt wird.

Reicht es, wenn Frauen oder Paare eine gut informierte Entscheidung treffen, oder ist es Aufgabe der Gesellschaft oder des Staats, die Rahmenbedingungen wie den Verwendungszweck von Eizellen oder das Höchstalter der Frau festzulegen?

Sowohl in der wissenschaftlichen Literatur als auch in den Schweizer Medien wird die Fortpflanzungsfreiheit als wichtiges Gut angesehen. Für Verfechtende eines liberalen Autonomieverständnisses in der Literatur reicht eine ausführliche Information als Entscheidungsgrundlage für Social Freezing. Kritikerinnen und Kritiker des Social Freezing berufen sich dagegen auf ein relationales Autonomiekonzept. Die Inanspruchnahme des Social Freezing wird als Folge gesellschaftlicher Zwänge gesehen. Entsprechend wird eine ausführliche Information der Frauen als ungenügend erachtet, um einen autonomen Entscheid für Social Freezing zu ermöglichen. Die Kritikerinnen und Kritiker plädieren zwar für eine gesellschaftliche statt einer technologischen Lösung dieser Zwänge. Daraus leiten sie aber keinen Regulierungsbedarf für diese Technologie ab. Ein Regulierungsbedarf ergibt sich primär, wenn die Fortpflanzungsfreiheit mit dem Kindeswohl (vgl. nachfolgende Frage) und gesamtgesellschaftlichen Interessen in Konflikt steht. Die gesellschaftsethische Diskussion fokussiert sich vor allem auf die Chancengleichheit der Geschlechter insbesondere in Zusammenhang mit der Vereinbarkeit von Beruf und Familie (vgl. Kapitel 9.1.4). Andere gesellschaftliche Interessen wie die Auswirkungen einer breiten Anwendung des Social Freezing auf die öffentliche Gesundheit waren nicht Gegenstand der 
Diskussion in der internationalen Literatur oder in den Schweizer Medien. Gemäss der Nationalen Ethikkommission im Bereich der Humanmedizin kann es eine Aufgabe des demokratisch legitimierten Gesetzgebers sein, gesellschaftsethische Fragen zu klären und Rahmenbedingungen beispielsweise zum Höchstalter für die Befruchtung in vitro zu setzen.

Die im Rahmen der Studie befragten Schweizer Akteure sind einheitlich der Meinung, dass ein informierter Entscheid eine wichtige Voraussetzung für die Ausübung der Fortpflanzungsfreiheit ist. Allerdings sind Vertretende des ablehnenden und des ambivalenten Meinungstyps der Ansicht, dass Social Freezing nicht nur ein Ausdruck eines autonomen Entscheides, sondern auch von gesellschaftlichen Zwängen ist. Die Vertretenden des befürwortenden Meinungstyps teilen diese Ansicht überhaupt nicht. Für den befürwortenden und den ambivalenten Meinungstyp gibt es keine guten Gründe, einer gut informierten Frau die Möglichkeit des Social Freezing zu verwehren, nur weil keine medizinische Notwendigkeit vorliegt. Für den ablehnenden Typ stellt die fehlende medizinische Indikation einen Grund dar, Social Freezing einzuschränken.

Wie muss damit umgegangen werden, wenn Fortpflanzungsfreiheit und Kindeswohl in Konflikt stehen? Was ist höher zu werten und warum?

Es ist in der Literatur und den Schweizer Medien unbestritten, dass eine Einschränkung der Fortpflanzungsfreiheit zur Schadensvermeidung bei Dritten zulässig ist. In diesem Sinne ist das Kindeswohl grundsätzlich über die Fortpflanzungsfreiheit zu stellen. Kontrovers diskutiert wird allerdings, ob Kinder durch Social Freezing tatsächlich einen Schaden erleiden. Im Fokus der Diskussion steht dabei das fortgeschrittene Alter der Frau bei der Befruchtung der Eizellen in vitro und bei der anschliessenden Schwangerschaft (vgl. nachfolgende Frage). Die Auswirkungen der Technologie werden seltener diskutiert. Allfällige gesundheitliche Risiken für die Kinder aufgrund epigenetischer Veränderungen in Zusammenhang mit ovarieller Stimulation und Befruchtung der Eizellen in vitro sind selten Gegenstand der Diskussion. In der Regel werden mögliche Auswirkungen der Kryokonservierung der Eizellen auf die Kinder diskutiert und wird auf die beruhigende, wenn auch dünne Datenlage verwiesen. Aus diesem Grund wird, gestützt auf das Vorsorgeprinzip, eine zurückhaltende Anwendung der Kryokonservierung vorgeschlagen. Andere argumentieren hingegen, dass das Kindeswohl nicht in dem Ausmass gefährdet ist, dass eine 
Einschränkung der Fortpflanzungsfreiheit gerechtfertigt wäre. Falls die Risiken dieser Technologien für das Kind zu hoch wären, müsste auch unfruchtbaren Paaren und Krebspatientinnen der Zugang zu diesen Technologien verboten werden. Diese Argumentation blendet allerdings aus, dass bei einer allgemeinen Einschränkung der Fortpflanzungsfreiheit durchaus Ausnahmen für besonders benachteiligte Paare möglich sind.

Die befragten 23 Schweizer Akteure sind in dieser Frage gespalten. Für Vertretende des ablehnenden Meinungstyps besteht die Gefahr, dass bei Social Freezing das Kindeswohl gegenüber den elterlichen Wünschen in den Hintergrund tritt. Weil zu wenig Studien zu den gesundheitlichen Folgen der Eizellkonservierung für die Kinder bestehen, soll die Technik nicht breit angewendet werden dürfen. Vertretende des ambivalenten und des befürwortenden Meinungstyps sind mit diesen Einschätzungen tendenziell nicht einverstanden.

Wie ist das Kindeswohl in Bezug auf die physische und psychische Entwicklung zu beurteilen, wenn ein grosser Altersunterschied zwischen Mutter und Kind besteht? Welche ethischen Konsequenzen ergeben sich daraus?

Eine Mutterschaft in fortgeschrittenem Alter wird in der internationalen Literatur ablehnend beurteilt, weil sie mit erhöhten Schwangerschaftsrisiken einhergeht und zu einer frühen Verwaisung mit traumatischen Folgen für das Kind führen kann. Demgegenüber wird argumentiert, dass eine späte Mutterschaft bessere soziale und psychische Voraussetzungen für eine erfolgreiche Kindererziehung mit sich bringt, wie stabilere Familienverhältnisse und eine grössere finanzielle Sicherheit. Es wird auf Studien verwiesen, die eine bessere sprachliche Entwicklung der Kinder und eine geringere Anzahl von Unfällen sowie Krankenhauseinweisungen aufzeigen. Allerdings sind die entsprechenden Studien schwer interpretierbar, weil ein hochkomplexes Netzwerk von Einflussfaktoren besteht.

Gibt es ein Recht auf ein Kind in jedem Lebensalter?

Diese Frage wird sowohl in der Literatur als auch in den Schweizer Medien kontrovers diskutiert. Um die Risiken in Zusammenhang mit Schwangerschaft und Verwaisung infolge einer späten Mutterschaft zu reduzieren, sprechen sich 
einige Autorinnen und Autoren für eine Alterslimite aus. Sie argumentieren, dass es sich bei der Fortpflanzungsfreiheit um ein negatives und nicht um ein positives Recht handelt. Der Staat darf die Fortpflanzung zwar nicht einschränken, er ist aber auch nicht verpflichtet, den Zugang zu reproduktionsmedizinischen Verfahren in jedem Alter sicherzustellen. Andere argumentieren hingegen, dass eine Alterslimite abzulehnen ist, weil es keine solche Grenze für Männer gibt, obwohl auch bei diesen die gesundheitlichen Risiken für den Nachwuchs mit zunehmendem Alter des Vaters steigen. Aus diesem Grund wird eine Einschränkung des Rechts auf ein Kind in jedem Lebensalter nur dann als zulässig angesehen, wenn sie für beide Geschlechter gilt. Die Nationale Ethikkommission stellt sich auf den Standpunkt, dass eine solche sozialethische Diskussion nur durch den demokratisch legitimierten Gesetzgeber entschieden werden kann. Die Beurteilung medizinischer Risiken sei im Rahmen von Richtlinien medizinischer Fachgesellschaften vorzunehmen.

Bei den befragten Schweizer Akteuren sprechen sich insbesondere die Vertretenden des ablehnenden und des befürwortenden Meinungstyps für eine Alterslimite aus. Kritisch gegenüber einer fixen Alterslimite äussert sich der ambivalente Meinungstyp. Die Vertretenden des ambivalenten Typs befürworten eher eine Beurteilung des individuellen Risikos durch die behandelnden Ärztinnen und Ärzte. Ausschlaggebendes Argument für eine Alterslimite sind für die Mehrheit der befragten Akteure die medizinischen Risiken. Sozialethische Überlegungen spielen eine untergeordnete Rolle. Die Befragten sind mehrheitlich der Ansicht, dass keine pauschalen Aussagen beispielsweise zur finanziellen Sicherheit oder zur Energie von älteren Müttern in der Kinderbetreuung gemacht werden können, sondern dass es hier grosse interindividuelle Unterschiede gibt. Einzig bei der Vorgabe des Fortpflanzungsmedizingesetzes, wonach Eltern ihre Kinder bis zur Volljährigkeit begleiten müssen, zeigen sich Unterschiede. Während der ablehnende Meinungstyp bezweifelt, dass diese Vorgabe beim Social Freezing eingehalten werden kann, erkennen die anderen beiden Meinungstypen keine solche Gefahr. Wenig Gewicht haben Argumente, welche die Notwendigkeit einer gleichen Alterslimite für beide Geschlechter betreffen. 
Was soll mit den überzähligen eingefrorenen Eizellen passieren?

Die Frage der Verwendung überzähliger Eizellen wird in den Schweizer Medien nicht und in der Literatur nur vereinzelt diskutiert. Bisher haben noch sehr wenige Frauen auf ihre eingefrorenen Eizellen zurückgegriffen. Da Frauen in der Regel zuerst eine Schwangerschaft auf natürlichem Weg anstreben, dürfte Social Freezing zu überzähligen Eizellen führen. Drei Verwendungszwecke stehen dafür im Vordergrund: die Vernichtung, die Spende der Eizellen für die Forschung oder eine Eizellspende an unfruchtbare Frauen. Bei einer Eizellspende zur Behandlung von Unfruchtbarkeit oder zu Forschungszwecken müssten die Eigentumsrechte an den Eizellspenden klar geregelt werden. In Ländern mit Eizellspende scheinen die Eigentumsrechte zurzeit unklar. Einerseits könnten die Frauen die Rechte an den kryokonservierten Eizellen bis zu deren Verwendung behalten. Andererseits könnten diese Rechte auch an die Eizellbank respektive die Abnehmerinnen und Abnehmer der Eizellen übergehen. Dadurch könnten diese ohne Zustimmung der Eizellspenderin über eine allfällige Weitervermittlung der Eizellen entscheiden.

Sind eine rein vorsorgliche Eizellentnahme und die anschliessende Vernichtung ethisch vertretbar?

Diese Frage wird von der European Society of Human Reproduction and Embryology (ESHR) aufgeworfen, aber nicht weiter diskutiert. Die Eizellen stellen im Gegensatz zu Embryonen weder ethisch noch rechtlich ein schutzwürdiges Gut dar. Es ist entsprechend davon auszugehen, dass keine ethischen Einwände gegen die Vernichtung überzähliger Eizellen bestehen. Die im Rahmen der Studie befragten Schweizer Akteure haben zu dieser Frage keine klare Haltung.

Sollte es möglich sein, entnommene Eizellen für eine allfällige Spende oder die Forschung zur Verfügung zu stellen?

Diese Frage wird in der Literatur relativ selten diskutiert. Befürworterinnen und Befürworter dieser Möglichkeit schlagen vor, dass die Forschenden einen Beitrag an die Kosten des Social Freezing leisten könnten und im Gegenzug einen Teil der entnommenen Eizellen erhalten. Dies dürfte das Angebot an raren Eizellen für die Forschung erhöhen. Die Frauen scheinen eher bereit, ihre Eizel- 
len der Forschung statt einer unfruchtbaren Frau zur Verfügung zu stellen. Sie sind so nicht mit der Vorstellung konfrontiert, dass irgendwo ein Kind von innen aufwächst. Zudem müsste die Frau in diesem Rahmen nicht lediglich für Forschungszwecke die aufwendige und mit medizinischen Risiken verbundene Behandlung zur Eizellentnahme auf sich nehmen. Allerdings gibt es auch Befürchtungen, dass Social Freezing eine Strategie ist, um das Verbot der Entschädigung von Eizellspenden zu Forschungszwecken zu unterlaufen. Es besteht die Gefahr, dass ein Schwarzmarkt für Eizellen entsteht und die Frauen ausgebeutet werden. Darum wird vereinzelt gefordert, dass der Beitrag der Frauen an die Wertschöpfung der Forschung nicht nur finanziell anerkannt wird, sondern dass die Anforderungen an einen informierten Entscheid, die Sicherheit des Verfahrens und die Nachbetreuung klar geregelt werden.

Die Meinungen bezüglich einer Eizellspende für die Forschung sind bei den befragten 23 Schweizer Akteuren gespalten. Während für den ablehnenden Meinungstyp ethische Bedenken gegenüber einer Spende überzähliger Eizellen an die Forschung im Fokus stehen, ist der befürwortende Meinungstyp offen für eine Spende für die Forschung. Der ambivalente Meinungstyp vertritt keine klare Haltung in dieser Frage.

Sollen entnommene Eizellen für eine allfällige Eizellspende zur Verfügung gestellt werden?

In der Schweiz wird sich diese Frage nur stellen, wenn das Verbot der Eizellspende aufgehoben wird. Für die ethische Beurteilung dieser Frage ist es wichtig, zu welchem Zeitpunkt die Eizellspende erfolgt. Im Rahmen eines «Freeze and Share»-Arrangements werden die Kosten der Eizellkonservierung bei unter 35-jährigen Frauen übernommen, wenn diese im Gegenzug die Hälfte ihrer Eizellen spenden. Es ist möglich, dass die Spenderin nie auf ihre eigenen Eizellen zurückgreifen wird, während die Empfängerin dank der gespendeten Eizellen Mutter wird. Diese Situation kann für die Spenderin psychisch belastend sein. Solche Bedenken sind allerdings hinfällig, wenn die Frau ihre überzähligen Eizellen erst spendet, nachdem sie Mutter geworden ist oder mit dem Kinderwunsch abgeschlossen hat. Die psychologischen Folgen einer Eizellspende sind in einer solchen Situation besser abschätzbar. Weiter wird befürchtet, dass mit den «Freeze and Share»-Arrangements eine Kommerzialisierung der Eizellen einhergeht, die auf einer asymmetrischen Beziehung zwischen reichen in- 
fertilen Frauen und armen fruchtbaren Frauen beruht. Andere argumentieren hingegen, dass «Freeze and Share» für Frauen mit geringen Mitteln die einzige Option ist, ihren Kinderwunsch weiterleben zu lassen.

Auch bezüglich der Eizellspende an unfruchtbare Frauen haben die für die Studie befragten politischen und gesellschaftlichen Akteure eine unterschiedliche Haltung. Der ablehnende Meinungstyp spricht sich klar gegen eine solche Eizellspende aus, weil er eine ethisch bedenkliche Kommerzialisierung der Eizellen befürchtet. Die anderen beiden Meinungstypen befürworten eine solche Spende an unfruchtbare Frauen, die sich im Gegenzug an den Kosten des Social Freezing beteiligen. Damit wird ein Beitrag geleistet, um die soziale Ungleichheit im Zugang zum Social Freezing zwischen gut und schlecht verdienenden Frauen zu reduzieren.

Stellt Social Freezing eine akzeptablere Alternative zur Eizellspende dar?

Die Vermeidung einer Eizellspende dient als wichtigste Rechtfertigung des Social Freezing. Im Vergleich zur Eizellspende sind die ethischen Bedenken gegenüber Social Freezing geringer, weil keine finanzielle Notlage der Spenderin ausgenutzt wird. Im Gegensatz zur Eizellspende ist beim Social Freezing zudem die genetische Verbindung zwischen Mutter und Kind sichergestellt, wodurch psychosoziale Komplikationen vermieden werden können. Ausserdem sind die Schwangerschaftskomplikationen, insbesondere Schwangerschaftsvergiftungen, bei einer Eizellspende höher als bei einer Schwangerschaft mit eigenen Eizellen. Schliesslich sorgt Social Freezing dafür, dass die Nachfrage nach den raren Spendereizellen abnimmt. Es gibt allerdings auch Stimmen, die befürchten, dass Social Freezing nicht als Alternative zur Eizellspende zu sehen ist, sondern diese im Gegenteil weiter ankurbelt. Weil dank Social Freeing mehr Eizellen gelagert werden, könnten spezialisierte Eizellbanken entstehen. Diese könnten die Lagerung und den Vertrieb der Eizellen effizienter organisieren als die Anbieter fortpflanzungsmedizinischer Verfahren. Dadurch könnten die Kosten für die Eizellspende gesenkt und die Nachfrage gesteigert werden.

Die befragten Schweizer Akteure sind mehrheitlich der Ansicht, dass Social Freezing eine akzeptablere Alternative zur Eizellspende darstellt. Insbesondere die genetische Verbindung zwischen Mutter und Kind scheint für den befürwortenden und den ambivalenten Meinungstyp ausschlaggebend. Für Vertretende 
des ambivalenten Meinungstyps ist zudem von Bedeutung, dass sich die Frau selber dem aufwendigen Verfahren unterzieht und nicht eine fremde unbeteiligte Eizellspenderin. Weil der ablehnende Meinungstyp sowohl der Eizellspende als auch Social Freezing kritisch gegenübersteht, lässt sich zu dieser Frage keine klare Haltung erkennen.

\subsubsection{Gesellschaftliche Folgen des Social Freezing}

Die Informationen in diesem Kapitel beruhen auf Artikeln in der wissenschaftlichen Literatur und in den Schweizer Medien. Zudem werden die Antworten der 23 befragten Schweizer Akteure aufgeführt (vgl. Kapitel 9.1.3).

Wie wirkt sich Social Freezing auf die gesellschaftlichen Bemühungen zur Vereinbarkeit von Beruf und Familie sowie zur Förderung der Chancengleichheit der Geschlechter aus?

Das Zusammenspiel zwischen Social Freezing und der Vereinbarkeit von Beruf und Familie wird in der wissenschaftlichen Literatur und in den Schweizer Medien rege diskutiert. Befürworterinnen und Befürworter der Technologie sind der Ansicht, dass Social Freezing einen Beitrag leistet, um die auf biologische Unterschiede zurückzuführende Ungleichheit von Männern und Frauen zu reduzieren und die Chancengleichheit der Geschlechter zu fördern. Insbesondere in den Schweizer Medien überwiegen allerdings die kritischen Stimmen. Es wird befürchtet, dass die Frauen wegen Social Freezing ihre Familienplanung zugunsten ihrer Arbeitstätigkeit zurückstellen. Statt gesellschaftliche Strukturen zur Förderung der Vereinbarkeit von Beruf und Familie auszubauen, wird die Lösung gesellschaftlicher Zwänge der einzelnen Frau überlassen. Die Befürworterinnen und Befürworter des Social Freezing in der Literatur bezweifeln allerdings, dass Social Freezing anstelle von Massnahmen zur Förderung von Beruf und Familie treten wird. Massnahmen auf individueller und gesellschaftlicher Ebene werden nicht als antagonistisch, sondern als komplementär erachtet. Diese Einstellung vertritt auch die Nationale Ethikkommission in ihrer Stellungnahme zum Social Freezing. 
Mit der ausführlichen Diskussion des Zusammenspiels von Social Freezing sowie der Vereinbarkeit von Beruf und Familie wird dem Thema eine Bedeutung beigemessen, die durch die Aussagen von den befragten Frauen und Reproduktionsmedizinern nicht gestützt wird. Ausschlaggebend für die Inanspruchnahme des Social Freezing sind nicht primär Karriereüberlegungen, sondern der fehlende passende Partner. Dieser Sachverhalt wird allerdings in den wissenschaftlichen Beiträgen nur anekdotisch diskutiert. Es fehlt sowohl bei den Fürsprechern als auch bei den Kritikerinnen und Kritikern des Social Freezing eine umfassende und systematische Reflexion über das Zusammenspiel von Social Freezing und den Familiengründungsabsichten von Männern und Frauen. Die Hauptursache für dieses Versäumnis dürfte sein, dass Social Freezing, aber auch die Familiengründung generell in erster Linie als Angelegenheit der Frauen wahrgenommen und diskutiert werden. Mehrere Exponentinnen und Exponenten in den Schweizer Medien kritisieren jedoch den Umstand, dass Social Freezing die Hauptverantwortung für die Familienplanung bei den Frauen belässt. Gemäss einer Studie suchen Frauen mit kryokonservierten Eizellen einen Partner, der sich stark in der Kindererziehung engagiert und die Kinderbetreuung gemeinsam mit der Mutter wahrnimmt. Gefragt wären entsprechend nicht nur Massnahmen für Frauen, welche die Arbeitstätigkeit von Müttern begünstigen, sondern auch solche für Väter, die ihnen ein stärkeres Engagement in der Familie erlauben.

Die für die Studie befragten Schweizer Akteure erkennen im Social Freezing mehrheitlich kein emanzipatorisches Potenzial. Im Gegenteil, die Vertretenden des ablehnenden und insbesondere des ambivalenten Meinungstyps befürchten, dass sich Social Freezing negativ auf die Bemühungen zur Vereinbarkeit von Beruf und Familie sowie die Chancengleichheit der Geschlechter auswirkt. Der befürwortende Meinungstyp teilt diese Befürchtungen nicht.

Inwiefern leistet Social Freezing einer Medikalisierung sozialer Probleme Vorschub?

Der Vorwurf, dass Social Freezing einer Medikalisierung gesellschaftlicher Probleme Vorschub leistet, wird vor allem in der wissenschaftlichen Literatur rege diskutiert. Einige Autorinnen und Autoren argumentieren, dass Social Freezing einer Medikalisierung sozialer Probleme klar Vorschub leiste. Im Rahmen des Social Freezing wird, im Gegensatz zu regulären reproduktions- 
medizinischen Verfahren, nicht nur die Infertilität als medizinisches Problem definiert, sondern bereits die Abnahme der Fertilität. Gleichzeitig werden medizinische Gründe vorgebracht, um gerechtfertigte von therapeutisch nicht gerechtfertigten Interventionen zu unterscheiden und die Ausweitung von medizinischen Lösungen für gesellschaftliche Probleme zu unterbinden. So hob die amerikanische Gesellschaft für Reproduktionsmedizin im Jahr 2012 zwar den experimentellen Status für die Eizellkonservierung auf. Sie empfahl jedoch, diese Technologie nur bei medizinischen, nicht aber bei sozialen Gründen anzuwenden. Diese Unterscheidung ist allerdings umstritten. So werden im Bereich der Fortpflanzung verschiedene Eingriffe wie Verhütung, Sterilisation, Abtreibung, In-vitro-Befruchtung bei älteren Paaren vorgenommen, die primär aus sozialen Gründen erfolgen. Zudem wird argumentiert, dass Social Freezing durchaus einen therapeutischen Nutzen hat. Gute Medizin umfasse nicht nur die Behandlung, sondern auch die Prävention von Krankheiten. Die Antizipation einer ungewollten Infertilität wird in diesem Sinne als präventive Medizin gesehen. Die Bezeichnung einer altersbedingten Unfruchtbarkeit als Krankheit kann allerdings wiederum als Medikalisierung sozialer Probleme gesehen werden. Zudem könnte eine breite Anwendung des Social Freezing zu einer bedeutsamen Zunahme nicht übertragbarer Krankheiten (NCD) führen, falls sich die deutliche Erhöhung des damit verbundenen Risikos von Herz-KreislaufErkrankungen bestätigen würde.

Die Meinungen der befragten Schweizer Akteure sind in dieser Frage gespalten. Die Vertretenden des ablehnenden und des ambivalenten Meinungstyps stehen der Anwendung medizinischer Lösungen der Fortpflanzungsmedizin für soziale Probleme kritisch gegenüber. Der befürwortende Meinungstyp ist jedoch klar dagegen, dass man die Anwendung von medizinischen Lösungen der Fortpflanzungsmedizin für soziale Probleme unterbindet.

Wie würde sich eine Zunahme des Social Freezing auf die Familienbilder in der Gesellschaft auswirken?

Diese Frage wird in der Literatur nur am Rand und in den Schweizer Medien praktisch nicht diskutiert. Der Einfluss des Social Freezing auf die Familienbilder wird in der Literatur als widersprüchlich erachtet. So begünstigt Social Freezing durch die hohe Wertschätzung der genetischen Verbindung zwischen beiden Elternteilen und dem Kind ein traditionelles naturalistisches Familienbild. 
Gleichzeitig wird ein solches Familienbild durch eine Mutterschaft jenseits der biologischen Altersgrenze infrage gestellt. Social Freezing wirkt sich aber auch auf die erweiterte Familie aus und kann eine stark auseinanderklaffende Generationenabfolge begünstigen. Dies kann einerseits dazu führen, dass die Kinder mehrheitlich in einem Familienverbund mit lediglich zwei Generationen aufwachsen. Andererseits wird auch die Pflegearbeit zwischen Generationen beeinträchtigt. Die Grosseltern können die Mütter nicht bei der Kindererziehung entlasten. Sie sorgen im Gegenteil durch ihre eigene Pflegebedürftigkeit für eine Doppelbelastung der Mütter, die sich gleichzeitig um ihre kleinen Kinder und ihre betagten Eltern kümmern müssen. Dieses Schicksal dürfte auch die aus kryokonservierten Eizellen gezeugten Kinder betreffen. Gleichzeitig können sie sich die Pflegearbeit ihrer Eltern möglicherweise nicht mit Geschwistern teilen. Wenn, wie empfohlen, nur 15 bis 20 Eizellen eingefroren werden, kann voraussichtlich lediglich eine Lebendgeburt erzielt werden.

Auch bei den im Rahmen dieser Studie befragten Schweizer Akteuren scheint der Einfluss des Social Freezing auf die Familienbilder oder intergenerationelle Beziehungen (z.B. Pflegearbeit) nicht von zentraler Bedeutung zu sein. Bei Vertretenden des ambivalenten und des ablehnenden Meinungstyps gibt es leichte Bedenken, dass die durch Social Freezing gezeugten Kinder ihre Grosseltern nur noch im Pflegeheim oder gar nicht mehr erleben.

Wie gross ist die Akzeptanz des Social Freezing insgesamt bei Interessengruppen? Wie werden die Chancen und Risiken des Social Freezing von Interessengruppen eingeschätzt und beurteilt?

Die Befragung der Schweizer Akteure aus verschiedenen politischen Parteien, Kirchen und Fachorganisationen zeigt, dass in der Schweiz ein breites Meinungsspektrum zum Social Freezing besteht. Beim Social Freezing handelt es sich um ein sehr persönliches Thema, das stark von subjektiven Faktoren wie Alter, Geschlecht und dem eigenen Kinderwunsch geprägt wird. Während ein Verbot des Social Freezing für die Mehrheit der befragten Akteure keine Option darstellt, existieren doch viele Bedenken und Unsicherheiten rund um die Anwendung dieser Technik. Die wichtigen Konsenspunkte betreffen den Informationsbedarf der Frauen, die sich für Social Freezing interessieren. Wenig Bedeutung messen alle Akteure dem Einfluss des Social Freezing auf Familienbilder und intergenerationelle Beziehungen zu. Konfliktpunkte betreffen die Verwendung der Eizellen, Risiken fürs Kindeswohl, die Notwendigkeit einer 
Alterslimite, die Verlängerung der Aufbewahrungsfrist, der Einfluss des Social Freezing auf die Vereinbarkeit von Beruf und Familie sowie die Kostenübernahme durch die Krankenversicherung.

\subsubsection{Finanzielle Folgen des Social Freezing}

Die Informationen in diesem Kapitel beruhen auf Artikeln in der wissenschaftlichen Literatur und in den Schweizer Medien. Zudem werden die Antworten der 23 befragten Schweizer Akteure aufgeführt (vgl. 9.1.3).

\section{Wer hat welche ökonomischen Interessen am Social Freezing?}

Frauen, die ihre Eizellen kryokonservieren lassen, investieren relativ viel Geld in ein Verfahren, dessen Erfolgschancen zum jetzigen Zeitpunkt nicht klar bestimmt werden können. Viele Autorinnen und Autoren in der wissenschaftlichen Literatur und einzelne Leserinnen und Leser von Schweizer Medien fragen sich darum, ob Social Freezing wirklich einem Bedürfnis dieser Frauen entspricht oder ob ihre Nachfrage nicht eher durch die Anbieter dieser Technologie erzeugt wurde. Einzelne Autorinnen und Autoren empfinden den Vorwurf, dass die Frauen durch die Anbieter beeinflusst werden, als bevormundend und sexistisch.

Aus der Gesundheitsökonomie können zwei Konzepte herangezogen werden, um den Zusammenhang zwischen Social Freezing und den kommerziellen Interessen der Anbieter zu diskutieren. So könnte beim Social Freezing einerseits eine angebotsinduzierte Nachfrage spielen. Eine solche angebotsinduzierte Nachfrage entsteht durch ein Informationsgefälle zwischen Arzt und Patient, was dem Arzt erlaubt, die Nachfrage nach seinen Leistungen zu seinem ökonomischen Vorteil zu beeinflussen. Je mehr Ärztinnen und Ärzte eine medizinische Leistung erbringen, desto häufiger wird diese auch bei fraglicher oder fehlender Indikation angewendet. In der Literatur konnte keine Studie identifiziert werden, die eine solche angebotsinduzierte Nachfrage beim Social Freezing untersucht. Die empirischen Untersuchungen im Rahmen dieser Studie deuten allerdings darauf hin, dass in den Fertilitätskliniken durchaus Kapazitäten für eine Leistungsausweitung mittels Social Freezing vorhanden sind. 
Ebenso gibt es einzelne Hinweise, dass die Reproduktionsmedizinerinnen und -mediziner eher zurückhaltend über die Risiken der Technologie informieren. Andererseits spielen beim Social Freezing keine Fehlanreize, die durch die Übernahme der Kosten von medizinischen Leistungen durch die Krankenkassen entstehen (moral hazard). Die Frauen kommen für die effektiven Kosten des Social Freezing auf und müssen nicht, wie bei kassenpflichtigen Leistungen, lediglich die Versicherungsprämie begleichen. Sie haben entsprechend wenig finanzielle Anreize, mehr Leistungen nachzufragen als notwendig. Die Kosten sind auch der wichtigste Faktor, der den im Rahmen dieser Studie befragten potenziell interessierten Frauen die Inanspruchnahme des Social Freezing erschweren würde.

Die für diese Studie befragten Schweizer Akteure sind geteilter Meinung, ob die Anbieter des Social Freezing einen Einfluss auf die Nachfrage haben. Der befürwortende Meinungstyp ist relativ klar der Meinung, dass die Nachfrage nicht durch die Anbieter künstlich gesteigert wird und daher Frauen auch nicht vor den ökonomischen Interessen der Anbieter geschützt werden müssen. Die beiden anderen Typen sprechen sich jedoch für einen solchen Schutz der Frauen aus.

Können sich Unternehmen eine Kostenbeteiligung vorstellen und wie würde diese beurteilt?

Aus drei Gründen ist es nicht sehr wahrscheinlich, dass sich Unternehmen in der Schweiz eine Kostenbeteiligung am Social Freezing vorstellen können. Erstens bleibt die Beteiligung von Facebook und Apple an den Kosten des Social Freezing auch in den USA eine Randerscheinung und dürfte keine breite Anwendung ausserhalb dieses sehr technologieaffinen Umfelds finden. Zweitens ist die Kostenübernahme des Social Freezing in den USA vor dem Hintergrund des dortigen Gesundheitswesens zu verstehen. Amerikanische Unternehmen schliessen für ihre Angestellten eine Krankenversicherung ab und definieren den Leistungskatalog. In der Schweiz haben die Arbeitgeber keine solche Rolle und die Kostenübernahme würde eher im Rahmen des Krankenversicherungsgesetzes erfolgen. Drittens wird die Beteiligung von Arbeitgebern an den Kosten des Social Freezing sowohl in der Literatur als auch in den Schweizer Medien weitgehend negativ beurteilt. Es wird befürchtet, dass der 
Druck auf Frauen steigt, die Familiengründung zugunsten ihrer Karriere zurückzustellen.

Bei den befragten Schweizer Akteuren sprechen sich die Vertretenden des ambivalenten Meinungstyps deutlich gegen eine Kostenbeteiligung durch Unternehmen aus. Während der ablehnende Typ keine klare Haltung in dieser Frage hat, erkennt der befürwortende Meinungstyp überhaupt keine Gefahr bei der Kostenbeteiligung durch Unternehmen.

Sollen die Kosten von den Frauen oder von den Krankenkassen übernommen werden?

Dieses Thema wird sowohl in der Literatur als auch in den Schweizer Medien nur am Rande diskutiert. In der Schweiz stellt sich diese Frage nur, wenn sich die Krankenkassen an den Kosten für die Befruchtung der Eizellen in vitro beteiligen. Relativ unbestritten scheint allerdings, dass die Kosten für die Eizellentnahme und -konservierung auch in Zukunft von den Frauen beglichen werden sollten, weil die künftige Nutzung der eingefrorenen Eizellen ungewiss ist. Hingegen sollen für die Befruchtung der eingefrorenen Eizellen in vitro im Hinblick auf die Chancengleichheit im Zugang zur Reproduktionsmedizin sowie die Kosteneffizienz die gleichen finanziellen Bedingungen gelten wie für andere Patientinnen.

Bei den befragten Schweizer Akteuren sprechen sich die ablehnende und die ambivalente Gruppe klar gegen eine Kostenbeteiligung der Krankenkassen aus. Für die ablehnende Gruppe ist vor allem ausschlaggebend, dass Social Freezing medizinisch nicht notwendig ist. Die liberale Gruppe könnte sich auch keine Übernahme der Kosten der Eizellentnahme und -konservierung vorstellen, jedoch eine Beteiligung an den Kosten der Befruchtung der Eizellen in vitro, um auch weniger gut verdienenden Frauen einen Zugang zum Social Freezing zu ermöglichen. 
Wie wirken sich die Kosten des Social Freezing auf die Inanspruchnahme dieser Technologie aus?

Sowohl die Angaben in der Literatur als auch die Aussagen der Schweizer Reproduktionsmediziner sowie der befragten Frauen deuten darauf hin, dass Social Freezing im Moment ausschliesslich von gut verdienenden Frauen in Anspruch genommen wird. Der Einfluss der Kosten auf die Inanspruchnahme des Social Freezing zeigt sich auch in der Befragung der kinderlosen Frauen zwischen 25 und 39 Jahren im Rahmen dieser Studie. Von den 408 befragten Frauen gaben 59 Prozent an, dass die Informationen zu den Kosten in der Umfrage es innen erschweren würde, Social Freezing zu beanspruchen. Die Kosten des Social Freezing wirken sich auch negativer auf das Interesse an dieser Technologie aus als etwa die Risiken für Mutter und Kind.

\subsubsection{Rechtliche Beurteilung des Social Freezing}

Die nachfolgend beschriebenen Ergebnisse stützen sich hauptsächlich auf die Rechtsvergleichung in Kapitel 8. Daneben werden auch die Ergebnisse aus der Befragung der Reproduktionsmedizinerinnen und -mediziner (vgl. Kapitel 2.4) und der 23 gesellschaftlichen Akteure (vgl. Kapitel 7) punktuell miteinbezogen.

Wie ist Social Freezing in der Schweiz zurzeit gesetzlich geregelt in Bezug auf Indikation, soziale Kriterien, Aufbewahrungsfrist sowie Alterslimite?

In der Schweiz ist Social Freezing nicht explizit geregelt. In der Literatur geht man davon aus, dass die Entnahme der Eizellen nicht dem Fortpflanzungsmedizingesetz unterliegt und entsprechend ohne medizinische Indikation zulässig ist. Bei der In-vitro-Befruchtung der Eizellen braucht es allerdings eine medizinische Indikation, wozu unter anderem Unfruchtbarkeit gehört. Ob darunter auch die altersbedingte Unfruchtbarkeit fällt, ist in der Literatur umstritten. Ohne entsprechende Klärung durch den Gesetzgeber fehlt es jedoch an einer Grundlage, um einer altersbedingten unfruchtbaren Frau den Zugang zu ihren kryokonservierten Eizellen zu verwehren. Zudem lässt sich meist nicht klären, ob die Unfruchtbarkeit auch andere Gründe als das fortschreitende Alter hat. Für die kryokonservierten Eizellen gilt eine Aufbewahrungsfrist von fünf Jahren, 
welche einmalig und ohne nähere Begründung um weitere fünf Jahre auf maximal zehn Jahre verlängert werden kann. Die Befruchtung der kryokonservierten Eizellen ist nur zulässig, wenn die Frau in einer stabilen heterosexuellen Partnerschaft lebt. Sowohl für die Entnahme als auch die In-vitro-Befruchtung der Eizellen gibt es keine fixe Alterslimite. Allerdings muss nach dem Gesetzestext bei der Anwendung fortpflanzungsmedizinischer Verfahren stets das Kindeswohl gewährleistet sein. Daraus ergibt sich auch eine «soziale» Altersgrenze, weil Fortpflanzungsverfahren nur bei Paaren angewendet werden dürfen, die aufgrund ihres Alters und ihrer persönlichen Verhältnisse voraussichtlich bis zur Volljährigkeit des Kindes für dessen Pflege und Erziehung sorgen können.

\section{Wie unterscheidet sich diese Regelung von anderen Ländern?}

Die Rechtsvergleichung zeigt, dass die Rechtslage bezüglich Social Freezing in Europa ausgesprochen uneinheitlich ist. Während Spanien, Tschechien und Belgien sehr liberale Regelungen haben, ist Social Freezing in Österreich verboten. Ebenfalls eine restriktive Regelung kennt Frankreich. Dort ist Social Freezing nur in Verknüpfung mit einer Eizellspende zulässig. Diese gesetzliche Vorgabe ist nicht nur eine grosse Hürde für Frauen, die Bedenken gegen eine Eizellspende haben, sondern ist auch mit einem beträchtlichen Aufwand für die interessierten Frauen verbunden. So ist eine Kryokonservierung der Eizellen zum Eigengebrauch erst dann möglich, wenn mehr als fünf reife Eizellen gespendet werden können. Um 15 bis 20 Eizellen für den Eigengebrauch kryokonservieren zu können, müssten mehrere Stimulationszyklen durchlaufen werden und rund 30 bis 40 Eizellen entnommen werden können. Zwischenstellungen nehmen Deutschland und Italien ein. In Deutschland gibt es keine Regelung und in Italien stützt sich die Gesetzgebung teilweise auf regionale Bestimmungen. Die Unterschiede zwischen den Ländern lassen sich nicht vollständig mit dem Alter der jeweiligen Gesetzgebung erklären. Es lässt sich also nicht etwa sagen, dass neuste Gesetzesrevisionen liberaler sind. Die wichtigsten Gemeinsamkeiten und Unterschiede in der Gesetzgebung der sieben untersuchten Ländern und der Schweiz sind in Tabelle 5 aufgeführt.

- Zulässigkeit der Entnahme und der Kryokonservierung der Eizellen: Mit Ausnahme von Frankreich und Österreich ist die Entnahme und die Kryokonservierung von Eizellen in allen Ländern zulässig. Explizit erlaubt sind Eizellentnahme und Kryokonservierung aus sozialen Gründen jedoch nur 
in Belgien. In den übrigen Ländern fehlt eine explizite Regelung. Wie in der Schweiz sind auch in Italien die Eizellentnahme und die Kryokonservierung nicht der Fortpflanzungsmedizingesetzgebung unterstellt. In Deutschland besteht aufgrund des fehlenden Fortpflanzungsmedizingesetzes überhaupt keine Regelung. In den Gesetzgebungen von Spanien und der Tschechischen Republik wird nicht zwischen medizinischen und sozialen Gründen unterschieden.

- Altersgrenze für die Entnahme und die Kryokonservierung der Eizellen: Wie die Schweiz auch kennen die meisten Länder keine Alterslimite für die Entnahme und Konservierung der Eizellen. In Belgien müssen die Frauen volljährig sein und können die Eizellen bis spätestens einen Tag vor ihrem 46. Geburtstag kryokonservieren lassen. Frankreich kennt eine Altersgrenze von 18 bis 38 Jahren in Zusammenhang mit der Eizellspende.

- Zulässigkeit der Befruchtung der Eizellen in vitro: In den meisten Ländern ist die In-vitro-Befruchtung der aus sozialen Gründen kryokonservierten Eizellen zulässig. Während es in Spanien, Belgien und der Tschechischen Republik keine medizinische Indikation braucht, muss bei den Frauen in der Schweiz und Italien eine medizinisch bestätigte Unfruchtbarkeit vorliegen. Diese kann allerdings auch altersbedingt sein. Im Gegensatz dazu erlaubt Frankreich eine Befruchtung der Eizellen in vitro nur dann, wenn die Unfruchtbarkeit pathologischer Natur ist. Bei einer physiologischen Unfruchtbarkeit, worunter auch die altersbedingte Unfruchtbarkeit der Frau fällt, ist die Befruchtung der Eizellen in vitro verboten.

- Altersgrenze für die Befruchtung der Eizellen in vitro: Im Gegensatz zur Schweiz kennen die meisten Länder eine Altersgrenze für die Befruchtung der Eizellen in vitro. In Belgien gilt eine Altersgrenze von 47 und in der Tschechischen Republik von 49 Jahren. In Italien ist die Altersgrenze regional recht uneinheitlich geregelt, aber aus verfassungsrechtlichen Gründen ist vom 50. Altersjahr auszugehen. Spanien kennt zwar keine gesetzliche Regelung, aber eine Empfehlung der Klinikverbände, die eine Behandlung bis zum 50. Altersjahr vorsieht. In Frankreich gibt es keine fixe Altersgrenze, allerdings muss die Frau noch im fruchtbaren Alter sein. In der Praxis hat sich eine relativ tiefe Altersgrenze von 42 bis 43 Jahren etabliert. Kein anderes Land beruft sich wie die Schweiz ausschliesslich auf eine soziale Altersgrenze. So müssen Schweizer Eltern ihr Kind nach der In-vitro- 
Befruchtung bis zur Volljährigkeit begleiten können. In Italien verlangt die Gesetzgebung zwar auch, dass die Eltern noch in der Lage sind, sich um die Bedürfnisse ihres Kindes zu kümmern. Gleichzeitig sollen auch Risiken in Zusammenhang mit einer Schwangerschaft im fortgeschrittenen Alter beachtet werden.

- Aufbewahrungsfrist: Die meisten untersuchten Länder kennen keine Aufbewahrungsfrist für die Lagerung der Eizellen. Lediglich in Belgien besteht wie in der Schweiz eine Lagerungsdauer von zehn Jahren. Allerdings kann in Belgien diese Lagerungsdauer im Ermessen der Fertilitätskliniken jederzeit verlängert werden.

\section{Tabelle 5: $\quad$ Übersicht über die Rechtslage in acht Ländern}

\begin{tabular}{|c|c|c|c|c|c|}
\hline Land & Eizellentnahme & $\begin{array}{l}\text { Kryo- } \\
\text { konservierung } \\
\text { Eizellen }\end{array}$ & $\begin{array}{l}\text { Befruchtung } \\
\text { Eizellen in } \\
\text { vitro }\end{array}$ & $\begin{array}{l}\text { Aufbewah- } \\
\text { rungsfrist }\end{array}$ & $\begin{array}{l}\text { Informa- } \\
\text { tionspflicht }\end{array}$ \\
\hline Österreich & \multicolumn{5}{|c|}{$\begin{array}{l}\text { Social Freezing ist verboten; fortpflanzungsmedizinische Massnahmen sind nur bei } \\
\text { medizinischer Indikation erlaubt. }\end{array}$} \\
\hline $\begin{array}{l}\text { Frank- } \\
\text { reich }\end{array}$ & $\begin{array}{l}\text { Zulässig, sofern } \\
\text { Eizellspende } \\
18 \text { bis } 38 \text { Jahre } \\
\text { für Eizellspende }\end{array}$ & $\begin{array}{l}\text { Zulässig, sofern } \\
\text { Eizellspende } \\
18 \text { bis } 38 \text { Jahre } \\
\text { für Eizellspende }\end{array}$ & $\begin{array}{l}\text { Zulässig } \\
18 \text { bis ca. } \\
42 / 43 \text { Jahre }\end{array}$ & Nein & $\mathrm{Ja}$ \\
\hline $\begin{array}{l}\text { Deutsch- } \\
\text { land }\end{array}$ & $\begin{array}{l}\text { Zulässig } \\
\text { Keine Alters- } \\
\text { grenze }\end{array}$ & $\begin{array}{l}\text { Zulässig } \\
\text { Keine Alters- } \\
\text { grenze }\end{array}$ & $\begin{array}{l}\text { Zulässig } \\
\text { Keine Alters- } \\
\text { grenze }\end{array}$ & Nein & $\mathrm{Ja}$ \\
\hline Italien & $\begin{array}{l}\text { Zulässig } \\
\text { Keine Alters- } \\
\text { grenze }\end{array}$ & $\begin{array}{l}\text { Zulässig } \\
\text { Keine Alters- } \\
\text { grenze }\end{array}$ & $\begin{array}{l}\text { Zulässig } \\
50 . \text { Altersjahr }\end{array}$ & Nein & $\mathrm{Ja}$ \\
\hline Belgien & $\begin{array}{l}\text { Zulässig } \\
18 \text { bis } 45 \text { Jahre }\end{array}$ & $\begin{array}{l}\text { Zulässig } \\
18 \text { bis } 45 \text { Jahre }\end{array}$ & $\begin{array}{l}\text { Zulässig } \\
18 \text { bis } \\
47 \text { Jahre }\end{array}$ & $\begin{array}{l}10 \text { Jahre, } \\
\text { Verlänge- } \\
\text { rung im Er- } \\
\text { messen der } \\
\text { Klinik }\end{array}$ & $\mathrm{Ja}$ \\
\hline Spanien & $\begin{array}{l}\text { Zulässig } \\
\text { Keine Alters- } \\
\text { grenze }\end{array}$ & $\begin{array}{l}\text { Zulässig } \\
\text { Keine Alters- } \\
\text { grenze }\end{array}$ & $\begin{array}{l}\text { Zulässig } \\
\text { Keine Alters- } \\
\text { grenze }\end{array}$ & Nein & $\mathrm{Ja}$ \\
\hline $\begin{array}{l}\text { Tschechi- } \\
\text { sche } \\
\text { Republik }\end{array}$ & $\begin{array}{l}\text { Zulässig } \\
\text { Keine Alters- } \\
\text { grenze }\end{array}$ & $\begin{array}{l}\text { Zulässig } \\
\text { Keine Alters- } \\
\text { grenze }\end{array}$ & $\begin{array}{l}\text { Zulässig } \\
\text { 49. Altersjahr }\end{array}$ & Nein & Nein \\
\hline Schweiz & $\begin{array}{l}\text { Zulässig } \\
\text { Keine Alters- } \\
\text { grenze }\end{array}$ & $\begin{array}{l}\text { Zulässig } \\
\text { Keine Alters- } \\
\text { grenze }\end{array}$ & $\begin{array}{l}\text { Zulässig } \\
\text { Keine Alters- } \\
\text { grenze }\end{array}$ & 10 Jahre & Nein \\
\hline
\end{tabular}


- Informationspflicht: Weil die Entnahme und die Kryokonservierung der Eizellen in der Schweiz nicht dem Fortpflanzungsmedizingesetz unterstellt sind, sind die Fertilitätskliniken auch nicht verpflichtet, sich an die Informations- und Beratungspflicht zu halten (Art. 6 FMedG). Mit Ausnahme der Tschechischen Republik kennen alle anderen Länder eine mehr oder weniger umfassende Informationspflicht. In Deutschland dürfte das Patientenrechtegesetz zur Anwendung kommen. Dieses orientiert sich am Grundsatz, dass die Aufklärung umso umfangreicher sein soll, je weniger dringend ein Eingriff medizinisch indiziert ist.

Inwiefern besteht ein Reformbedarf? Sollte statt der Aufbewahrungsfrist für die Eizellen eine Alterslimite für die Frauen festgelegt werden?

Für die aktuelle Aufbewahrungsdauer von maximal zehn Jahren gibt es keine medizinischen Gründe. Nach dem aktuellen Stand der Medizin und Technik bleiben kryokonservierte Eizellen längere Zeit in gleichbleibend gutem Zustand. Da zurzeit die meisten Frauen bei der Kryokonservierung ihrer Eizellen bereits 35 Jahre alt sind und in der Regel vor ihrem 45. Geburtstag auf ihre Eizellen zurückgreifen möchten, reicht eine Lagerungsfrist von zehn Jahren in den meisten Fällen. Da die Qualität der Eizellen mit zunehmendem Alter abnimmt, wären allerdings eine Eizellentnahme weit vor dem 35. Geburtstag und eine längere Aufbewahrungsfrist sinnvoll. Als umständlich wird in der Praxis auch die Pflicht empfunden, nach fünf Jahren eine Verlängerung der Frist auf zehn Jahre zu beantragen. Alle befragten Reproduktionsmedizinerinnen und -mediziner sprechen sich für eine Verlängerung der Aufbewahrungsdauer aus. Eine Mehrheit kann sich auch eine Aufhebung der Aufbewahrungsfrist zugunsten einer Alterslimite vorstellen. Dies würde auch eher der Praxis in den meisten untersuchten europäischen Ländern entsprechen. Bei den befragten Schweizer Akteuren sind die Meinungen in dieser Frage gespalten. Während sich die ambivalente und die befürwortende Meinungsgruppe für eine Verlängerung der Aufbewahrungsfrist aussprechen, ist die ablehnende Meinungsgruppe dagegen. Ausschlaggebend dürfte sein, dass sich diese Meinungsgruppe generell keine Lockerung der rechtlichen Rahmenbedingungen für Social Freezing wünscht. Bezüglich der Frage der «Altersgrenze statt Aufbewahrungsfrist» scheint die Meinungsfindung in allen drei Gruppen nicht eindeutig respektive noch nicht abgeschlossen zu sein. Eine interviewte Frau, die ihre Eizellen einfrieren liess, stösst sich an der Alterslimite. Sie ist der Ansicht, dass nur sie über ihre eige- 
nen Eizellen verfügen kann. Sie würde diese nach Ablauf der Aufbewahrungsfrist ins Ausland transferieren. Die Altersgrenze wäre hingegen nur für eine kleine Minderheit der online befragten potenziell interessierten Frauen ein Grund, sich gegen Social Freezing zu entscheiden.

Wenn ja, welche Alterslimite ist aus medizinischer, ethischer, gesellschaftlicher und rechtlicher Sicht angemessen?

Unter Einbezug all dieser Perspektiven dürfte sich am ehesten ein Alter von 45 Jahren als Grenze anbieten. Aus medizinischer Sicht steigt ab 35 Jahren das Risiko von Schwangerschafts- und Geburtskomplikationen mit negativen Folgen für Mutter und Kind exponentiell an und ist ab 45 beträchtlich. An einer Altersgrenze von 45 Jahren scheinen sich auch die online befragten Schweizer Fertilitätszentren zu orientieren. Die Spannbreite bewegt sich zwischen 43 und 48 Jahren, wobei in den meisten befragten Kliniken eine Altersgrenze von 45 Jahren für eine In-vitro-Befruchtung mit kryokonservierten Eizellen gilt.

Aus einer individualethischen Perspektive ist eine Alterslimite vor allem in Zusammenhang mit dem Kindeswohl von Bedeutung. Da die Risiken für die Kinder bei einem mütterlichen Alter von über 45 Jahren zunehmen, scheint auch aus einer ethischen Perspektive eine Einschränkung der Fortpflanzungsfreiheit in diesem Alter zulässig, zumal es sich bei der Fortpflanzungsfreiheit nicht um ein positives Recht handelt mit einer Verpflichtung für den Staat, den Zugang zu reproduktionsmedizinischen Verfahren in jedem Alter sicherzustellen.

Aus einer gesellschaftsethischen Perspektive ist insbesondere die Chancengleichheit der Geschlechter hervorzuheben. Eine Altersgrenze von 45 Jahren, die für Frauen, nicht aber für Männer gilt, ist unter diesem Gesichtspunkt schwer zu rechtfertigen. Für den ambivalenten Meinungstyp ist dies ein Argument gegen eine fixe Alterslimite. Grundsätzlich sprechen aus Sicht der befragten Schweizer Akteure jedoch in erster Linie die medizinischen Risiken für eine Altersgrenze bei der In-vitro-Befruchtung. Gesellschaftsethische Überlegungen wie die Chancengleichheit der Geschlechter im Zugang zur Reproduktionsmedizin, die Ressourcen älterer Mütter in der Kindererziehung sowie der Einfluss des Social Freezing auf Familienbilder und Generationenbeziehungen spielen eine untergeordnete Rolle. 
Aus rechtlicher Sicht ist zu betonen, dass sich die anderen europäischen Länder in der Regel auf eine medizinisch gestützte Alterslimite stützen und die soziale Altersgrenze im schweizerischen Fortpflanzungsmedizingesetz international eine Ausnahme darstellt. Allerdings ist für die Festlegung einer Altersgrenze nicht eine Angleichung der schweizerischen an eine ausländische Rechtsordnung ausschlaggebend. Vielmehr sollte eine Regelung geschaffen werden, die den innerstaatlichen Wertungen möglichst gut entspricht. Dabei drängt sich auf, die Überlegungen, die sich der Gesetzgeber in Zusammenhang mit dem neuen Adoptionsrecht gemacht hat, für fortpflanzungsmedizinische Verfahren zu bedenken. Dazu gehört die im seit Anfang 2018 geltenden Adoptionsrecht festgesetzte Altersgrenze. Diese beruht auf einem maximalen Altersunterschied zwischen Adoptivkind und Adoptiveltern von 45 Jahren. Davon kann zwar aus Gründen des Kindeswohls im Einzelfall abgewichen werden. In Zusammenhang mit fortpflanzungsmedizinischen Verfahren ist allerdings gerade nicht auf das Wohl eines bereits lebenden, auf «neue» Eltern angewiesenen Kindes Rücksicht zu nehmen. Es ist daher nicht einzusehen, weshalb das neue Adoptionsrecht eine konkrete Altersgrenze vorsieht, das Fortpflanzungsmedizinrecht hingegen nicht.

Soll die In-vitro-Befruchtung der Eizelle weiterhin nur heterosexuellen Paaren mit diagnostizierter medizinischer Unfruchtbarkeit vorbehalten bleiben oder sollten diese rechtlichen Voraussetzungen gelockert werden?

Diese Frage wurde nicht umfassend untersucht. In Frankreich, Italien und der Tschechischen Republik haben nach den derzeit (Frühjahr 2018) geltenden Regelungen lediglich heterosexuelle Paare Zugang zur Reproduktionsmedizin. Spanien hat dagegen eine sehr liberale Gesetzgebung. Urteilsfähige Frauen haben ungeachtet ihrer sexuellen Orientierung Zugang zur Reproduktionsmedizin. In Belgien können reproduktionsmedizinische Verfahren auch von alleinstehenden Frauen in Anspruch genommen werden. In Deutschland fehlt es an einem Fortpflanzungsmedizingesetz, weshalb die Behandlung lesbischer Paare mit Spendersamen grundsätzlich - vorbehältlich anderslautender standesrechtlicher Regeln - zulässig ist und in wenigen Kliniken auch praktiziert wird. Interessant ist zudem die Gesetzgebung von Österreich, wo allerdings Social Freezing gerade nicht zugelassen ist. Im Rahmen einer Revision des österreichischen Fortpflanzungsmedizingesetzes im Jahr 2015 haben Frauenpaare Zugang zur Reproduktionsmedizin erhalten. Dem vorangegangen war eine 
Entscheidung des österreichischen Verfassungsgerichtshofes vom 10. Dezember 2013, der unter Berufung auf das Diskriminierungsverbot in der Europäischen Menschenrechtskonvention jene Bestimmungen aufhob, die Frauenpaare von der Inanspruchnahme der medizinisch unterstützten Fortpflanzung ausschlossen. In der Schweiz sprechen sich die befragten Reproduktionsmedizinerinnen und -mediziner mehrheitlich für eine Öffnung reproduktionsmedizinischer Verfahren für alleinstehende und homosexuelle Frauen aus. Die Meinung der befragten gesellschaftlichen Akteure ist gespalten. Während sich der befürwortende und der ambivalente Meinungstyp für eine Erweiterung des Zugangs alleinstehender und homosexueller Frauen zur In-vitro-Befruchtung aussprechen, ist der ablehnende Meinungstyp klar dagegen. Die Frage der Zulassung fortpflanzungsmedizinischer Verfahren für homosexuelle Paare steht nicht in Zusammenhang mit Social Freezing. Vielmehr handelt es sich um eine Frage, die unabhängig von der Zulässigkeit und den Grenzen der Eizellenvorsorge zu beantworten sein wird. Aus rechtspolitischen Gründen empfiehlt es sich, die Frage getrennt zu diskutieren.

\section{$9.2 \quad$ Fazit}

Im Fazit werden die zentralen Erkenntnisse der Studie zusammengefasst und anschliessend gestützt darauf Empfehlungen formuliert.

\section{Chancen und Risiken der Technologie noch wenig erforscht}

Während die ovarielle Stimulation und die Befruchtung der Eizellen in vitro seit mittlerweile 40 Jahren praktiziert werden, ist die breite Anwendung der Kryokonservierung von Eizellen eine neuere Erscheinung. Entsprechend ist die Datenlage zu den gesundheitlichen Risiken der Kryokonservierung der Eizellen für die Kinder noch dünn. Besser erforscht sind die mit den beiden anderen Phasen der Technologie verbundenen Risiken. Allerdings werden diese in den Diskussionen zum Social Freezing häufig ausgeblendet. Die ovarielle Stimulation und die Befruchtung der Eizellen in vitro sind mit perinatalen Risiken wie Schwangerschaftsvergiftungen, Frühgeburtlichkeit und niedrigem Geburtsgewicht verbunden. Zudem führen diese reproduktionsmedizinischen Verfahren zu einer leicht erhöhten Fehlbildungsrate. Weiter bestehen Hinweise, dass diese 
Verfahren zu mehr Krebserkrankungen im Kindesalter führen und die kognitive Entwicklung der Kinder beeinträchtigen. Schliesslich begünstigen die reproduktionsmedizinischen Verfahren das Auftreten von Veränderungen am HerzKreislauf-System, die ursächlich mit chronischen Erkrankungen und Todesfällen im Erwachsenenalter in Zusammenhang stehen. Solche Veränderungen werden einerseits auf die erhöhten perinatalen Risiken nach einer In-vitroBefruchtung und andererseits auf durch reproduktionsmedizinische Verfahren ausgelöste epigenetische Veränderungen zurückgeführt. Darunter werden Schaltmechanismen verstanden, welche die Gene ein- oder ausschalten, ohne die Gene selbst zu verändern. Das Ausmass und die Bedeutung dieser Forschungsergebnisse müssen allerdings noch in gross angelegten, gut kontrollierten epidemiologischen Studien geklärt werden. Zu beachten ist zusätzlich, dass die erwähnten Risiken bisher vorwiegend in der medizinisch abgrenzbaren Population unfruchtbarer Paare erforscht wurden, während bei einer Anwendung ohne medizinische Indikation grundsätzlich sehr viel breitere Bevölkerungsschichten einbezogen werden könnten.

Schätzungen zur Wahrscheinlichkeit, ein Neugeborenes mit nach Hause nehmen zu können, beruhen sowohl international als auch in der Schweiz auf unterschiedlichen Grundlagen. Bei Frauen unter 35 Jahren wird die Chance auf ein Kind mit 60 bis 80 Prozent angegeben, wenn 15 bis 20 Eizellen eingefroren worden sind. Sowohl im Ausland als auch in der Schweiz lassen Frauen allerdings aus Kostengründen oder wegen Nebenwirkungen der Behandlung teilweise deutlich weniger Eizellen einfrieren. Da bisher nur wenige Frauen auf ihre eingefrorenen Eizellen zurückgegriffen haben, kann die Erfolgsaussicht dieser Technologie noch nicht zuverlässig beurteilt werden.

\section{Geringe und vorwiegend durch fehlenden Partner motivierte Nachfrage}

Aktuell ist die Nachfrage nach Social Freezing gering. Jährlich lassen geschätzt 300 bis 400 Frauen ihre Eizellen in der Schweiz kryokonservieren. Nur rund die Hälfte der befragten kinderlosen Frauen zwischen 25 und 39 Jahren kennen die Technologie. Die Nachfrage steigt jedoch kontinuierlich. In der Schweiz sind 34 Prozent der befragten kinderlosen Frauen grundsätzlich am Social Freezing interessiert. Sieben Prozent dieser Frauen zeigen ein konkretes Interesse an dem Verfahren. Das Potenzial des Social Freezing dürfte sich somit auf zwi- 
schen 2000 und 10'000 Frauen belaufen, die jährlich ihre Eizellen kryokonservieren lassen.

Deutlich weniger Frauen dürften ihre kryokonservierten Eizellen in vitro befruchten lassen. Gemäss internationalen Studien haben bisher lediglich 10 Prozent der Frauen auf ihre kryokonservierten Eizellen zurückgegriffen. Rund ein Drittel der Frauen mit eingefrorenen Eizellen erachten es als sehr wahrscheinlich, dass sie ihre Eizellen in vitro befruchten lassen werden.

Ausschlaggebende Motivation für die Kryokonservierung der Eizellen ist nicht die Karriere, sondern der fehlende Partner. Gesucht werden Partner, welche dem Bild des «neuen Vaters» entsprechen. Diese Väter nehmen gemeinsam mit den Müttern die Verantwortung für die Kindererziehung wahr und möchten sich intensiv in der Kinderbetreuung engagieren.

Dämpfend auf die Nachfrage wirken sich insbesondere die Kosten aus. Auch die Risiken für das Kind und die Frau reduzieren das Interesse am Social Freezing. Aufgrund der raschen technologischen und sozialen Veränderungen in diesem Bereich sind Nachfragevoraussagen allerdings mit grosser Unsicherheit behaftet.

Grundlagen für einen informierten Entscheid aus ethischer Sicht zentral, jedoch nicht in jedem Fall gegeben

Im Zentrum der individualethischen Diskussion über Social Freezing stehen die Bedeutung eines informierten Entscheides für die Technologie sowie die Berücksichtigung des Kindeswohls. Alle der im Rahmen der Studie interviewten 23 gesellschaftlichen Akteure erachten eine ausführliche Information der Frauen als sehr wichtige Grundlage für einen informierten Entscheid. Eine umfassende Information ist gerade bei Wahleingriffen ohne medizinische Indikation grundsätzlich von grosser Bedeutung. Allerdings besteht bei einer Mehrheit der interviewten gesellschaftlichen Akteure die Befürchtung, dass die Anbieter aufgrund von kommerziellen Interessen die Entscheidung der Frauen beeinflussen. Eine Minderheit der Interviewten schliesst eine solche Beeinflussung durch die Anbieter aus. Die vorliegende Studie liefert Hinweise, dass einzelne Frauen nicht umfassend über die Risiken für Frau und Kind informiert waren. 
Das fortgeschrittene Alter der Mutter im Zentrum der ethischen Diskussion zum Kindeswohl

Es ist auffallend, dass die Risiken der Technologie für das Kindeswohl in der Literatur relativ selten thematisiert werden. Im Fokus der Diskussionen stehen vor allem das Alter der Mutter bei der Befruchtung der Eizellen in vitro und die damit verbundenen medizinischen Risiken für das Kind. Auch die 23 interviewten Schweizer Akteure heben in erster Linie die medizinischen Risiken als Begründung für eine Altersgrenze bei der In-vitro-Befruchtung hervor. Gesellschaftsethische Überlegungen wie die Chancengleichheit der Geschlechter im Zugang zur Reproduktionsmedizin, die Ressourcen älterer Mütter in der Kindererziehung sowie der Einfluss des Social Freezing auf Familienbilder und Generationenbeziehungen spielen eine untergeordnete Rolle. Während eine Mehrheit der interviewten Schweizer Akteure eine fixe Altersgrenze befürwortet, lehnt eine Minderheit diese ab.

Einfluss des Social Freezing auf die gesellschaftlichen Bemühungen zur Vereinbarkeit von Beruf und Familie im Zentrum der Diskussion sozialer Aspekte

Im Fokus der sozialethischen Diskussionen steht der Einfluss des Social Freezing auf die Vereinbarkeit von Beruf und Familie. In der Schweiz überwiegt bei den interviewten gesellschaftlichen Akteuren sowie in den Medien die Skepsis. Es wird befürchtet, dass Social Freezing anstelle von arbeitsrechtlichen, politischen oder gesellschaftlichen Massnahmen zur Vereinbarkeit von Beruf und Familie tritt und sich negativ auf die Chancengleichheit der Geschlechter auswirkt. Mit der ausführlichen Diskussion des Zusammenspiels von Social Freezing und der Vereinbarkeit von Beruf und Familie wird dem Thema allerdings eine Bedeutung beigemessen, die durch die empirischen Befunde nicht gestützt wird. Ausschlaggebend für die Inanspruchnahme des Social Freezing ist primär der fehlende Partner. Vor diesem Hintergrund wäre eine umfassende und systematische Reflexion über das Zusammenspiel von Social Freezing und den Familiengründungsabsichten nicht nur von Frauen, sondern auch von Männern dringlich. Es ist fraglich, inwiefern eine Technologie wie Social Freezing eine Lösung für das soziale Problem eines fehlenden Partners bieten kann. Auch werden andere gesellschaftliche Folgen des Social Freezing wie der Einfluss der Technologie auf Familienbilder und die Generationenbeziehung international und in der Schweiz wenig diskutiert. Die Auswirkungen einer breiten An- 
wendung des Social Freezing auf die öffentliche Gesundheit, insbesondere die Häufigkeit von nicht übertragbaren Krankheiten, sind weder international noch in der Schweiz Gegenstand der Diskussion.

Insgesamt besteht nicht nur im Ausland, sondern auch in den Schweizer Medien sowie bei den interviewten Akteuren ein weites Spektrum an Meinungen zum Social Freezing. Beim Social Freezing handelt es sich um ein sehr persönliches Thema, das stark von individuellen Faktoren wie Alter, Geschlecht und der eigenen Situation bezüglich Kinderwunsch geprägt wird. Während ein Verbot des Social Freezing für die Mehrheit der 23 interviewten Akteure keine Option darstellt, gibt es doch viele Bedenken und Unsicherheiten rund um die Anwendung dieser Technik. Zu verschiedenen Aspekten scheint die Meinungsbildung noch nicht fortgeschritten zu sein.

Weder Arbeitgeber noch soziale Krankenversicherung kommen für die Kostenübernahme infrage

Sowohl international als auch bei den interviewten gesellschaftlichen Akteuren in der Schweiz besteht weitgehend ein Konsens, dass weder der Arbeitgeber noch die soziale Krankenversicherung einen Beitrag an die Kryokonservierung von Eizellen leisten, sondern primär die Frauen dafür aufkommen sollen. Im Zentrum steht das Argument, dass Sozial Freezing aus sozialen und nicht aus medizinischen Gründen eingesetzt wird. Die interviewten Schweizer Akteure lehnen eine Beteiligung der sozialen Krankenversicherung an den Kosten der Befruchtung der aus sozialen Gründen kryokonservierten Eizellen mehrheitlich ab. Nur eine Minderheit könnte sich eine solche Kostenbeteiligung im Sinne der Chancengleichheit zugunsten von weniger gut verdienenden Frauen vorstellen.

Altersgrenze statt Aufbewahrungsfrist im Fokus der rechtlichen Regelung beim Social Freezing

Der Vergleich der gesetzlichen Regelung beim Social Freezing in der Schweiz mit derjenigen in sieben anderen europäischen Ländern zeigt zwei wesentliche Unterschiede. Einerseits sehen die meisten Länder eine Altersgrenze für die Befruchtung der kryokonservierten Eizellen in vitro vor. Andererseits gibt es mit Ausnahme von Belgien kein Land, das eine Aufbewahrungsfrist für die Eizellen 
vorsieht. Im Gegensatz zur Schweiz kann diese Lagerungsdauer in Belgien allerdings im Ermessen der Fertilitätskliniken jederzeit verlängert werden. Während die Aufbewahrungsfrist medizinisch nicht begründet werden kann, sprechen nicht nur medizinische, sondern auch ethische, gesellschaftliche und rechtliche Überlegungen für eine Altersgrenze bei der In-vitro-Befruchtung.

\subsection{Empfehlungen}

Gestützt auf diese Erkenntnisse hat die Projektgruppe zusammen mit der Begleitgruppe sechs Empfehlungen formuliert:

\section{Empfehlung 1: Zurückhaltende Anwendung des Social Freezing}

Social Freezing bedeutet die Anwendung medizinischer Technologien, die für die Behandlung von Unfruchtbarkeit entwickelt wurden, aufgrund einer primär sozialen Indikation. Dies bedingt eine erhöhte ethische Verantwortung im Hinblick auf die eingegangenen medizinischen Risiken sowohl gegenüber den davon betroffenen zukünftigen Kindern als auch in Bezug auf die Folge für die öffentliche Gesundheit. Zu bedenken ist auch, dass einerseits für die Technik der Kryokonservierung von Eizellen erst wenige Daten vorliegen. Andererseits müssen für die schon länger angewendeten reproduktionsmedizinischen Techniken aufgrund neuer Studienergebnisse bedeutsame Auswirkungen auf das Herz-Kreislauf-System betroffener Kinder befürchtet werden. Vor einer breiten Anwendung des Social Freezing sind deshalb verlässlichere Informationen über die medizinischen, sozialen und psychologischen Risiken für die in vitro gezeugten Kinder sowie allfällige Massnahmen zur Reduzierung dieser Risiken notwendig. Bis dahin empfehlen wir daher, die Technologie nur zurückhaltend zu nutzen und anzuwenden.

\section{Empfehlung 2: Förderung der Forschung zu Risiken des Social Freezing}

Um verlässliche Informationen zu medizinischen und anderen Risiken der Fortpflanzungsmedizin zu erhalten, empfehlen wir, die Forschung zum Social Freezing und inbesondere Langzeitstudien mit in vitro gezeugten Kindern zu fördern. 
Als Förderungsgefässe würde einerseits ein Forschungsprogramm des Schweizerischen Nationalfonds (NFP) zur Fortpflanzungsmedizin infrage kommen, in dem neben medizinischen auch ethische, soziale und rechtliche Aspekte des Social Freezing untersucht werden könnten. Andererseits sind internationale Kooperationen zur Erforschung der Langzeitentwicklung der durch Fortpflanzungsmedizin gezeugten Kinder zu fördern, damit genügend grosse Zahlen von Studienteilnehmern erreicht werden, um aussagekräftige Ergebnisse zu den medizinischen Risiken der Reproduktionsmedizin zu generieren.

\section{Empfehlung 3: Die Erfassung der Daten zum Social Freezing gewährleisten}

Im Rahmen dieser Studie konnte lediglich eine grobe Schätzung vorgenommen werden zur Anzahl Frauen, die ihre Eizellen einfrieren lassen. Um die Nachfrage nach dieser Technologie sowie ihre medizinischen, ethischen, gesellschaftlichen und finanziellen Folgen besser einschätzen zu können, sind verlässliche Daten seitens der Kliniken und Labore notwendig. Wir empfehlen, die Datenerfassung zu verbessern. Es sollten Informationen zur Anzahl und zum Alter der Frauen vorliegen, die jährlich ihre Eizellen aus sozialen Gründen kryokonservieren lassen. Weiter sollte erfasst werden, wie viele Frauen und in welchem Alter die eingefrorenen Eizellen in vitro befruchten lassen. Schliesslich sollten Daten zu den Schwangerschafts- und Geburtenraten im Rahmen des Social Freezing gesammelt und ausgewertet werden.

\section{Empfehlung 4: Einen informierten Entscheid sicherstellen}

In Zusammenhang mit Social Freezing wird ein informierter Entscheid einvernehmlich als wichtig erachtet. Die interessierten Frauen sollten umfassende Informationen zum medizinischen Verfahren, zu den Risiken für Frau und Kind, den Erfolgsaussichten, den Kosten und den rechtlichen Rahmenbedingungen erhalten. Es gibt Hinweise, dass interessierte Frauen heute teilweise ungenügend informiert werden. Vor diesem Hintergrund müssen die Informationsanstrengungen verbessert werden. Idealerweise werden Frauen unabhängig und umfassend zu sämtlichen Aspekten des Social Freezing beraten. Prüfenswert wäre, ein Informationsangebot zum Social Freezing bei den kantonalen Fachstellen für Familienplanung und sexuelle Gesundheit anzusiedeln, wie die Beratung bei unerwünschter Schwangerschaft und zu pränataler Diagnostik. 
Die Aufbewahrungsfrist von maximal zehn Jahren sollte zugunsten einer Altersgrenze aufgehoben werden. Gemäss Fortpflanzungsmedizingesetz müssen Eltern ihre in vitro gezeugten Kinder bis zur Volljährigkeit begleiten können. Bei einer durchschnittlichen Lebenserwartung der Frauen von über 80 Jahren könnte mit dieser sozialen Altersgrenze auch eine Befruchtung der kryokonservierten Eizellen bei einer über 60-Jährigen gerechtfertigt werden. Eine Schwangerschaft in fortgeschrittenem Alter bringt jedoch medizinische Risiken mit negativen gesundheitlichen Folgen für Mutter und Kind mit sich. Zudem ist eine Elternschaft in fortgeschrittenem Alter mit sozialen Risiken verbunden. Im Adoptionsrecht wurde entsprechend ein maximaler Altersunterschied zwischen Adoptivkind und Adoptiveltern von 45 Jahren vorgesehen. Um eine Wertungskongruenz zwischen Adoptions- und Fortpflanzungsrecht sicherzustellen, empfehlen wir, eine maximale Altersgrenze von 45 Jahren für Schwangerschaften nach einer In-vitro-Befruchtung vorzusehen. Wie im Adoptionsrecht könnten Ausnahmeregeln vorgesehen werden. In Zusammenhang mit der Einführung einer Altersgrenze müsste die Verwendung der Eizellen neu geregelt werden. Die eingefrorenen Eizellen würden nicht nach Ablauf der Aufbewahrungsfrist wie bei der geltenden Regelung, sondern nach Erreichen der Altersgrenze vernichtet.

Empfehlung 6: Keine Finanzierung des Social Freezing durch die Krankenkassen

Wir sprechen uns gegen die Kostenübernahme für die Kryokonservierung von Eizellen aus sozialen Gründen aus. Gegen eine solche Kostenübernahme spricht, dass diese Technologie aus sozialen Gründen genutzt wird und die Krankenkassen primär für die Kostenfolgen von Krankheiten oder deren Prävention aufkommen. Eine individuelle Vorsorge für den eventuellen Fall, dass bei altersbedingter Unfruchtbarkeit noch ein Kinderwunsch besteht, kann jedoch nicht als Krankheitsprävention bezeichnet werden. 


\section{A1 Anhang: Literaturverzeichnis}

Académie nationale de médecine (2017): La conservation des ovocytes, rapport adopté le 13 juin 2017, Paris.

American Society for Reproductive Medecine (2013): Mature oocyte cryopreservation: a guideline. Fertility and Sterility 2013: 99(1), 37-43.

American Society for Reproductive Medecine (2016): Fertility Drugs and Cancer: a guideline. Fertility and Sterility, 106(7), 1617-26.

Baldwin, K. (2016): Ice, Ice, Baby? A Sociological Exploration of Social Egg Freezing Thesis submitted in partial fulfilment for the award of Doctor of Philosophy. De Montfort University.

Baldwin, K. (2017): I Suppose I Think to Myself, That's the Best Way to Be a Mother: How Ideologies of Parenthood Shape Women's Use of Social Egg Freezing Technology. Sociological Research Online, 22(2), 1-15

Baldwin, K., Culley, L., Hudson, N., Mitchell, H. (2014): Reproductive technology and the life course: Current debates and research in social egg freezing. Human Fertility, 17(3), 170-9.

Barnes, J., Gardiner, J., Melhuish, E., Sutcliffe, A. (2014): The parenting of preschool children by older mothers in the United Kingdom. European Journal of Developmental Psychology, 11 (4), 397-419.

Belaisch-Allart, J., Darai, É., Lévy, G., Epelboin, S., Hédon, B., Merviel, P., Tournaire, M., Ayel, J. P., de Vienne, C. (2013): Réflexions sur les indications sociétales de la préservation de la fertilité féminine. MT Médecine de la Reproduction, Gynécologie Endocrinologie 2013, 15 (1): 111-8.

Bernstein, S., Wiesemann, C. (2014): Should Postponing Motherhood via «Social Freezing» Be Legally Banned? An Ethical Analysis. Laws, 3(2), 282-300.

Blázquez, A., García, D., Rodríguez, A., Vassena, R., Figueras, F., Vernaeve, V. (2016): Is oocyte donation a risk factor for preeclampsia? A systematic review and meta-analysis, J Assist Reprod Genet, 33(7), 85563.

Braun, K., Schultz, S. (2012): Oocytes for research: inspecting the commercialization continuum. New Genetics and Society, 31(2), 135-57. 
Bricknell, R. (2016): When Rumpelstiltskin comes to collect: A labour feminst argument against employersponsored oocyte cryopreservation in Australia. UNSW Law Journal, 39(2), 879-912.

Budds, K., Locke, A., Burr, V. (2016): For some people it isn't a choice, it's just how it happens: Accounts of «delayed» motherhood among middleclass women in the UK. Feminism \& Psychology, 26(2), 170-87.

Camberis, A.-L., McMahon, C. A., Gibson, F. L., Boivin, J. (2016): Maternal Age, Psychological Maturity, Parenting Cognitions, and Mother-Infant Interaction. Infancy, 21(4), 396-422.

Catford, S. R., McLachlan, R. I., O’Bryan, M. K., Halliday, J. L. (2017): Longterm follow-up of intra-cytoplasmic sperm injection-conceived offspring compared with in vitro fertilization-conceived offspring: a systematic review of health outcomes beyond the neonatal period. Andrology, 5(4), 610-21.

Cattapan, A., Hammond, K., Haw, J., Tarasoff, L. A. (2014): Breaking the ice: Young feminist scholars of reproductive politics reflect on egg freezing. International Journal of Feminist Approaches to Bioethics, 7(2), 236-47.

Chen, M., Heilbronn, L. K. (2017): The health outcome of human offspring conceived by assisted reproductive technologies (ART). Journal of Developmental Origins of Health and Disease, 8(4), 388-402.

Cobo, A., Serra, V., Garrido, N., Olmo, I., Pellicer, A., Remohí, J. (2014): Obstetric and perinatal outcome of babies born from vitrified oocytes. Fertility and Sterility, 102(4), 1006-15.

Cutas, D., Smajdor, A. (2015), Postmenopausal Motherhood Reloaded: Advanced Age and In vitro Derived Gametes. Hypatia, 30(2), 386-402.

Daniluk, J. C., Koert, E. (2012): Childless Canadian men's and women's childbearing intentions, attitudes towards and willingness to use assisted human reproduction. Human Reproduction, 27(8), 2405-12.

Daniluk, J. C., Koert, E. (2016): Childless women's beliefs and knowledge about oocyte freezing for social and medical reasons. Human Reproduction, 31(10), 2313-20.

Daniluk, J. C., Koert, E. (2017): Between a rock and a hard place: The reasons why women delay childbearing. International Journal of Healthcare, 3(1), 76-83.

Datta, M. (2017): Egg Freezing on Company Dollars: Making Biological Clock Irrelevant?. DePaul J. Women, Gender and the Law, 6(1), 118-146. 
Davies, M. J., Rumbold, A. R., Marino, J. L., Willson, K., Giles, L. C., Whitrow, M. J., Scheil, W., Moran, L. J., Thompson, J. G., Lane, M., Moore, V. M. (2017): Maternal factors and the risk of birth defects after IVF and ICSI: a whole of population cohort study. An International Journal of Obstetrics and Gynaecology, 124(10), 1537-44.

De Graaf, P. (2016): Non-medical egg freezing sponsored by employers: an ethical evaluation. Thesis Applied Ethics, Utrecht University.

Devine, K., Mumford, S. L., Goldman, K. N., Hodes-Wertz, B., Druckenmiller S., Propst, A. M., Noyes, N. (2015): Baby Budgeting: Oocyte Cryopreservation in Women Delaying Reproduction Can Reduce Cost per Live Birth. Fertility and sterility, 103(6), 1446-53.

De Vore, S., Druckenmiller, S., Grifo, J., Fino, M. E., Goldman, K. N., Noyes, N. (2017): How many does it take? Achievement of euploid blastocyst $(\mathrm{BL})$ as the primary predictor of live birth (LB) in oocyte cryopreservation (OC). Fertility and Sterility, 108(3), e176.

Dondorp, W. J., De Wert, G. M. W. R. (2009): Fertility preservation for healthy women: ethical aspects. Human Reproduction, 24(8), 1779-85.

ESHRE Task Force on Ethics and Law, Dondorp, W., De Wert, G., Pennings, G., Shenfield, F., Devroey, P., Tarlatzis, B., Barri, P., Diedrich, K. (2012): Oocyte cryopreservation for age-related fertility loss. Human Reproduction, 27(5), 1231-7.

ESHRE Working Group on Oocyte Cryopreservation in Europe, Shenfield, F., De Mouzon, J., Scaravelli, G., Kupka, M., Ferraretti, A. P., Prados, F. J., Goossens, V. (2017): Oocyte and ovarian tissue cryopreservation in European countries: statutory background, practice, storage and use. Human Reproduction Open, 2017(1), 1-9.

Fauser, B. C. J. M. et al. (2014): Health outcomes of children born after IVF/ICSI: a review of current expert opinion and literature. Reproductive BioMedicine Online, 28(2), 162-82.

Fleming, T. P., Watkins, A. J., Velazquez, M. A., Mathers, J. C., Prentice, A. M., Stephenson, J., Barker, M., Saffery, R., Chittaranjan, S. Y., Eckert, J., Hanson, M. A., Forrester, T., Gluckman, P. D., Godfrey K. M. (2018): Origins of lifetime health around the time of conception: causes and consequences. Lancet, 391(10132), 1842-52.

Gay, E. T. et al. (2013): Young professional women and elective oocyte cryopreservation: knowledge and desire. Fertility and Sterility, 100(3), 167.

Glujovsky, D., Riestra, B., Sueldo, C., Fiszbajn, G., Repping, S., Nodar, F., Papier, S., Ciapponi, A. (2014): Vitrification versus slow freezing for 
women undergoing oocyte cryopreservation. Cochrane Database of Systematic Reviews, 9.

Goldman, R. H., Racowsky, C., Farland, L. V., Munné, S., Ribustello, L., Fox, J. H. (2017): Predicting the likelihood of live birth for elective oocyte cryopreservation: a counseling tool for physicians and patients. Human Reproduction, 32(4), 853-859.

Goold, I., Savulescu, J. (2009): In favour of freezing eggs for non-medical reasons. Bioethics, 23(1), 47-58.

Gorthi, S., Wright, C. (2010): Is egg freezing for social reasons a good idea? What young women really think. Human Reproduction, 25, 11-14.

Grant, Shelley (2016): Employer sponsored fertility preservation: Debation institutional promotion of reproductive procrastination or choice. Journal of Research in Gender Studies, 6(2), 120-45.

Gunnala, V., Schattman, G. (2017): Oocyte vitrification for elective fertility preservation: the past, present, and future. Current Opinion Obstet Gynecol, 29(1), 59-63.

Guo, X. Y., Liu, X. M., Jin, L., Wang, T. T., Ullah, K., Sheng, J. Z., Huang, H. F. (2017): Cardiovascular and metabolic profiles of offspring conceived by assisted reproductive technologies: a systematic review and metaanalysis. Fertility and Sterility, 107(3), 622-31.

Gupta, J. A. (2006): Towards Transnational Feminisms. European Journal of Women's Studies, 13(1), 23-38.

Hammarberg, K., Kirkman, M., Pritchard, N., Hickey, M., Peate, M., McBain, J., Agresta, F., Bayly, C., Fisher, J. (2007): Reproductive experiences of women who cryopreserved oocytes for non-medical reasons. Human Reproduction, 32(3), 575-81.

Harwood, K. A. (2015): On the ethics of social egg freezing and fertility preservation for nonmedical reasons. Medicolegal and Bioethics, 5, 59-67.

Hens, K. (2017): The Ethics of Postponed Fatherhood. International Journal of Feminist Approaches to Bioethics, 10(1), 103-18.

Hodes-Wertz, B., Druckenmiller, S., Smith, M., Noyes, N. (2013): What do reproductive-age women who undergo oocyte cryopreservation think about the process as a means to preserve fertility? Fertility and Sterility, 100(5), 1343-49.

Jiang, Z., Wang, Y., Lin, J., Xu, J., Ding, G., Huang, H. (2017): Genetic and epigenetic risks of assisted reproduction. Best Practice \& Research Clinical Obstetrics and Gynaecology, 44, 90-104. 
Keurst, A., Boivin, J., Gameiro, S. (2016): Women's intentions to use fertility preservation to prevent age-related fertility decline. Reproductive BioMedicine Online, 32(1), 121-131.

Klüber, C., Krüger, F. P., Wagner, U., Ziller, V. (2016): Wann ist Social-Freezing in Deutschland kosteneffizient? Eine Kosteneffizienzanalyse basierend auf einem Markov Modell. Geburtshilfe Frauenheilkunde 76(10).

Kovács, G. (2013): Frozen future - ethical questions of social egg freezing. Family Forum 3, 201-210.

Kress, H. (2013): Time-lapse-Embryomonitoring und Oozytenvitrifikation: Neue Verfahren in der Reproduktionsmedizin aus ethischer Sicht. Journal für Reproduktionsmedizin und Endokrinologie, 10(3), 163-167.

Lallemant, C., Vassard, D., Nyboe Andersen, A., Schmidt, L., Macklon, N. (2016): Medical and social egg freezing: internet-based survey of knowledge and attitudes among women in Denmark and the UK. Acta Obstet Gynecol Scand, 95(12), 1402-10.

Lazaraviciute, G., Kauser, M., Bhattacharya, S., Haggarty, P., Bhattacharya, S. (2014): A systematic review and meta-analysis of DNA methylation levels and imprinting disorders in children conceived by IVF/ICSI compared with children conceived spontaneously. Human Reproduction Update, 20(6), 840-52.

Levi-Setti, P. E. P., Borini, A., Patrizio, P., Bolli, S., Vigiliano, V., De Luca, R., Scaravelli, G. (2016): ART results with frozen oocytes: data from the Italian ART registry (2005-2013). Journal of Assisted Reproduction and Genetics, 33(1), 123-28.

Lewis, E. I., Missmer, S. A., Farland, L. V., Ginsburg, E. S. (2016): Public support in the United States for elective oocyte cryopreservation. Fertility and Sterility, 106(5), 1183-89.

Lindheim, S. R., Coyne, K., Ayensu-Coker, L., O'Leary, K., Sinn, S., Jaeger, A. S. (2014): The Impact of Assisted Reproduction on Socio-Cultural Values and Social Norms. Advances in Anthropology, 4, 227-42.

Linkeviciute, A., Peccatori, F. A., Sanchini, V., Boniolo, G. (2015): Oocyte cryopreservation beyond cancer: tools for ethical reflection. Journal of Assisted Reproduction and Genetics, 32(8), 1211-20.

Lockwood, G. M. (2011): Social egg freezing: the prospect of reproductive «immortality» or a dangerous delusion? Reproductive BioMedicine Online, 23(3), 334-40.

Marino, J. L., Moore, V. M., Willson, K. J., Rumbold, A., Whitrow, M. J., Giles, L. C., Davies, M. J. (2014): Perinatal Outcomes by Mode of Assisted 
Conception and Sub-Fertility in an Australian Data Linkage Cohort. PLoS ONE, 9(1).

Martin, L. J. (2017): Pushing for the perfect time: Social and biological fertility. Women's Studies International Forum, 62, 91-98.

Masoudian, P., Nasr, A., De Nanassy, J., Fung-Kee-Fung, K., Bainbridge, S. A., El Demellawy, D. (2016): Oocyte donation pregnancies and the risk of preeclampsia or gestational hypertension: a systematic review and metaanalysis. American Journal of Obstetrics \& Gynecology, 214(3), 328-39.

McGinley, A. C. (2016): Subsidized Egg Freezing in Employment: Autonomy, Coercion, or Discrimination? Scholarly Works, 1027.

Mechanick Braverman, A. (2017): Old, older and too old: age limits for medically assisted fatherhood?. Fertility and Sterility, 107(2), 329-33.

Meissner, C., Schippert, C., Von Versen-Höynckcorresponding, F. (2016): Awareness, knowledge, and perceptions of infertility, fertility assessment, and assisted reproductive technologies in the era of oocyte freezing among female and male university students. Journal of Assisted Reproduction and Genetics, 33(6), 719-29.

Meister, T. A., Rimoldi, S. T., Rodrigo, S., Von Arz, R., Messerli, F. H., Sartori, C., Scherrer, U., Rexhay, E. (2018): Association of Assisted Reproductive Technologies with Arterial Hypertension during Adolescence. Journal of the American College of Cardiology, 72(11), 1267-74.

Mertes, H. (2013): The portrayal of healthy women requesting oocyte cryopreservation. Facts, Views \& Vision in Obstetrics and Gynaecology, 5(2), 141-46.

Mertes, H. (2015): «Does Company-Sponsored Egg Freezing Promote or Confine Women's Reproductive Autonomy?» Journal of Assisted Reproduction and Genetics, 32(8), 1205-9.

Mertes, H., Pennings, G. (2011): Social egg freezing: for better, not for worse. Reproductive BioMedicine Online, 23(7), 824-29.

Mertes, H., Pennings, G. (2012): Elective oocyte cryopreservation: who should pay? Human Reproduction, 27(1), 9-13.

Mertes, H., Pennings, G., Wybo, D., de Wert, G. (2012): Implications of oocyte cryostorage for the practice of oocyte donation. Human Reproduction, 27(10), 2886-93.

Milman, L. W., Suneet, S., Sammel, M. D., Cameron, K. D., Gracia, C. (2017): Assessing reproductive choices of women and the likelihood of oocyte 
cryopreservation in the era of elective oocyte freezing. Fertility and Sterility, 107(5), 1214-22.

Myers, C., Daily, Z., Jain, J. (2015): Why Do So Few Women Return to Utilize Cryopreserved Oocytes? Qualitative Insights Into Elective Oocyte Cryopreservation. Fertility and Sterility, 103(2), e30.

Nationale Ethikkommission im Bereich Humanmedizin (2013): Die medizinisch unterstützte Fortpflanzung, Stellungnahme Nr. 22, Bern.

Nationale Ethikkommission im Bereich Humanmedizin (2017): Social Egg Freezing - eine ethische Reflexion, Stellungsnahme Nr. 28, Bern.

Nawroth, F. (2015): Social Freezing Kryokonservierung unbefruchteter Eizellen aus nicht-medizinischen Indikationen. Wiesbaden: Springer Fachmedien.

Pennings, G. (2013): Ethical aspects of social freezing. Gynécologie Obstétrique and Fertilité, 41(9), 521-23.

Perrier, M. (2013): No right time: the significance of reproductive timing for younger and older mothers' moralities. The Sociological Review, 61(1), 69-87.

Peter, C. (2015): Rechtliche, ethische und gleichstellungspolitische Aspekte des Social Freezing. Jusletter 10.

Practice Committee of the American Society for Reproductive Medicine (2016): Electronic address, Fertility drugs and cancer: a guideline. Fertility and Sterility, 106(7), 1617-26.

Pritchard, N., Kirkman, M., Hammarberg, K., McBain, J., Agresta, F., Bayly, C., Hickey, M., Peate, M., Fisher, J. (2017): Characteristics and circumstances of women in Australia who cryopreserved their oocytes for non-medical indications. Journal of Reproductive and Infant Psychology, 35(2), 108-18.

Qin, J. B., Sheng, X. Q., Wang, H. et al. (2017a): Worldwide prevalence of adverse pregnancy outcomes associated with in vitro fertilization/intracytoplasmic sperm injection among multiple births: a systematic review and meta-analysis based on cohort studies. Archives of Gynecology and Obstetrics, 295(3), 577-597.

Qin, J. B., Sheng, X. Q., Wu, D. et al. (2017b): Worldwide prevalence of adverse pregnancy outcomes among singleton pregnancies after in vitro fertilization/intracytoplasmic sperm injection: a systematic review and meta-analysis. Archives of Gynecology and Obstetrics, 295(2), 285301. 
Rimoldi, S. F., Sartori, C., Rexhaj, E., Cerny, D., Von Arx, R., Soria, R., Germond, M., Allemann, Y., Scherrer, U. (2014): Vascular dysfunction in children conceived by assisted reproductive technologies: underlying mechanisms and future implications. Swiss Medical Weekly, 2014, 144.

Robertson, J. A. (2014): Egg freezing and egg banking: empowerment and alienation in assisted reproduction. Journal of Law and the Biosciences, 1(2), 113-36.

Romain, T. (2011): Freezing life, buying time: Consuming cryopreservation services in the United States. Dissertation submitted to the department of anthropology and the committee on graduate studies of Standford University in partial fulfillment of the requirements for the degree of doctor of philosophy.

Roth, S. (2016): Die Fertilitätsreserve aus medizinischer und sozialer Indikation - Eine ethische Analyse. Inaugural-Dissertation zur Erlangung des Doktorgrades der Philosophie (Dr. phil.) durch die Philosophische Fakultät der Heinrich-Heine-Universität Düsseldorf, Düsseldorf.

Rumbold, A. R., Moore, V. M., Whitrow, M. J., Oswald, T. K., Moran, L. J., Fernandez, R. C., Barnhart, K. T., Davies, M. J. (2017): The impact of specific fertility treatments on cognitive development in childhood and adolescence: a systematic review. Human Reproduction, 32(7), 14891507.

Scherrer, U., Rexhaj, E., Allemann, Y., Sartori, C., Rimoldi, S. F. (2015): Cardiovascular dysfunction in children conceived by assisted reproductive technologies. European Heart Journal, 36(25), 1583-89.

Scherrer, U., Rimoldi, S. F., Rexhaj, E., Stuber, T., Duplain, H., Garcin, S., de Marchi, S. F., Nicod, P., Germond, M., Allemann, Y., Sartori, C. (2012): Systemic and Pulmonary Vascular Dysfunction in Children Conceived by Assisted Reproductive Technologies. Circulation, 125(15), 1890-6.

Schick, M., Sexty, R., Ditzen, B., Wischmann, T. (2017): Einstellungen zum Einfrieren von Eizellen aus nicht medizinischen Gründen unter soziokultureller Perspektive. Geburtshilfe und Frauenheilkunde, 77(07): 747755.

Schuman, L. et al. (2013): Women pursuing non-medical oocyte cyropreservation would consider non-genetic methods of family building such as adoption or ovum donation. Fertility and Sterility, 100(3), 66. 
Seggers, J., Pontesilli, M., Ravelli, A. C. J., Painter, R. C., Hadders-Algra, M., Heineman, M. J., Repping, S., Mol, B. W. J., Roseboom, T. J., Ensing, S. (2016): Effects of in vitro fertilization and maternal characteristics on perinatal outcomes: a population-based study using siblings. Fertility and Sterility, 105(3), 590-598.

Seyler, H. (2014): Kryokonservierung von Eizellen aus sozialen Gründen «Social Freezing». Pro familia medizin, 3, 2-9.

Shkedi-Rafid, S., Hashiloni-Dolev, Y. (2012): Egg freezing for non-medical uses: the lack of a relational approach to autonomy in thenew Israeli policy and in academic discussion. Journal of Medical Ethics, 38(3), 154-157.

Simoni, H. (2012): Sozialwissenschaftliche Grundlagen zu den Konzepten «Kindeswohl, Familie und Elternschaft» im Fortpflanzungsmedizingesetz, Studien im Auftrag des Bundesamtes für Gesundheit, Marie Meierhofer Institut für das Kind.

Sobotka, T. (2009): Shifting Parenthood to Advanced Reproductive Ages: Trends, Causes and Consequences: Intergenerational Justice Review, 9(2), 56-61.

Spangmose, A. L., Malchau, S. S., Schmidt, L., Vassard, D., Rasmussen, S., Loft, A., Forman, J., Pinborg, A. (2017): Academic performance in adolescents born after ART - a nationwide registry-based cohort study. Human Reproduction, 32(2), 447-56.

Stanton, C. et al. (2014): A Survey on Awareness and Interest Towards Proactive Egg Freezing among Women 25-35 Years Old. Fertility and Sterility, 101(2), e34-e35.

Stoop, D., Maes, E., Polyzos, N. P., Verheyen, G., Tournaye, H., Nekkebroeck, J. (2015): Does oocyte banking for anticipated gamete exhaustion influence future relational and reproductive choices? A follow-up of bankers and non-bankers. Human Reproduction, 30(2), 338-44.

Stoop, D., Nekkebroeck, J., Devroey, P. (2011): A survey on the intentions and attitudes towards oocyte cryopreservation for non-medical reasons among women of reproductive age. Human Reproduction, 26(3), 655-61.

Vallejo, V., Lee, J. A., Schuman, L., Witkin, G., Cervantes, E., Sandler, B., Copperman, A. B. (2013): Social and psychological assessment of women undergoing elective oocyte cryopreservation: 7-year analysis. Open Journal of Obstetrics and Gynecology, 17(3), 1-7. 
Van der Ven, K., Pohlmann, M., Hössle, C., Pohlmann, M., Hößle, C. (2017): Social Freezing. Die Möglichkeiten der modernen Fortpflanzungsmedizin und die ethische Kontroverse. Wiesbaden: Springer Fachmedien.

Van Loendersloot, L. L., Moolenaar, L. M., Mol, B. W. J., Repping, S., van der Veen, F., Goddijn, M. (2011): Expanding reproductive lifespan: a costeffectiveness study on oocyte freezing. Human Reproduction, 26(11), 3054-60.

Vermeiden, J. P. W., Bernardus, R. E. (2013): Are imprinting disorders more prevalent after human in vitro fertilization or intracytoplasmic sperm injection?. Fertility and Sterility, 99(3), 642-51.

Von Wolff, M. (2013): Anlage einer Fertilitätsreserve bei nicht-medizinischen Indikationen «Social freezing»: Sinn oder Unsinn?. Schweizerische Ärztezeitung, 94, 10.

Von Wolff, M., Germeyer, A., Nawroth, F. (2015): Anlage einer Fertilitätsreserve bei nichtmedizinischen Indikationen. Deutsches Ärzteblatt, 2015(3), 27-32.

Von Wolff, M., Stute, P. (2015): Cryopreservation and transplantation of ovarian tissue exclusively to postpone menopause: technically possible but endocrinologically doubtful. Reproductive BioMedicine Online, 31(6), 718-21.

Waldby, C. (2008): Oocyte markets: women's reproductive work in embryonic stem cell research. New Genetics and Society, 27(1), 19-31.

Waldby, C. (2014): «Banking time»: egg freezing and the negotiation of future fertility. Culture, Health \& Sexuality, an International Journal for Research, Intervention and Care, 17(4), 470-482.

Weinrauch, L. A., Gerhard-Herman, M. D., Mendelson, M. M. (2018): Epigenetics. Is the Mode of Conception a Marker for Future Cardiovascular Risk?. Journal of the American College of Cardiology, 72(11), 1275-77.

Wennberg, A.-L., Rodriguez-Wallberg, K. A., Milsom, I., Brännström, M. (2016): Attitudes towards new assisted reproductive technologies in Sweden: a survey in women 30-39 years of age. Acta Obstetricia et Gynecologica Scandinavica, 95(1), 38-44.

Witkin, G. et al. (2013): What makes a woman freeze: the impetus behind patients' desires to undergo elective oocyte cryopreservation. Fertility and Sterility, 100(3), 24. 
Woodward, J. T., MacDuffie, K. E. (2016): Individual and familily outcomes of first-time parents older than 40 years: implications of later-life parenting, in Stevenson Eleanor L., Hershberger Patricia E. (Ed.), Fertility and assisted reproductie technology (ART). Theory, Research, Policy, and Practice for Health Care Practioners. New York: Springer Publishing.

Wunder, D. (2013): Social Freezing in Switzerland and worldwide - a blessing for women today? Swiss Medical Weekly, 143.

Wunder, D. (2014): Gutachten «Höchstalter der Frau für Fortpflanzungsmedizin» im Auftrag des Bundesamtes für Gesundheit, Bern. 


\section{A2 Anhang: Medienartikel}

Neue Zürcher Zeitung NZZ/NZZ am Sonntag

Althaus, Nicole (2014a): Eiszeit für die Emanzipation, in: NZZ am Sonntag, 18.5.2014, Nr. 20, S. 20.

Althaus, Nicole (2014b): Bei Apple dürfen Sie Ihren Babywunsch auf Eis legen, in: NZZ am Sonntag, 19.10.2014, Nr. 42, S. 20.

Bracher, Katharina (2014): Für das späte Kind nach Spanien, in: NZZ am Sonntag, 22.6.2014, Nr. 25, S. 12.

Klott, Anna (2013): Wenn Frauen ihren Kinderwunsch auf Eis legen, in: NZZ am Sonntag, 28.4.2013, Nr. 17, S. 55.

Schipper, Ori (2016): Künstlich gezeugte Kinder scheinen eine Veranlagung zu Herz-Kreislauf-Problemen zu haben, in: NZZ am Sonntag, 10.7.2016, Nr. 28, S. 49.

Schweri, Thomas (2014): Alte junge Mütter, in: Neue Zürcher Zeitung, Briefe an die NZZ, 6.1.2014, S. 14.

Streeck, Nina (2015a): Fortpflanzung ohne Sex wird bald zur Normalität - Das verändert die Art und Weise, wie wir lieben, arbeiten und Familie gründen, in: NZZ am Sonntag, 25.1.2015, Nr. 4, S. 66.

Streeck, Nina (2015b): «Biologische Lösungen sind der falsche Weg», in: NZZ am Sonntag, 25.1.2015, Nr. 4, S. 66.

Neue Luzerner Zeitung NLZ

Ackermann, Jürg (2013): Spätes Mutterglück mit gefrorenem Ei, in: Neue Luzerner Zeitung, 3.12.2013, S. 3.

Ackermann, Jürg (2014): Altersgrenze für Schwangerschaften, in: Neue Luzerner Zeitung, 10.11.2014, S. 4.

Neue Nidwaldner Zeitung

Wittenwiler, Mario (2013): Diese Jus-Studentin holt die Note 6,0, in: Neue Nidwaldner Zeitung, 16.3.2013, S. 30. 


\section{St. Galler Tagblatt}

Ackermann, Jürg (2014): Keine Eizellen für ältere Mütter, in: St. Galler Tagblatt, 8.11.2014, S. 5.

Fischer De Santi, Katja (2014): Die Zeit einfrieren, in: St. Galler Tagblatt, 25.10.2014, S. 31.

\section{Tages-Anzeiger}

Meili, Matthias (2011): Analyse - Glückliche Karrierekinder, in: Tages-Anzeiger, 10.12.2011, S. 13.

Meili, Matthias (2016): Wunschkind, aber später, bitte!, in: Tages-Anzeiger, 27.5.2016, S. 46.

Raus, Simone (2014): Den Kinderwunsch auf Eis gelegt, in: Tages-Anzeiger, 17.10.2014, S. 9.

Straumann, Felix (2011): Das Geschäft mit der Torschlusspanik, in: TagesAnzeiger, 9.12.2011, S. 44.

Wirthlin, Annette (2016): Trügerische Hoffnung auf spätes Mutterglück, in: Tages-Anzeiger, Mamablog, 2.4.2016, S. 2.

\section{SonntagsZeitung}

Marinka, Claudia (2014): Das Ei aus der Kühlbox, in: SonntagsZeitung, 11.5.2014, S. 49.

\section{Minuten}

Dubler, Fabrice (2017): Eizellen einfrieren: Nachfrage steigt bei Schweizerinnen, in: 20 Minuten, 2.3.2017.

\section{Berner Zeitung}

Walser, Brigitte (2015): Junge sind offener, in: Berner Zeitung, 29.10.2015, S. 16. 
Le Matin

Petit-Pierre, Marie-Christine (2014): La fécondation in vitro recule légèrement en Suisse, in: Le Matin, 27.4.2014, S. 74.

Le Temps

Petit-Pierre, Marie-Christine (2012): Peut-on suspendre le temps en congelant ses ovocytes?, in: Le Temps, 24.11.2012.

Weltwoche

Stämpfli, Regula (2014): Geschichten - Kalte Perfektion, in: Weltwoche, 6.11.2014, Nr. 45, S. 34-35.

Mittelland Zeitung

Schuppisser, Raffael (2013): Eizellen lassen sich einfrieren und für den späteren Gebrauch aufheben - so kann man auch noch mit 47 schwanger werden wie Halle Berry, in: Mittelland Zeitung, 13.10.2013.

Die Nordwestschweiz

Schuppisser, Raffael (2014): Jetzt kommen die iBabys, in: Die Nordwestschweiz, 16.10.2014.

Basler Zeitung

Bahnerth, Michael (2014): Tiefgekühlter Kinderwunsch - Fünf Meinungen, in: Basler Zeitung, 4.11.2014, S. 2. 
Merli, Nina (2014): Regula Stämpfli (52) zum Baby nach Businessplan: «Dieser Vorschlag ist sexistisch», in: Blick, 17.10.2014, S. 8.

Schirm-Gasser, Carmen (2014): Das Baby aus der Tiefkühltruhe - Einfrieren von Eizellen boomt, in: SonntagsBlick, 2.11.2014, S. 8.

SonntagsBlick (2014): Eizellen für später einfrieren? - So denkt die Schweiz, in: SonntagsBlick, 14.12.2014, S. 8.

\section{Zürichsee-Zeitung}

Sattler, Seraina (2017): Nicht alles, was machbar ist, ist auch richtig, in: Zürichsee-Zeitung, 6.5.2017.

$S D A$

Schweizerische Depeschenagentur (2015): Fortpflanzungsmedizin überfordert eine Mehrheit der Schweizer, 28.10.2015.

Diverse (Fach-)Zeitschriften/Onlinemedien

Achermann, Barbara (2015): Wunschkind und künstliche Befruchtung - Interview mit Barbara Bleisch, in: Annabelle, Nr. 10/2015.

Brauer, Susanne (2015): «Mein Bauch gehört mir!» Alte Freiheit in Zeiten neuer Reproduktionstechniken, in: FAMA, Nr. 2/2015.

C., Sandra (Datum unbekannt): Das Problem liegt im Kopf, nicht im Unterleib, in: Schweizer Illustrierte.

Faschon, Christiane (2014): Erst Karriere - später Kinder?, in: Pfarreiblatt der Bistumskantone Schaffhausen und Thurgau, Nr. 22/2014.

Läubli, Martina (2014): Gezeugt oder gemacht? - Umstrittene Fortpflanzungsmedizin, in: Aufbruch, 14.11.2014.

Peter, Christian (2015): Rechtliche, ethische und gleichstellungspolitische Aspekte des Social Freezing, in: Jusletter, 10.8.2015. 
Swissmom (2015): Familienplanung auf Eis, $<$ https://www.swissmom.ch/aktuell/wissen/2015/familienplanung-aufeis/>, Zugriff: 9.11.2017.

Urech-Ruh, Cornelia; Schneider, Mischa; Hohl, Michael K. (2017): Social freezing: Weg vom negativen Touch!, in: Frauenheilkunde aktuell, Nr. 1, S. 1-9.

Vakaridis, Mary (2015): Ovules congelés: un créneau porteur, in: Bilan, 15.4.2015.

Wirthlin, Annette (Datum unbekannt): Man kann die Zeit nicht zurückdrehen. $<$ http://www.wireltern.ch/artikel/0216-man-kann-die-zeit-nichtzurueckdrehen>, Zugriff 8.11.2017.

Wohlgemuth, Julia (2014): Eizellen einfrieren - Wenn der Kinderwunsch aufgeschoben wird, $<$ https://www.familienleben.ch/schwangerschaft/kinderwunsch/eizellen -einfrieren-mit-kryotransfer-zum-baby-3648>, Zugriff: 9.11.2017. 


\section{A3 Anhang: Q-Methode}

\section{Literaturverzeichnis}

Brown, Steven R. (1980): Political Subjectivity: Applications of Q Methodology in Political Science. New Haven and London: Yale University Press.

Coogan, Joy; Herrington, Neil (2011): Q methodology: an overview. In: Research in Secondary Teacher Education, 1(2), 24-28.

Müller, Florian H.; Kals, Elisabeth (2004): Die Q-Methode. Ein innovatives Verfahren zur Erhebung subjektiver Einstellungen und Meinungen. In: Forum Qualitative Sozialforschung, 5(2), Art. 34, <http://www.qualitativeresearch.net/index.php/fqs/article/view/600/1301> (Zugriff: 03/2018).

Schmolck, Peter (2002). QMethod page (Universität der Bundeswehr München): <http://www.rz.unibw-muenchen.de/ p41bsmk/qmethod/> (Zugriff: 03/2018).

Schmolck, Peter (2014): PQMethod Manual, Version März 2014. http:// schmolck.userweb.mwn.de/qmethod/pqmanual.htm (Zugriff: 03/2018).

Shinebourne, Pnina (2009): Using Q-Method in Qualitative Research. IIQM International Journal of Qualitative Methods, 8(1), 97.

Watts, Simone; Stenner, Paul (2005): Doing Q methodology: theory, method and interpretation. In: Qualitative Research in Psychology, 2, 67-91.

\section{Gesprächspartnerinnen und -partner}

Die nachfolgende Tabelle listet die Gesprächspartnerinnen und -partner, Vertretende von politischen Parteien und gesellschaftlichen Gruppierungen, auf, die bei der Anwendung der Q-Methode interviewt wurden.

\begin{tabular}{|l|l|}
\hline Dorothea Forster & Evangelische Frauen Schweiz (Co-Präsidentin) \\
\hline Regula Ott & $\begin{array}{l}\text { Schweizerischer Katholischer Frauenbund, Leitung Bildung, } \\
\text { Ethik und Theologie }\end{array}$ \\
\hline $\begin{array}{l}\text { François-Xavier } \\
\text { Putallaz }\end{array}$ & $\begin{array}{l}\text { Präsident, Kommission für Bioethik der Schweizer Bischofs- } \\
\text { konferenz }\end{array}$ \\
\hline Pascale Steck & Biorespect (Geschäftsführerin) \\
\hline Jérôme Cosandey & Avenir Suisse (Forschungsleiter Finanzierbarere Sozialpolitik) \\
\hline Regula Bühlmann & Schweizerischer Gewerkschaftsbund (Politische Sekretärin) \\
\hline
\end{tabular}




\begin{tabular}{|l|l|}
\hline Pius Zängerle & Curafutura (Direktor) \\
\hline Kathrin Bertschy & $\begin{array}{l}\text { Alliance F (Co-Präsidentin) } \\
\text { Grünliberale (Nationalrätin) }\end{array}$ \\
\hline $\begin{array}{l}\text { Vivian Fankhauser- } \\
\text { Feitknecht }\end{array}$ & $\begin{array}{l}\text { Eidgenössische Kommission für Frauenfragen/ } \\
\text { Alliance F }\end{array}$ \\
\hline Markus Gygli & Männer.ch (Präsident) \\
\hline Erika Ziltener & Patientenstelle Zürich (Geschäftsleiterin) \\
\hline Dr. med. Yvonne Gilli & $\begin{array}{l}\text { Sexuelle Gesundheit Schweiz (Präsidentin) } \\
\text { Grüne (ehem. Nationalrätin) }\end{array}$ \\
\hline Conrad Engler & Verein Kinderwunsch \\
\hline Muriel Degen Koch & Marie Meierhofer Institut für das Kind \\
\hline Barbara Stocker-Kalberer & Schweizer Hebammenverband \\
\hline Tamara Papst & Insieme (Vorstand) \\
\hline Seraina Grünig & $\begin{array}{l}\text { Schweizerische Konferenz der kantonalen Gesundheitsdirekto- } \\
\text { reninnen und -direktoren GDK (Projektleiterin Medizin und Qualität) }\end{array}$ \\
\hline Isabelle Moret & FDP (Nationalrätin) \\
\hline Rosmarie Quadranti & BDP (Nationalrätin) \\
\hline Marianne Streiff-Feller & EVP (Nationalrätin) \\
\hline Verena Herzog & SVP (Nationalrätin) \\
\hline Yvonne Schärli-Gerig & $\begin{array}{l}\text { SP (ehem. Regierungsrätin Kanton Luzern) } \\
\text { Präsidentin eidgenössische Kommission für Frauenfragen }\end{array}$ \\
\hline Karin Stadelmann & CVP Kanton Luzern (Co-Präsidentin) \\
\hline
\end{tabular}


Tabelle 6:

Statements zu Social Freezing mit Faktorenwerten (Q-sort values) für die Faktoren

\begin{tabular}{|c|c|c|c|c|}
\hline \multirow[t]{2}{*}{$\mathrm{Nr}$. } & \multirow[t]{2}{*}{ Statement } & \multicolumn{3}{|c|}{ Faktoren } \\
\hline & & A & B & C \\
\hline \multicolumn{5}{|c|}{ Ethische Dimension } \\
\hline E1 & $\begin{array}{l}\text { Überzählige Eizellen können problemlos vernichtet werden, da Ei- } \\
\text { zellen weder ethisch noch rechtlich ein schützenswürdiges Gut } \\
\text { darstellen. }\end{array}$ & -1 & -1 & 0 \\
\hline E2 & $\begin{array}{l}\text { Überzählige Eizellen sollten an unfruchtbare Frauen gespendet } \\
\text { werden dürfen, die sich im Gegenzug an den Kosten beteiligen. } \\
\text { So könnten sich auch Frauen mit eingeschränkten Mitteln diese } \\
\text { Technik leisten. }\end{array}$ & $-4^{*}$ & 2 & 2 \\
\hline E3 & $\begin{array}{l}\text { Überzählige Eizellen sollten nicht gegen eine Kostenbeteiligung } \\
\text { gespendet werden dürfen, da dies zu einer ethisch bedenklichen } \\
\text { Kommerzialisierung der Eizellen führt. }\end{array}$ & $4^{*}$ & 0 & -1 \\
\hline E4 & $\begin{array}{l}\text { Eine Spende von überzähligen Eizellen zu einem späteren Zeit- } \\
\text { punkt, wenn die Spenderin die Eizellen sicher nicht mehr braucht, } \\
\text { ist aus ethischer Perspektive weniger heikel als eine Eizellspende } \\
\text { unmittelbar nach der Entnahme. }\end{array}$ & -2 & 0 & -1 \\
\hline E5 & $\begin{array}{l}\text { Eine Spende überzähliger Eizellen an die Forschung ist ethisch } \\
\text { weniger heikel als eine Spende an eine unfruchtbare Frau. Die } \\
\text { Spenderinnen sind so nicht mit der Vorstellung konfrontiert, dass } \\
\text { irgendwo ein Kind von ihnen aufwächst. }\end{array}$ & -2 & -3 & $1^{*}$ \\
\hline E6 & $\begin{array}{l}\text { Social Freezing ist eine sinnvolle Alternative zur Eizellspende. } \\
\text { Denn beim Social Freezing unterzieht sich die Frau selbst dem } \\
\text { aufwendigen Verfahren der Eizellentnahme - und nicht eine } \\
\text { fremde und unbeteiligte Eizellspenderin. }\end{array}$ & 0 & 0 & $4^{*}$ \\
\hline E7 & $\begin{array}{l}\text { Social Freezing ist eine sinnvolle Alternative zur Eizellspende, } \\
\text { weil eine genetische Verbindung zwischen Mutter und Kind be- } \\
\text { stehen bleibt. }\end{array}$ & 0 & 2 & $5^{*}$ \\
\hline$E 8^{* *}$ & $\begin{array}{l}\text { Nur wenn Frauen alle Pro und Kontras des Social Freezing ken- } \\
\text { nen, können sie ihre informierte Einwilligung erteilen. Ein infor- } \\
\text { mierter Entscheid ist eine wichtige Voraussetzung für die Aus- } \\
\text { übung der Fortpflanzungsfreiheit. }\end{array}$ & 5 & 4 & 4 \\
\hline
\end{tabular}




\begin{tabular}{|c|c|c|c|c|}
\hline $\mathrm{Nr}$. & Statement & Fakt & en & C \\
\hline E9 & $\begin{array}{l}\text { Eizellen sollten ohne medizinischen Grund nur dann eingefroren } \\
\text { werden dürfen, wenn die Information und die Werbung durch die } \\
\text { Reproduktionskliniken nicht irreführend sind. }\end{array}$ & 3 & 0 & 2 \\
\hline E10 & $\begin{array}{l}\text { Eine Garantie für ein Baby gibt es mit Social Freezing nicht. } \\
\text { Durch das Angebot von Reproduktionskliniken könnten sich junge } \\
\text { Frauen in falscher Sicherheit wiegen. }\end{array}$ & 3 & 3 & 2 \\
\hline E11 & $\begin{array}{l}\text { Die Inanspruchnahme des Social Freezing ist nicht Ausdruck } \\
\text { eines informierten und autonomen Entscheides der Frau, sondern } \\
\text { Ausdruck gesellschaftlicher Zwänge. }\end{array}$ & 1 & $2^{*}$ & $-5^{*}$ \\
\hline E12 & $\begin{array}{l}\text { Es gibt keine guten Gründe, einer gut informierten Frau die Mög- } \\
\text { lichkeit des Social Freezing zu verwehren, nur weil keine medizi- } \\
\text { nische Notwendigkeit (wie z.B. Krebs) vorliegt. }\end{array}$ & $-2^{*}$ & 3 & 3 \\
\hline E13 & $\begin{array}{l}\text { Keine Frau trifft eine Entscheidung zum Social Freezing leichtfer- } \\
\text { tig. Dafür ist es ein zu unangenehmer Prozess, der keine hun- } \\
\text { dertprozentige Garantie für Erfolg verspricht. }\end{array}$ & 2 & 3 & $5^{*}$ \\
\hline E14 & $\begin{array}{l}\text { Es bestehen nur wenige Studien zu den gesundheitlichen Folgen } \\
\text { der Eizellkonservierung für das Kind. Deshalb sollte die Technik } \\
\text { nicht breit angewendet werden dürfen. }\end{array}$ & $4^{*}$ & -2 & -1 \\
\hline E15 & $\begin{array}{l}\text { Bei Techniken wie Social Freezing besteht die Gefahr, dass das } \\
\text { Kindeswohl gegenüber elterlichen Wünschen in den Hintergrund } \\
\text { tritt. }\end{array}$ & $5^{*}$ & $-1^{*}$ & $-4^{*}$ \\
\hline E16 & $\begin{array}{l}\text { Für die künstliche Befruchtung der Eizellen sollte eine Altersgren- } \\
\text { ze definiert werden, weil eine Schwangerschaft in fortgeschritte- } \\
\text { nem Alter mit grösseren Risiken für Mutter und Kind verbunden } \\
\text { ist. }\end{array}$ & $4^{*}$ & $-2^{*}$ & $2^{*}$ \\
\hline E17 & $\begin{array}{l}\text { Die Einführung einer Altersgrenze für die künstliche Befruchtung } \\
\text { ist willkürlich, weil die Risiken einer Schwangerschaft in fortge- } \\
\text { schrittenem Alter von Frau zu Frau sehr unterschiedlich sind. } \\
\text { Ärztinnen und Ärzte sollen das Risiko beurteilen. }\end{array}$ & $-3^{*}$ & 1 & 0 \\
\hline E18 & $\begin{array}{l}\text { Eine Altersgrenze für die künstliche Befruchtung von Eizellen ist } \\
\text { sinnvoll. Denn ältere Mütter haben zu wenig Energie, um sich } \\
\text { angemessen um ein Kleinkind oder pubertierende Teenager zu } \\
\text { kümmern. }\end{array}$ & $1^{*}$ & $-4^{*}$ & $-1^{*}$ \\
\hline
\end{tabular}




\begin{tabular}{|c|c|c|c|c|}
\hline \multirow[t]{2}{*}{ Nr. } & \multirow[t]{2}{*}{ Statement } & \multicolumn{3}{|c|}{ Faktoren } \\
\hline & & A & B & C \\
\hline E19 & $\begin{array}{l}\text { Eine Altersgrenze für die künstliche Befruchtung der Eizellen ist } \\
\text { nicht notwendig, da Kinder von älteren Müttern von stabileren } \\
\text { Familienverhältnissen und einer grösseren finanziellen Sicherheit } \\
\text { profitieren. }\end{array}$ & -4 & $1^{*}$ & -3 \\
\hline E20 & $\begin{array}{l}\text { Eine Altersgrenze für die künstliche Befruchtung der Eizellen ist } \\
\text { notwendig, um zu verhindern, dass ein Kind früh seine Mutter ver- } \\
\text { liert. }\end{array}$ & 0 & -3 & -3 \\
\hline E21 & $\begin{array}{l}\text { Dank der höheren Lebenserwartung kann eine Frau heute pro- } \\
\text { blemlos später Mutter werden und ihr Kind bis zur Volljährigkeit } \\
\text { begleiten. }\end{array}$ & $-2^{*}$ & $1^{*}$ & $3^{*}$ \\
\hline E22** & $\begin{array}{l}\text { Auch bei älteren Männern steigen die gesundheitlichen Risiken } \\
\text { für das Kind. Wenn man eine Altersgrenze für die künstliche Be- } \\
\text { fruchtung einführt, sollte diese deshalb für Frauen und Männer } \\
\text { gleichermassen gelten. }\end{array}$ & -1 & 0 & 1 \\
\hline$E 23^{* *}$ & $\begin{array}{l}\text { Ältere Väter sind gesellschaftlich besser akzeptiert als ältere } \\
\text { Mütter. Wenn die Chancengleichheit der Geschlechter gefördert } \\
\text { werden soll, müsste für die künstliche Befruchtung die gleiche } \\
\text { Altersgrenze für Männer und Frauen gelten. }\end{array}$ & -1 & 0 & 0 \\
\hline E24 & $\begin{array}{l}\text { Eine Frau, die Social Freezing nutzt, praktiziert eine verantwor- } \\
\text { tungsbewusste Familienplanung. Sie tritt aus der Opfer- und } \\
\text { Verzichtsrolle heraus und nimmt ihr Leben selbst in die Hand. }\end{array}$ & $-3^{*}$ & $-5^{\star}$ & $1^{*}$ \\
\hline E25 & $\begin{array}{l}\text { Wenn eine Frau zu einem gewissen Zeitpunkt keine Kinder mehr } \\
\text { bekommen kann, hat das seine Gründe. Man sollte sich der Natur } \\
\text { nicht immer so widersetzen. }\end{array}$ & $3^{*}$ & -5 & -3 \\
\hline \multicolumn{5}{|c|}{ Gesellschaftliche Dimension } \\
\hline G26 & $\begin{array}{l}\text { Social Freezing ist ein Schritt zur Befreiung der Frau von ihren } \\
\text { «natürlichen Fesseln». Die Technik leistet einen Beitrag zur Un- } \\
\text { abhängigkeit der Frau von ihrer aktuellen beruflichen oder part- } \\
\text { nerschaftlichen Situation. }\end{array}$ & -4 & -4 & $-1^{*}$ \\
\hline G27 & $\begin{array}{l}\text { Gerade zum Karriereanfang sind Frauen um die } 30 \text { dem Druck } \\
\text { ausgesetzt, sich für Kinder oder Karriere zu entscheiden. Mit So- } \\
\text { cial Freezing kann diese Entscheidung bis zu zehn Jahre verzö- } \\
\text { gert werden. Die Frauen werden so ein Stück weit vom Druck } \\
\text { erlöst. }\end{array}$ & 0 & $-4^{*}$ & 0 \\
\hline
\end{tabular}




\begin{tabular}{|c|c|c|c|c|}
\hline $\mathrm{Nr}$. & Statement & Fakt & en & C \\
\hline G28 & $\begin{array}{l}\text { Unternehmen könnten Schwangerschaften zukünftig als «ver- } \\
\text { meidbar» ansehen. Die Anwendung des Social Freezing würde so } \\
\text { zu einer sozialen Vorgabe für Frauen, wenn sie sich noch be- } \\
\text { ruflich entwickeln wollen. }\end{array}$ & 2 & 4 & $-2^{*}$ \\
\hline G29 & $\begin{array}{l}\text { Wenn sich Social Freezing in der Gesellschaft etabliert, könnte } \\
\text { die Bereitschaft von Unternehmen abnehmen, die Vereinbarkeit } \\
\text { von Beruf und Familie (z.B. durch flexiblere Arbeitszeiten oder } \\
\text { Subventionierung von Kinderbetreuung) zu unterstützen. }\end{array}$ & $2^{*}$ & $5^{*}$ & $-2^{*}$ \\
\hline G30 & $\begin{array}{l}\text { Statt ein Umfeld zu schaffen, das partnerschaftliche Kinderbe- } \\
\text { treuung und berufliche Karriere beider Partner ermöglicht, wird die } \\
\text { Verantwortung mit Social Freezing einmal mehr an die Frau } \\
\text { abgeschoben. Das verstärkt die Diskriminierung von Frauen. }\end{array}$ & 3 & 5 & $-3^{*}$ \\
\hline G31 & $\begin{array}{l}\text { Social Freezing kann zwar Massnahmen zur Förderung von Beruf } \\
\text { und Familie auf gesellschaftlicher Ebene (wie z.B. subventionierte } \\
\text { Kinderbetreuung) nicht ersetzen. Aber die Technologie verhindert } \\
\text { diese auch nicht. }\end{array}$ & 0 & 1 & 2 \\
\hline G32 & $\begin{array}{l}\text { Die Anwendung von medizinischen Lösungen der Fortpflanzungs- } \\
\text { medizin für soziale oder gesellschaftliche Probleme (wie fehlender } \\
\text { Partner, Unvereinbarkeit von Karriere und Kindern) sollte unter- } \\
\text { bunden werden. }\end{array}$ & 1 & 1 & $-5^{*}$ \\
\hline G33 & $\begin{array}{l}\text { Gute Medizin umfasst nicht nur die Behandlung, sondern auch die } \\
\text { Prävention von Krankheiten. Social Freezing ist eine präventive } \\
\text { Massnahme einer ungewollten Unfruchtbarkeit in fortgeschritte- } \\
\text { nem Alter. }\end{array}$ & -3 & -1 & 0 \\
\hline G34 & $\begin{array}{l}\text { Social Freezing ermöglicht eine Mutterschaft jenseits der biologi- } \\
\text { schen Altersgrenze und verändert so langfristig die Mutterrolle. Es } \\
\text { ist bedenklich, wenn die Mutter einer 20-jährigen Tochter schon } \\
\text { im Altersheim lebt. }\end{array}$ & $2^{*}$ & $-2^{*}$ & $-4^{*}$ \\
\hline G35** & $\begin{array}{l}\text { Social Freezing führt zu grösseren Altersunterschieden innerhalb } \\
\text { einer Familie. Dies erschwert die Pflegearbeit zwischen den Ge- } \\
\text { nerationen, weil die Grosseltern die Mütter nicht mehr bei der } \\
\text { Kindererziehung entlasten können. }\end{array}$ & -1 & -1 & -2 \\
\hline G36** & $\begin{array}{l}\text { Social Freezing führt dazu, dass die älteren Mütter doppelt belas- } \\
\text { tet werden, weil sie sich neben den Kindern auch noch um ihre } \\
\text { betagten Eltern kümmern müssen. }\end{array}$ & 0 & -2 & -1 \\
\hline
\end{tabular}




\begin{tabular}{|c|c|c|c|c|}
\hline \multirow[t]{2}{*}{ Nr. } & \multirow[t]{2}{*}{ Statement } & \multicolumn{3}{|c|}{ Faktoren } \\
\hline & & A & B & C \\
\hline $\mathrm{G} 37^{* *}$ & $\begin{array}{l}\text { Social Freezing hat den Vorteil, dass die Kinder älterer Mütter } \\
\text { noch jung und fit sind, wenn sie ihre pflegebedürftigen Eltern un- } \\
\text { terstützen müssen. }\end{array}$ & -2 & -2 & -1 \\
\hline G38 & $\begin{array}{l}\text { Social Freezing führt dazu, dass die Kinder ihre Grosseltern nur } \\
\text { noch im Pflegeheim oder gar nicht mehr erleben. }\end{array}$ & 1 & 2 & $-2^{*}$ \\
\hline \multicolumn{5}{|c|}{ Finanzielle Dimension } \\
\hline F39 & $\begin{array}{l}\text { Social Freezing entspricht eigentlich nicht dem Bedürfnis der Nut- } \\
\text { zerinnen. Die Nachfrage wird durch die Anbieter dieser Technolo- } \\
\text { gie (z.B. Reproduktionskliniken) künstlich gesteigert. }\end{array}$ & $1^{*}$ & $-1^{*}$ & $-4^{*}$ \\
\hline F40 & $\begin{array}{l}\text { Frauen müssen nicht vor den ökonomischen Interessen von An- } \\
\text { bietern des Social Freezing (z.B. Reproduktionskliniken) ge- } \\
\text { schützt werden. Das ist bevormundend und sexistisch. }\end{array}$ & -2 & -3 & $3^{*}$ \\
\hline F41 & $\begin{array}{l}\text { Unternehmen, die sich an den Kosten des Social Freezing beteili- } \\
\text { gen, setzen junge Frauen unter Druck, die Familiengründung zu- } \\
\text { gunsten ihrer Karriere zurückzustellen. }\end{array}$ & 1 & 3 & $-2^{*}$ \\
\hline F42 & $\begin{array}{l}\text { Ein Teil der Kosten für die künstliche Befruchtung sollte durch die } \\
\text { Krankenkassen übernommen werden. Sonst können sich nur gut } \\
\text { verdienende Frauen diese Technik leisten, was die soziale Un- } \\
\text { gleichheit fördert. }\end{array}$ & $-5^{*}$ & -1 & $4^{*}$ \\
\hline $\mathrm{F} 43^{* *}$ & $\begin{array}{l}\text { Kosten für Social Freezing sollen durch die Frauen selbst begli- } \\
\text { chen werden, weil ungewiss ist, ob die Frauen die eingefrorenen } \\
\text { Eizellen überhaupt je brauchen werden. }\end{array}$ & 1 & -1 & 1 \\
\hline F44 & $\begin{array}{l}\text { Kosten für Social Freezing sollen nicht von den Krankenkassen } \\
\text { übernommen werden, da der Eingriff nicht medizinisch notwendig } \\
\text { ist. }\end{array}$ & $2^{*}$ & 0 & 0 \\
\hline F45 & $\begin{array}{l}\text { Social Freezing bedeutet mehr Komplikationen bei älteren Frauen } \\
\text { aufgrund von Risikoschwangerschaften und Frühgeburten. Höhe- } \\
\text { re Kosten für die Allgemeinheit sind deshalb vorprogrammiert. }\end{array}$ & 2 & 1 & $-2^{*}$ \\
\hline F46 & $\begin{array}{l}\text { Dank Social Freezing gibt es mehr Kinder von Paaren, die sonst } \\
\text { kinderlos bleiben würden. Dies wirkt ein Stück weit den negativen } \\
\text { volkswirtschaftlichen Auswirkungen des demografischen Wandels } \\
\text { entgegen. }\end{array}$ & -1 & -3 & $1^{*}$ \\
\hline
\end{tabular}




\begin{tabular}{|c|c|c|c|c|}
\hline Nr. & Statement & Fakt & $n$ & C \\
\hline \multicolumn{5}{|c|}{ Rechtliche Dimension } \\
\hline R47 & $\begin{array}{l}\text { Die Aufbewahrungsfrist der eingefrorenen Eizellen sollte verlän- } \\
\text { gert werden (heute maximal zehn Jahre). Eine } 30 \text {-jährige Frau, } \\
\text { die Social Freezing macht, sollte die Eizellen auch über } 40 \text { noch } \\
\text { künstlich befruchten lassen dürfen. }\end{array}$ & $-3^{*}$ & 2 & 3 \\
\hline R48 & $\begin{array}{l}\text { Sinnvoller als eine Limitierung der Aufbewahrungsfrist für die } \\
\text { eingefrorenen Eizellen, wie sie in der Schweiz besteht, wäre eine } \\
\text { Altersgrenze der Frauen für die künstliche Befruchtung der Eizel- } \\
\text { len. }\end{array}$ & 0 & -2 & 1 \\
\hline R49 & $\begin{array}{l}\text { Auch alleinstehende Mütter sollten Social Freezing machen dür- } \\
\text { fen. Die rechtlichen Rahmenbedingungen der Fortpflanzungs- } \\
\text { medizin sind in der Schweiz diesbezüglich zu restriktiv. }\end{array}$ & $-1^{*}$ & 2 & 2 \\
\hline R50 & $\begin{array}{l}\text { Auch Frauen in homosexuellen Partnerschaften sollten Social } \\
\text { Freezing machen dürfen. Die rechtlichen Rahmenbedingungen } \\
\text { der Fortpflanzungsmedizin sind in der Schweiz diesbezüglich zu } \\
\text { restriktiv. }\end{array}$ & $-1^{*}$ & *4 & $1^{*}$ \\
\hline R51 & $\begin{array}{l}\text { Die rechtlichen Rahmenbedingungen der Fortpflanzungsmedizin } \\
\text { sind in der Schweiz zu restriktiv. Dies fördert den Fruchtbarkeits- } \\
\text { tourismus. }\end{array}$ & $-5^{*}$ & 1 & 0 \\
\hline
\end{tabular}

Legende: ${ }^{*}=$ «distinguishing factor» des jeweiligen Faktors und ${ }^{* *}=$ «consensus factor» für alle Faktoren (mit Signifikanzniveau von $\mathrm{P}<0.01$ ). 


\section{A4 Anhang: Rechtsvergleichung}

Im Anhang 4 werden die Erlasse, Materialien und die Literaturauswahl zur Rechtsvergleichung, gegliedert nach den sieben untersuchten Ländern, aufgeführt.

\section{A4.1 Österreich}

Relevante Erlasse

Bundesgesetz, mit dem Regelungen über die medizinisch unterstützte Fortpflanzung getroffen (Fortpflanzungsmedizingesetz - öFMedG) sowie das allgemeine bürgerliche Gesetzbuch, das Ehegesetz und die Jurisdiktionsnorm geändert werden, BGBI. Nr. 275/1992

Weiterführende Literatur (Auswahl)

Barth Peter, Die Entnahme von Zellen für Zwecke medizinisch unterstützter Fortpflanzung, in: Handbuch des neuen Fortpflanzungsmedizinrechts, Wien 2015, S. $199 \mathrm{ff}$.

Bernat Erwin, Einführung in das österreichische Medizinrecht, in: Handbuch des Fachanwalts Medizinrecht, 3. Aufl., Köln 2013, S. 1861 ff.

Derselbe, Das Recht der Fortpflanzungsmedizin im Wandel, Eckpunkte des Fortpflanzungsmedizinrechts-Änderungsgesetzes 2015, in: JAP 201 5/2016, S. $45 \mathrm{ff}$.

Czech Philip, Zur Grundrechtskonformität des neuen Fortpflanzungsmedizinrechts, in: NLMR 2015, S. $297 \mathrm{ff}$.

Egarter Christian, State of the Art in der Fortpflanzungsmedizin: Das Fortpflanzungsmedizinrechts-Änderungsgesetz 2015 aus der Sicht des Reproduktionsmediziners, in: Das Recht der Fortpflanzungsmedizin 2015 Analyse und Kritik, Wien 2016, S. $1 \mathrm{ff}$.

Erlebach Martina, Die Samen- und Eizellspende im FMedG, in: Handbuch des neuen Fortpflanzungsmedizinrechts, Wien 2015, S. 213 ff. 
Ferrari Susanne, Aktuelle Entwicklungen im österreichischen Familien- und Erbrecht, in: FamRZ 2015, S. 1556 ff.

Dieselbe, Künstliche Fortpflanzung im österreichischen Recht, in: Künstliche Fortpflanzung und europäisches Familienrecht, Bielefeld 2015, S. $181 \mathrm{ff}$.

Flatscher-Thöni Magdalena/Voithofer Caroline/Werner-Felmayer Gabriele, Fortpflanzungsmedizingesetz 2015: Lang ersehnte Liberalisierung ohne erwünschte Nebenwirkungen?, in: juridikum 2015, S. 26 ff.

Kopetzki Christian, «Social Egg Freezing», in: RdM 2014, S. 309

Derselbe, Das Recht der Fortpflanzungsmedizin 2015: Aktueller Stand und verfassungsrechtliche Bewertung, in: Das Recht der Fortpflanzungsmedizin 2015 - Analyse und Kritik, Wien 2016, S. 63 ff.

Neumayr Matthias/Resch Reinhard/Wallner Felix (Hrsg.), Gmundner Kommentar zum Gesundheitsrecht (GmundKomm), Wien 2016

Swoboda Martin/Loimer Leonhard, Die «Assistierte Reproduktionstechnik» aus medizinischer Sicht, in: Handbuch des neuen Fortpflanzungsmedizinrechts, Wien 2015, S. $37 \mathrm{ff}$.

Wendehorst Christiane, Neuerungen im österreichischen Fortpflanzungsmedizinrecht durch das FMedRÄG 2015, Anpassung an europaweite Entwicklungen, in: iFamZ 2015, S. 4 ff.

Zwettler Adrian, Durchbruch oder Zwischenschritt?, Eine Analyse der Neuerungen im FMedG unter Berücksichtigung weiterhin offener Aspekte, in: ZfG 2016, Heft 2, S. 48 ff.

\section{Materialien}

Bioethikkommission, Stellungnahme der Bioethikkommission beim Bundeskanzleramt zum Entwurf eines Bundesgesetzes, mit dem das Fortpflanzungsmedizingesetz, das Allgemeine bürgerliche Gesetzbuch und das Gentechnikgesetz geändert werden (Fortpflanzungsmedizinrechts-Änderungsgesetz 2015 - FMedRÄG 2015) vom 28. November 2014 


\section{A4.2 Frankreich}

\section{Relevante Erlasse}

Code de la santé publique

Loi $n^{\circ}$ 2011-814 du 7 juillet 2011 relative à la bioéthique (NOR: ETSX1117652L) Arrêté du 30 juin 2017 modifiant l'arrêté du 11 avril 2008 modifié relatif aux règles de bonnes pratiques cliniques et biologiques d'assistance médicale à la procréation (NOR: SSAP1719338A)

Décret $n^{\circ}$ 2015-1281 du 13 octobre 2015 relatif au don de gamètes (NOR: AFSP1513121D)

Arrêté du 24 décembre 2015 pris en application de l'article L. 2141-1 du code de la santé publique et modifiant l'arrêté du 3 août 2010 modifiant l'arrêté du 11 avril 2008 relatif aux règles de bonnes pratiques cliniques et biologiques d'assistance médicale à la procréation (NOR: AFSP1532457A)

\section{Weiterführende Literatur (Auswahl)}

Académie nationale de Médecine, La conservation des ovocytes, Rapport vom 13. Juni 2017, Paris 2017 <http://www.academie-medecine.fr/wpcontent/uploads/2017/06/La-conservation-des-ovocytes-version-15juin-2017.pdf> (besucht am: 13. Mai 2018)

Belaisch-Allart Joëlle et al., Réflexions sur les indications sociétales de la préservation de la fertilité féminine, in: MT Médecine de la Reproduction, Gynécologie Endocrinologie 2013, S. $111 \mathrm{ff}$.

Bellivier Florence/Hennette-Vauchez Stéphanie, Bioéthique, in: Cahiers Droit, Sciences \& Technologies 2016, Heft 6, Rz. 1 ff. <http://cdst.revues. org/513> (besucht am: 13. Mai 2018)

Binet Jean-René, Décret $n^{\circ} 2015-1281$ du 13 octobre 2015 ou comment inciter au don d'ovocytes sans le dire, in: Droit de la famille $n^{\circ} 1$, Januar 2016, Comm. n¹7

Derselbe, Quel âge pour accéder à l'assistance médicale à la procréation?, in: Droit de la famille ${ }^{\circ} 5$, Mai 2017, Comm. ${ }^{\circ} 112$ 
Derselbe, Demandes sociétales de recours à l'assistance médicale à la procréation : vers la grande rupture?, in: Droit de la famille $n^{\circ} 9$, September 2017, Comm. $n^{\circ} 9$

Chaltiel Florence, Donner la vie, de quel(s) droit(s)?, Nouvelles réflexions après l'avis du comité consultatif national d'éthique, in: Petites affiches, 19.20. September 2017, n¹87-188, S. 7 ff.

Depadt-Sebag Valérie, Droit et bioéthique, 2. Aufl., Brüssel 2012

Lambert-Garrel Lucile, Ouverture du don de gamètes aux personnes sans enfants, in: Revue droit \& santé $n^{\circ} 69$, Januar 2016, S. 76 f.

Dieselbe, Les nouvelles règles de bonnes pratiques cliniques et biologiques d'assistance médicale à la procréation, in: Revue droit \& santé $n^{\circ} 70$, März 2016, S. 235 f.

Dieselbe, L'âge paternel peut-il être un obstacle à l'accès à l'assistance médicale à la procréation?, in: Revue droit \& santé $n^{\circ} 77$, Mai 2017 , S. $375 \mathrm{ff}$.

Legros Bérengère, Droit de la bioéthique, Bordeaux 2013

Penneau Jean, Corps humain - Bioéthique, in: Répertoire de droit civil, Paris 2012 (aktualisiert April 2017)

Pintens Walter, Künstliche Fortpflanzung im belgischen und französischen Recht, in: Künstliche Fortpflanzung und europäisches Familienrecht, Bielefeld 2015, S. $105 \mathrm{ff}$.

Vialla François, Assistance médicale à la procreation, avis du CCNE, in: Recueil Dalloz 2017, S. 1365 f.

Materialien

Comité Consultatif National d'Ethique, Avis n¹26 (15 juin 2017), Avis du CCNE sur les demandes sociétales de recours à l'assistance médicale à la procréation (AMP) 


\section{A4.3 Italien}

Relevante Erlasse

Legge 19 febbraio 2004, n. 40 - Norme in materia di procreazione medicalmente assistita (pubblicata nella Gazzetta Ufficiale n. 45 del 24 febbraio 2004)

Linee guida contenenti le indicazioni delle procedure e delle tecniche di procreazione medicalmente assistita (pubblicata nella Gazzetta Ufficiale n. 161 del 14 luglio 2015)

Regolamento recante norme in materia di manifestazione della volontà di accedere alle tecniche di procreazione medicalmente assistita, in attuazione dell'articolo 6, comma 3, della legge 19 febbraio 2004, n. 40 (pubblicata nella Gazzetta Ufficiale n. 40 del 17 febbraio 2017)

Weiterführende Literatur (Auswahl)

Balistreri Maurizio/Pollo Simone, Storia della normativa sulla procreazione assistita in Italia, in: Bioetica 3/2005, S. 19 ff.

Ciraolo Clorinda, Certezza e stabilità delle relazioni familiari nella procreazione medicalmente assistita, in: Ordine internazionale e diritti umani 2016, S. $812 \mathrm{ff}$.

Göllner Eva, Aktuelle strafrechtliche Fragestellungen zur Präimplantationsdiagnostik, Eine Darstellung der deutschen Rechtslage vor dem Hintergrund möglicher Alternativverfahren und unter besonderer Berücksichtigung der Rechtslage in Italien, Berlin 2008

Zaccaria Alessio (Hrsg.), Commentario breve al diritto della famiglia, 3. Aufl., Vicenza 2016 


\section{A4.4 Deutschland}

\section{Relevante Erlasse}

Gesetz zum Schutz von Embryonen (Embryonenschutzgesetz - ESchG), BGBI.

I S. 2746

Gesetz über die Spende, Entnahme und Übertragung von Organen und Geweben (Transplantationsgesetz - TPG), BGBI. I S. 2206

Verordnung über die Anforderungen an Qualität und Sicherheit der Entnahme von Geweben und deren Übertragung nach dem Transplantationsgesetz (TPG-Gewebeverordnung - TPG-GewV), BGBI. I S. 512

Gesetz über den Verkehr mit Arzneimitteln (Arzneimittelgesetz - AMG), BGBI. I S. 3394

Verordnung über die Anwendung der Guten Herstellungspraxis bei der Herstellung von Arzneimitteln und Wirkstoffen und über die Anwendung der Guten fachlichen Praxis bei der Herstellung von Produkten menschlicher Herkunft (Arzneimittel- und Wirkstoffherstellungsverordnung AMWHV), BGBI. I S. 2523

(Muster-)Richtlinie zur Durchführung der assistierten Reproduktion (Novelle 2006; diese Richtlinie, die als blosses Standesrecht keine formelle Rechtskraft entfaltet, wird voraussichtlich durch die «Richtlinie zur Entnahme und Übertragung von menschlichen Keimzellen im Rahmen der assistierten Reproduktion» ersetzt)

Weiterführende Literatur (Auswahl)

Günther Hans-Ludwig/Taupitz Jochen/Kaiser Peter, Embryonenschutzgesetz, Juristischer Kommentar mit medizinisch-naturwissenschaftlichen Grundlagen, 2. Aufl., Stuttgart 2014

Höfling Wolfram (Hrsg.), TPG - Transplantationsgesetz, Kommentar, 2. Aufl., Berlin 2013

Hübner Marlis/Pühler Wiebke, Systematische Rechtsentwicklung in der Reproduktionsmedizin, in: MedR 2017, S. $929 \mathrm{ff}$.

Joecks Wolfgang/Miebach Klaus (Hrsg.), Münchener Kommentar zum Strafgesetzbuch, Band 6: JGG (Auszug), Nebenstrafrecht I, 2. Aufl., München 2013 (Kommentierung TPG) 
Jofer Patricia, Regulierung der Reproduktionsmedizin, Fremdsamenspende, Ersatzmutterschaft und Umgang mit überzähligen Embryonen, BadenBaden 2014

Jungfleisch Frank, Fortpflanzungsmedizin als Gegenstand des Strafrechts, Eine Untersuchung verschiedenartiger Regelungsansätze aus rechtsvergleichender und rechtspolitischer Perspektive, Berlin 2005

Keller Rolf/Günther Hans-Ludwig/Kaiser Peter, Embryonenschutzgesetz, Kommentar zum Embryonenschutzgesetz, Stuttgart 1992

Kügel Wilfried/Müller Rolf-Georg/Hofmann Hans-Peter (Hrsg.), Arzneimittelgesetz, Kommentar, 2. Aufl., München 2016

Möller Karl-Heinz/Hilland Ulrich, Kryokonservierung von Keimzellen - Rechtlicher Rahmen und Vertragsgestaltung, in: Reproduktionsmedizin, Rechtliche Fragestellungen, Dokumentation der Tagung zum 10-jährigen Bestehen des Instituts für Rechtsfragen der Medizin Düsseldorf, Düsseldorf 2010, S. $125 \mathrm{ff}$.

Müller-Terptiz Ralf/Ruf Isabelle, Die «medizinisch unterstützte Befruchtung» als Gegenstand des Arzneimittel- und Transplantationsrechts, in: Aktuelle Herausforderungen der Life Sciences, Berlin 2010, S. $33 \mathrm{ff}$.

Prütting Dorothea (Hrsg.), Medizinrecht Kommentar, 4. Aufl., Köln 2016

Spickhoff Andreas (Hrsg.), Medizinrecht, 2. Aufl., München 2014

Materialien

Enquete-Kommission «Recht und Ethik der modernen Medizin», Schlussbericht vom 14. Mai 2002, BT-Drs. 14/9020

Deutscher Bundestag, Gesetzesentwurf der Bundesregierung, Entwurf eines Gesetzes über Qualität und Sicherheit von menschlichen Geweben und Zellen (Gewebegesetz) vom 25. Oktober 2006, BT-Drs. 16/3146

\section{A4.5 Belgien}

Relevante Erlasse

Loi relative à la procréation médicalement assistée et à la destination des embryons surnuméraires et des gamètes du 6 juillet 2007 
Weiterführende Literatur (Auswahl)

Derèse Marie-Noëlle/Willems Geoffrey, La loi du 6 juillet 2007 relative à la procréation médicalement assistée et à la destination des embryons surnuméraires et des gamètes, in: Revue trimestrielle de droit familial 2008, S. $279 \mathrm{ff}$.

Genicot Gilles, La maîtrise du début de la vie : la loi du 6 juillet 2007 relative à la procréation médicalement assistée, in: Journal des tribunaux 2009, S. $17 \mathrm{ff}$.

Nys Hermann/Wuyts Tim, De wet betreffende de medisch begeleide voortplanting en de bestemming van de overtallige embryo's en de gameten, in: Rechtskundig Weekblad 2007/2008, S. 762 ff.

Pintens Walter, Künstliche Fortpflanzung im belgischen und französischen Recht, in: Künstliche Fortpflanzung und europäisches Familienrecht, Bielefeld 2015, S. $105 \mathrm{ff}$.

Schamps Geneviève, L'autonomie de la femme et les interventions biomédicales sur son corps en droit belge, in: Corps de la femme et Biomédecine, Brüssel 2013, S. $39 \mathrm{ff}$.

\section{Materialien}

Rapport fait au nom du Groupe de travail «bioéthique» par Mme Clotilde Nyssens, Doc. parl., Sénat de Belgique 2004-2005, Nr. 3-418/2

Proposition de loi relative à la procréation médicalement assistée et à la destination des embryons surnuméraires et des gamètes (Déposée par Mme Christine Defraigne, M. Patrik Vankrunkelsven, Mme Jacinta De Roeck, M. Philippe Mahoux et Mme Isabelle Durant), Doc. parl., Sénat de Belgique 2005-2006, Nr. 3-1440/1

Rapport fait au nom de la commission des affaires sociales par M. Jean Cornil et Mme Mia De Schamphelaere, Doc. parl., Sénat de Belgique 20052006, Nr. 3-1440/9 


\section{A4.6 Spanien und Tschechische Republik}

Zu Spanien wurde ein Rechtsgutachten von Prof. Josep Ferrer Riba, Universität Pompeu Fabra, Barcelona, eingeholt. Zur Tschechischen Republik wurde ein Gutachten von Dr. Lenka Westphalova, Juristische Fakultät Olomouc (Olmütz), Tschechien, eingeholt. Der Inhalt dieser Gutachten wurde vorne in redaktionell bearbeiteter Form wiedergegeben. 


\section{Mitglieder der Begleitgruppe}

Prof. Dr. Alberto Bondolfi (Präsident der Begleitgruppe), Universität Genf, TASWISS Leitungsausschuss

Ulrike Baureithel, freie Journalistin und Lehrbeauftragte an der HumboldtUniversität zu Berlin

Prof. Dr. Urs Brügger, Institutsleiter Winterthurer Institut für Gesundheitsökonomie, ZHAW School of Management and Law (bis Dezember 2017), Direktor Departement Gesundheit, Berner Fachhochschule (ab Januar 2018)

Dr. Nolwenn Bühler, STS Lab - Laboratoire d'études des sciences et des techniques, Institut des sciences sociales, Université de Lausanne \& Laboratoire d'études des processus sociaux, Facultés des Lettres et Sciences Humaines, Université de Neuchâtel

Dr. Matthias Bürgin, Juristische Fakultät der Universität Basel \& Bundesamt für Gesundheit (BAG)

Dr. Nadja Eggert, Ethos - Plateforme interdisciplinaire d'éthique de l'Unil, Université de Lausanne

Prof. Dr. med. Bruno Imthurn, Klinik für Reproduktions-Endokrinologie, UniversitätsSpital Zürich

Dr. Fridolin Marty, Leiter Gesundheitspolitik, economiesuisse

Prof. Dr. Frank Mathwig, Schweizerischer Evangelischer Kirchenbund, Mitglied NEK

Prof. Dr. med. Urs Scherrer, Universitätsklinik für Kardiologie, Inselspital Bern

Dr. Heidi Simoni, Leitung Marie Meierhofer Institut für das Kind (MMI)

Prof. Dr. med. Giatgen A. Spinas, Universitätsspital Zürich, TA-SWISS Leitungsausschuss 


\title{
Mitglieder der Projektgruppe
}

Prof. Dr. Andreas Balthasar, Interface Politikstudien Forschung Beratung \& Titularprofessor für Politikwissenschaften, Universität Luzern

Prof. Dr. Regina E. Aebi-Müller, Professorin für Privatrecht und Privatrechtsvergleichung, Universität Luzern

Prof. Dr. Christian Kind, Titularprofessor für Pädiatrie, Universität Zürich

Sarah Fässler, Interface Politikstudien Forschung Beratung

Franziska Müller, Interface Politikstudien Forschung Beratung

Vera Hertig, Interface Politikstudien Forschung Beratung

Alexander Lueger, Universität Luzern

\section{Projektmanagement TA-SWISS}

\author{
Dr. Elisabeth Ehrensperger, Geschäftsführerin \\ Dr. Christina Tobler, Projektleiterin
}




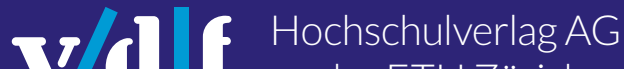 an der ETH Zürich}

\section{Liebe Leserin, lieber Leser}

Wir freuen uns, dass Sie unsere Open-Access-Publikation

heruntergeladen haben. Der vdf Hochschulverlag fördert Open Access aktiv und publiziert seit 2008 Gratis-eBooks in verschiedenen Fachbereichen:

\section{Übersicht Open-Access-Titel}

\section{Möchten auch Sie Open Access publizieren?}

Der vdf Hochschulverlag stellt Ihre Publikation u.a. im eigenen Webshop sowie der ETH-Research-Collection zum Download bereit!

Kontaktieren Sie uns unter verlag@vdf.ethz.ch

Gerne informieren wir Sie auch in Zukunft über unsere

(Open-Access-)Publikationen in Ihrem Fachbereich.

\section{Newsletter abonnieren}

Auch Sie können Open Access unterstützen.

Hier geht's zum Spenden-Button

Herzlichen Dank! 


\section{www.ta-swiss.ch}

Ein Kompetenzzentrum der

\section{+ akademien der wissenschaften schweiz}

Eigene Eizellen einfrieren und später, mit dem Wunschpartner oder im günstigen Moment, in der Glasschale befruchten lassen: Social Freezing verspricht, Frauen über den Zeitpunkt einer gewünschten Schwangerschaft selber entscheiden zu lassen, obne Druck durch die biologische Uhr. Doch das Verfahren wirft auch Fragen auf; nach medizinischen Risiken, dem Kindeswobl und der gesellschaftlichen Akzeptanz. Begünstigt Social Freezing die Vereinbarkeit von Beruf und Familie, oder fübrt es zur Vernachlässigung anderer Massnabmen, um Arbeitsleben und Familienplanung in Einklang zu bringen?

Die interdisziplinäre Studie von TA-SWISS wägt die Chancen und Risiken des Social Freezing ab. Sie untersucht, wie viele Frauen in der Schweiz am Social Freezing interessiert sind und schätzt ab, wie gross das Potenzial der Technologie in Zukunft sein könnte. Die Studie befragt Schweizer Akteure und zeigt die verschiedenen Meinungsbilder zu dem Thema auf. Weiter geht sie darauf ein, welche rechtlichen, ethischen, gesellschaftlichen und medizinischen Kriterien beachtet werden sollten. Damit ermöglicht sie einen vielseitigen Blick auf das Thema Social Freezing und liefert Grundlagen für die weiterführende Debatte.

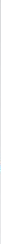

\section{v/llif}

TA-SWISS 69/2019

ISBN 978-3-7281-3962-7 (Printausgabe)

ISBN 978-3-7281-3963-4 (E-Book)

DOI-Nr. 10.3218/3963-4 